\title{
Office of Military \\ Government for Hesse
}

Von

Dieter Emig und Alfred G. Frei 



\section{A. Historischer Überblick}

\section{Vorgeschichte}

Nachdem General Dwight D. Eisenhower durch die Proklamation Nr. 2 vom 19. September 1945 die Gründung des Landes Groß-Hessen verkündet hatte, übernahm das Detachment E-5 der amerikanischen Militärregierung für Deutschland am 8. Oktober 1945 in Wiesbaden die Kontrolle über den gesamten Landesbereich. ${ }^{1}$

Dieses Detachment war am 15.September 1944 in Rochefort en Yveline (Frankreich) im Rahmen der European Civil Affairs Division aufgestellt worden. ${ }^{2}$ Es trug die Bezeichnung E1 A2 und war ursprünglich als Regionaldetachment für den Bereich SaarPfalz-Rheinhessen vorgesehen. Sowohl während des Aufenthalts in Frankreich als auch beim Vormarsch war der Kommandierende Offizier von E1 A2 gleichzeitig für alle anderen Detachments, die in seinem regionalen Bereich arbeiten sollten, verantwortlich.

Vor Erreichen der "statischen Phase“ - so bezeichneten die Amerikaner die Zeit nach dem Sieg über die Armeen der Nationalsozialisten - waren jedoch die meisten dieser Detachments taktischen Truppen zugeordnet. ${ }^{3}$ Eindrucksvoll schildert der erste Tagesbericht des Detachments diese Situation:

„Die Rolle, die das Regional Detachment während der Kampfphase spielte, unterschied sich [... .] sehr von dem, was sich viele Military Government Officers vorgestellt hatten. Mangelndes Verständnis für die Aufgabenstellung dieser Art von Detachment und das Fehlen einer klaren Direktive von höherer Ebene waren zwei Faktoren, die echte Hindemisse für eine erfolgreiche Organisation der Militärregierung darstellten und zu Verwirrung, Frustration und Unzufriedenheit beitrugen, die sich wie anstekkende Krankheiten unter den Mitarbeitern des Detachments immer weiter verbreiteten. Trotz der langen Wartezeit und der ermuldenden Unterrichtsstunden, die Offiziere und Mannschaften zuvor hinter sich gebracht hatten, waren die Erwartungen in den Wochen nach Aufstellung des Detachments groB. Aber die unzureichende Klärung der Aufgaben und Tätigkeiten des Teams [...] bedrohte ernsthaft seine Moral und Leistungsfähigkeit.

Der tagtägliche Ärger, fehlende eigene Unterkünfte, unzureichende Verpflegungsund Versorgungseinrichtungen, der ergebnislose und sich ständig wiederholende Ausbildungsplan, das moralische und geistige ,Abschlaffen' von Offizieren und Soldaten, die nichts anderes zu tun hatten, als sich mit ihrem persönlichen Wohlergehen und ihren eigenen Launen zu beschäftigen - all dies trug im Laufe der Zeit viel dazu bei, den Zusammenhalt und den Enthusiasmus zu zerstören, der die meisten Mitarbeiter des Detachments in den ersten Wochen ihrer Arbeit beseelt hatte. ${ }^{.4}$

${ }^{1}$ Chronology Military Government Detachment E1A2 (E-5) and Office of Military Government for Hesse 1944 - 1947 (Staatsarchiv Darmstadt), S. 5 (zit: Chronology).

${ }^{2}$ Ebenda, S. 1.

${ }^{3} 8 / 187-1 / 2$.

${ }^{4}$ Ebenda. 
Ubersicht 1: Organisation und Offiziere des Detachments E1A2 bei seiner Aufstellung in Rochefort en Yveline beim Hauptquartier der European Civil Affairs Division (ECAD), 15.September 1944

Tätigkeitsbereich

Military Government Officer

Deputy Mil.Gov. Officer

Executive Officer

Assistant Exec. Officer

Administrative Officer

Fire Section

Econ. and Public Utilities

Econ. and Public Utilities

Econ. and Public Utilities

Property Control

Property Control

Property Control

Post, Telephone, Telegraph

Labor

Displaced Persons

Displaced Persons

Displaced Persons

Displaced Persons

Food

Supply

Legal

Legal

Legal

Administration and Legal

Publ. Monuments and Fine Arts

Public Safety

Public Safety

Public Safety

Education and Relig. Affairs

Histor.and Polit.Information

Transport

$\begin{array}{ll}\text { Dienstgrad } & \text { Name } \\ \text { Lt.Col. } & \text { Donat M. Wilson } \\ \text { Lt.Col. } & \text { Norman E. Routzohn } \\ \text { Major } & \text { Irwin Harlow } \\ \text { Major } & \text { Myron I. Barker } \\ \text { 1st Lt. } & \text { Robert Wallach } \\ \text { Capt. } & \text { James L. Turner } \\ \text { Major } & \text { Albert J. Gottlieb } \\ \text { Capt. } & \text { James W. Nicol } \\ \text { 2nd Lt. } & \text { George E. Roese } \\ \text { Major } & \text { Myron M. Maupin } \\ \text { Major } & \text { John D. Picco } \\ \text { 1st Lt. } & \text { Francis R. Paternoster } \\ \text { Major } & \text { Dalton D. Irwin } \\ \text { Capt. } & \text { Glenn E. Garrett } \\ \text { Capt. } & \text { Archibald R. Giroux } \\ \text { 2nd Lt. } & \text { Hillard A. Rhoades } \\ \text { 1st Lt. } & \text { Rex M. Smith } \\ \text { 1st Lt. } & \text { Harold A. Wyatt } \\ \text { Capt. } & \text { Thomas B. Keith } \\ \text { Capt. } & \text { William G. Mueller } \\ \text { Lt.Col. } & \text { Frank Seydel } \\ \text { Capt. } & \text { Franklin J. Potter } \\ \text { 1st Lt. } & \text { Charles H. Story } \\ \text { Major } & \text { Charles P. Sisson } \\ \text { Capt. } & \text { Harold W. Landin } \\ \text { Capt. } & \text { John F. Mumane } \\ \text { Major } & \text { August F. Berendt } \\ \text { 1st Lt. } & \text { Thomas W. Harris } \\ \text { Major } & \text { James F. Bursch } \\ \text { 1st Lt. } & \text { Edgar L. Erickson } \\ \text { 1st Lt. } & \text { Charles H. Hennessy } \\ & \end{array}$

Kommandierender Offizier des Detachments E1A2 war Lt.Col. Donat M. Wilson. Als Stellvertreter fungierte bis zum 25. September 1944 Lt.Col. Frank Seydel, später dann Lt.Col. Norman E. Routzohn. Am 28. Oktober 1944 ubernahm Lt.Col. Eugene Bond dieses Amt. Routzohn wurde zum Property Control Officer bestimmt. Diese Ablösung stand vermutlich in Zusammenhang mit den großen Disziplinproblemen, mit denen E1A2 und die untergeordneten Detachments in jener Zeit zu kämpfen hatten.

So waren bereits am 20. Oktober 1944 durch eine Razzia Unregelmäßigkeiten in der Messe der Company A festgestellt worden. Daraufhin wurde Capt. Hudson als kommandierender Offizier abgelöst und Lt. Peterson zu seinem Nachfolger emannt.

Eines der wichtigsten Probleme während der in Etain fortgesetzten Ausbildung war die Frage der zukünftigen Behandlung der Displaced Persons (DPs). Während die Unterrichtung der Detachment Officers durch Spezialisten von E1A2 ihren Fortgang nahm, blieb diese Frage weithin ungeklärt. Trotzdem wurden die Detachments nach

${ }^{5}$ Scheidet am 24. September 1944 aus. 
dem 20. November - sie waren inzwischen über Joudreville bis Maxeville bei Nancy vorgerückt - zunehmend für Aufgaben im DP-Bereich herangezogen. Am 25. November 1944 vermeldete der Chronist von E1A2 das Ende des offiziellen Ausbildungsprogramms.

Die Stärke des Regional Detachment E1 A2 betrug nach verschiedenen Zugängen am 17. Dezember 1944118 Offiziere und 173 Soldaten. Von den Offizieren waren zu diesem Zeitpunkt 51 in Maxeville stationiert, 67 befanden sich im Feld. ${ }^{6}$ Nach einer Zwischenstation in Esch (Luxemburg) erreichte das Detachment E1 A2 am 28. April 1945 Neustadt in der Pfalz.

Kurz zuvor, am 26. April 1945, hatte Colonel James R. Newman, geboren 1903, das Kommando von E1A2 übertragen bekommen. Im Zivilberuf an führender Stelle in einer Schulaufsichtsbehörde tätig und promovierter Erziehungswissenschaftler, konnte er auf eine erfolgreiche militärische Karriere zurückblicken. Als Absolvent der Militärakademie in West Point hatte er sich bereits 1934 freiwillig gemeldet und war schließlich 1941 in den aktiven Dienst berufen worden. Seine militärischen Sporen verdiente er sich als kommandierender Offizier in verschiedenen Einheiten der Küstenwache und der Luftabwehr. Später wurde er aufgrund seiner beruflichen Erfahrung zum ersten Militärregierungs-Regiment versetzt und brachte es nach einer Zwischenstation in England bis zum kommandierenden Offizier in der Vorhut der European Civil Affairs Division in Frankreich. Von dort wurde er 1944 nach England zurückberufen, um die Organisation der Militärregierung für Württemberg zu übernehmen. Aus welchen Gründen schließlich das Kommando von E1 A2 an Col. James R. Newman übergeben worden war, konnte nicht geklärt werden. ${ }^{7}$ Jedenfalls blieb es ihm vorbehalten, am 18. Mai 1945 die erste deutsche Zivilregierung nach Kriegsende in ihr Amt einzuführen. An ihrer Spitze stand Hermann Heimerich. Weitere Mitglieder der nach amerikanischen Angaben quasi handverlesenen Regierung waren Dr. Wilhelm Zutt, Dr. Hans Anschultz, Dr. Alexander Mitscherlich, Adolf Rausch und Emil Henk. ${ }^{8}$ Diese Regierung war zuständig für die späteren Bezirke Trier, Koblenz, Saarland (Saarbrücken), Pfalz (Neustadt) und Rheinhessen (Mainz). ${ }^{9}$

$\mathrm{Da}$ die linksrheinischen Gebiete von den Franzosen beansprucht wurden, bereitete sich das Detachment in den ersten Julitagen 1945 auf den Umzug von Neustadt nach Wiesbaden vor. In Besprechungen mit Vertretern der französischen Militärregierung (Col. Bonne, Maj.Gen. Bouley) wurde die Übergabe des Gebiets vorbereitet, die am 10. Juli 1945 feierlich vollzogen wurde. Im Tagesbericht heißt es, daß „die meisten Deutschen den Abzug der Amerikaner nicht gern sahen“. ${ }^{10}$

\section{Vom Regional Detachment zur Landesmilitärregierung}

Am 16. Juli 1945 übernahm Detachment E1 A2 die offizielle Befehlsgewalt über den Regierungsbezirk Wiesbaden und bezog sein Hauptquartier im Landeshaus. Capt. Landin, der zuständige Offizier für die Zivilverwaltung, reiste sogleich nach Mann-

\footnotetext{
$6 / 187-1 / 2$.

7 Weitere biographische Angaben zu James R. Newman in 8/216-3/3, 8/217-1/3 und Dorn: Inspektionsreisen in der US-Zone.

${ }^{8}$ Kurzbiographien der Regierungsmitglieder, der Text der Antrittsrede von H. Heimerich und eine Beschreibung des Festaktes in 8/217-1/3.

9 8/187-1/1.

10 Ebenda.
} 
heim, Heidelberg und Konstanz, um geeignete Mitglieder für eine deutsche Zivilregierung zu suchen.

Die ersten Tage in Wiesbaden waren bestimmt durch Kompetenzabgrenzungen gegenüber dem für die Stadt Wiesbaden zuständigen Detachment F1D2, ersten „Säuberungsmaßnahmen" in deutschen Behörden sowie Informationsbeschaffung über den Regierungsbezirk. Im Mittelpunkt standen weiterhin Ernährungs-, Gesundheits-, Sicherheits- und DP-Probleme.

Am 26. Juli 1945 legte das Hauptquartier von E1 A2 mit Organizational Directive Nr. 2 die Aufgaben und Verantwortlichkeiten des Regierungspräsidiums fest. Die personelle Besetzung regelten die Personnel Directions Nr.1-5."1 Organizational Directive Nr. 3 vom selben Tage sprach dem Regierungspräsidenten zum 1. August die Zuständigkeit für folgende Bereiche zu - selbstverständlich unter der Kontrolle der Militärregierung:

- Landwirtschaft, Gewässer und Forsten,

- Sozial- und Gesundheitswesen,

- Post,

- Erziehung und Kultus,

- Arbeit und Sozialversicherung und

- Wohnungswesen.

Auf einem Treffen der stellvertretenden Kommandeure von Detachment E1A2, Lt.Col. Howard R. Cress und von E1D2 (ehemals SHAEF Area Frankfurt), Lt.Col. Stone, am 31. Juli 1945, an dem auch die Wirtschaftsfachleute der beiden Detachments teilnahmen, einigte man sich auf folgende Punkte:

„Der Wirtschaftsabteilung (Department of Economics) des Regierungsbezirks Wiesbaden obliegt die zivile Überwachung der Industrie- und Handelskammern, der Handwerkskammern, des Landwirtschaftsamts und der Preisbildungs- und Preiskontrollbehörden.

Unter Aufsicht des Wiesbadener Regierungspräsidiums standen früher der Stadtkreis Frankfurt sowie die Landkreise Main-Taunus, Offenbach, Obertaunus, Friedberg und Hanau. Sie gehörten damals wie heute in die gewachsene Wirtschaftsstruktur des Regierungsbezirks Wiesbaden und sollten deshalb auch weiterhin im Zuständigkeitsbereich dieses Regierungsbezirks bleiben. Die Offiziere von Detachment E1A2 stimmen der Empfehlung zu, daB dieser BeschluB - soweit sie davon betroffen sind - angenommen wird. Die endgültige Entscheidung obliegt der Abteilung G-5 (Stabsabteilung: Civil Affairs - Military Government Section) bei der 7. Armee." 12

Diese Beschlüsse sind vor dem Hintergrund der Auflösung von SHAEF in der zweiten Juli-Hälfte zu sehen. Damit hatte auch die „curious creation“ SHAEF Enclave Frankfurt ihr Ende gefunden. ${ }^{13}$ Weitere wichtige Aufgaben, denen sich das Detachment be-

11 8/216-3/5. Dort auch das Operational Memorandum Nr. 1 und 2 vom 8. und 10. August 1945, die das Verhältnis zwischen Regierungsbezirk und Militärregierung regelten.

12 OMGHE: Historical Report July 1945.

${ }^{13}$ Siehe die von USFET Headquarters am 27. Juli 1945 erlassene „Directive on Reassignment of Responsibilities of the USFET Restricted Area ${ }^{\text {( }}(8 / 90-1 / 9)$ und Organizational Direction Nr. 11, Det. E-5, 8.10.1945 (5/41-1/20). Harold Zink, Professor für Politische Wissenschaft und selbst an prominenter Stelle in der Militärverwaltung tätig, hatte die SHAEF Enklave schon 1947 so kommentiert: „The American commandant of SHAEF at Frankfurt decided that it would be very nice if he could have a little feudal state to play with" (Zink: Military Government, S.97). 
reits im Juli widmete, waren das Transport- und Erziehungswesen sowie der städtische Wiederaufbau. Unklarheiten bestanden im Bereich der Property Control (Vermögenskontrolle). ${ }^{14}$

Mitte August wurden die ursprünglichen Detachment-Bezeichnungen, die Größe und Truppenzugehörigkeit angegeben hatten, geändert. E1A2 wurde nun E-5. ${ }^{15} \mathrm{Im} \mathrm{Au-}$ gust beschäftigte sich E-5 schwerpunktmäBig mit der Reorganisierung und Zentralisierung der deutschen Zivilverwaltung. Dazu ist dem Historical Report vom August 1945 zu entnehmen, daß die Militärregierung im „Kindergarten-Stadium“ ansetzen mußte, was die deutschen Fähigkeiten $2 u$ einer eigenständigen Verwaltung betraf. Weiter heißt es: „Preußische Bürokratie und Organisationspläne der Militärregierung waren nicht miteinander vereinbar“. Wenig optimistisch schlieBt der Report: „das Verrücken eines Schreibtisches brachte Kalamitäten mit sich und der Umzug eines Büros war eine Katastrophe".

Die Verwaltung unter der Leitung des Regierungspräsidenten wurde mit der Organizational Directive Nr. 10 vom 31. August 1945 in zehn Abteilungen gegliedert. ${ }^{16} 600$ Bedienstete des Regierungspräsidiums waren wegen Nazi-Aktivitäten entlassen worden. Die Militärregierung setzte ein Ausbildungsprogramm für die einzelnen Abteilungen in Gang, auch um die von den Entlassungen bedingten Lucken zu schließen. Über die Arbeit der lokalen beratenden Ausschulsse (Advisory Councils) äuBert sich der Bericht unzufrieden. Die Mitglieder dieser Ausschüsse hätten ihre Positionen oft dazu benutzt, sich und ihren Bekannten persönliche Vorteile zu verschaffen.

Den lokalen Militärregierungen und deutschen Verwaltungseinheiten sollte die Notwendigkeit einer zentralen Kontrolle uber verschiedene Wirtschaftsbereiche vermittelt werden. Der Regierungspräsident war ermächtigt, untergeordneten Stellen Anweisungen zu geben. Verwaltungsvorschriften muBten allerdings vor ihrer Veröffentlichung zweisprachig der Militärregierung vorgelegt werden. In dringenden Fällen konnten auch die örtlichen Militarregierungen Anordnungen des Regierungspräsidenten aufheben oder beim Chef der Militărregierung des Regierungsbezirks, dem Senior Military Government Officer, verlangen, daß dieser den Regierungsprăsidenten zur Änderung seiner Anweisungen auffordert. Ein Vetorecht hatten sie jedoch nicht.

Eines der schwierigsten Probleme der Militärregierung war es, die Machtbefugnisse des Regierungspräsidenten gegenuber den Landräten und Oberbürgermeistern durchzusetzen, ohne dabei in Konflikt mit der Verantwortlichkeit der Kreis-Militärregierungen zu geraten, die jene in ihre Ämter eingesetzt hatten. ${ }^{17}$ Beide Instanzen hatten sich vor Ort inzwischen weitgehend aneinander gewöhnt und betrachteten die Einfuhrung einer höheren Verwaltungsebene oft als Eingriff in ihr Orts- und Kreis-Furstendasein. Diesem Dilemma versuchte man dadurch zu begegnen, daB bei lokalen Ernennungen und anderen wichtigen personellen Veränderungen das Einverständnis des Senior Military Government Officers vorgeschrieben wurde. Der Militärregierungschef konsultierte dazu seinerseits den Regierungspräsidenten. ${ }^{18}$ Die Entscheidungsgewalt lag jedoch beim Senior Military Government Officer und der lokalen Militärregierung,

14 OMGHE: Historical Report July 1945; Organizational Direction Nr. 2, OMGHE, 24.7.1945; Nr. 3, 26.7.1945; Nr. 5, 31. 7.1945 (8/189-1/5).

15 Chronology, S.3.

16 8/216-3/5.

${ }^{17}$ Die Beziehungen zwischen Regierungsbezirk und Kreis-Militärregierungen regelte Operational Memorandum Nr.1, Det. E1A2, 8.8.1945 (8/216-3/5).

18 Operational Memorandum Nr.3, Det. E1A2, 15.8 .1945 (8/216-3/5). 
wobei der Regierungspräsident ermächtigt war, über Fälle unzureichender Kooperation und mangelnder Leistungsfähigkeit Bericht zu erstatten.

Der Monatsbericht für August 1945 läßt darauf schließen, daß E-5 zu diesem Zeitpunkt über spezialisierte Offiziere für alle wesentlichen Aufgabenbereiche verfügte. Nach und nach folgte die Einteilung der Militärregierung in Divisions. Was die praktische Durchführung von MaBnahmen in den zentralen Problembereichen wie POL (Petrol, Oil, Lubricants) oder Transport betraf, vertraute E-5 in der Anfangsphase zumeist eher auf die Unterstützung der 7 . Armee als auf die eigene Leistungsfähigkeit.

Die Militärregierung verbuchte positiv, daß die politisch „indifferente Haltung“ der Deutschen „aufzutauen begann“. Ihr Bericht vom August informiert sachlich und ohne eigene Meinungsäußerung über die Bestrebungen, drei Parteien im Regierungsbezirk wieder aufzubauen (SPD, KPD und Liberale). Mit der Ablösung des Regierungspräsidenten Hans C. A. F. Bredow und der Ernennung seines Nachfolgers Martin Nischalke am 4. August 1945 setzte die Militärregierung nach eigener Einschätzung dem Einfluß „einiger Industrieller und Kirchenleute“ ein Ende, die zudem gemeint hätten, .,den Amerikanern den rechten Weg und die richtigen Leute zeigen zu müssen" ${ }^{19}$

Am 30. August 1945 versammelte der Senior Military Government Officer, Col. Newman, die Detachment Commanders im Wiesbadener Landeshaus. Er referierte über die Verantwortlichkeit der Militärregierungs-Offiziere gegenüber dem Senior Military Government Officer und informierte über die wichtigsten Ergebnisse eines gerade zu Ende gegangenen Treffens von Militärregierungs-Offizieren in Frankfurt. Wie auch Newmans Vortragsthema zeigte, mußten sich die regionalen Militärregierungen offensichtlich in den ersten Wochen ihrer Tätigkeit erheblich darum bemühen, ihre Macht gegenüber den örtlichen Detachments durchzusetzen, die gemeinsam mit den neuernannten deutschen Amtsinhabern ein Gegengewicht zu den amerikanischen und deutschen Zentralstellen in Wiesbaden bildeten. ${ }^{20}$

Am 8. Oktober 1945 - so vermerkt der Monatsbericht der Militärregierung in militärischer Kürze - wurde aus den Regierungsbezirken Wiesbaden, Hessen (Darmstadt) und Kassel das Land „Groß-Hessen“ gebildet. Demgegenüber notierten dann die Historiographen der Militärregierung nicht ohne Pathos:

„Diese Aufgabe, an der sich schon früher deutsche Regierungen ohne Erfolg versucht hatten, wurde nun von einer siegreichen Macht vollbracht, die jenseits des Atlantiks lag und zu deren Gestade jenes Land einst seine hessischen Soldaten geschickt hatte, um im Unabhängigkeitskrieg zu kämpfen." ${ }^{21}$ E-5 unter Col. James R. Newman wurde nun zum landesweit verantwortlichen Detachment, zum „Office of Military Government for Greater Hesse “ (OMGGH). ${ }^{22}$ Damit war faktisch das für den Regierungsbezirk Wiesbaden zuständige Detachment in OMGGH aufgegangen; formal blieb es aber neben den beiden anderen Regierungsbezirks-Detachments in Kassel und Darmstadt weiter bestehen. Gleichzeitig wurde Wiesbaden zur hessischen Landeshauptstadt bestimmt. ${ }^{23}$

Das im Juli 1945 in Marburg eingesetzte Detachment E-2, das als regionale Militärregierung für das Land Hessen-Nassau hatte arbeiten sollen, wurde wegen seiner

${ }^{19}$ Personnel Directions (ebenda).

20 OMGHE: Historical Report August 1945.

21 OMGHE: Historical Report October 1945.

22 Anweisung des Headquarters Seventh Army, Western Military District: „Organization of Western Military District for Military Government Purposes“ vom 26.9.1945 (5/41-1/20).

${ }^{23}$ Ebenda. 
wenig erfolgversprechenden Entwicklung unter seinem Kommandierenden Offizier Col. Charles T. Johnson zum gleichen Zeitpunkt offiziell dem Kommando von E-5 unter Col. Newman unterstellt. ${ }^{24}$ In der Praxis waren bereits im September 1945 die Zuständigkeiten von E-2 an E-5 übergegangen. Im Laufe des Monats war auch schon die Ausrüstung von E-2 nach Wiesbaden gebracht worden.

\section{Der Aufbau von OMGHE und deutscher Landesregierung}

Im Laufe des Monats Oktober kam es zu verschiedenen organisatorischen Veränderungen innerhalb der Militärregierung. So wurde Detachment E-6 (Frankfurt) zu einem Stadtkreis-Detachment im Regierungsbezirk Wiesbaden. ${ }^{25}$ Folgende Detachments wurden aufgelöst und mit anderen Detachments zusammengelegt:

- im Regierungsbezirk Wiesbaden: H-82 (Weilburg), H-84 (Schlüchtern), H-85 (Idstein/Bad Schwalbach), I-145 (Bad Orb), I-146 (Hadamar), I-147 (Flörsheim) und I-149 (Usingen);

- im Regierungsbezirk Darmstadt: G-37 (Sprendlingen), H-64 (Ober-Ramstadt), I-140 (Beerfelden) und I-141 (?)

- im Regierungsbezirk Kassel: H-66 (Frankenberg), H-74 (Ziegenhain), H-76 (Biedenkopf) und I-144 (Wolfhagen).

Schon im September war ebenfalls die Zusammenlegung einiger Kreisdetachments für November und Dezember 1945 festgelegt worden:

- H-81 Hofheim (Kreis Maintaunus) und H-78 Bad Homburg (Kreis Obertaunus und Usingen);

- H-79 Hanau (Kreis Hanau) und H-78 Gelnhausen (Kreis Gelnhausen und Schlüchtern);

- H-80 Weilburg (Kreis Limburg und Oberlahn) und G-41 Wetzlar (Kreis Wetzlar).

E-5 sollte zum Januar 1946 die Verantwortung für die Kreise Rudesheim, Untertaunus, Maintaunus, Obertaunus, Usingen, Limburg, Oberlahn, Wetzlar und zum Februar für die Kreise Wiesbaden, Hanau, Gelnhausen, Schlüchtern, Dillkreis und Biedenkopf ubernehmen. ${ }^{27}$

Es erwies sich in zahlreichen Fällen als notwendig, trotz Auflösungen und Zusammenlegungen kleinere Büros (Sub-Offices) an den betreffenden Orten aufrecht zu erhalten. Je zwei oder drei Offiziere und einige Soldaten nahmen von Ort zu Ort unterschiedliche Aufgaben wahr, die nicht auf Kreisebene zentralisiert werden konnten. Parallel zur Verkleinerung der Kreis- und Regierungsbezirks-Detachments stieg die Personalstärke von E-5 von 61 Offizieren am 1. Oktober 1945 auf 99 am Ende des Monats.

Vor fast unlösbare Probleme wurde die Militärregierung durch das Military Government Law Nr. 8 vom 26. September 1945 gestellt. Mit diesem juristisch dürftig formulierten, für die Entnazifizierung aber folgenreichen Gesetz war eine drastische Erweiterung des von Entnazifizierungsmaßnahmen betroffenen Personenkreises vorgenommen worden. Auf allen Ebenen sowohl der Militärregierung als auch der

24 Chronology, S.5.

25 Organizational Direction Nr. 11, Det. E-5, 8.10.1945 (8/81-3/21).

26 Kein Nachweis.

27 OMGHE: Historical Report October 1945 (8/216-3/5, 8/216-4/10). 
deutschen Verwaltungsstellen herrschte Unklarheit und Verwirrung. Eines jedoch war sicher: Entnazifizierung war zum „big business“, zur „top-to-bottom affair“ geworden, wie ein zeitgenössischer Beobachter vermerkte. ${ }^{28}$ Die Offiziere waren - selbst überfordert - kaum mehr in der Lage, die von der Bevölkerung gestellten Fragen nach der korrekten Anwendung des Gesetzes zu beantworten.

Andere wichtige Aufgaben konnten dabei ebenfalls nicht aufgeschoben werden: Geeignete Mitarbeiter für die neugeschaffene hessische Regierung muBten gefunden werden. Daneben setzten die Offiziere der Militärregierung ihre bisherigen Tătigkeiten fort. Property Control meldete ebenfalls Probleme: Die Feststellung nationalsozialistischen Eigentums sei noch nicht sehr weit fortgeschritten, da es in vielen Detachments an Engagement in diesem Bereich mangele. Weiter seien die Property Control Officers zu sehr mit anderen Aufgaben beschäftigt und mußten sich überdies beim Aufspüren von Vermögen, das der Kontrolle der Militärregierung zu unterwerfen sei, zu sehr auf Informationen deutscher Zivilangestellter stutzen.

Die MFAA (Museum, Fine Arts and Archives) Section, Economics Division, begann indessen mit dem Aufbau einer Landesorganisation für ihren Bereich. Auch einige andere Abteilungen (DPs, Public Safety u.a.) bezogen bereits statistisches Material aus ganz Hessen in ihren Bericht ein, allerdings ohne diese Kompetenzerweiterung zu thematisieren. Im Informationsbereich spielte DISCC (District Information Services Control Command) als ausfuhrendes Organ der Information Control Division (ICD), USFET, eine wichtige Rolle. ${ }^{29}$

Im Laufe des November 1945 trafen in Wiesbaden immer mehr Militărregierungsoffiziere und deutsche Beamte ein. Das markierte die gestiegene Bedeutung sowohl der amerikanischen als auch der deutschen Zentralregierung. Bereits im Oktober waren die Spitzenpositionen in der deutschen Regierung besetzt worden. Mit den Organizational Directions Nr. 1 vom 12. Oktober 1945 bis Nr. 8 vom 26. Oktober 1945 waren die Hessische Landesregierung eingesetzt sowie die Erlaubnis zur Errichtung der wichtigsten Ministerien erteilt worden. Durch die parallel dazu erlassenen Personnel Directions wurde die personelle Besetzung der Ministerien vollzogen. ${ }^{30}$

Der Monatsbericht vom November zog eine Bilanz des bisher Erreichten. Dabei wurden „Fortschritte“ festgestellt:

„Das beinhaltet den erfolgreichen Aufbau der Landesregierung, den praktischen Abschluß der Entnazifizierung in den Regierungsbehörden, keinen aktiven Widerstand gegen die Besetzung, die Beachtung von Recht und Gesetz durch die Deutschen, das Eintreffen einer bis jetzt nur kleinen Zahl von Flüchtlingen, die zügige Wiederingangsetzung des Kommunikationswesens, die Wiedereinsetzung fast aller Gerichte, die Zulassung von Parteiaktivitäten auf Landesebene mit drei bereits sehr starken und aktiven Parteien, den Abschluß des Wiederaufbaus einer effektiven Landwirtschafts- und Ernährungsbehörde, das Ausbleiben von Epidemien und die Abwendung eines Zusammenbruchs im Gesundheitswesen, die Wiedereröffnung fast aller Grundschulen sowie Fortschritte bei der Wiedereröffnung weiterführender Schulen." 31

Schlechter sah die Bilanz auf dem Gebiet der Wirtschaft aus: Geringe industrielle Aktivität, mangelhafte Versorgung, fehlende Ausrüstung, Konsumgüter- und Baumate-

28 Freeman: Hesse.

29 OMGHE: Historical Report October 1945.

30 OMGHE: Historical Report October 1945 - June 1946, Appendix; Organizational Directions and Personnel Directions auch in 8/216-3/4.

${ }^{31}$ OMGHE: Historical Report November 1945. 
rialknappheit bestimmten das Dasein der deutschen Bevölkerung. Dazu versprach der Bericht, daß man sich von seiten der Militärregierung - so gut es die bestehenden Verhältnisse zuließen - mit den Schlüsseln zur Lösung dieser Probleme beschäftigte, nämlich mit der Kohleversorgung und dem Transportwesen.

Schlaglichtartig seien im folgenden weitere Entwicklungen skizziert. Mit der Information Control Instruction Nr. 2 wurde den Parteien im November erlaubt, publizistisch tätig zu werden. Insgesamt bestanden im November bereits 133 Kreis-Parteiorganisationen (in 39 von 41 Kreisen); SPD und KPD waren in je 39 Kreisen organisiert.

Die Gerichte der Militärregierung gingen hart gegen Waffenbesitz, Manipulationen an Fragebogen sowie widerrechtlichen Besitz amerikanischer Güter vor (z. B. in Frankfurt: 6 Jahre Haft wegen unberechtigten Besitzes eines Gewehres). Im Sicherheitsbereich ergaben sich zunehmend Probleme durch bewaffnete Banden von DPs. Die Verstärkung der deutschen Polizeikräfte sollte hier Abhilfe schaffen. Der interzonale Postverkehr, im Oktober in die Wege geleitet, stieg sprunghaft an. ${ }^{32}$

Am 15. November 1945 wurde das 2nd Military Government Battalion (Separate), 2nd MGBat(Sep), aufgestellt. Kommandeur dieser Einheit war in Personalunion der Direktor von OMGHE, der gleichzeitig auch noch als Direktor der Militärregierung im Regierungsbezirk Wiesbaden fungierte. ${ }^{33}$ Das 2nd MGBat(Sep) hatte für die MG-Detachments Verwaltungs- und Versorgungsaufgaben wahrzunehmen. Zum 30. November gehörten ihm und seinen untergeordneten Einheiten 622 Offiziere und 920 Soldaten an. Seine Büros hatte das 2 nd MGBat(Sep) im Wiesbadener Landeshaus bezogen. Im November 1945 wurden die Detachments G-36 (GroB-Gerau), H-59 (Alsfeld), H-60 (Erbach), H-69 (Hünfeld), H-70 (Melsungen), H-71 (Rotenburg) und H-73 (Witzenhausen) aufgelöst. Im Regierungsbezirk Kassel fand eine umfassende Neuordnung des Detachment-Systems statt. Mit Ausnahme von G-39 (Marburg) kontrollierte dort jetzt jedes Detachment je zwei Kreise.

Der letzte Monat des Jahres 1945 brachte „grundlegende Veranderungen in der Organisation der Militärregierung in Groß-Hessen mit sich. Fast alle Aufgaben, für die zuvor die Kreis- und Regierungsbezirks-Detachments zuständig gewesen waren, wurden auf die deutsche Zivilregierung ubertragen. ${ }^{344}$ Mit der Organizational Direction Nr. 10 vom 15. Dezember 1945 wurden den Regierungsbezirks-Detachments auf Grundlage einer Anweisung von USFET ${ }^{35}$ wesentliche Befugnisse im Finanz-, Justiz- und Sozialbereich entzogen. Parallel dazu ermächtigte die Organizational Direction Nr.11 vom 24. Dezember 1945 den Ministerpräsidenten, die entsprechenden Abteilungen der Bezirksregierungen aufzulösen und deren Aufgaben auf Landesbehörden zu übertragen. Weitere Directions $(12,13,14,16)$ entzogen den Kreis- und Regierungsbezirksdetachments neben vielen anderen Aufgaben ihre Ernennungsbefugnisse und übertrugen sie auf den Ministerpräsidenten. Die Organizational Direction Nr. 19 vom 20. Juni 1946 gab diese Rechte an die inzwischen gewählten Orts- und Kreisparlamente weiter. ${ }^{36}$ Vor diesen Zentralisierungs- und Demokratisierungsmaßnahmen hatte sich ,jeder Chef einer örtlichen Dienststelle als omnipotenter Besitzer eines politischen Schrebergartens" betrachtet, wie Samuel $\mathrm{L}$. Wahrhaftig in seinen Erinnerungen an die Besatzungszeit in Hessen festhielt. ${ }^{37}$

\footnotetext{
32 Ebenda; OMGHE: Historical Report December 1945.

33 General Order Nr. 1, Hq 2nd MGBat(Sep), 15.11.1945.

34 OMGHE: Historical Report December 1945.

35 OMGHE: Historical Report October 1945 - June 1946.

36 Ebenda, Appendix; Organizational Directions in 8/216-3/5 und 8/216-3/4.

37 Wahrhaftig: In jenen Tagen, S. 787.
} 
Mit General Order Nr.337 vom 14. Dezember 1945 des Headquarters US Forces, European Theater (USFET) wurde dem Western Military District und seinen Armeen die Kontrolle über die Landesmilitärregierung entzogen. Die Militärverwaltung behielt die Kriegsgerichtsbarkeit (general court martial jurisdiction) und die Hoheit in einigen militärischen und Sicherheitsfragen. Die Landesmilitärregierung war jetzt USFET gegenüber direkt verantwortlich. Sie konnte jedoch auch Anweisungen vom Office of Military Government for Germany, United States (OMGUS), in Berlin und vom Office of Military Government for Germany, US-Zone (OMGUSZ), in Frankfurt erhalten. ${ }^{38}$ Die dadurch geschaffene unklare Befehlssituation, die Kompetenzstreitigkeiten innerhalb der amerikanischen Regierung zum Ausdruck brachte, ${ }^{39}$ beendete USFET mit General Order Nr.61 vom 7. Mărz 1946. Diese Anordnung löste OMGUSZ auf und übertrug seine Aufgaben an OMGUS. Die Ländermilitärregierungen wurden unter Aufsicht des für OMGUS verantwortlichen stellvertretenden Militärgouverneurs der Vereinigten Staaten in Deutschland gestellt. Die von General Order Nr.337 festgelegte Hoheit der Armee in juristischen und militärischen Fragen blieb erhalten. Armee und Militärregierung wurden darin weiter zu einer engen Zusammenarbeit in der Nachrichtenbeschaffung, technischen Hilfe $u$. a. verpflichtet. ${ }^{40}$

Ubersicht 2: Entwicklung der Befehlskette zwischen der amerikanischen Militärregierung in Hessen und ihren vorgesetzten Dienststellen, Oktober 1945 bis April 194641

\section{CHAIN OF COMMAND}

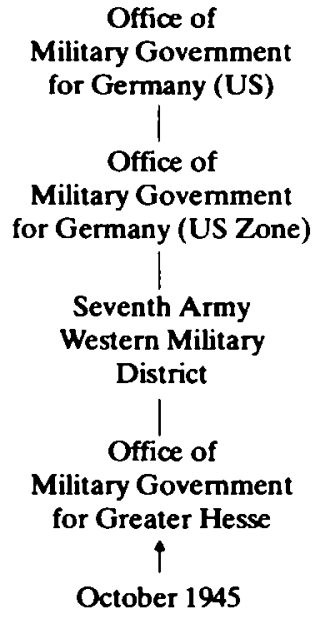

Office of

Military Govemment

for Germany (US)

Office of

Military Govemment

for Germany (US Zone)

Seventh Army

Western Military

District

Office of

Military Govemment

for Greater Hesse

October 1945

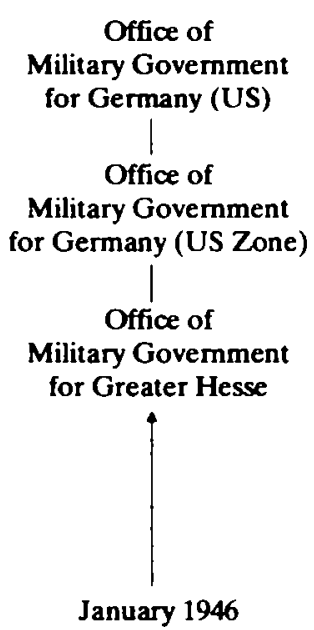

Office of

Military Govemment

for Germany (US)

Office of

Military Government

for Greater Hesse

1

April 1946

38 General Order Nr. 337, USFET, 14.12.1945 wurde am 19.2.1946 durch General Order Nr. 43, USFET, ergänzt. Beide Orders in: 12/226-1/1-11 (26 of 54); vgl. auch 8/179-1/8. Der mit General Order Nr. 188, USFET, 12.8.1945 eingerichtete Western Military District wurde mit General Order Nr. 1, USFET, 2.1.1946, wieder aufgelöst. An die Stelle des Hessen umfassenden Districts trat die 7. US-Armee. Diese beendete mit General Order Nr.66, USFET, 13.3.1946, ihre Tätigkeit (vgl. 12/226-1/1-11 (26 of 54)).

$39 \mathrm{Vgl}$. Ziemke: Oocupation of Germany, vor allem S. $401 \mathrm{ff}$.

$4012226-1 / 1-11$ (26 of 54).

41 OMGHE: Historical Report October 1945 - June 1946, Appendix. 
Eine entscheidende Veränderung in Struktur und Organisation der Militärregierung markierte die Auflösung aller Detachments durch General Order Nr. 14 vom 28. Mai 1946. An die Stelle der Detachments traten Liaison \& Security Offices (LSOs, ungefähr: Verbindungs- und Sicherheitsbüros) in allen Land- und Stadtkreisen, die bereits mit General Order Nr. 13 vom 21. Mai 1946 eingerichtet worden waren. Neben ihren „Verbindungs“-Aufgaben hatten sich die LSOs um die öffentliche Sicherheit, die Entnazifizierung und die Militärgerichtsbarkeit zu kümmern.

Auf Landesebene trat mit General Order Nr. 15 vom 29. Mai 1946 das Office of Military Government for Greater Hesse (OMGGH), das zum 3. Juni 1946 innerhalb des 2 nd MGBat(Sep) gebildet wurde, offiziell an die Stelle des Detachments E-5. ${ }^{42}$ Am 8. April wurde die Bezeichnung dann in „Office of Military Government for Hesse“ (OMGHE) umgeändert.

Diese organisatorischen Veränderungen, die Col. Newman bereits am 11. Februar 1946 in einer Denkschrift an den Stellvertretenden Militärgouverneur, Lucius D. Clay, in Grundzilgen formuliert hatte, brachten den Funktionswandel der Militärregierung zum Ausdruck: An Stelle direkter Eingriffe und eigener Mitwirkung wurden die deutschen Exekutivorgane nun beaufsichtigt und kontrolliert. Diese deutschen Verwaltungseinrichtungen konnten seit der Durchführung von Wahlen (Gemeindewahlen am 20/27. Januar 1946, Kreistagswahlen am 28. April 1946, Wahlen in den Stadtkreisen am 26. Mai 1946) auch demokratische Legitimation beanspruchen.

Auf Landesebene erfolgten die Wahlen zur Verfassungberatenden Landesversammlung am 30. Juni 1946. In der Versammlung verfügten die SPD uber 42, die CDU über 35, die KPD uber sieben und die LDP uber sechs Abgeordnete. Die Landtagswahl vom 1. Dezember 1946, bei der gleichzeitig die Landesverfassung zur Abstimmung gestellt wurde, brachte der SPD 38, der CDU 28, der LDP 14 und der KPD zehn Sitze.

An die Stelle der von dem parteilosen Karl Geiler gefuhrten Landesregierung trat am 3. Januar 1947 eine Koalitionsregierung aus SPD und CDU unter dem Ministerpräsidenten Christian Stock (SPD). Obwohl die Militärregierung Präferenzen für den von ihr ausgewählten und eingesetzten Geiler hatte, muBte sie den Personalvorschlägen der durch demokratische Wahlen legitimierten Parteien zustimmen, wollte sie nicht ihren eigenen Grundsätzen untreu werden. ${ }^{43}$

Der VerfassungskompromiB von CDU und SPD fand nach einigen Veränderungen die Zustimmung von OMGHE und OMGUS. Auf Verlangen von OMGUS muBte der Artikel41 einer gesonderten Volksabstimmung unterworfen werden. Dieser Artikel sah die Sofortsozialisierung von Bergbau-, Energieversorgungs-, eisen- und stahlerzeugenden Unternehmen sowie von bestimmten Verkehrsbetrieben vor. Im Volksentscheid vom 1. Dezember 1946, gleichzeitig mit der Landtagswahl und der Abstimmung uber die Annahme der Verfassung durchgefürt, wurde der Artikel mit 72 Prozent der Stimmen angenommen. Die Landesregierung setzte daraufhin die Besitzer der somit „sozialisierten" Betriebe als Treuhänder ein. Die praktische Umsetzung der von SPD und Teilen der CDU entwickelten Sozialisierungsmodelle scheiterte schlieBlich an Unstimmigkeiten innerhalb der SPD/CDU-Koalition, am hinhaltenden Widerstand der Militärregierung und an juristischen Schritten, die die oppositionelle LDP/FDP einleitete. ${ }^{44}$ Zur Entspannung des Verhältnisses zwischen Militärregierung und deutscher Bevölkerung trug die Aufhebung des Ausgehverbots im April 1946 bei. $^{45}$

\footnotetext{
42 Ebenda; auch 5/41-1/20.

43 Wahrhaftig: In jenen Tagen, S. 104.

${ }^{44}$ Kropat: Hessen in der Stunde Null, S. 121, $260 \mathrm{ff}$.

$458 / 53-1 / 26$.
} 
Mit USFET Circular Nr. 71 vom 25. Mai 1946 wurden Resource Boards eingerichtet und deren Aufgaben definiert. Angesichts der enormen Versorgungsprobleme auf allen Ebenen sollten die Boards als Koordinationsgremien zwischen Armee und Militärregierung für beide Seiten zufriedenstellende Lösungen finden. In Hessen wurde 1947 ein Land Resource Board ins Leben gerufen, der von sechs Local Resource Boards unterstützt wurde. Die Boards waren bei den Military Posts (amerikanische Militärstandorte) angesiedelt und bestanden aus je einem Vertreter der Militärregierung und der Armee. ${ }^{46}$

\section{Organisation und Entwicklung der amerikanischen Militärregierung in Hessen}

Für personelle Kontinuität in der hessischen Militärregierung sorgte James $\mathbf{R}$. Newman, ein „früherer West Pointer“ und Kenner der Army, der der hessischen Militärregierung von Beginn an bis zur Auflösung als Direktor vorstand.

In Command \& Executive (Kommando und Exekutive: Stabsabteilung) wurde der Direktor von Anfang an von einem Deputy Director unterstützt, der als Deputy Director for Operations bis Juni 1946 für die Kontrolle der LSOs zuständig war. Nach der Eingliederung der früheren SHAEF Enklave Frankfurt im September 1945 war der im November dieses Jahres eingesetzte zweite Deputy Director for Frankfurt zuständig. Diese Stelle entfiel jedoch am 13. Oktober 1947, als der Stelleninhaber (Robert K. Phelps) zum Bipartite Control Office versetzt wurde. ${ }^{47}$

Eine wichtige Rolle im Verwaltungsgang spielten der Executive Officer und der Assistant Executive Officer. Beide waren im internen Befehlsstrang zeichnungsberechtigt (wie auch der Deputy Director). ${ }^{48}$ Zunächst hatten diese beiden Offiziere an der Spitze der Befehlspyramide im 2 nd MGBat(Sep) gestanden, wurden aber mit dem Aufbau von OMGHE auch den Divisions übergeordnet. Nach der Umstrukturierung in der ersten Jahreshälfte 1947 erhielt das Battalion im Juli einen Director of Administrative Services, was auch die geringer werdende Bedeutung militärischer Elemente in der Militärregierung andeutet. Für Personalangelegenheiten hatte bis März 1946 (Einrichtung einer eigenen Personnel Division) der Adjutant im 2 ndMGBat(Sep) die Hauptzuständigkeit. Er verfügte über ein eigenes Büro, das bis zu zehn Mitarbeiter umfaßte.

Am Tag der Aufstellung (15.September 1944) gehörten dem späteren Regierungsdetachment E-5 114 Offiziere und 36 Mann an. ${ }^{49}$ Ein Jahr später, am 15. November 1946, waren es bereits 114 Offiziere und 52 Mannschaften..$^{50}$ Im Juni 1946 betrug die Zahl des US-Personals (Offiziere, Soldaten und Zivilisten) in der Militärregierung für Hessen 618. ${ }^{51}$ Diese Zahl sank kontinuierlich, während sich die Zahl der deutschen Mitarbeiter der Militärregierung zunächst deutlich erhöhte. So arbeiteten im August 1946609 Amerikaner und 2196 Deutsche in OMGGH. ${ }^{52}$ Bis zum Dezember war der

46 OMGHE: Historical Report April - July 1947, Appendix.

47 OMGHE: Historical Report October - December 1947. Dort wird das „Office“ noch als "Council" bezeichnet.

48 8/193-2/4.

$498 / 187-1 / 2$.

50 OMGHE: Historical Report November 1945.

51 8/179-3/8.

$528 / 179-3 / 6$. 
Personalstand der Deutschen auf 2997 angewachsen, während die Zahl der Amerikaner nur noch 545 betrug. ${ }^{53}$

Diese Verschiebungen sind Ausdruck des „ever current personnel problem“, von dem im zweiten großen Historical Report die Rede ist. ${ }^{54}$ Das Personalproblem zeigte sich einerseits in der starken Fluktuation und andererseits im ständigen Mangel an qualifiziertem Personal. Die übliche „Überseedienstzeit“ für US-Soldaten dauerte 30 Monate. Nur wenige Mitarbeiter der Militärregierung verlängerten ihre Dienstzeit auf 36 Monate. Diesen Schwierigkeiten versuchte OMGUS mit einem Civilianization Program zu begegnen: Durch AnschluBarbeitsverträge auf Angestelltenbasis sollten qualifizierte Mitarbeiter zu einem längeren Verbleiben in Deutschland gebracht werden. 55

Im Juni 1947 startete OMGHE ein Ausbildungsprogramm, das amerikanische Universitätsabsolventen auf eine Tătigkeit in der Militärregierung vorbereiten sollte. ${ }^{56}$ Trotz dieser Maßnahmen blieb der Personalmangel bestehen; oft konnten nicht einmal alle zur Verfügung stehenden Stellen besetzt werden. In einzelnen Făllen versuchte die Militärregierung, durch Beantragung der Hohergruppierung von Stellen in besonders wichtigen Bereichen (z.B. Press Branch) besser qualifiziertes Personal zu bekommen. ${ }^{57}$ RegelmăBig gab es Unstimmigkeiten zwischen OMGUS und OMGHE, wenn die Personalzuweisung durch die vorgesetzte Dienststelle nicht den Wüschen von OMGHE entsprach..$^{58}$ Diese Probleme fuhrten dazu, daB in den OMGUS-Akten widersprichliche Angaben uber Personalstärke und -ausstattung keine Seltenheit sind. Weitere Ursachen für die Personalprobleme sind in der starken internen Fluktuation in OMGHE und auch in Unklarheiten im Verhaltnis zwischen OMGHE und dem 2 nd MGBat(Sep) zu suchen. ${ }^{59}$ So geben die folgenden Balkendiagramme zwar durchaus richtige Tendenzen der Personalentwicklung wieder, stimmen aber nicht genau mit den an anderen Stellen genannten Zahlen uberein.

53 8/179-3/4.

${ }^{54}$ OMGHE: Historical Report July - December 1946.

55 Ebenda.

56 OMGHE: Historical Report April - July 1947.

57 8/179-3/4.

58 So z. B. Legal Division, ebenda.

59 OMGHE: Historical Report October 1945 - June 1946. 


\section{Ubersicht 3: Personalentwicklung von OMGHE, November 1945 - Juni $1946^{60}$}

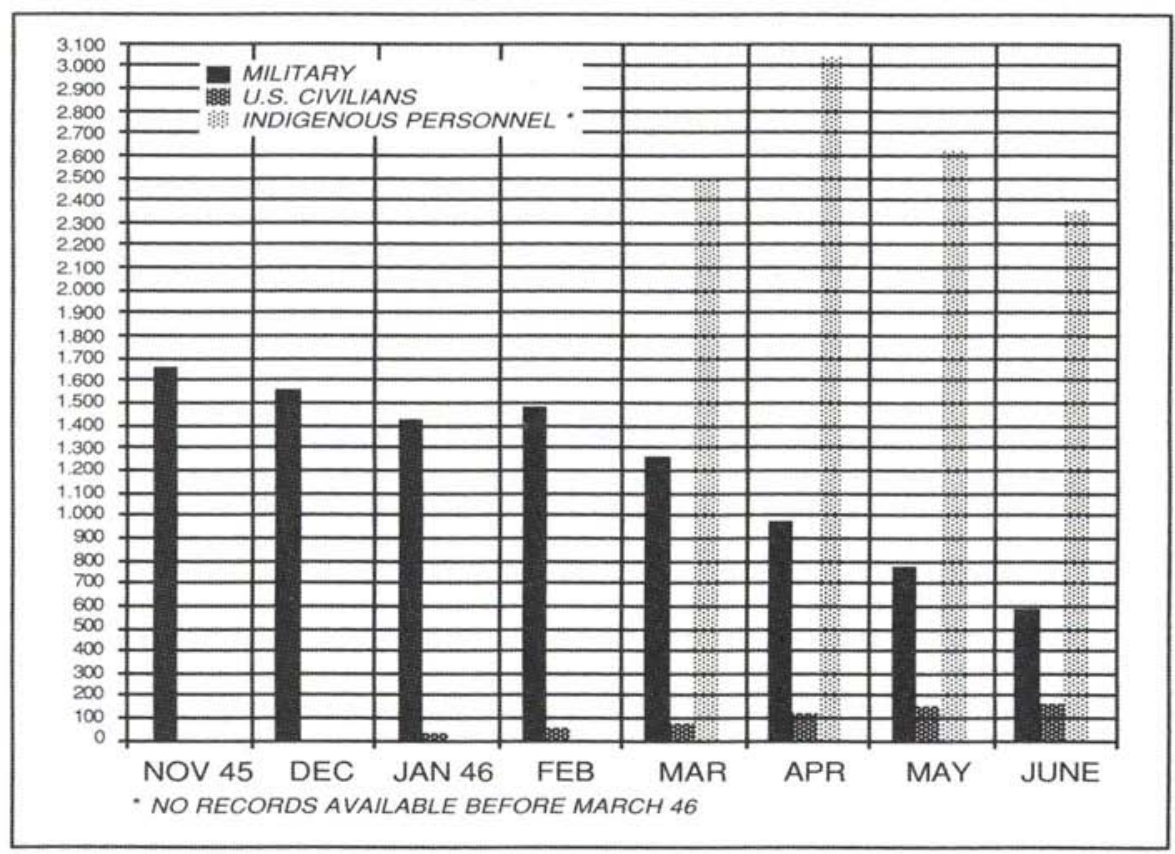

Ubersicht 4: Personalentwicklung von OMGHE, Juli 1946 - Juni $1947^{61}$

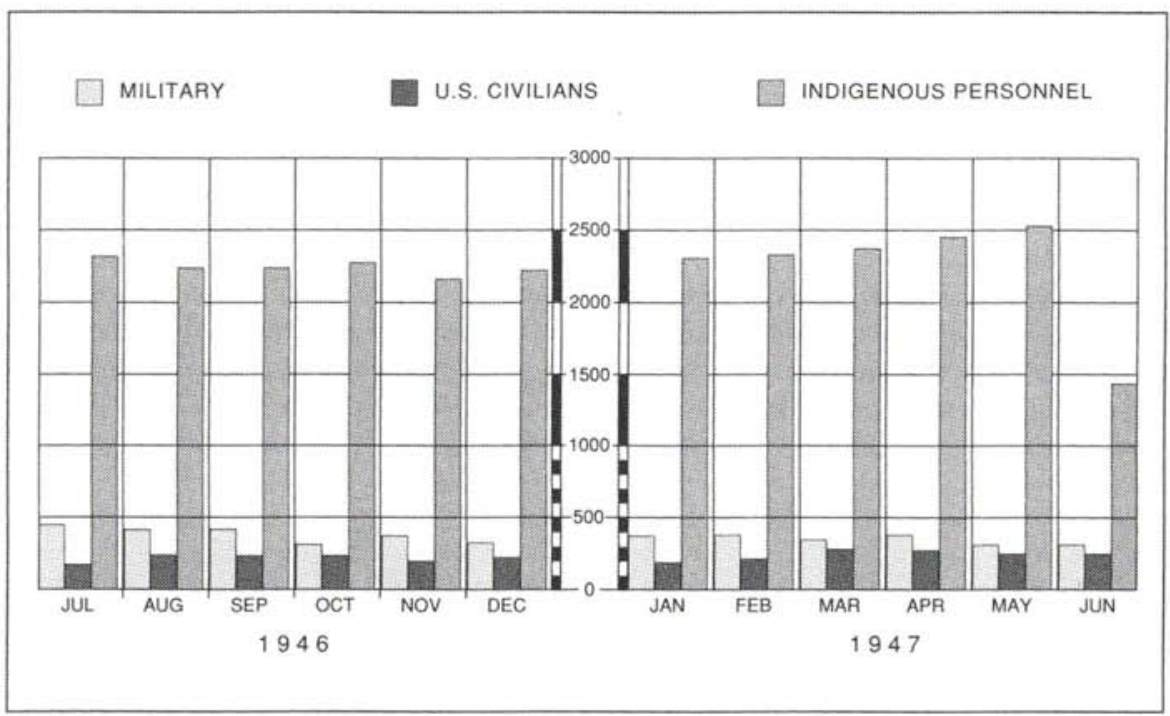

60 S/41-1/19.

61 OMGHE: Historical Report April - July 1947, Annex L. 
Eine Verringerung der Personalstärke um 42\% trat im zweiten Vierteljahr 1947 ein. als die Versorgungsaufgaben (housekeeping activities) von OMGHE an die Military Posts, d.h. die amerikanischen Militärstandorte, übertragen wurden. Das amerikanische Personal verringerte sich von 530 am 30. April 1947 auf 443 am 30. Juni 1947. Noch einschneidender war die Veränderung bei den deutschen Stellen. Ihre Zahl sank von 2583 am 30. April 1947 auf 1360 am 30. Juni 1947. Fast alle bisher bei OMGHE Beschäftigten wurden jedoch von den Military Posts übernommen. ${ }^{62}$ Im Zuge der fortschreitenden Abgabe von Kompetenzen an deutsche Stellen verringerte sich das Personal der Militärregierung kontinuierlich, so zum Beispiel durch den Rückzug der Militärregierung aus der Entnazifizierung im Sommer 1948. Schon am 22. Januar 1948 hatte OMGUS angekündigt, das US-Personal der Militärregierung in Deutschland um 4000 Stellen zu kürzen. ${ }^{63}$

Für Hessen ergaben sich folgende Zahlen (Personalzuweisungen durch OMGUS): ${ }^{64}$

Ubersicht 5: Personalzuweisungen an OMGHE durch OMGUS, Januar 1948 - September 1949

US-Personal Deutsches Personal

$\begin{array}{rrr}\text { 22. } 1.1948 & 392 & \\ \text { 5. } 3.1948 & 234 & \\ \text { 14. } 5.1948 & 231 & 872 \\ \text { 5. } 6.1948 & 232 & 801 \\ \text { 23. } 8.1948 & 229 & \\ \text { 24. } 9.1948 & 202 & \\ 4.10 .1948 & 210 & 460 \\ 23.11 .1948 & 214 & 460 \\ 15.1 .1949 & 202 & 460 \\ 28.2 .1949 & 183 & 488 \\ \text { 31. } 5.1949 & 175 & 488 \\ \text { 30. } 6.1949 & 168 & 492 \\ 31.7 .1949 & 140 & \\ 31.8 .1949 & 130 & \\ 20.9 .1949 & 129 & \end{array}$

Der Anteil von Militärs am Personal der Militärregierung sank bis zum 31. Juli 1949 auf Null. In den letzten Monaten amtierte eine völlig zivile „Militär“-Regierung.

Bis Juni 1946 war die Organisationsstruktur von OMGHE so ausgebaut, daß sie in ihren Grundzügen bis 1948 bestehen bleiben konnte. Die Übersicht 6 zeigt gleichzeitig die Zuständigkeiten für die deutschen Verwaltungseinheiten, die von den Abteilungen der Militärregierung (Divisions) kontrolliert wurden.

62 OMGHE: Historical Report April - July 1947, S. 3.

63 8/179-3/4.

${ }^{64}$ Bis 23.11.1948: 8/179-3/4, 15.1. und 28.2.1949: 5/42-3/9 (assigned personnel), 31.5. und 30.6.1949: 5/42-3/10 (assigned personnel), 31.7. - 20.9.1949: 5/42-3/11 (assigned personnel). 


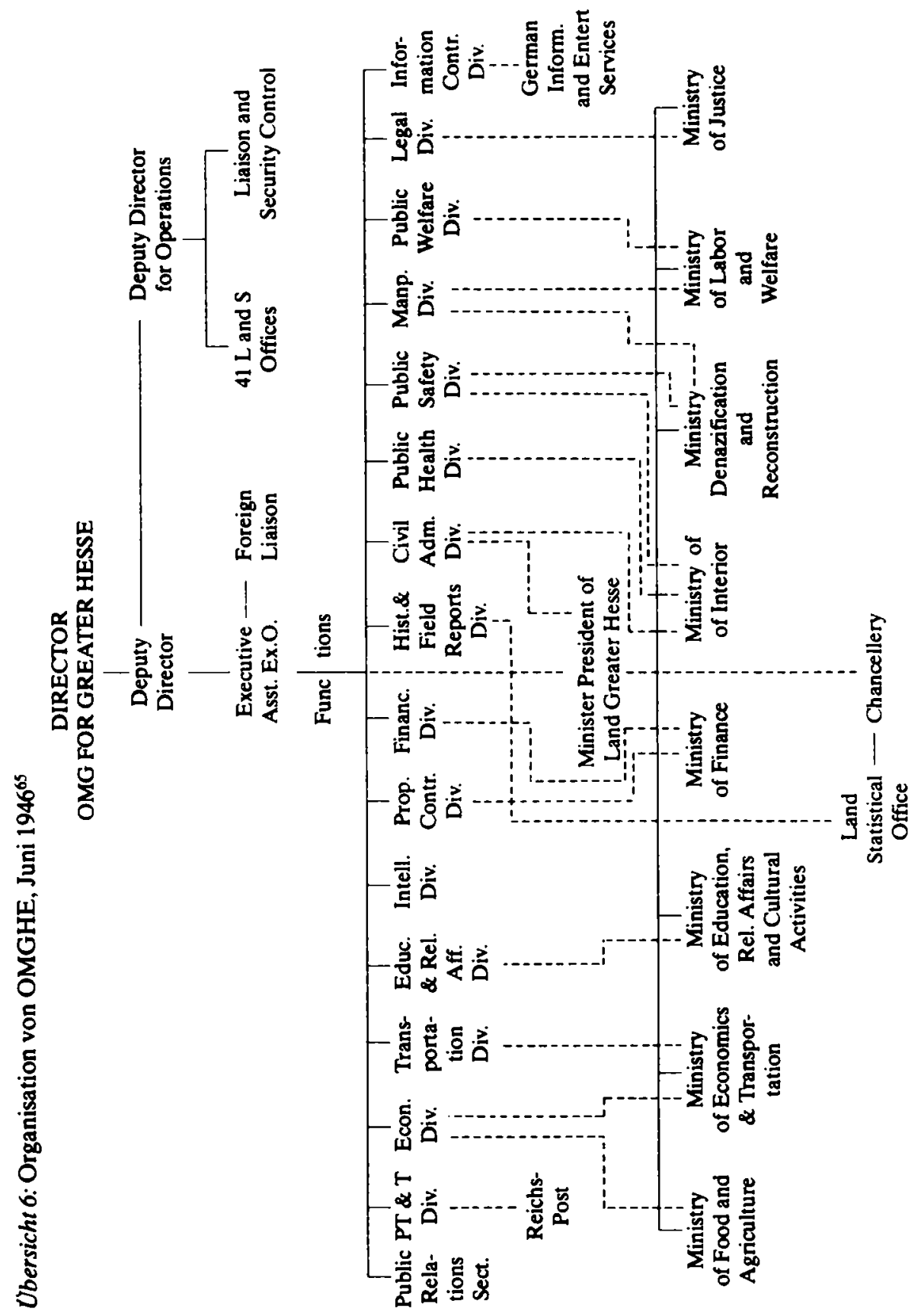

${ }^{65}$ OMGE: Historical Report October 1945 - June 1946. 
Im Juli 1946 wurde eine eigenständige Denazification Division eingerichtet. ${ }^{66}$ Zum 31. Dezember 1946 stellte die Post, Telephone and Telegraph Division ihre Tätigkeit ein. Ihre Aufgaben - sofern sie nicht auf die Deutsche Post übertragen worden waren - übernahm die Communication Branch, OMGUS. ${ }^{67}$

Die folgende Übersicht vom Juni 1947 zeigt die Organisationsstruktur der Militärregierung nach diesen Veränderungen.

Ubersicht 7: Organisation von OMGHE und hessischer Regierung, Juni $1947^{68}$

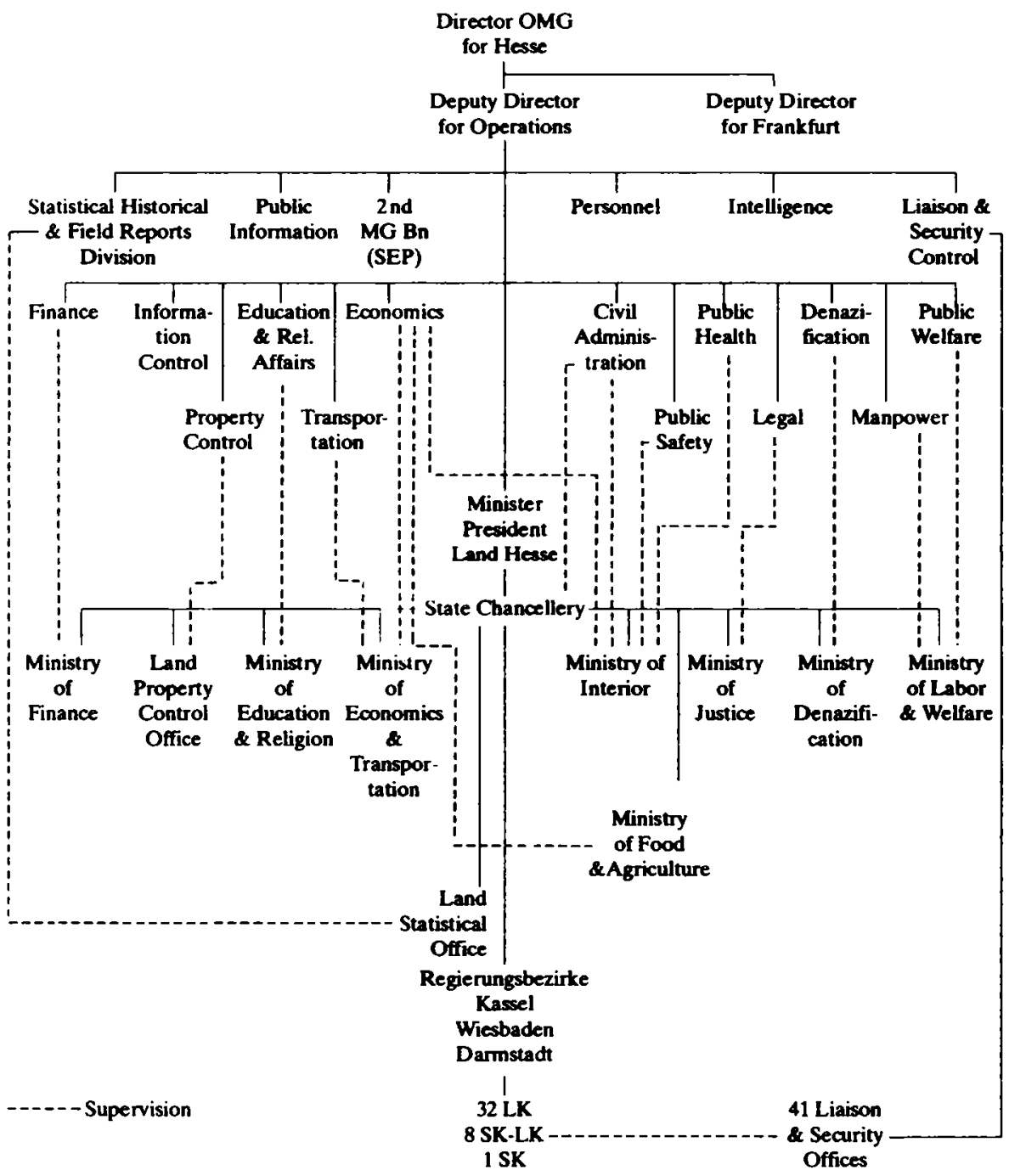

${ }^{66}$ Chronology, S. 23.

67 OMGHE: Historical Report July - December 1946.

68 5/41-27. 
Ende 1947 entfiel die Stelle des Deputy Director for Frankfurt. Für Administrative Services and Foreign Liaison wurden jetzt eigene Abteilungen eingerichtet. Die Administrative Services übernahmen gemeinsam mit der Personnel Division von dem in Auflösung begriffenen 2 nd MGBat(Sep) die Verwaltungsaufgaben. In der inneren Verwaltung von OMGHE stellten sie keine eigenständige Einheit dar. Foreign Liaison faBte die Offiziere, die für den Kontakt mit anderen Staaten und deren DPs zuständig waren, zusammen.69 Dadurch ergab sich zum 1. Januar 1948 folgende Organisationsstruktur.

Ubersicht 8: Organisation von OMGHE, Januar $1948^{70}$

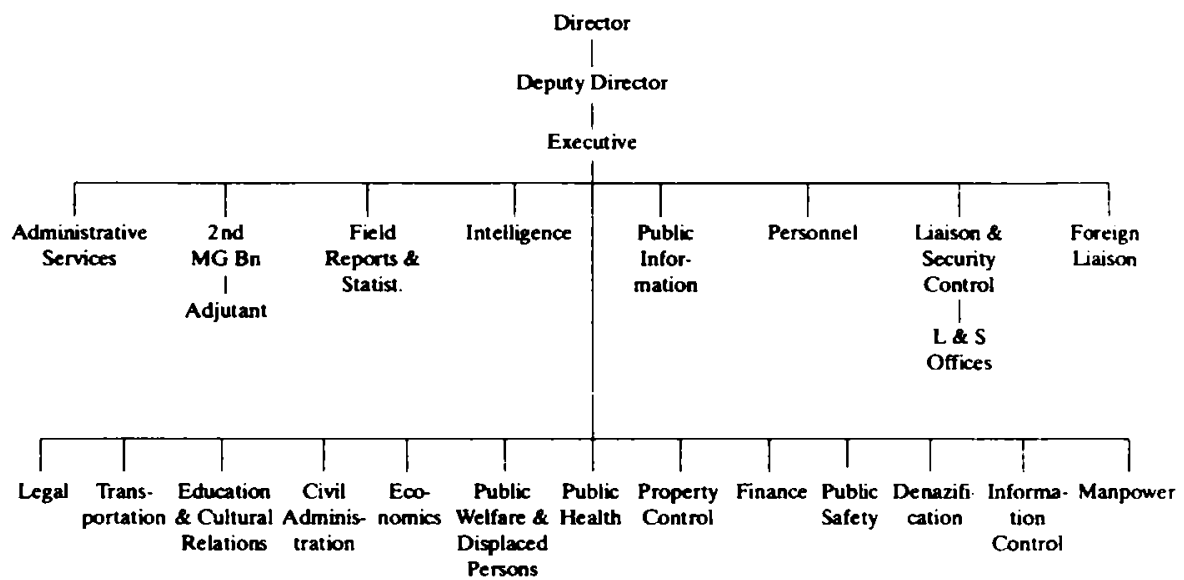

Mit General Order Nr. 4 vom 1. März 1948 strukturierte die Militärregierung die Education and Religious Affairs Division in Education and Cultural Relations Division um. ${ }^{71}$ Im April 1948 kam es zu einer deutlichen Straffung des Organisationsaufbaus. General Order Nr. 5 vom 20. April 1948 ${ }^{72}$ - ein Befehl, der uns bei der Beschreibung der einzelnen Divisions und Branches noch häufig begegnen wird - löste folgende Divisions auf: Civil Administration, Public Health, Public Safety und Public Welfare \& Displaced Persons. Ihre Aufgaben wurden der neu gebildeten Civil Administration Division übertragen, die sich nun aus den Branches Government Affairs, Public Health, Public Safety und Public Welfare \& DPs zusammensetzte.

Weiter löste General Order Nr. 5 auch die Economics, Transportation, Manpower, Finance und Property Control Divisions auf. Die Aufgaben der Transportation, der Manpower und der Finance Division sowie der Food and Agriculture Branch - einer Abteilung der aufgelösten Economics Division - wurden auf die entsprechenden Branches der neu eingerichteten Bizonal Liaison Division übertragen, die bis auf die Bereiche Decartelization, Reparation, Restitutions und Monuments, Fine Arts \& Archives auch alle anderen Aufgaben der Economics Division übernahm. Die noch

${ }^{69}$ OMGHE: Historical Report October - December 1947.

${ }^{70} 5 / 41-3 / 10$.

${ }^{71}$ Ebenda.

$728 / 215-1 / 3$, auch in 5/41-3/10. 
verbliebenen Aufgabenfelder übernahm die neu geschaffene Property Division, die darüber hinaus für Auditing and Accounts und die Aufgaben der Property Control zuständig war. ${ }^{73}$

Diese Straffung war einerseits durch die Übertragung von Aufgaben an deutsche Behörden ermöglicht worden und andererseits Folge der Errichtung des Vereinigten Wirtschaftsgebietes (Bizone), die die USA und Großbritannien am 2. Dezember 1946 beschlossen hatten.

Ubersicht 9: Organisation von OMGHE, 30. April 1948 ${ }^{74}$

\begin{tabular}{|c|c|c|c|c|c|c|}
\hline \multicolumn{7}{|c|}{ Director } \\
\hline $\begin{array}{c}7780 \\
\text { OMGUS Grp. } \\
\text { Hesse Sec. }\end{array}$ & $\begin{array}{c}\text { Intelligence } \\
\text { Divison }\end{array}$ & $\begin{array}{l}\text { Hist. Field } \\
\text { Reports }\end{array}$ & & $\begin{array}{c}\text { Public } \\
\text { Information }\end{array}$ & $\begin{array}{c}\text { Personnel } \\
\text { Division } \\
\text { Administrative } \\
\text { Branch } \\
\text { Accomo- } \\
\text { dations } \\
\text { Branch }\end{array}$ & $\begin{array}{c}\text { L \& S Control } \\
\text { Division } \\
\mid\end{array}$ \\
\hline $\begin{array}{l}\text { Property } \\
\text { Division }\end{array}$ & $\begin{array}{c}\text { Legal } \\
\text { Division } \\
\mid\end{array}$ & $\begin{array}{c}\text { Education } \\
\text { \&Cult. } \\
\text { Relations } \\
\text { Division } \\
\mid\end{array}$ & $\begin{array}{c}\text { Denazifi- } \\
\text { cation } \\
\text { Division }\end{array}$ & $\begin{array}{c}\text { Information } \\
\text { Control } \\
\text { Division } \\
\mid\end{array}$ & $\begin{array}{c}\text { Civil } \\
\text { Adminis- } \\
\text { tration } \\
\text { Division } \\
\text { I }\end{array}$ & $\begin{array}{l}\text { Bipartite } \\
\text { Liaison } \\
\text { Divition } \\
\mid\end{array}$ \\
\hline $\begin{array}{c}\text { Auditing \& } \\
\text { Accounts } \\
\text { Branch }\end{array}$ & $\begin{array}{l}\text { Prisons } \\
\text { Branch }\end{array}$ & $\begin{array}{c}\text { General } \\
\text { Education } \\
\text { Branch }\end{array}$ & & $\begin{array}{c}\text { Radio } \\
\text { Branch } \\
\mid\end{array}$ & $\begin{array}{c}\text { Government } \\
\text { Affairs } \\
\text { Branch } \\
\end{array}$ & $\begin{array}{c}\text { Economics } \\
\text { Branch }\end{array}$ \\
\hline $\begin{array}{c}\text { Property } \\
\text { Branch }\end{array}$ & $\begin{array}{c}\text { Military } \\
\text { Governments } \\
\text { Branch }\end{array}$ & $\begin{array}{l}\text { Higher } \\
\text { Education } \\
\text { Branch }\end{array}$ & & $\begin{array}{c}\text { Press } \\
\text { Branch }\end{array}$ & $\begin{array}{c}\text { Public } \\
\text { Safety } \\
\text { Branch }\end{array}$ & $\begin{array}{c}\text { Transportation } \\
\text { Branch }\end{array}$ \\
\hline $\begin{array}{c}\text { Decarteliza- } \\
\text { tion } \\
\text { Branch } \\
\end{array}$ & $\begin{array}{l}\text { Administration } \\
\text { of German } \\
\text { Justice, } \\
\text { Legislation \& } \\
\text { Legal } \\
\text { Branch }\end{array}$ & $\begin{array}{c}\text { Adult \& } \\
\text { Vocational } \\
\text { Education } \\
\text { Branch } \\
\end{array}$ & & $\begin{array}{c}\text { Publications } \\
\text { Branch }\end{array}$ & $\begin{array}{c}\text { Public Health } \\
\text { Branch }\end{array}$ & $\begin{array}{c}\text { Food \& } \\
\text { Agriculture } \\
\text { Branch }\end{array}$ \\
\hline $\begin{array}{c}\text { Reparations } \\
\text { Branch } \\
\substack{\text { Monuments \& } \\
\text { Fine } \\
\text { Arts Branch }}\end{array}$ & & $\begin{array}{l}\text { Religious } \\
\text { Affairs } \\
\text { Branch } \\
\text { | } \\
\text { Youth } \\
\text { Activities } \\
\text { Branch }\end{array}$ & & $\begin{array}{c}\text { Film, Theater, } \\
\text { Music } \\
\text { Branch } \\
\text { ! } \\
\text { Information } \\
\text { Center } \\
\text { Branch }\end{array}$ & $\begin{array}{c}\text { Public Welfare } \\
\text { Branch }\end{array}$ & $\begin{array}{c}\text { Manpower } \\
\text { Branch }\end{array}$ \\
\hline
\end{tabular}

${ }^{73} \mathrm{Vgl}$. die entsprechenden Kapitel OMGUS-Hb, Teil Hessen B.

74 5/41-3/10. 
Mit General Order Nr.9, OMGHE, vom 6. August 1948 wurde die Information Control Division in Information Services Division umbenannt ${ }^{75}$ - ein kleiner Hinweis auf das veränderte Selbstverstăndnis der Militärregierung.

Der Rückzug der Militärregierung aus der Entnazifizierung führte zur Herabstufung der Entnazifizierungsabteilung vom Status einer eigenständigen Division zu einer Branch der Civil Administration Division. ${ }^{76}$ Die Enforcement Branch der Denazification Division hingegen wurde mit General Order Nr.13 vom 23. August 1948 zur Investigation Division umgebildet. Diese hatte nun im Auftrag des Direktors von OMGHE kriminalistische sowie Kontrollaufgaben durchzufüren und außerdem $\mathrm{Be}$ schwerden und Klagen nachzugehen. ${ }^{77}$

Ubersicht 10: Organisation von OMGHE, 31. Oktober $1948^{78}$

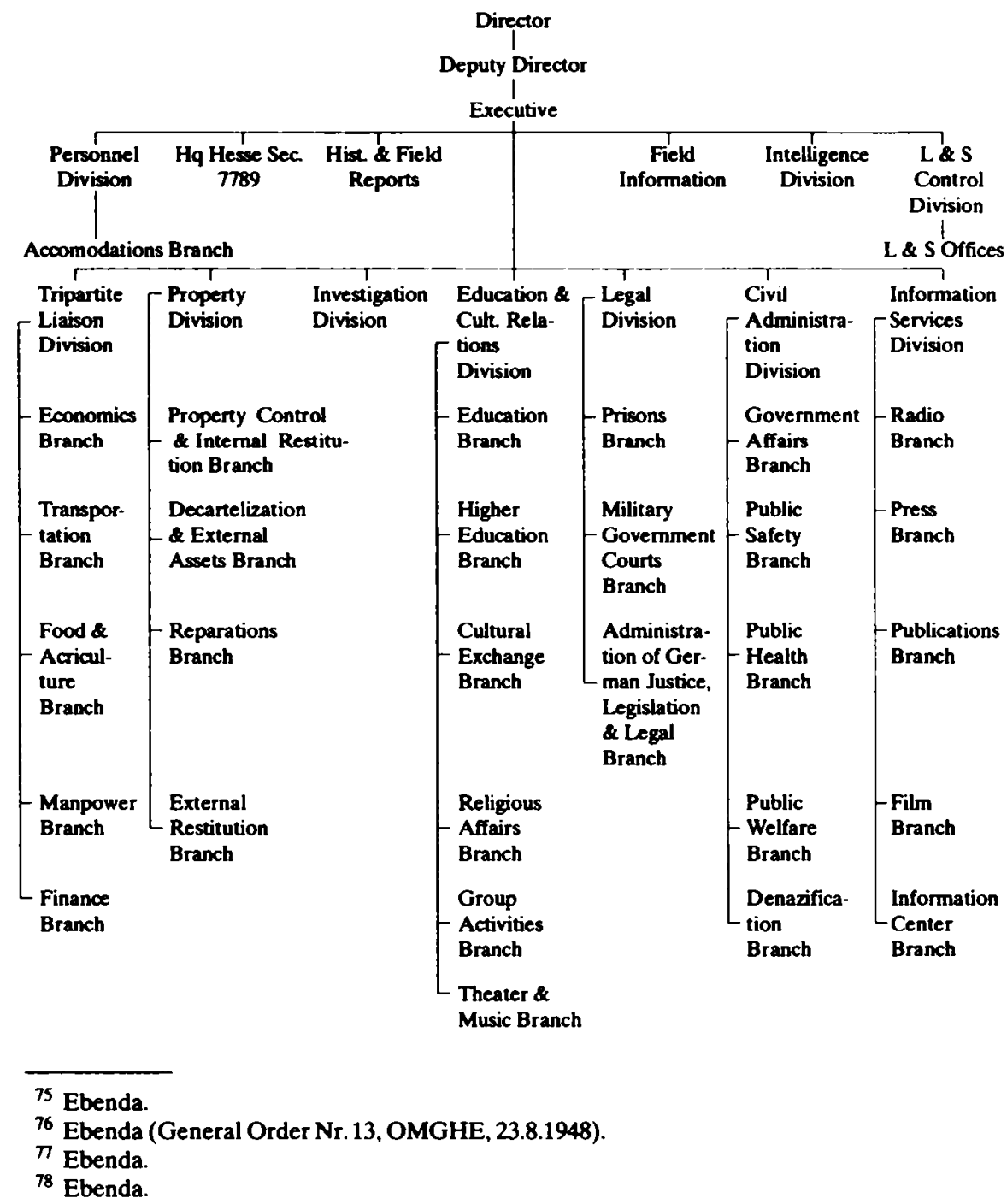


Bis 30. Juni 1949 blieb die Organisationsstruktur unverändert. ${ }^{79} \mathrm{Im}$ Juli wurde die Property Division als Property Branch in die Economic Affairs Division (bisher. Bipartite Liaison Division) eingegliedert. Die Information Services Division wurde aufgelöst. Der Executive Officer wurde durch einen Administrative Assistant ersetzt. ${ }^{80}$

In dieser Form blieb die Organisation der amerikanischen Militärregierung in Hessen bis zu ihrer Auflösung am 20. September 1949 bestehen. $^{81}$ An ihre Stelle trat nach der Charter of the Allied High Commission for Germany vom 6. Juli $1949^{82}$ ein U.S. Land Commissioner, der von Kreis Resident Officers unterstützt wurde. ${ }^{83}$

Ubersicht 11: Organisation von OMGHE, 31.Juli $1949^{20}$

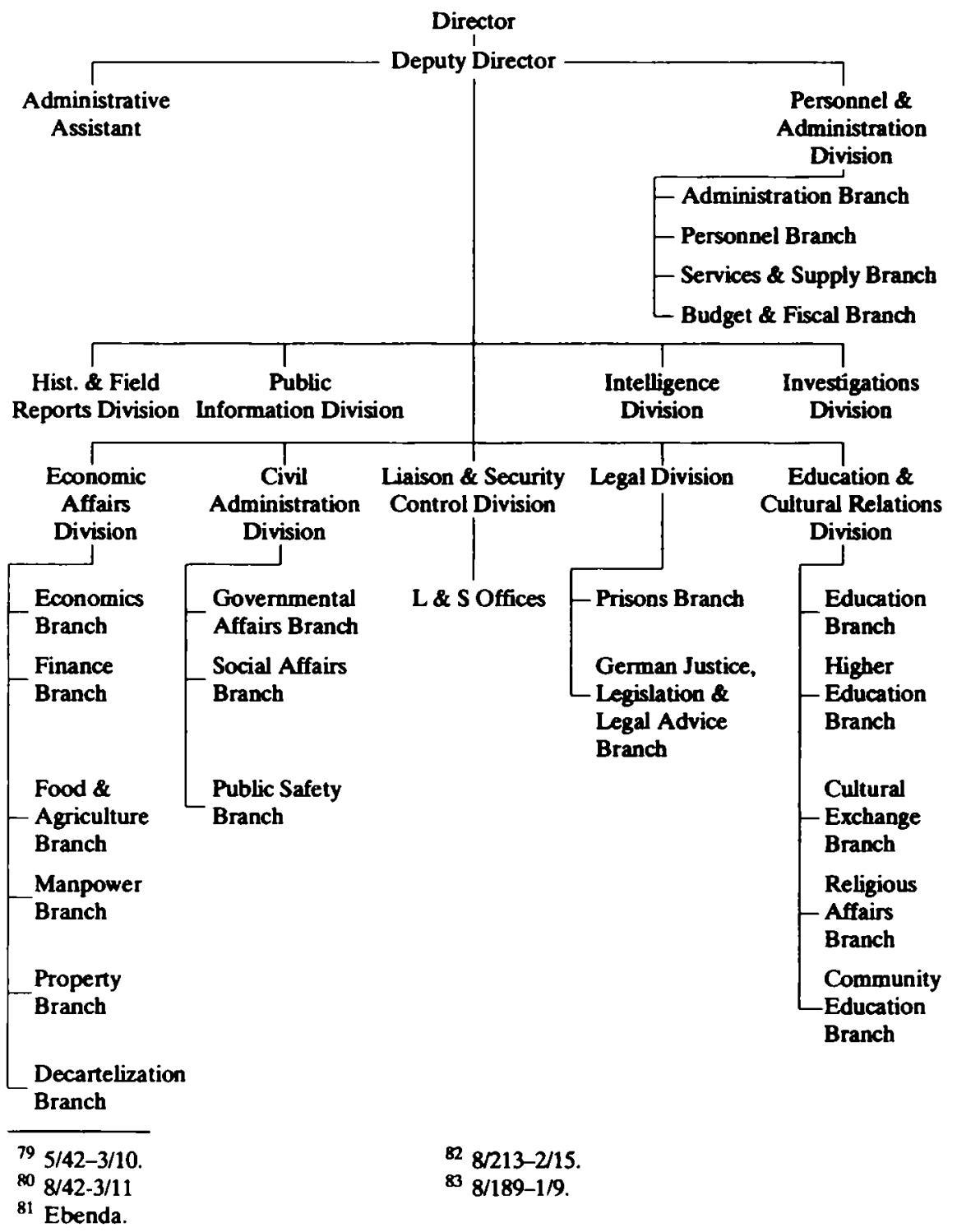




\section{Die lokale und regionale Verwaltung der Militärregierung. Die Detachments}

Ende März, Anfang April 1945 - meist direkt hinter der kämpfenden Truppe - bezogen die meisten Militärregierungs-Detachments ihre Standorte und begannen mit der Arbeit. Diese Arbeit schien schier unerschöpflich, umfaBte sie doch nicht weniger als den Aufbau und die Kontrolle aller Bereiche der öffentlichen Verwaltung. Bis zur Konsolidierung einer Landesmilitärregierung hatten die Detachments in ihren $\mathrm{Zu}$ ständigkeitsbereichen also die alleinige Verantwortung, aber auch die Hauptlast der Arbeit zu tragen.

Je nach Aufgabe und Größe waren die Detachments als E-, F-, G-, H- oder I-Detachments klassifiziert. Die E-Detachments waren die größten und am meisten spezialisierten. Sie waren zuständig für einzelne Länder, Provinzen oder sehr groBe Stadtkreise. Ursprünglich mit einer Personalausstattung von ungefähr 30 Offizieren und circa 50 Soldaten geplant, erreichten einige von ihnen eine wesentlich größere Dimension. Die F-Detachments unterschieden sich nur geringfügig von den E-Detachments. Zuständig für Regierungsbezirke und Stadtkreise, waren sie mit circa 25 Offizieren und etwa doppelt so viel Soldaten ausgestattet. Die G-Detachments waren vorgesehen für Städte, kleinere Regierungsbezirke und gröBere Landkreise. Ihre personelle Ausstattung lag in der Planung bei etwa zwölf Offizieren und circa 20 Mann. Die H- und I-Detachments schließlich waren die kleinsten Einheiten und sollten mit circa vier bis sechs Offizieren und etwa zehn Soldaten für kleinere Städte und kleine und weniger wichtige Landkreise zuständig sein.

Die personelle Ausstattung der Detachments stieg im Sommer 1945 in vielen Fällen auf das Doppelte der Soll-Zahlen an, sank dann aber kontinuierlich, so daB oft die angegebenen Sollstärken unterschritten wurden. Die Spezialisierung des Personals korrespondierte mit der Größe der Detachments. Hatten die E-Detachments für fast alle Aufgaben einen eigenen Spezialisten, so mußte in den I-Detachments häufig ein Offizier vier oder fünf Funktionen übernehmen.

Zunächst hatten die Detachment-Bezeichnungen aus zwei Buchstaben/Zahlen-Kombinationen bestanden, die Größe, Nummer und Truppenzugehörigkeit angaben, (z. B. E1A2 = 1. E-Detachment, Company A, 2nd ECA Regiment). Diese Bezeichnungen wurden zum 15. August 1945 durch Anweisung des Hauptquartiers des 2 nd ECA Regiments so geändert, daß mit dem ersten Buchstaben die Größe des Detachments angegeben und eine laufende Nummer angefügt wurde (z. B. wurde E1 A2 zu E-5).

In Übereinstimmung mit den ursprünglichen Plänen der Amerikaner, zwei Länder auf dem Gebiet des heutigen Hessen zu errichten, hatten für das geplante Land HessenNassau (bestehend aus den Provinzen Kurhessen und Nassau) in Marburg (E-2/ E1G2) und für das Land Hessen in Darmstadt (E-3/E1B2) zunächst zwei LänderDetachments ihre Arbeit aufgenommen. Als Provinzial-Detachments agierten E-4 (E1C2) in Kassel (Provinz Kurhessen), E-5 (E1A2) in Wiesbaden (Provinz Nassau) und E-6 (E1D2) in Frankfurt (SHAEF Enclave). Mit der Schaffung des Landes Greater Hesse durch die Proklamation Nr. 2 war diese Zuständigkeitsverteilung überholt. Das Detachment E-2 in Marburg unter seinem nicht sehr geschickten Kommandanten Col. Charles T. Johnson wurde aufgelöst und seine Ausrüstung dem nun landesweit verantwortlichen Detachment E-5 in Wiesbaden uberstellt. ${ }^{84}$ E-4 ubernahm die Zuständigkeit für den Regierungsbezirk Hessen (später Regierungsbezirk Darmstadt).

${ }^{84}$ OMGHE: Historical Report October 1945 - June 1946. 
Parallel zur Errichtung des Landes Groß-Hessen und mit der Übernahme der Zuständigkeit für den gesamten Landesbereich durch E-5 in Wiesbaden wurde auch die SHAEF Enclave aufgelöst, E-6 zum Stadtkreis-Detachment für Frankfurt erklärt und das Gebiet der SHAEF Enclave dem gleichzeitig für den Regierungsbezirk Wiesbaden zuständigen Detachment E-5 unterstellt. So blieben ab Oktober 1945 noch drei Regierungsbezirks-Detachments bestehen und zwar E-3 in Darmstadt, E-4 in Kassel und $E-5$ in Wiesbaden.

Das Darmstädter Detachment E1B2 war am 16. September 1944 unter seinem kommandierenden Offizier Major Charles J. Gregg bei Rochefort in Frankreich aufgestellt und auf seine Aufgaben vorbereitet worden. Nachdem Ende Oktober 1944 Lt.Col. Clare R. Davis das Kommando über das Detachment übernommen hatte, führte sein Weg über Verdun, Joudreville, St. Avold (Frankreich) und Rumelange nach Esch in Luxemburg (Februar 1945). Als eine der Spearhead Units erreichte E1B2 schlieBlich am 26. März 1945 seinen Einsatzort Darmstadt. ${ }^{85}$

Die Personalstärke von E-3 (E1B2) wird bei der Aufstellung mit elf Offizieren und sechs Mann angegeben. Im Sommer und Herbst 1945 uberschritt das Detachment seine Sollstärke teilweise erheblich und erreichte Zahlen von weit über 70 Offizieren und mehr als das Doppelte an Soldaten. Von Ende des Jahres 1945 bis zur Auflösung der Regierungsbezirks-Detachments Anfang Juni 1946 pendelte die Zahl des Personals, überstieg aber nie die Größenordnung von 10-15 Offizieren und 20-25 Mann. ${ }^{86}$ Die kommandierenden Offiziere von E-3 (E1B2) waren Lt.Col. Clare R. Davis, Lt.Col. William T. Burt (ab November 1945), Lt.Col. R. W. Copeland (ab Januar 1946) und Lt.Col. William R. Swarm (ab Ende Februar 1946).

Das Regionaldetachment E1C2, das am 18. April 1945 an seinem Einsatzort Kassel eingetroffen war, hatte vorher dem Military Government Training Center der 9. USArmee angehört und dort sowohl Amerikaner als auch Briten auf ihre Aufgaben im Rahmen der Militärregierung vorbereitet. ${ }^{87}$

Die 41 Offiziere und 48 Soldaten, die von Tirlemont (Belgien) über Lintfort und Bielefeld nach Kassel gekommen waren, unterstanden der 9. US-Armee (ab Mai 1945 der 7. US-Armee) und waren zuständig für den Regierungsbezirk Kassel und als Provinzial-Detachment gleichzeitig für die Provinz Kurhessen und die hessischen Landkreise Alsfeld, Lauterbach und Gießen. ${ }^{83}$ Der kommandierende Offizier von E-4 (E1C2) war von April 1945 bis zur Auflösung des Detachments im Juni 1946 Lt.Col. Arthur Skarry.

Für das Berichtswesen der Regierungsbezirks-Detachments sollen zwei Beispiele genügen. Bis zur Übernahme der landesweiten Verantwortlichkeit durch E-5 im Oktober 1945 waren die Detachments den jeweiligen G-5-Abteilungen der entsprechenden Armee gegenüber berichtspflichtig, danach gegenüber E-5. Die Berichte folgen mit geringen Abweichungen einem Raster und werden mit abnehmenden Kompetenzen der Regierungsbezirks-Detachments weiter standardisiert.

\footnotetext{
$858 / 190-2 / 8$.

$86 / 188-3 / 3$ und $8 / 190-2 / 8$.

$878 / 187-2 / 3$.

${ }^{88}$ Ebenda.
} 
Ubersicht 12: Raster eines Berichts des Detachments E-4 (E1C2) vom Spätfrühjahr $1945^{89}$

1. Military Government Staffs

2. Civil Administration

3. Political

4. Press and Information Services

5. Public Safety

6. Courts

7. Education

8. Fiscal

9. Property Control

10. Monuments, Fine Arts and Archives

11. Natural Resource

12. Agriculture, Fisheries and Forestry

13. Trade and Industry

14. Labor

15. Civilian Supply

16. Public Works and Utilities

17. Public Health and Welfare

18. Displaced Persons and Refugees

Ubersicht 13: Raster eines Berichts des Detachments E-3 (E1B2), Frühjahr $1946^{\circ 0}$

1. Summary of the situation

2. Special Branch

3. Civil Administration

4. Political Intelligence and Activities

5. Public Safety

6. Legal

7. Property Control

Da den Detachments in der ersten Phase der Militärregierung die Hauptlast der Arbeit zufiel, soll die folgende Aufstellung Aufschluß uber Standorte und Zuständigkeitsbereiche der im Sommer 1945 lokalisierbaren Detachments auf dem Gebiet des späteren GroB-Hessen geben. Zur Vereinfachung werden die Detachmentbezeichnungen, die bis 15 . August 1945 galten, in Klammern angefuhrt. Der Standort wird in Großbuchstaben angegeben, dann folgt der Zuständigkeitsbereich.

Ubersicht 14: Regionale, lokale und funktionale Detachments in Hessen, Sommer 1945

\section{Regionale Detachments}

E-2 (E1G2) MARBURG

Land Hessen-Nassau (Provinzen Kurbessen und Nassau)

E-3 (E1B2) DARMSTADT

E-4 (E1C2) Land Hessen, später RB Hessen, dann RB Darmstadt

$\begin{array}{lll}\text { E-4 } & \text { KASSEL } \\ & \text { Provinz Kurhessen, dann RB Kassel }\end{array}$

E-5 (E1A2) WIESBADEN (vorher NEUSTADT)

$\begin{array}{ll} & \text { Provinz Nassau, RB Wiesbaden, ab Oktober } \\ \text { E-6 (E1D2) } & \text { FRANKFURT/M. } \\ & \text { SHAEF Endave, ab Juli } 1945 \text { SK Frankfurt }\end{array}$

$898 / 11-2 \Omega$.

$908 / 188-3 / 4$. 


\section{Lokale Detachments}

\section{F-12 (F1B2) DARMSTADT}

LK Darmstadt. Für den SK Darmstadt war anfangs H5A9 zuständig, das zwischen dem 19. und 26. Juli mit dem aus Saarbrücken eintreffenden H1 A2 zusammengelegt wurde. Ab 23. August 1945 wurde aus H1A2 und F1B2 das Detachment F-12 SK und LK Darmstadt gebildet. H-64 Ober-Ramstadt (ab

\section{F-13 (F2B2) OFFENBACH}

F-14 (H1C2) KK und LK Offenbach und G-37 Sprendlingen (ab Sept. 1945)

SK und LK Kassel, bis Frulhjahr 1946 I-144 LK Wolfhagen, ab Früjahr 1946

\section{F-15 (F1D2) WIESBADEN \\ G-32 (I10D2) BK Bergstraße \\ G-33 (H1B2) DIEBURG \\ $\begin{array}{lll}\text { G-34 (I11D2) } & \text { FK Dieburg } \\ & & \text { LK Friedberg }\end{array}$}

G-31 (I6B2) BENSHEMM/Fruhjahr 1946 HEPPENHEIM

G-35 (HSD2) GIESSEN

G-36 (H3B2) SK GieBen und H-61 LK GieBen (ab September 1945)

G-36 (H3B2) GROSS-GERAU
LK GroB-Gerau, ab November 1945 zu F-12

G-37 SPRENDLINGEN

ab September 1945 zu F-13

G-38 (I4C2) FRITZLAR

G-39 (F1C2) MAritzlar-Homberg und H-74 LK Ziegenhain (ab November 1945)
MARBURG

SK und LK Marburg

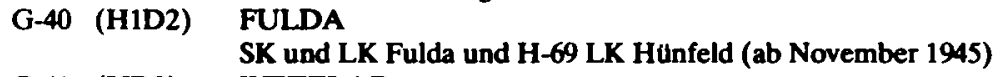

G-41 (I5D2) WETZLAR

G-48 (I6C2) LK Wetzlar

G-48 (I6C2) KORBACH $\quad$ LK Waldeck und H-66 LK Frankenberg sowie I-142 Arolsen (Teil von LK

H-59 (I9D2) Waldeck)

H-60 (18B2) ER Alsfeld, ab November $1945 \mathrm{zu} \mathrm{H}-62$

LK Erbach, ab November zu G-31

H-61 (H5D2) GRÜNBERG

H-62 (I9C2) LAUTERBACH

LK Lauterbach und H-59 LK Alsfeld (ab November 1945)

H-64 (I4B2) OBER-RAMSTADT

LK Darmstadt, ab September zu F-12

H-65 (I8C2) ESCHWEGE

H-66 (I3C2) FRANKENBERG

LK Frankenberg, ab November 1945 zu G-48

H-67 (I1C2) HERSFELD

LK Hersfeld und H-71 LK Rotenburg (ab November 1945) 


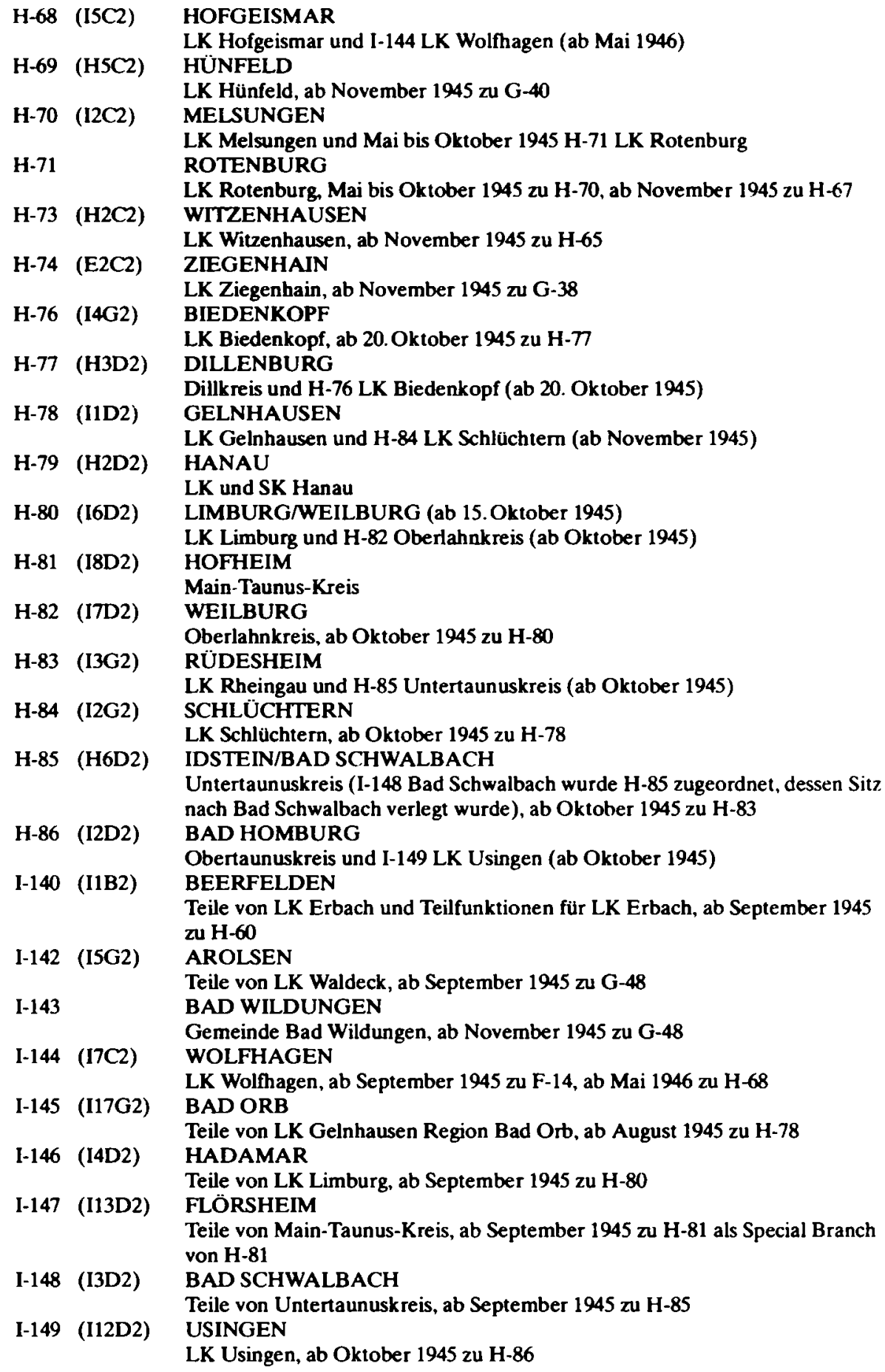


Neben den lokalen Detachments bestanden einige funktionale Detachments, die hier der Vollständigkeit halber angegeben werden.

H-72

ML-1 (SP)

H.87

H-88

\section{KASSEL}

Warehouse Operation, aufgelöst am 11. Januar 1946

KASSEL

Liaison (zugeordnete Verbindungsoffiziere der Alliierten), aufgelöst am 25. Januar 1946

\section{ESSEN}

US Liaison Detachment (Ruhr)

BAD EMS/SULZBACH (ab Mai 1946)

US Liaison Detachment (Saar)

Die beiden letztgenannten Büros waren unter der Zuständigkeit der hessischen Militärregierung am 24. Januar 1946 aufgestellt worden, waren aber der Coal and Mining Section der Economics Division von OMGHE gegenüber verantwortlich. Nachdem die erste Auflösungsanordnung vom 28. Mai 1946 am 10. Juni 1946 widerrufen worden war, wurden sie endgültig zum 20. Oktober 1946 aufgelöst. ${ }^{91}$

\section{Das leitende Personal der Detachments im zeitlichen Querschnitt92}

Übersicht 15: Kommandierende Offiziere der Detachments, OMGHE, Oktober $1945^{93}$

\begin{tabular}{llll} 
Zuständigkeitsbereich & Standort & Kommand. Offizier \\
E-5 & Land Greater Hesse & Wiesbaden & Col. James R. Newman \\
E-3 & RB Hessen & Darmstadt & Lt.Col. Clare R. Davis \\
F-12 & SK/LK Darmstadt & Darmstadt & Lt.Col. L. G. Kelly \\
F-13 & SK/LK Offenbach & Offenbach & Lt.Col. W. A. Snow \\
G-31 & LK Bergstrabe & Bensheim & Maj. W.R. Brown \\
G-32 & LK Biddingen & Büdingen & Capt. L.S. LaPrade \\
G-33 & LK Dieburg & Dieburg & Maj. E. T. Cusick \\
G-34 & LK Friedberg & Friedberg & Maj. R.J. Willard \\
G-35 & SK/LK GieBen & GieBen & Maj. V.L. Ehrenclou \\
G-36 & LK GroB-Gerau & GroB-Gerau & Maj. E.P. Capone \\
H-59 & LK Alsfeld & Alsfeld & Capt. W.P. Burke \\
H-60 & LK Erbach & Erbach & Capt. L. A. Claff \\
H-62 & LK Lauterbach & Lauterbach & Capt. H. Nickelsberg \\
E-4 & RB Kassel & Kassel & Lt.Col. Arthur Skarry \\
F-14 & SK/LK Kassel & Kassel & Maj. R. Bard \\
G-38 & LK Fritzlar-Homberg & Fritzlar & Maj. Robert A. Gish \\
G-39 & SK/LK Marburg & Marburg & Lt.Col. T. A. Brown \\
G-40 & SK/LK Fulda & Fulda & Maj. Charles F. Russe \\
G-48 & LK Waldeck & Korbach & Capt. J.H. Ford \\
H-65 & LK Eschwege & Eschwege & Capt. A. W. Moore \\
H-66 & LK Frankenberg & Frankenberg & Capt. L. E. Morris \\
H-67 & LK Hersfeld & Hersfeld & Capt. G.S. Tredell \\
H-68 & LK Hofgeismar & Hofgeismar & Capt. S.C. Moore \\
H-69 & LK Hünfeld & Hünfeld & Capt. H. H. Liebhafsky \\
H-70 & LK Melsungen & Melsungen & Capt. T. T. Turnbull \\
& & & \\
\hline 91 & $5 / 41-2 / 5$. & & \\
92 & Offensichtliche Tippfehler in den teilweise schwer lesbaren Listen wurden verbessert. \\
93 OMGHE: Historical Report October 1945- June 1946, Appendix I. &
\end{tabular}




$\begin{array}{lll}\text { H-71 } & \text { LK Rotenburg } & \text { Rotenburg } \\ \text { H-73 } & \text { LK Witzenhausen } & \text { Witzenhausen } \\ \text { H-74 } & \text { LK Ziegenhain } & \text { Ziegenhain } \\ \text { I-144 } & \text { LK Wolfhagen } & \text { Wolfhagen } \\ \text { I-143 } & \text { Gm Bad Wildungen } & \text { Bad Wildungen } \\ \text { E-5 } & \text { RB Wiesbaden } & \text { Wiesbaden } \\ \text { E-6 } & \text { SK Frankfurt } & \text { Frankfurt } \\ \text { F-15 } & \text { SK Wiesbaden } & \text { Wiesbaden } \\ \text { G-41 } & \text { LK Wetzlar } & \text { Wetzlar } \\ \text { H-76 } & \text { LK Biedenkopf } & \text { Biedenkopf } \\ \text { H-77 } & \text { LK Dill } & \text { Dillenburg } \\ \text { H-78 } & \text { LK Geinhausen } & \text { Gelnhausen } \\ \text { H-79 } & \text { SK/LK Hanau } & \text { Hanau } \\ \text { H-80 } & \text { LK Limburg } & \text { Limburg } \\ \text { H-81 } & \text { LK Maintaunus } & \text { Hofheim } \\ \text { H-82 } & \text { LK Oberlahn } & \text { Weilburg } \\ \text { H-83 } & \text { LK Rheingau } & \text { Rüdesheim } \\ \text { H-84 } & \text { LK Schluchtem } & \text { Schluchtem } \\ \text { H-85 } & \text { LK Untertaunus } & \text { Bad Schwalbach } \\ \text { H-86 } & \text { LK Obertaunus } & \text { Bad Homburg } \\ \text { l-149 } & \text { LK Usingen } & \text { Usingen }\end{array}$

\author{
Capt. F.W. Hower \\ Capt. W.E. Getman \\ Capt. O.R. Schrimshaw \\ Capt. C.E. Mullin \\ Capt. J. K. Kaup \\ Col. James R. Newman \\ Lt.Col. Robert K. Phelps \\ Lt.Col. F. A. Sansome \\ Lt.Col. Eugene M. Lee \\ Capt. Robert A. Goetcheus \\ Maj. A.T. Kreuger \\ Maj. Matthew F. Chotas \\ Maj. T. Turner \\ Capt. E.F. Duffy \\ Capt. F.H. Percy \\ 1st Lt. B.S. Beaudway \\ Maj. J.G. Gavin \\ Capt. A.H. Harkins \\ Capt. J.I. Cannon \\ Capt. M.L. Campbell \\ Capt. Arsen L. Yakoubian
}

Ubersicht 16: Kommandierende Offiziere der Detachments, OMGHE, 5. Januar $1946^{94}$

Zuständigkeitsbereich

E-5 Greater Hesse

E-5 RB Wiesbaden

E-6 SK Frankfurt

F-15 SK Wiesbaden

G-41 LK Wetzlar

H-77 LK Dill und

LK Biedenkopf

H-78 LK Gelnhausen und LK Schluchtern

H-79 SK/LK Hanau

H-80 LK Limburg und LK Oberlahn

H-81 LK Maintaunus

H-83 LK Rheingau und

LK Untertaunus

H-86 LK Usingen und LK Obertaunus

$\begin{array}{lll}\text { E-4 } & \text { RB Kassel } & \text { Kassel } \\ \text { F-14 } & \text { SK/LK Kassel und } & \text { Kassel } \\ & \text { LK Wolfhagen } & \\ \text { G-38 } & \begin{array}{l}\text { LK Fritzlar-Homberg } \\ \text { und LK Ziegenhain }\end{array} & \text { Fritzlar } \\ \text { G-39 } & \text { SK/LK Marburg } & \begin{array}{l}\text { Marburg } \\ \text { G-40 }\end{array} \\ \text { SK/LK Fulda und } & \text { Fulda }\end{array}$

Hofheim

Rüdesheim
Kommand. Offizier

Col. James R. Newman

Col. James R. Newman

Lt.Col. Francis E. Sheehan

Lt.Col. F. A. Sansome

Capt. William E. Lockwood Maj. D. B. Bernstein

Maj. Matthew F. Chotas

Maj. T. Turner

Capt. E. F. Duffy

Capt. John C. Nelson

Maj. J.G. Gavin

Capt. Arsen L. Yakoubian

Lt.Col. Arthur Skarry

Lt.Col. R. Bard

Capt. G. De Nubla

Maj. Lewis S. Williams

Maj. Charles F. Russe

94 Ebenda. 


\begin{tabular}{|c|c|c|}
\hline G-48 & $\begin{array}{l}\text { LK Waldeck und } \\
\text { LK Frankenberg }\end{array}$ & Korbach \\
\hline H-65 & LK Eschwege & Eschwege \\
\hline H-67 & LK Hersfeld & Hersfeld \\
\hline H-68 & LK Hofgeismar & Hofgeism \\
\hline $\mathrm{H}-72$ & Warehouse Operation & Kassel \\
\hline ML-1 & (SP) Liaison & Kassel \\
\hline-3 & RB Hessen & Darmstadt \\
\hline$F-12$ & $\begin{array}{l}\text { SK/LK Darmstadt } \\
\text { und LK GroB-Gerau }\end{array}$ & 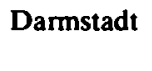 \\
\hline$F-13$ & SK/LK Offenbach & Offenbach \\
\hline G-31 & $\begin{array}{l}\text { LK BergstraBe } \\
\text { und LK Erbach }\end{array}$ & Bensheim \\
\hline G-32 & LK Büdingen & Büdingen \\
\hline G-33 & LK Dieburg & Dieburg \\
\hline G-34 & LK Friedberg & Friedberg \\
\hline G-35 & SK/LK GieBen & GieBen \\
\hline H-6 & $\begin{array}{l}\text { LK Lauterbach } \\
\text { und LK Alsfeld }\end{array}$ & Lauterba \\
\hline
\end{tabular}

Maj. E. J. Dikeman

Capt. W.E. Getman Maj. T. T. Turnbull Capt. S.C. Moore 1st Lt. R. O. Wildish 1st Lt. M. Rogin

Lt.Col. R. W. Copeland L.Col. L.G. Kelly

Capt. E. J. Emerick Maj. A.C. Leggatt

1st Lt. T. A. Norris Capt. J.M. Nutt Maj. R.J. Willard Maj. David M. Easterday Capt. H. Nickelsberg

Ubersicht 17: Kommandierende Offiziere der Detachments, OMGHE, 15. März $.1946^{95}$

Zuständigkeitsbereich

E-5 RB Wiesbaden

E-6 SK Frankfurt

F-15 SK Wiesbaden

G-41 LK Wetzlar

H-77 LK Dill und LK Biedenkopf

H-78 LK Gelnhausen und LK Schlüchtem

H-79 SK/LK Hanau

H-80 LK Oberlahn und LK Limburg

H-81 LK Maintaunus

H-83 LK Rheingau und LK Untertaunus

H-86 LK Obertaunus und LK Usingen

E-4 RB Kassel

F-14 SK/LK Kassel und LK Wolfhagen

G-38 LK Fritzlar-Homberg und LK Ziegenhain

G-39 SK/LK Marburg

G-40 SK/LK Fulda und LK Hünfeld

G.48 LK Waldeck und LK Frankenberg

H-65 LK Eschwege
Standort

Wiesbaden

Frankfurt

Wiesbaden

Wetzlar

Dillenburg

Gelnhausen

Hanau

Weilburg

Hofheim

Rudesheim

Bad Homburg

Kassel

Kassel

Fritzlar

Marburg

Fulda

Korbach

Eschwege
Kommand. Offizier

Col. James R. Newman

Col. Robert K. Phelps

Maj. Matthew F. Chotas

Maj. Michael Baymor

Maj. D. B. Bernstein

Capt. W.F. Johnson

L.Col. T. Turner

Capt. H.L. Edberg

Maj. John C. Nelson

Maj. J. G. Gavin

Capt. Lawrence F. Jones

Lt.Col. Arthur Skarry

Maj. Gerald C. Sola

Capt. G. De Nubla

Maj. Lewis S. Williams

Lt.Col. Charles F. Russe

Capt. H.R. Dichtenmueller

Maj. A.W. Moore

95 Ebenda. 


$\begin{array}{lll}\text { H-67 } & \text { LK Hersfeld } & \text { Hersfeld } \\ \text { H-68 } & \text { LK Hofgeismar } & \text { Hofgeismar } \\ \text { E-3 } & \text { RB Hessen } & \text { Darmstadt } \\ \text { F-12 } & \text { SK/LK Darmstadt } & \text { Darmstadt } \\ & \text { und LK GroB-Gerau } & \\ \text { F-13 } & \text { SK/LK Offenbach } & \text { Offenbach } \\ \text { G-31 } & \text { LK BergstraBe und } & \text { Bensheim } \\ & \text { LK Erbach } & \\ \text { G-32 } & \text { LK Büdingen } & \text { Büdingen } \\ \text { G-33 } & \text { LK Dieburg } & \text { Dieburg } \\ \text { G-34 } & \text { LK Friedberg } & \text { Friedberg } \\ \text { G-35 } & \text { SK/LK GieBen } & \text { GieBen } \\ \text { H-62 } & \text { LK Lauterbach und } & \text { Lauterbach } \\ & \text { LK Alsfeld } & \\ \end{array}$

\author{
Capt. R.W. Godwin \\ Capt. S. B. Borda \\ Lt.Col. William R. Swarm \\ Maj. F.R. Musgrove \\ Capt. C. H. Carter \\ Maj. Lawrence H. Brown \\ Capt. T. A. Norris \\ Capt. John S. Chapin \\ R.J. Williams \\ Maj. David M. Easterday \\ Capt. H. Nickelsberg
}

Die Auflösung einzelner Detachments und die Übernahme der Zuständigkeit durch andere Detachments ist häufig aus folgenden Gründen nicht genau zu datieren:

1. Während der „mobilen Phase“, also bis circa Juli 1945, ergingen Marschbefehle und Aufstellungsbefehle an kleinere Detachments häufig nur mündlich und wurden deshalb nur im Daily Journal notiert. Diese Journals wiederum sind nicht für alle Detachments erhalten geblieben.

2. Offensichtlich sind nicht alle General Orders, mit denen während der "statischen Phase“ Aufstellung, Auflösung oder Zuständigkeitsverlagerung geregelt wurden. überliefert.

3. Der Rekonstruktion aus den vorhandenen Überlieferungen der einzelnen Detachments sind deshalb Grenzen gesetzt, weil es häufig Monate dauerte, bis Anordnungen vorgesetzter Dienststellen ihren Niederschlag im täglichen Schriftverkehr fanden.

4. Die Angaben in den Akten der Militärregierung sind häufig widersprüchlich. ${ }^{*}$

Die Zuständigkeitsverteilung, wie sie sich Anfang 1946 nach verschiedenen Zusammenfassungen und Auflösungen von Detachments ergab, zeigt Übersicht 18. Mit der Konsolidierung der Militärregierung ging ab Ende 1945/Anfang 1946 eine Umbildung der Detachments einher. Nach und nach erhielten sie Liaison and Security Funktionen bis zur endgültigen Umwandlungen aller Detachments in Liaison \& Security Offices (siehe dazu OMGUS-Handbuch, Hessen B.13).

96 Z. B. OMGHE: Historical Report October 1945 - June 1946 und „Combination of Detachments" vom 21.9.1945 in 8/216-2/10. 
Übersicht 18: Zuständigkeitsbereiche der hessischen Regierungsbezirks-Detachments bis zu ihrer Auflösung im Juni 1946 (Stand Ende 1945)

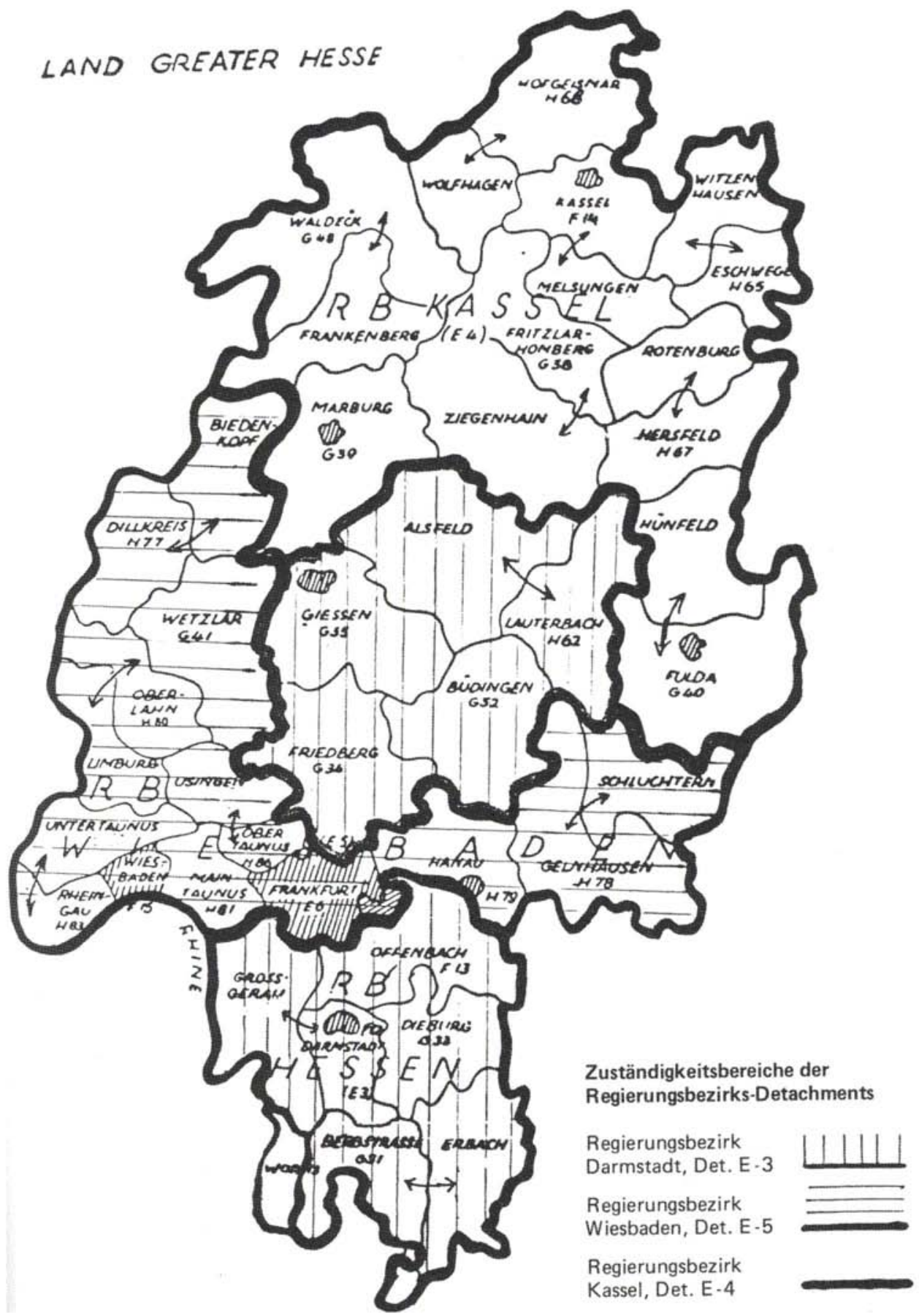




\section{B. Systematische Übersicht Die einzelnen Aufgabenbereiche der Militärregierung für Hessen}

\section{Command and Executive}

\section{a) Aufgaben und Organisation}

Command and Executive war die Stabsabteilung von OMGHE. Sie leitete und kontrollierte die Arbeit der Divisions und Detachments (später Liaison \& Security Offices).

Der Direktor vertrat OMGHE nach außen. Sein Ansprechpartner auf deutscher Seite war der Hessische Ministerpräsident. Innerhalb der amerikanischen Militärregierung für Deutschland war sein Vorgesetzter zunächst der stellvertretende amerikanische Militärgouverneur, seit 15. März 1947 der Militärgouverneur. Beide Ämter hatte nacheinander Lucius D. Clay inne. Weiter repräsentierte der Direktor die Militärregierung bei öffentlichen Anlässen und gegenüber auswärtigen Besuchern. An die hessische Bevölkerung wandte er sich gelegentlich in Radioansprachen.

Gegenüber den anderen Divisions von OMGHE trat vor allem der Executive Officer in Erscheinung. Er verhandelte und korrespondierte auch mit nachgeordneten Stellen von OMGUS und hielt die Verbindung zu den Armee-Einheiten. Ihm zur Seite stand der Assistant Executive Officer. Diese Stellen wurden im Juli 1949 durch einen Administrative Assistant ersetzt.

Der Deputy Director war der Stellvertreter des Direktors (in E-5: Deputy Military Government Officer). Von November 1945 bis September 1947 waren zwei Deputy Directors tätig. Der Deputy Director for Operations war bis zur Bildung einer eigenen Abteilung für Liaison \& Security Control (LSC) im Juni 1946 für die örtlichen Gliederung von OMGHE verantwortlich. Nach Auflösung der SHAEF Enclave war ein zweiter Deputy Director speziell für Frankfurt zuständig. Nachdem der Stelleninhaber zum Bipartite Control Office versetzt worden war, entfiel diese Stelle.

\section{b) Personal}

In Command and Executive waren 1946 unter EinschluB der Funktionsträger acht Amerikaner beschäftigt. Es verwundert nicht, daB es dieser Abteilung gelang, ihren Personalbestand bis Anfang 1949 relativ stabil zu halten - trotz der Personalkürzungen, von denen OMGHE insgesamt betroffen war. Um die Jahreswende 1947/48 waren sogar elf Amerikaner in der Abteilung beschäftigt. Erst in den letzten Monaten wurde das amerikanische Personal reduziert. Am SchluB blieben nur noch der Director und der Deputy Director übrig. Die Zahl der deutschen Mitarbeiter war schon früher geschrumpft. Hatten 1946 vier Deutsche für Command and Executive gearbeitet, so war bereits 1948 nur noch einer tätig.'

Das Amt des Direktors hatte während der gesamten Tătigkeit von OMGHE James $R$. Newman inne, der zuvor als Military Government Officer (MGO) das Detachment

${ }^{1}$ Diese und die Personalzahlen für die folgenden Abteilungen aus 5/41-2/2; 8/179-3/6; OMGHE: Historical Report July-September 1947; 8/215-1/2. 
E-5 bis zu seiner Auflösung am 3. Juni 1946 befehligt hatte. Newman war zunächst Oberstleutnant. Im September/Oktober 1946 wurde er in den Zivilstatus übergeleitet und begegnet uns seit diesem Zeitpunkt in den Quellen als Dr. Newman.

Als Deputy Director war von Juli 1945 bis Januar 1946 Lt.Col. Howard R. Cress, der spätere Leiter der Liaison \& Security Control Division, tätig. Dieses Amt wurde, nachdem es bis Mai 1946 unbesetzt gewesen war, von Francis E. Sheehan bis zum Ende von OMGHE ausgeübt. Sheehan war zuvor Executive Officer gewesen.

Als zweiter stellvertretender Direktor agierte von November 1945 bis Mai 1946 Lt.Col. Charles H. Reed, der später für einige Monate die Public Welfare and Displaced Persons Division leitete. Im Mai 1946 wurde Robert K. Phelps, der frühere Commanding Officer des Detachments E-6 (Frankfurt) zum Deputy Director for Frankfurt ernannt. Dieses Amt übte er bis September 1947 aus (im Oktober 1947 wechselte er zum Bipartite Control Office).

Als Executive Officer war von Juli 1945 bis September 1946 Charles E. Stewart tätig. Vom März bis Mai hatte allerdings der spätere Deputy Director Francis E. Sheehan vorübergehend dieses Amt inne. Nach einem Monat Unterbrechung ubernahm Emest K. Neumann dieses Amt. Nach Wegfall der Position des Executive Officer im Juli 1949 arbeitete Neumann als Chef der Legal Division.

\section{Second Military Government Battalion (Separate) (ab 20. März 1948: Hesse Section, 7780 OMGUS Group)}

\section{a) Aufgaben}

Das Second Military Government Battalion (Separate) - 2 nd MGBat(Sep) - hatte zwei Funktionen:

a) es bildete den militärisch-organisatorischen Rahmen für OMGHE;

b) die dem Battalion direkt unterstellten Einheiten übernahmen für OMGHE alle technischen und verwaltungsmäBigen Aufgaben.

zu a):

2nd MGBat(Sep) wurde am 15. November 1945 fur die innere Verwaltung der hessischen Militärregierung gebildet. Es ubernahm damit die Aufgaben sowie die entsprechenden Einheiten des 2 nd Military Government Regiment, das Bestandteil von ECAD (European Civil Affairs Division) gewesen war und zu der auch Detachment E1A2 (später E-5) und die anderen hessischen Militärregierungsdetachments gehört hatten. 2 nd Military Government Regiment wurde zum 1.Januar 1946 endgultig aufgelöst. ${ }^{2}$ Die Beifügung „(Separate)“ wies darauf hin, daB 2 nd MGBat(Sep) als Sondereinheit nicht Teil der sonstigen militärischen Befehlskette war. ${ }^{3}$

In einem Memorandum vom 11. Februar 1946 hatte J.R. Newman empfohlen, „daß die Bezeichnung Detachment E-5 aufgehoben wird und sein Personal mit dem von 2nd MGBat(Sep) zu einer Einheit, dem Hauptquartier der Militärregierung für GroßHessen, zusammengefaßt wird ... Das gesamte Personal von Detachment E-5 sollte dem 2 nd MGBat(Sep) zur Verwaltung und Versorgung zugeordnet werden. "Zur Begründung führte Newman an, daß „dauernde Verwirrung“ unter seinen Leuten

\footnotetext{
2 8/179-1/6.

3 Vgl. zur Entwicklung der Kommandostruktur OMGUS-Handbuch, Teil Hessen A.
} 
herrschte, da zwei verschiedene Einheiten unter einem (seinem) Kommando zusammengefaßt seien. Die vorgeschlagene Vereinheitlichung könne dies beheben. ${ }^{4}$

Am 3. Juni 1946 kam OMGUS Newmans Vorschlag nach und stellte die gesamte hessische Militärregierung in den organisatorischen Rahmen von 2 nd MGBat(Sep), bei dem es sich also von diesem Zeitpunkt an um die militärische Nomenklatur von OMGHE handelte. Die von Newman beklagte „Verwirrung“ war damit jedoch noch immer nicht beseitigt: In der Frühphase der Militärregierung hätte „eine der Hauptschwierigkeiten darin bestanden, die Vorstellung, daB 2 nd MGBat(Sep) und OMGHE zwei getrennte und unterschiedliche Organisationen seien, auszutreiben“, heißt es im ersten großen Historical Report. ${ }^{5}$

zu b):

2nd MGBat(Sep), wie es als gesonderte Abteilung neben den Divisions in den Organisationsgrafiken von OMGHE auftaucht, bezeichnete die Service-Einheiten, die für die innere Verwaltung von OMGHE zuständig waren. Diese Einheiten erledigten Aufgaben wie

- Fahrbereitschaft

- Gebäude- und Raumpflege

- Post- und Telefonverkehr

- Verpflegung

- Wohnungs- und Büroraumbeschaffung

- Materialbeschaffung

- Freizeiteinrichtungen (Mess)

- Einkaufsmöglichkeiten (PX)

- Arztliche Versorgung

- militärische Personalverwaltung

- Vervielfältigung und Drucke

- Kurierdienst.

Im zweiten Vierteljahr 1947 wurden fast alle dieser Funktionen auf die Military Posts (Militärstandorte) übertragen. Dieser Vorgang war bis zum 1.Juli 1947 abgeschlossen. Beim 2nd MGBat(Sep) verblieben nur noch die Verwaltung von OMGHE-Vermögen, die Koordination der Versorgung, die militärischen Personalakten, die Zentralregistratur, die Reisestelle, die Vervielfältigungsstelle, der Postverkehr und einige kleinere Aufgaben. Vor allem, was den Kurierdienst und die Fahrbereitschaft anlangte, war OMGHE mit der Übernahme der ,housekeeping functions“ durch die Military Posts sehr unzufrieden. ${ }^{6}$ Nach der Überführung von 2 nd MGBat(Sep) in die Hesse Section, 7780 OMGUS Group, übernahm die Personnel Division einen großen Teil der noch bei 2 nd MGBat(Sep) verbliebenen Aufgaben.

\section{b) Organisation}

Bei seiner Bildung am 15. November 1945 bestand 2nd MGBat(Sep) als funktionale Einheit aus folgenden Teilen:

\footnotetext{
4 OMGHE: Historical Report October 1945 - June 1946, Annex.

${ }^{5}$ OMGHE: Historical Report October 1945 - June 1946.

${ }^{6}$ OMGHE: Historical Report April - July 1947.
} 
Ubersicht 19: Organisation von 2 nd Military Government Battalion (Separate), 15. November 1945

- Headquarters

- Headquarters and Service Company, Wiesbaden

- Headquarters and Service Company, Wetzlar

- Company Headquarters. Company ..C“, Mobile Maintenance Platoon, Kirchhain. ${ }^{7}$

Diese in Aufbau und Namensgebung verwirrende Organisation wurde zum Jahresende gestrafft. General Order Nr. 5 vom 29. Dezember 1945 löste das Company Headquarters, Company „C““, auf. Mit General Order Nr.6 vom 31. Dezember 1945 wurde die Headquarters and Service Company in Headquarters Company umbenannt. Die gleiche Order bestimmte die Auflösung des Mobile Maintenance Platoon und die Bildung der Service Company. ${ }^{8}$

Faktisch lag die Leitung der Einheiten in den Händen des Battalion Executive Officer. 2nd MGBat(Sep) hatte damit seit 1946 folgende Struktur: ${ }^{9}$

Übersicht 20: Organisation von 2nd Military Government Battalion (Separate), Januar 1946

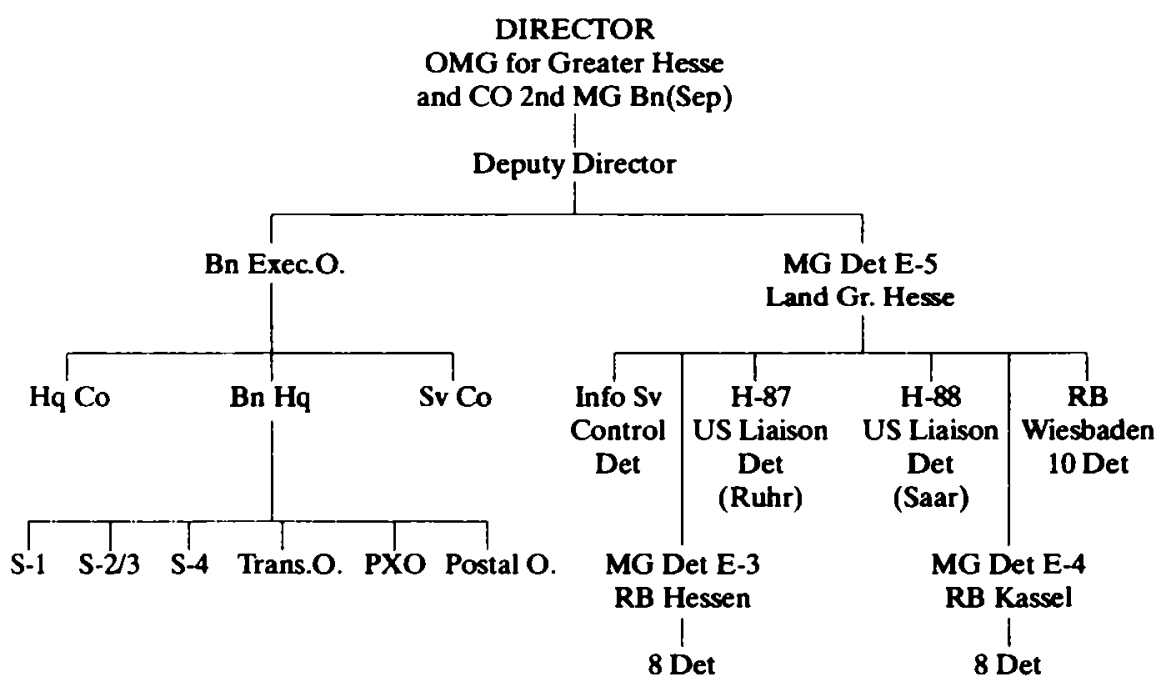

Headquarters Company war für die Personalverwaltung, Disziplinarfälle und Ausbildungsfragen der beim OMGHE-Hauptquartier tätigen Soldaten zuständig. Battalion Headquarters umfaßte Stabs- und Versorgungsabteilungen sowie Poststelle. Service Company war anstelle des aufgelösten Mobile Maintenance Platoon fur die gesamte Fahrbereitschaft von OMGHE zuständig. Sie verlegte ihren Standort am 1.Januar 1946 von Wetzlar nach Oberursel. Im Jahr 1946 verfügte OMGHE über 749 Fahrzeuge. ${ }^{10}$

\footnotetext{
7 OMGHE: Historical Report October 1945 - June 1946.

${ }^{8}$ Ebenda, Annex.

${ }^{9}$ Ebenda.

${ }^{10}$ AG 1945-46/69/10.
} 
Zum 31.Dezember 1945 wurde dem Battalion die 2nd Military Government Medical Group zugeordnet. Das organisatorische Schicksal dieser kleinen Einheit verdeutlicht schlaglichtartig das Durcheinander, das im ersten Vierteljahr 1946 bei der hessischen Militärregierung herrschte. General Order Nr.6 vom 6. Februar 1946 löste die Medical Group auf. Zwei Wochen später, am 19. Februar 1946, wurde mit General Order Nr. 7 diese Auflösung widerrufen. General Order Nr. 8 vom 11. Juni 1946 beendete dann endgültig die Tätigkeit der Group.

In der ersten Jahreshälfte 1946 verschwanden auch die Stabsabteilungen. Die Abteilungen S-2/3 waren wohl von vornherein eher der Form halber ausgewiesen gewesen. Die Personalangelegenheiten (außer fur Soldaten), für die zuvor S-1 zuständig gewesen war, wurden jetzt beim Adjutant des Battalion und in der Personnel Division, OMGHE, geregelt. S-4 (Versorgung/Vermögen) wurde mit der Headquarters Company zusammengelegt.

Eine einschneidende organisatorische Veränderung erbrachte die Abgabe der Verwaltungsaufgaben an die Military Posts im Jahr 1947. Nach dieser im Juli abgeschlossenen Übergabe blieben nur zwei Unterabteilungen bestehen:

- S-4 (Vermögensverwaltung, Versorgung)

- Adjutant's Office (Personalakten und verbliebene Verwaltungsaufgaben). "

Ubersicht 21: Organisation von 2 nd Military Government Battalion (Seperate), 30. Juni 1947

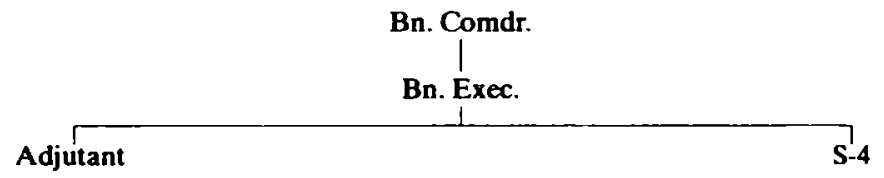

Abgesehen von der Umwandlung der Dienstbezeichnung Executive Officer in Director of Administrative Services im Sommer 1947 blieb diese Organisationsstruktur bis März 1948 bestehen. ${ }^{12}$

OMGUS legte mit General Order Nr.8 vom 8. März 1948 fest, daß die gesamte OMGUSOrganisation ab 30. März 1948 als 7780 OMGUS Group auszuweisen sei. 2nd MGBat(Sep) als Nomenklatur wurde darin zu Hesse Section, 7780 OMGUS Group. Personal und Struktur blieben allerdings unverändert.

Im Organisationsplan von OMGHE trat die Hesse Section, 7780 OMGUS Group als Verwaltungseinheit an die Stelle von 2 nd MGBat(Sep). Die Organisation der Einheit wurde gegenuber 2 nd MGBat(Sep) erneut aufgegliedert in die Bereiche

- Adjutant

- Verwaltung (mit Registratur)

- Service (Versorgung, Vermögensverwaltung)

- Unterstützung der Military Posts.

11 OMGHE: Historical Report April - July 1947, Annex.

$1212 / 168-1 / 3$. 
Ubersicht 22: Organisation von Hesse Section, 7780 OMGUS Group, 31. März $1946^{13}$

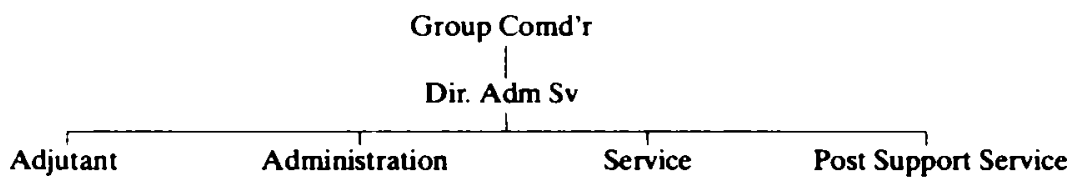

In den Personalverteilungsplänen von OMGHE tauchte jedoch überhaupt nur das Adjutant's Office auf, das seit 30. Juni 1948 gemeinsam mit der Personnel Division gezählt wurde. ${ }^{14}$ Im Frühjahr 1949 entfiel die OMGUS Group auch aus dem Organisationsplan von $\mathrm{OMGHE}$.

\section{c) Personal}

2 nd MGBat(Sep) benötigte sehr viel Personal, um seinen Verwaltungsaufgaben nachzukommen. Im Jahr 1946 arbeiteten 133 Amerikaner im Battalion. Diese Zahl sank leicht zu Beginn des Jahres 1947. Die Übergabe der Verwaltungstätigkeiten an die Military Posts bedeutete einen scharfen Einschnitt: im Herbst 1947 waren nur noch 19 Amerikaner im Battalion. Bis zur Auflösung des 2nd MGBat(Sep) im März 1948 sank diese Zahl auf 15. Diese Mitarbeiter wurden ab Sommer 1948 in der Personnel Division mitgezählt. Im Jahr 1946 beschäftigte das 2nd MGBat(Sep) 710 Deutsche. Diese Zahl stieg bis auf 1050 Anfang des Jahres 1947. Mit der Kompetenzabgabe an die Military Posts entfielen über tausend deutsche Mitarbeiterstellen. Die meisten der Stelleninhaber wurden jedoch von den Military Posts ubernommen.

Bei der Bildung des 2nd MGBat(Sep) am 15. November 1945 ubernahm James R. Newman das Kommando der Einheit. Newman leitete damit kurzzeitig drei verschiedene Institutionen der Militärregierung: OMGHE, Detachment E-5 und 2nd MGBat(Sep). Vom 21. Februar bis 4. März 1946 vertrat ihn kurzzeitig Lt.Col. Charles H. Reed. Die Rechnung von Newman, das Doppelkommando auf Dauer zu behalten, ging nicht auf: am 1.Juni 1946 wurde Lt.Col. Samuel S. Graham Chief des 2nd MGBat(Sep). Graham leitete gleichzeitig die Industry Branch der Economics Division. Er kommandierte das Battalion bis zu dessen Auflösung.

Adjutant der Division war zunächst Capt. Robert A. Goetcheus, der bereits im Dezember 1945 von Major Edwin A. Gehm abgelöst wurde. Auf Gehm folgte Anfang 1947 1st Lt. Robert D. Thayer. Im Sommer 1948, bevor das Adjutant's Office endgultig entfiel, hatte Edward R. Pilli noch kurze Zeit dieses Amt inne.

\section{Personnel Division}

\section{a) Aufgaben}

Bis zum März 1946 wurden die Personalangelegenheiten von OMGHE vom Adjutant und vom Administrative Officer beim OMGHE-Direktor geregelt. Im März begann OMGHE mit dem Aufbau einer eigenständigen Personnel Division (zunächst Personnel Branch genannt), in der nach und nach alle Personalangelegenheiten konzentriert wurden. Dabei oblag der Personnel Division von OMGHE nur die Personalverwal-

\footnotetext{
$135 / 41-2 / 7$.

$145 / 42-3 / 11$.
} 
tung. Die Personalzuweisung war Sache von OMGUS, das auch in Fragen der Verteilung des Personals zwischen den einzelnen Divisions das letzte Wort hatte. Selbst bei der Einstellung der mittleren und höheren Mitarbeiter (Gehaltsstufe CAF-7 und höher) von OMGHE fällte OMGUS letztlich die Entscheidung.

Schwierigkeiten bereitete der Division die Umstellung von militärischem Personal auf Zivilpersonal, bei dem statt militärischer Gesichtspunkte fachliche Qualifikationen für den jeweiligen Arbeitsbereich entscheidend waren. So schrieb die Division im ersten Historical Report: „Es war besonders schwierig, einen Mann, der momentan als Offizier oder als Soldat bereits eine Stelle innehatte, für die er sich jetzt bewarb, davon zu überzeugen, daB er für eine zivile Anstellung wirklich nicht die geringste Qualifikation besaB. " 15 Trotz dieser Schwierigkeiten gelang jedoch in den folgenden Jahren die vollständige „civilianization“ von OMGHE.

Neben den Personalangelegenheiten war die Personnel Division auch für die Budgetverwaltung und -kontrolle von OMGHE zuständig. Diese Aufgabe erhielt die Division eher zufällig, da sie in ihren Reihen einen ausgebildeten Spezialisten für Haushaltsangelegenheiten hatte. Seine Arbeit wurde dadurch erschwert, daB OMGHE seine Mittel von zwei Seiten erhielt: zum einen vom Land Hessen, zum anderen von der amerikanischen Militärregierung.

Zum 30. Juni 1948 erfuhren die Aufgaben der Personnel Division (auch als Personnel \& Administrative Office bezeichnet) eine beträchtliche Erweiterung. Die Division hatte die Verwaltungsaufgaben des im März in die 7780 OMGUS Group liberführten 2nd MGBat(Sep) zu übernehmen. Zwar hatten die Military Posts bereits zum 1. Juli 1947 die Verwaltungsaufgaben (housekeeping functions) ${ }^{16}$ von OMGHE und zum 1. Dezember 1947 die Verwaltung des deutschen OMGHE-Personals übernommen, ${ }^{17}$ doch verblieben die Klassifizierung und Einteilung der Stellen sowie die Budget-Kontrolle bei der Personnel Division. Daneben hatte sie jetzt die Verbindung mit den Military Posts zu ubbernehmen und war zudem fur kleinere technische Dienste und fur die Überwachung der zu dieser Zeit rund 400 deutschen Angestellten von OMGHE zuständig. Schließlich unterhielt sie auch die Poststelle von OMGHE in Wiesbaden. Im zweiten Vierteljahr 1947 richtete die Personnel Division eine Employee and Training Section ein, um den Ausbildungsstand des deutschen und amerikanischen Personals zu verbessern. Im Juni 1947 traf ein zwölfköpfiges Kontingent von Military Government Interns (Praktikanten) ein, die eine Ausbildung bei der Militärregierung absolvierten. ${ }^{18}$ Eine weitere Aufgabe der Personnel Division bestand in der Organisation und Durchführung von Deutschkursen für die Mitarbeiter der Militärregierung. ${ }^{19}$ Auf die Notwendigkeit der Teilnahme an diesen teilweise mehrmonatigen Kursen wurde von OMGUS und vom OMGHE-Direktor immer wieder hingewiesen. ${ }^{20}$

\section{b) Organisation}

Die Personnel Division, die 1946 in der Organisationsgraphik des 2 nd MGBat(Sep) auftauchte und vorubergehend als dessen Teil geführt wurde, war in zahlreiche Bran-

${ }^{15}$ OMGHE: Historical Report October 1945 - June 1946; zur Quellenlage vgl. den Aktenbericht von Gerhard Menk, Staatsarchiv Marburg, vom 5.12.1977, den uns der Verfasser freundlicherweise zur Verfügung stellte.

${ }^{16}$ Chronology, S. 45.

${ }^{17}$ Ebenda und OMGHE: Historical Report July - September 1947.

18 8/215-1/2.

${ }^{19}$ OMGHE: Historical Report April - July 1947.

${ }^{20}$ Z. B. ebenda, auch 8/179-2/1. 
ches aufgegliedert. Neben ihren Branches unterhielt sie noch ein eigenständiges Sekretariat und eine im Chef-Büro angesiedelte Abteilung, die für das militärische Personal zuständig war. Die Personalakten (records) führte allerdings das Adjutant's Office des 2 nd MGBat(Sep).

Die sieben Branches, aus denen sich die Division im Jahr 1947 zusammensetzte, hatten folgende Aufgaben:

- Administration führte die Aktenablage und war für die allgemeinen Angelegenheiten des US-Personals zuständig;

- German Personnel behandelte alle das deutsche Personal betreffenden Vorgänge;

- Placement war für Einstellung und Stellenzuweisung von US-Zivilisten zuständig;

- Budget \& Fiscal verwaltete die OMGHE-Finanzen;

- Classification erarbeitete Stellenbeschreibungen für die OMGHE-Zuweisungen und hatte Planungsaufgaben;

- Payroll war für die Lohnbuchhaltung und für die AbschluBberichte zuständig;

- Employee Relations behandelte Fragen der Arbeitsbeziehungen und des Arbeitsklimas. Die Branch war auch für das Ausbildungsprogramm der Militärregierung verantwortlich.

Ubersicht 23: Organisation der Personnel Division, OMGHE, 30. Juni $1947^{21}$

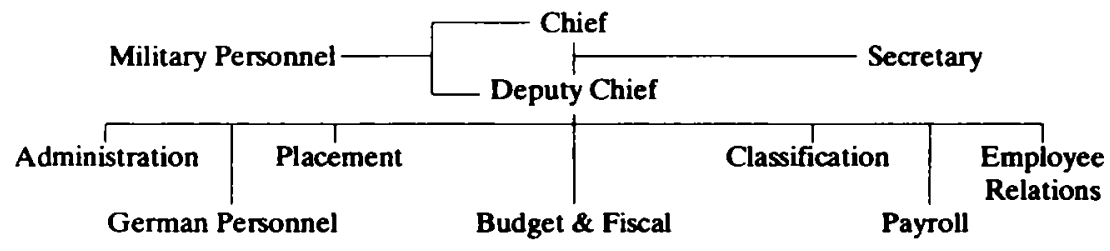

Aufgrund der zum 30. Juni 1948 und teilweise schon vorher vollzogenen Funktionsveränderungen änderte sich auch die Organisationsstruktur der Division. Die für das deutsche Personal zuständige Branch war entfallen, nachdem zum 1. November 1947 die Military Posts diesen Bereich der Personalverwaltung übernommen hatten. Aufgrund der allgemeinen Personalkürzungen fiel die Position des Deputy Chief weg. Die neu geschaffene Administrative Services Branch war für die Verbindung mit den Military Posts zuständig. Employee Relations und Placement wurden zur Employee Utilization Branch zusammengezogen. Administration hatte jetzt zusätzlich einige Aufgaben wahrzunehmen, für die das aufgelöste 2nd MGBat(Sep) zuständig gewesen war: beispielsweise die Ausgabe von PX Cards. Classification, Budget \& Fiscal und Payroll blieben unverändert.

Úbersicht 24: Organisation der Personnel Division, OMGHE, 30. Juni $1948^{22}$

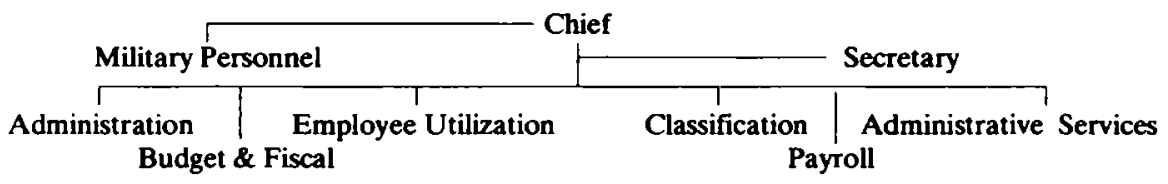

${ }^{21}$ OMGHE: Historical Report April - July 1947.

22 5/41-3/10. 
In den letzten Monaten der OMGHE-Tätigkeit verschmolzen Personnel Division, Administration und Adjutant's Office (früher beim 2nd MGBat(Sep), dann 7780 OMGUS Group) zu einer Einheit. Lediglich in den Organisationsgraphiken fristeten sie eine eigenständige Existenz.

\section{c) Personal}

Nach ihrer Bildung beschäftigte die Division zunächst 16 Amerikaner. Diese Zahl sank bis zum 30. Oktober 1947 auf zehn und zum 15. Mărz 1948 auf sechs ab. 1948 erhöhte sich die nominelle Personalstärke vortbergehend, da jetzt das Personal des Adjutant (7780 OMGUS Group) in der Division mitgezählt wurde. Im Jahr 1949 schrumpfte die Division trotzdem betrăchtlich, so daB ab Mai lediglich fünf Amerikaner in ihr verblieben.

Das deutsche Personal stieg von anfänglich 26 Mitarbeitern auf dem Höhepunkt der Aktivitäten der Division Ende 1946/Anfang 1947 bis auf 46 an, um dann nach Abgabe verschiedener Kompetenzen bis auf zwölf am 15. Mărz 1948 zu sinken. Obwohl die Division einige Aufgaben vom 2 nd MGBat(Sep) ubernommen hatte, beschäftigte sie in der zweiten Jahreshälfte 1948 nur noch zehn Deutsche.

Von der Bildung im März 1946 bis zum Oktober desselben Jahres leitete Melvin W. Kernkamp die Division. An seine Stelle trat dann Robert A. Goetcheus, der bis Januar 1948 im Amt blieb. Ihm folgte sein bisheriger Stellvertreter James R. Haarstad.

\section{Civil Administration Division}

a) Aufgaben

Die Civil Administration Division (CAD) hatte drei Hauptaufgabenbereiche:

a) Sie war für die Zulassung, die Überwachung und das Verbot von Parteien und anderen politischen Organisationen zuständig. Von Verboten waren anfangs vor allem rechtsradikale Gruppen betroffen, die sich schwerpunktmäBig in Nordhessen betätigten. Seit 1947 gerieten die Kommunisten und ihre Aktivitäten zusehends in das Blickfeld und damit unter schärfere Kontrollen. So wurden Parteizeitschriften vorübergehend verboten, und im Jahre 1948 untersagte die Militärregierung die von den Kommunisten angeregten „Volkskongresse“. Ein Verbot jeder weiteren Tätigkeit der Bewegung auf Zonenebene folgte. ${ }^{23}$

b) CAD hielt den Kontakt zu allen Bereichen der deutschen Exekutive und kontrollierte gemeinsam mit der Legal Division die deutsche Gesetzgebung. Besondere Aufmerksamkeit wurde der hessischen Verfassung gewidmet. So setzte sich die Division in einem ausfuhrlichen Memorandum vom 1. November $1946 \mathrm{mit}$ dem hessischen Verfassungsentwurf auseinander, ${ }^{24}$ wobei auffällt, daB sie den später auf Verlangen von OMGUS einer gesonderten Volksabstimmung unterworfenen Artikel 41 (Sozialisierung) noch nicht einmal kritisch kommentierte. Nachdem der hessische Landtag Anfang 1947 seine Gesetzgebungsarbeit aufgenommen hatte, bildeten CAD und Legal Division das Legislative Committee, dem die Chiefs bei-

23 OMGHE: Historical Report 1948. Welche Aufmerksamkeit der VolkskongreB-Bewegung geschenkt wurde, zeigt der Report Nr. 123 der ICD Opinion Surveys vom 25.5.1948: Reactions to the VolkskongreB Petition in Berlin and Darmstadt (IfZ, München).

${ }^{24} 8 / 189-1 / 5$. 
der Divisions angehörten. Seine Funktionen und Aufgaben waren in einem Memorandum des Assistant Executive Officer vom 11. Dezember 1946 beschrieben worden. ${ }^{25}$ Daneben war CAD für die Vertretung von OMGHE in den bizonalen Einrichtungen verantwortlich und kontrollierte die Arbeit der Landesregierung in diesem Rahmen.

c) CAD lberwachte die gesamte deutsche Verwaltungstätigkeit. Dabei nahmen die Amerikaner immer wieder Ansto $B$ am Weiterwirken preuBischer Verwaltungstradition und beklagten die „Obrigkeitshörigkeit“ in den deutschen Behörden. ${ }^{26}$ CAD kümmerte sich auch um den Aufbau der deutschen Verwaltungsgerichtsbarkeit, deren Arbeit sie kontrollierte.

Neben diesen drei Hauptaufgaben betätigte sich CAD gemeinsam mit anderen Divisions in den Bereichen Statistik und Haushaltswesen. 1948 wurden unter der Bezeichnung CAD neben der alten Division die fruher eigenständige Public Safety, Public Health, Public Welfare und etwas später auch Denazification Division vereinigt.

\section{b) Organisation}

1946 war CAD in drei Branches unterteilt:

- Government Organization \& Control Branch

- Civil Service \& Administrative Courts Branch

- Elective \& Civil Organizations Branch. ${ }^{27}$

Diese drei Branches wurden 1947 umbenannt und zwei von ihnen in Sections unterteilt: ${ }^{28}$

Ubersicht 25: Organisation der Civil Administration Division, OMGHE, 30.Juni 1947

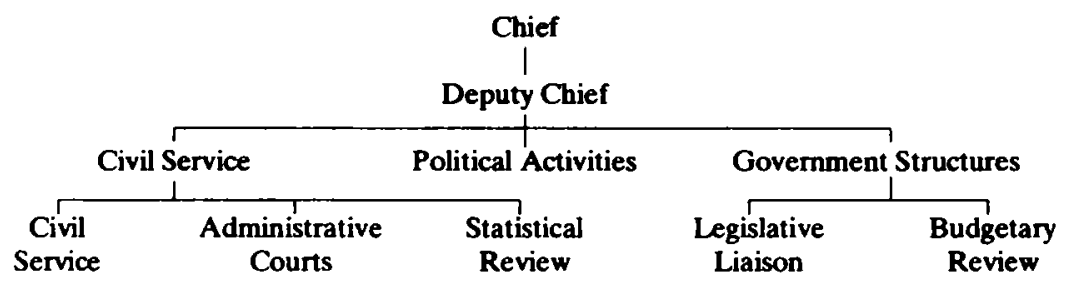

Im Sommer 1947 wurden die Legislative Liaison Branch der Political Activities Branch unterstellt und die Verwaltungsgerichte der Government Structures Branch zugeordnet. ${ }^{29}$ Zum 1. Januar 1948 entfiel Statistical Review, die Aufsicht über die Verwaltungsgerichte ging zur Civil Service Branch zuruck, und die Political Activities wurden dem Deputy Chief direkt unterstellt, ${ }^{30}$ der seit dem 31 .Januar 1948 den Titel Deputy Director hatte..$^{31}$

\footnotetext{
$2517 / 211-3 / 3$.

${ }^{26}$ OMGHE: Historical Report July - September 1947; vgl. auch OMGHE: Historical Report 1948.

27 OMGHE: Historical Report October 1945 - June 1946, Appendix I.

28 OMGHE: Historical Report April - July 1947.

29 Ebenda.

$305 / 41-2 / 17$.

$315 / 41-3 / 10$.
} 
Nachdem J. R. Newman in einem Schreiben an Clay vom 1. Dezember 1947 festgestellt hatte, da $B$,kein Rückgang in den Arbeitsaufgaben der Division“ zu verzeichnen sei, ${ }^{32}$ blieb CAD bei der groBen Umstrukturierung im April 1948 ungeschoren. Mit General Order Nr.5 vom 20. April 1948 wurden dem Chief von CAD vielmehr noch die Bereiche Public Health, Public Safety und Public Welfare and Displaced Persons unterstellt. ${ }^{33}$ Damit waren die früheren Divisions nur noch Branches neben der Governmental Affairs Branch in der neu formierten Division. Zur Verwaltung der vergrößerten Division wurde jetzt eine Administration Branch eingerichtet. ${ }^{34}$ General Order Nr.13 vom 23. August 1948 machte zudem einen Teil der früheren Denazification Division zur Denazification Branch von CAD, so daB die Division seit diesem Zeitpunkt mit folgender Struktur arbeitete: ${ }^{35}$

Ubersicht 26: Organisation der Civil Administration Division, OMGHE, 31. Dezember 1948

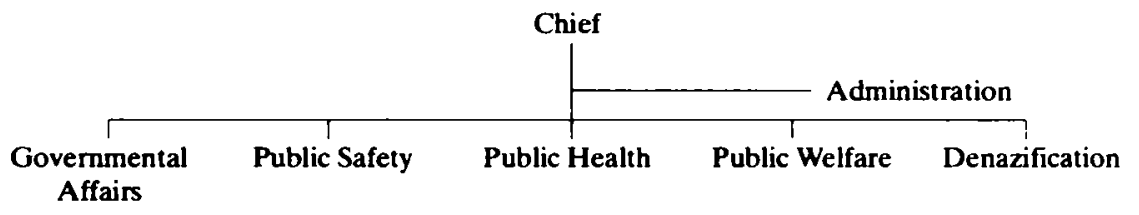

Im Juli 1949 wurden Public Health und Public Welfare zur Social Affairs Branch zusammengefaBt, Denazification entfiel.

\section{c) Personal}

Der spätere Chef von CAD in Hessen, Harold W. Landin, war schon bei der Aufstellung von E-5 am 15.September 1944 in Rochefort mit von der Partie. Er war damals im Rang eines Captain für öffentliche Bauwerke und Kunstschätze zuständig. Am 1. Dezember 1945 wurde er zum Major befördert. In OMGHE wurde ihm dann die Leitung der Civil Administration and Local Government Section übertragen, wo ihn J. R. Newman schon am 1.Dezember 1945 wegen seiner Geschicklichkeit beim Auswählen deutschen Personals für die Landesregierung und für Kommunalverwaltungen besonders belobigte. ${ }^{36}$

Landins Division erhielt von OMGUS am 1.Juni 194613 US-Stellen zugewiesen, ${ }^{37}$ von denen jedoch nur sechs besetzt werden konnten. CAD beschäftigte seit Juli 1946 vier Deutsche, deren Zahl am 1. Januar 1947 auf zehn anstieg. ${ }^{38}$ Bis Mitte 1948 waren so für CAD rund 15 Personen tätig. Durch die Zusammenfassung weiterer Divisions innerhalb von CAD wuchs deren Personal Mitte 1948 auf über 20 amerikanische Mitarbeiter an, um dann kontinuierlich abzusinken.

Harold W. Landin blieb bis Ende 1947 Chef von CAD. Am 15. Dezember 1947 wurde Otto A. Atzert zum kommissarischen Leiter der Division ernannt. (Er war häufig in

\footnotetext{
$328 / 214-1 / 9$.

$338 / 215-1 / 3$.

34 5/41-3/10.

35 Ebenda.

$368 / 216-3 / 4$.

$378 / 179-3 / 8$

$385 / 41-2 / 2$.
} 
den Sendungen von Radio Frankfurt als ,the Voice of Hesse Military Government“ zu hören.) Im Januar 1947 übernahm Dale Noble die Leitung der Division, die er bis zum Ende von OMGHE innehatte.

\section{Economics Division}

a) Aufgaben

Die Aufgaben der Economics Division bestanden in der Kontrolle praktisch aller wirtschaftlichen Aktivitäten in Industrie, Handel, Gewerbe, im Ernährungswesen und in der Landwirtschaft. Diese Überwachung - aber auch Unterstützung - hatte das Ziel, die Arbeit der deutschen Behörden und Organisationen in diesen Bereichen möglichst effektiv zu gestalten. ${ }^{39}$

In der Frühphase der Militärregierung hatten sich die regionalen und lokalen Detachments zusammen mit den Truppeneinheiten direkt dieser Aufgaben angenommen. Gemeinsam mit den Überbleibseln der deutschen Kommunalverwaltung und „besonderen privaten Organisationen“ hatten sie versucht, die öffentlichen Versorgungseinrichtungen wieder in Gang zu setzen. Seit Oktober 1945 waren dann nach und nach die Kontroll- und Überwachungsaufgaben von den lokalen und regionalen Detachments abgezogen und bei E-5 in Wiesbaden zusammengefaBt worden. Dieser ProzeB war Ende 1945 abgeschlossen. „Die Economics Division wuchs von kaum einer Handvoll Mitarbeitern bei Beginn ihrer Tătigkeit zu einer Organisation mit fast fünfzig US-Mitarbeitem an", vermerkte OMGHE im Juni 1946."

Bis Mitte des Jahres 1946 hatte die Economics Division die direkte Kontrolle der wirtschaftlichen Aktivitäten an die inzwischen geschaffenen deutschen Behörden übergeben. Ansprechpartner war jetzt vor allem das am 21 . Oktober 1945 mit Organizational Direction Nr.5, OMGHE, gebildete Ministerium für Wirtschaft und Verkehr. ${ }^{41}$ Das Ministerium für Ernährung und Landwirtschaft ubernahm am 1.Juni 1946 die Kontrolle uber den Konsumgüterbereich (auch Lebensmittel) von OMGHE. Eigene, uber die Kontrolle deutscher Maßnahmen hinausgehende Aktivitäten entfaltete die Division jetzt nur noch in den Bereichen Reparationen, Wiedergutmachung/Ruckfuhrungen, Forschungskontrolle. Daneben unterstutzte sie die Wirtschaftsverwaltung der Armee. ${ }^{42}$

Der Krisenwinter 1946/47 zeigte drastisch, daß die ökonomischen Probleme keineswegs gelöst waren. Die Economics Division bemühte sich daraufhin verstärkt um die wirtschaftlich nutzbare Forschung und konnte nach Ablauf des ersten Vierteljahres 1947 schon über die Existenz von 19 universitären und 45 industriellen Forschungseinrichtungen berichten. ${ }^{43}$ Obwohl sich schon im Laufe des Jahres 1947 Anzeichen wirtschaftlicher Besserung gezeigt hatten, wertete OMGHE im Historical Report von 1948 erst die Währungsreform vom 20 . Juni 1948 als entscheidenden wirtschaftlichen Durchbruch.

${ }^{39}$ 8/81-1/7. Dort auch ein ausführlicher Organisationsplan der Economics Division.

40 OMGHE: Historical Report October 1945 - June 1946.

41 Ebenda und Appendix.

42 OMGHE: Historical Report July - December 1946.

${ }^{43}$ OMGHE: Historical Report January - March 1947. 


\section{b) Organisation und Einzelaufgaben}

Die organisatorische Einteilung der Economics Division vermittelt ein anschauliches Bild über die Aufgaben, die die Division im einzelnen wahrzunehmen hatte. Das Economics Department als Vorläufer der Division war ursprünglich in sechs Branches aufgegliedert, von denen einige in den ersten Monaten quasi selbständig arbeiteten. ${ }^{44}$

Ubersicht 27: Organisation des Economics Department, OMGHE, Oktober 1945

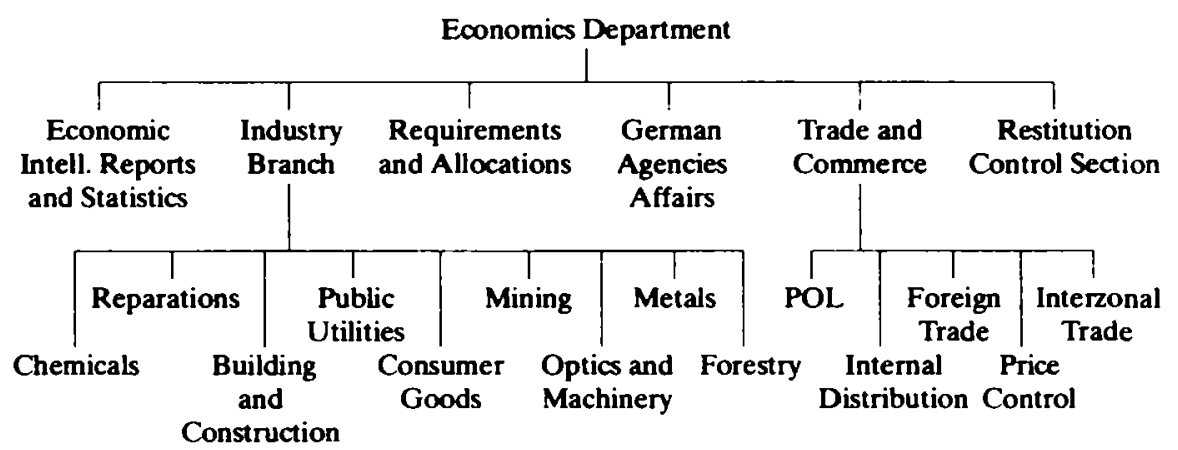

Die Reports and Statistics Branch (Economic Intelligence entfiel 1946) erhob und sammelte Daten über das gesamte hessische Wirtschaftsleben, die von 1947 an in umfangreichen „Statistical Handbooks Economic Activities" zusammengefaBt wurden. ${ }^{45}$ Die Branch wollte mit ihrer Arbeit auch zur Dezentralisierung des deutschen Statistikwesens beitragen. Aufgrund der Neubildung des Landes Hessen traf man hier auf außerordentlich schwierige Arbeitsbedingungen. ${ }^{46}$

Die Industry Branch war für die industrielle Produktion, die öffentlichen Versorgungseinrichtungen und für Reparationsangelegenheiten zuständig. Sie war zunächst in acht, später in neun Sections gegliedert. Die 1946 geschaffene Program Review Section koordinierte die Arbeit der vier für die verschiedenen Sparten der Industrieproduktion zuständigen Sections und überwachte jene Teile der deutschen Produktion, die für die amerikanische Armee notwendig waren. ${ }^{47}$

Die Reparations Section arbeitete auf der Grundlage einer Vereinbarung von OMGHE mit der 7. US-Armee vom 12. Dezember 1945, in der diese die Zuständigkeit für Reparationsfragen an die Militärregierung abgegeben hatte. Gleichfalls im Dezember 1945 war im hessischen Wirtschaftsministerium eine Abteilung für Reparationsangelegenheiten gebildet worden, für deren Überwachung die Reparations Section von OMGHE zuständig war. ${ }^{48}$ Bereits Ende 1946 konnte die Section die Demontage von $94 \%$ der früheren hessischen Luftfahrtindustrie und die Erfassung von 44 für die Reparationen bestimmten Betrieben mit einem Wert von 100 Millionen Reichsmark melden. ${ }^{49}$ Enorme Schwierigkeiten bereitete in der Folgezeit das Perso-

\footnotetext{
44 OMGHE: Historical Report October 1945 - June 1946.

45 In den Anhängen der größeren Historical Reports.

46 OMGHE: Historical Report October 1945 - June 1946.

47 OMGHE: Historical Report July - December 1946.

48 OMGHE: Historical Report October 1945 - June 1946.

${ }^{49}$ Ebenda.
} 
nal- und Transportproblem. Der Historical Report 1948 äußerte den Verdacht, daß die deutschen Arbeitsämter nur die „Lahmen und Blinden“ für Reparationsarbeiten rekrutierten und damit das Reparationsprogramm behindern würden. Auf dem Höhepunkt der Tätigkeit der Reparations Section beaufsichtigten insgesamt sieben amerikanische Ingenieure die Arbeit von circa 3000 deutschen Arbeitskräften. Bereits Ende 1948 kündigte der Historical Report von OMGHE den AbschluB des Reparationsprogramms „,innerhalb der nächsten Monate“ an und vermerkte, daB die Folgen der Reparationen für die hessische Wirtschaft unbedeutend gewesen seien. ${ }^{50}$

Die Requirements Branch ordnete Requisitionen an und kontrollierte die requirierten Güter. Die German Agencies Branch koordinierte die Zusammenarbeit mit deutschen Stellen und beriet die anderen Branches bei dieser Zusammenarbeit. ${ }^{51}$

Die Trade and Commerce Branch, die zunächst aus fünf Sections bestanden hatte, wurde im Januar 1946 in drei Sections umorganisiert, die auch ihre Hauptaufgaben bezeichneten: Importe und Exporte nach und aus Hessen, Handelswesen, Preiskontrolle. Nach Auflösung der Handelsbeschränkungen in den drei Westzonen beschäftigte sich die Import Export Section mit Außenhandelsproblemen. Diese Aufgabe ubernahm im Frühjahr 1948 die hessische Branch der Joint Export Import Agency (JEIA), ${ }^{52}$ deren Leitung der frühere Chef der Trade and Commerce Branch, Julian $A$. Hillman, ubernahm. ${ }^{53}$

Price Control fertigte von September 1945 bis Ende 1946 Zweiwochenberichte an, die über Preisentwicklung, Versorgungslage und Schwarzmarktaktivitäten informierten. In den folgenden Jahren verfaBte sie Monatsberichte. ${ }^{\text {54 }}$

Die POL (Petrol, Oil, Lubricants) Section war für die Beschaffung und Verteilung von Petroleum-Produkten für die Militärregierung zuständig. Gleichzeitig überwachte sie die Neuordnung des deutschen Kraftstoffversorgungswesens mit dem Ziel, wieder „freien Handel“" einzuführen. ${ }^{\text {S5 }}$ Die Zuständigkeit für POL ging 1946 an die Industry Branch uber.

Die Restitution Branch war für die Ruckführung von im Krieg verschleppten Gutern jeder Art zuständig. Nachdem der Bereich Monuments, Fine Arts \& Archives (MFAA) von der Historical Division zu Economics gekommen war, wurde die Branch in zwei Sections aufgeteilt. MFAA war für die Rückfuhrung von Kulturgutern zuständig. Die engagiert tätige Section setzte sich auch fü den Schutz deutscher Kulturguter und die Rückführung ausgelagerten Kulturgutes ein. Sie uberwachte und förderte den Aufbau der Abteilung für kulturelle Angelegenheiten im hessischen Ministerium für Kultus und Unterricht sowie der Landesdenkmalpflege. Diesen Behörden übergab sie nach und nach die ausführenden Tătigkeiten, die sie dann kontrollierte. Bereits im Sommer 1945 waren in Wiesbaden und in Marburg Sammelstellen für verschleppte Kunstwerke (Central Collecting Points for Displaced Arts Objects) eingerichtet worden, die ihre Bestände auch hin und wieder öffentlich zugänglich machten. Im September 1945 entstanden in Frankfurt und in Offenbach Sammelstellen für jüdische Kultgegenstände und Bücher. In Offenbach wurde darüberhinaus im März 1946 ein

\footnotetext{
${ }^{50}$ OMGHE: Historical Report 1948.

51 8/181-1/7.

52 Vgl. Joint Export-Import Agency Charter vom 19.1.1948, in: Germany under occupation, S. 235 - 238.

${ }^{53}$ General Order Nr. 1, OMGHE, 11.1.1948 (5/41-2/17).

$548 / 62-2 / 2$.

55 8/93-1/9.
} 
Archival Depot für nicht-deutsche Bücher und Archive eingerichtet, das für die gesamte US-Zone zuständig war..$^{56}$

In Anlehnung an ein OMGUS-Papier ${ }^{57}$ wurde die Economics Division Anfang 1946 neu strukturiert. Neben den bereits erwähnten Veränderungen auf der Sections-Ebene wurden drei zusätzliche Branches geschaffen: ${ }^{58}$

Ubersicht 28: Organisation der Economics Division, OMGHE, Juni 1946

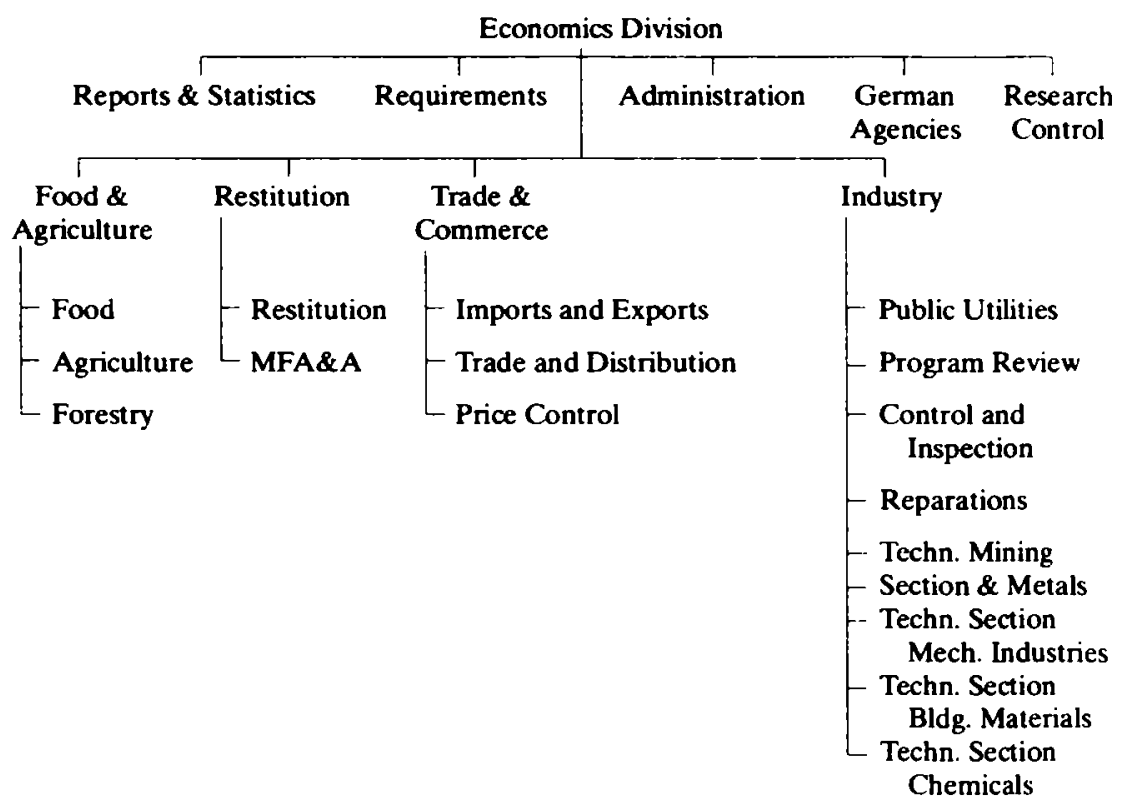

Die Food and Agriculture Branch war bereits im Oktober 1945 auf Landesebene gebildet worden. Gleichzeitig wurde die Verantwortung für Ernährung und Landwirtschaft von den lokalen und regionalen Detachments auf das am 22 . Oktober 1945 mit Organizational Direction Nr.6, OMGHE, gebildete Ministerium für Ernährung und Landwirtschaft übertragen, das von der Branch kontrolliert wurde. Nachdem diese Branch in die Economics Division eingegliedert worden war, erhielt sie auch die Forestry Section zugeordnet, die zunächst zur Industry Branch gehört hatte. Beim Wiederaufbau der Forstverwaltung stand diese Section vor größten Personalproblemen, da die Forstbediensteten unter „Reichsforstmeister“ Hermann Göring zu fast 100 Prozent nationalsozialistisch organisiert gewesen waren. ${ }^{59}$

Die Food Section wurde am 1. Januar 1946 gebildet und war für alle Ernährungsfragen zuständig. Bereits im November 1945 war zudem ein hessisches Landesernährungsamt etabliert worden. Die Food and Agriculture Branch hatte sich zunächst um die Vereinheitlichung des Lebensmittelkartenwesens gekümmert. Food Section kontrollierte

56 OMGHE: Historical Report October 1945 - June 1946.

57 Undatiert in $8 / 81-17$.

58 OMGHE: Historical Report October 1945 - June 1946, Appendix.

59 OMGHE: Historical Report October 1945 - June 1946. 
und überwachte bis Mai 1946 direkt die deutsche Lebensmittelbewirtschaftung und setzte gemeinsam mit den hessischen Behörden die Rationen fest. Im Mai wurden diese Aufgaben auf den Länderrat in Stuttgart übertragen, so daß sich die Section aus der unmittelbaren Kontrolle zurückziehen konnte. Gemeinsam mit der Price Control Section und der Public Safety Division widmete sich die Food Section zunehmend der Schwarzmarktbekämpfung.

Das Lebensmittelkartenwesen war die einzige Möglichkeit, genaue Bevölkerungsdaten zu gewinnen. Diese Daten gab die Section an die anderen Divisions weiter. In den Aufgabenbereich der Section fiel weiter die Beschaffung und Verteilung von Lebensmitteln aus den USA sowie - zusammen mit anderen Abteilungen von OMGHE - die Durchführung von Not- und Hilfsprogrammen (z. B. Child Feeding Program).

Eines der zentralen Probleme der Agriculture Section stellte die Ernteüberwachung dar. Organisatorisch drückte sich das darin aus, daB die Branch neben der Überlastung der Food Section gerade die schwierige Ernteüberwachung immer wieder als Argument für die Anträge auf Personalaufstockung anführte. ${ }^{60}$

Auch die Durchführung der Bodenreform, die in den Zuständigkeitsbereich der Agriculture Section fiel, gab immer wieder Anlaß zur Klage. Zu schleppend schien alles zu gehen: so waren bis Ende 1948 nur 5460 Hektar Land neu verteilt, von denen 3080 Hektar Kleingartenland waren und nur 2380 Hektar für neue Bauernhöfe vorgesehen waren. ${ }^{61}$

Die Research Control Branch arbeitete auf der Grundlage des Kontrollratsgesetzes Nr. 25 (Gesetz zur Regelung und Überwachung der wissenschaftlichen Forschung) vom 29. April 1946. In ihren Aufgabenbereich fielen sowohl die Kontrolle deutscher Forscher und Forschungseinrichtungen als auch die Überprüfung der Anträge auf Wiederaufnahme von Forschungstätigkeiten. Der kleine Stab dieser Branch sah sich häufig gezwungen, Mitarbeiter der Industry Branch zur Unterstutzung heranzuziehen.

Die Anfang 1946 eingeführte Organisationsstruktur der Economics Division blieb bis Mitte 1948 erhalten. Im Sommer 1947 wurde innerhalb der Division die Decartelization Branch gebildet. Bereits am 4. Februar 1947 war - kurz vor der Verkündung des Gesetzes Nr. 56 der Militärregierung (Verbot der ubermäBigen Konzentration deutscher Wirtschaftskraft) - Harry J. Coster als Chef der Research Control zum Beauftragten für alle „Dekartellisierungs-Probleme“ benannt worden. ${ }^{62}$ Die Arbeit dieser wichtigen, wenngleich wenig effektiven ${ }^{63}$ Behörde beschränkte sich in der ersten Zeit auf die Erfassung und teilweise Übernahme der Treuhänderschaft für zu entflechtende Betriebe. Ihre eigentliche Tätigkeit entfaltete diese Abteilung erst innerhalb der Property Division.

General Order Nr.5 vom 20. April 1948 löste die Economics Division auf und überführte ihre Branches in die Bipartite Liaison Division und in die Property Division. ${ }^{64}$

\section{c) Personal}

Die Economics Division gehörte bis zu ihrer Auflösung zu den größten Divisions im Wiesbadener Hauptquartier. Im Juli und August 1946 gehörten ihr 48 Amerikaner und 100 Deutsche an. Etwas größer war nur die Information Control Division. Dieser

\footnotetext{
${ }^{60}$ So am 21.1.1947 (8/123-1/15) und am 6.5.1948 (8/123-1/16).

${ }^{61}$ OMGHE: Historical Report 1948.

62 17/8285/19.

${ }^{63}$ Ebenda.

${ }^{64} 8 / 215-1 / 3$.
} 
Personalstand sank Anfang 1947 auf 31 Amerikaner und 75 Deutsche ab, um sich im Herbst auf 43 amerikanische und 101 deutsche Mitarbeiter zu erhöhen. Damit war Economics vorubergehend personell am besten ausgestattet.

1948 wurde die Division dann wieder von ICD und Legal uberflügelt. Vor ihrer Auflösung im April 1948 hatte sie noch 21 amerikanische Mitarbeiter. Die hohe Personalstärke zeigt, welche große Bedeutung die Amerikaner dem wirtschaftlichen Wiederaufbau - neben dem ideologisch-kulturellen Bereich - zumaBen.

Innerhalb der Division nahm zunächst die Industry Branch mit über der Hälfte der Mitarbeiter eine beherrschende Stellung ein. Nach Einsetzung der bizonalen Wirtschaftsverwaltung entfiel diese Branch jedoch. Übrig blieben vor allem Mitarbeiter im Food and Agriculture Bereich.

Als Chef der Economics Division agierte vom Juli 1945 bis April 1946 Jerome C. White, der in dieser Zeit vom Major zum Lt.Col. aufstieg. An seine Stelle trat der Zivilist Stanley H. Sisson, der die Division bis zu ihrer Auflösung leitete. Sein Stellvertreter war Olav Maseng. Anfang 1947 nahm dieses Amt Carl E. Levan wahr, der dann von Joseph J. Taylor, dem späteren Chef der Economics Branch in der Bizonal Liaison Division, abgelöst wurde.

Die Leitung der wichtigen Food and Agriculture Branch hatte bis zum Sommer 1948 Saul M. Katz. Die Forestry Section dieser Branch leitete William F. Bond, einer der wenigen Mitarbeiter, die von 1945 durchgängig in der hessischen Militärregierung tätig waren. Chef der großen Industry Branch war bis zur Auflösung der Division Samuel S. Graham (bis 1947 Lt.Col.), der nebenher noch das 2 nd MGBat(Sep) kommandierte. Die Trade and Commerce Branch leitete seit Anfang Dezember 1947 Julian A. Hillman, der seit 11.Januar 1948 gleichzeitig Chef der JEIA war. In dieser Branch war George S. Baker Section Chief der wichtigen Price Control Section. Der spätere OMGSTEG (Office of Military Government, Staatliche Erfassungsgesellschaft) Officer furr Hessen, Roy L. Stith, leitete in der Economics Division 1947/48 die Requirements and Allocation Branch. Chef von MFAA war der agile Theodore A. Heinrich, der diese Einheit auch nach ihrer Angliederung an die Property Division weiter leitete.

\section{Bizonal Liaison Division}

\section{a) Aufgaben und Organisation}

General Order Nr.5 vom 20. April 1948 löste die Economics, Transportation, Manpower und die Finance Division auf und bildete aus den aufgelösten Divisions die Bizonal Liaison Division (später Bipartite Liaison Division). Aus der früheren Economics Division wurden die Bereiche Decartelization, Reparations, Restitutions und Monuments, Fine Arts \& Archives in die Property Division uberführt. Die Food and Agriculture Branch wurde als Branch Teil der Bizonal Liaison Division. Die verbliebenen Bereiche bildeten die Economics Branch in der neuen Division. Manpower, Transportation und Finance wurden zu Branches der Bizonal Liaison Division umgebildet und nahmen die bisherigen Aufgaben wahr ${ }^{65}$ - allerdings in reduziertem Umfang: Schon am 1.Dezember 1947 hatte Newman in einem Schreiben an Clay darauf hingewiesen, daB die Finance, die Transportation und die Manpower Division einen

65 8/214-1/9. 
Großteil ihrer Aufgaben erfüllt hätten und personell deutlich reduziert oder, im Fall der Industry Branch, praktisch aufgelöst werden könnten. Nur die Food and Agriculture sowie die Trade and Commerce Branch sollten in ihrem bisherigen Umfang erhalten bleiben. ${ }^{66}$

Mit der anschließenden Umbildung reagierte OMGHE einerseits auf die verminderten Aufgaben der Militärregierung und andererseits auf die Schaffung der Bizone. Die neue Division hatte folgende Organisationsstruktur: ${ }^{67}$

Ubersich 29: Organisation der Bipartite Division, OMGHE, 30.Juni 1948

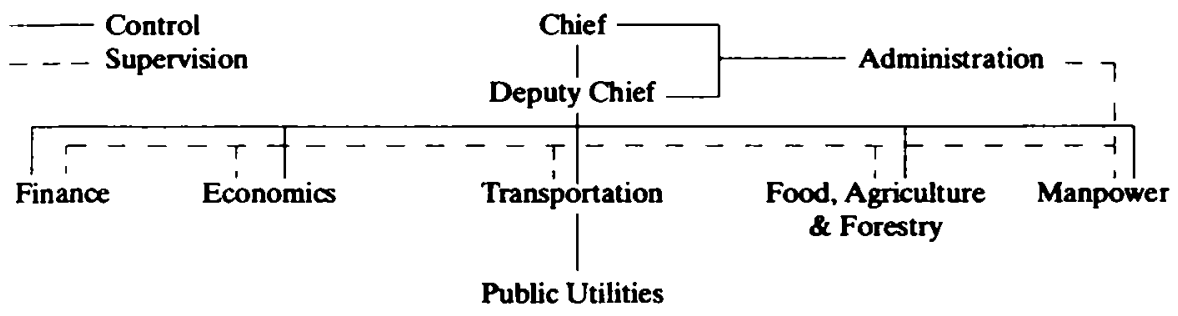

Im Juli 1949 wurde die Bipartite Liaison Division in Economic Affairs Division umbenannt und neu strukturiert. Die Transportation Branch entfiel. Aus der aufgelösten Property Division wurden der Division die Decartelization Branch und die Property Branch angegliedert.

\section{b) Personal}

Am 30. Juni 1948 waren in der neuen Division 22 Amerikaner und 47 Deutsche beschäftigt. Dieser Personalstand verringerte sich zum Jahresende auf zwölf Amerikaner und 39 Deutsche. Im Mai 1949 bestand das amerikanische Personal nur noch aus dem Division Chief, den vier Branch Chiefs und dem Verwaltungsleiter.

General Order Nr. 5 vom 20. April 1948 hatte Stanley H. Sisson, den früheren Chef der Economics Division, zum Leiter der neuen Division ernannt. Zum Chef der Economics Branch ruckte sein fruherer Stellvertreter als Division Chief, Joseph J. Taylor, auf. Zu Branch Chiefs der anderen in der Division eingegliederten Bereiche wurden die früheren Division Chiefs ernannt. Saul M. Katz blieb Leiter der Food and Agriculture Branch. Mit General Order Nr. 10 trat James S. Hathcock am 21 . August 1948 an seine Stelle. General Order Nr.6 vom 22. April 1948 hatte - zusätzlich zu ihren seither ausgeubten Funktionen - Division Chief Stanley H. Sisson zum Economic Adviser und die Branch Chiefs Glenn E. Garrett und Donald S. Spigler zum Manpower bzw. Financial Adviser des OMGHE Director ernannt. General Order Nr. 19 vom 1. Oktober 1948 ernannte Branch Chief James S. Hathoock zum Food and Agriculture Adviser to the Director.

\footnotetext{
66 Ebenda.
}

67 5/41-3/10. 


\section{Education and Religious Affairs Division (ab 1.März 1948: Education and Cultural Relations Division)}

\section{a) Aufgaben}

Folgt man dem langjährigen OMGHE Director James R. Newman, dann lag auf der Education and Religious Affairs Division (ERAD) „die Hauptlast beim Wiederaufbau Deutschlands nach demokratischen Grundsätzen“. Der Erfolg der amerikanischen Besatzungspolitik, so schrieb er 1947 weiter, hänge davon ab, inwieweit es gelänge, die deutsche Jugend zum „democratic way of life“ zu erziehen ${ }^{68}$ So gibt denn auch ein Rechenschaftsbericht eines der ERAD Officer aus dem Jahre 1947 als die erste größere Aktivität in diesem Bereich die Durchführung eines insgesamt 15-stündigen (drei Stunden pro Woche) Seminars über „demokratische Erziehung“ an. Dieses Seminar sei auf „breites öffentliches Interesse " gestoßen und habe dazu gedient, amerikanische Demokratisierungskonzepte und deutsche Reformvorstellungen zu diskutieren. ${ }^{69}$ In allen Kreisdetachments führten die ERA Officers viertägige „Orientation Courses“ für Lehrer durch. Diese Detachment Officers überwachten auch die Wiedereröffnung der Schulen. ${ }^{70}$ In diesem Zusammenhang entwickelten die E-5 Officers im Sommer 1945 ein Formblatt, das das Verfahren zur Wiedereröffnung deutscher Schulen weitgehend standardisierte und das offensichtlich so vorbildlich war, daß es später von den Ländermilitärregierungen in Bayern und Württemberg-Baden übernommen wurde. Von November 1945 bis März 1946 erfolgte die Zusammenfassung der ERA-Aktivitäten auf Landesebene und der Aufbau der entsprechenden OMGHE Division.

Die Division hatte danach folgende Aufgaben und Zielsetzungen:

- Sie überwachte den Aufbau des am 26. Oktober 1945 auf der Grundlage von Organizational Direction Nr.8 gebildeten hessischen Ministeriums für Kultus und Unterricht sowie der Schulverwaltung. Daneben kontrollierte sie die Entnazifizierung der in diesem Bereich Tätigen. Ziel war die „Vereinheitlichung des Schulwesens, die allen Schülern die gleichen Voraussetzungen gibt, um zu aktiven Staatsbürgern in einem demokratischen Staat zu werden" ${ }^{71}$

- Sie überwachte die Berufs- und Erwachsenenbildung. Mit dem Ziel der „Aufklärung der gesamten Bevölkerung" setzte die entsprechende Branch der Division die Einführung von Staatsbürgerkunde gerade in diesen Bereichen durch. Auf ihr Betreiben und mit ihrer Unterstützung bildete sich im August 1946 der Hessische Landesverband für Erwachsenenbildung e. V. Die Adult and Vocational Education Branch führte vom 9. - 11. Dezember 1946 in Wiesbaden die erste Tagung von Berufsschullehrern in der amerikanischen Zone durch. Bildungspolitisch setzte sich die Division für die Einführung des 9. Schuljahres ein und erreichte die Einführung und Anerkennung einer Sonderreifeprüfung an den Volkshochschulen Wiesbaden und Frankfurt. ${ }^{2}$

- Sie sollte in engem Kontakt mit Hochschulverwaltungen, Lehrenden und Studentenschaften beratend und anleitend bei der Neugestaltung der Universitäten tätig werden und deutlich machen, daß „Demokratie mehr als eine äußere Form“ sei.

${ }^{68}$ Brief an Clay vom 1.12.1947 (8/214-1/9).

69 8/215-1/14.

${ }^{70}$ OMGHE: Historical Report 1945 - June 1946.

71 5/41-27.

72 8/215-1/14. 
Das grundlegende Ziel war eine „positive demokratische Veränderung der deutschen Mentalität im Hochschulbereich". ${ }^{73}$

- Gemeinsam mit der Armee überwachte und unterstützte sie die Jugendringe auf Kreis- und auf Landesebene. Ziel war, die deutschen Jugendlichen zu einer aktiven Teilnahme am öffentlichen Leben zu bewegen und eine ,politische und moralische Umorientierung der deutschen Jugend hin zu Demokratie und Frieden" herbeizuführen. ${ }^{74}$ In diesem Zusammenhang und um die Jugendarbeit $\mathrm{zu}$ koordinieren, führte die Division vom 21. - 24. Oktober 1946 in Assmannshausen ein Treffen mit Vertretern der gleichfalls in diesem Bereich tätigen Armeekommandos und Gästen aus Württemberg-Baden, Bayern, Bremen und Österreich durch. ${ }^{75}$

- Im kirchlichen Bereich überwachte die Division die Entnazifizierung und das Verhältnis von Kirche und Staat, wobei auf die Religionsfreiheit besonders geachtet wurde. ${ }^{76}$

Im Laufe des Jahres 1947 setzte sich OMGHE für eine umfassende Schulreform ein. ${ }^{n}$ So verwarfen ihre Vertreter in dem Schulreformplan, den der engagierte Kultusminister Erwin Stein am 1. Oktober 1947 vorgelegt hatte, die Trennung der Schulzüge bereits nach dem vierten Schuljahr (durch Einführung von Latein vom fünften Schuljahr an). Sie forderten mindestens sechs Schuljahre ohne Klassentrennung. ${ }^{78}$

Im Jahr 1948 gewann der Hochschulbereich an Bedeutung. Im Anschluß an eine Studie von zwei führenden Offizieren der Division (Wells/Alexander) über die Situation des hessischen Hochschulwesens erhielt die Division von OMGUS vier zusätzliche University Specialists zugewiesen.

Ende Juni 1948 wurde eine Cultural Exchange Branch eingerichtet. Aufgaben im Bereich des Kulturaustausches hatte zuvor in geringem Umfang die Higher Education Branch wahrgenommen. Diese Verstärkung des Kulturaustausches war Ausdruck einer sich im Jahr 1948 insgesamt verändernden Akzentsetzung in der Arbeit der Division. An die Stelle der gezielten und kontrollierenden EinfluBnahme auf einen beschränkten Personenkreis von Multiplikatoren (z. B. Pfarrer, Lehrer) trat der Versuch, sich durch Filme, Amerikahäuser, Austauschprogramme usw. direkt an die Bevölkerung zu wenden. Dieser Versuch fand seinen offiziellen Ausdruck im Reorientation Program, das OMGHE am 25. August 1947 anordnete. ${ }^{79}$ Dem in diesem Zusammenhang gebildeten „Reorientation Committee“ gehörte der Chef der Division an. Innerhalb der Division wurde die Youth Activities Branch in Group Activities Branch umbenannt und war nun für die Bereiche Frauenarbeit, Jugendaktivitäten, Erwachsenenbildung und audiovisuellen Medieneinsatz zuständig.

Die Branch war damit u. a. verantwortlich für die Villa Lilly Leadership Training School, ${ }^{80}$ die Oberreifenberg Training School und die Community School AudioVisual Aids Program (CSA). ${ }^{81}$ Während sich die ersten beiden Einrichtungen der Jugendarbeit widmeten, führte CSA Filmvorführungen durch und bildete Filmvorführer aus.

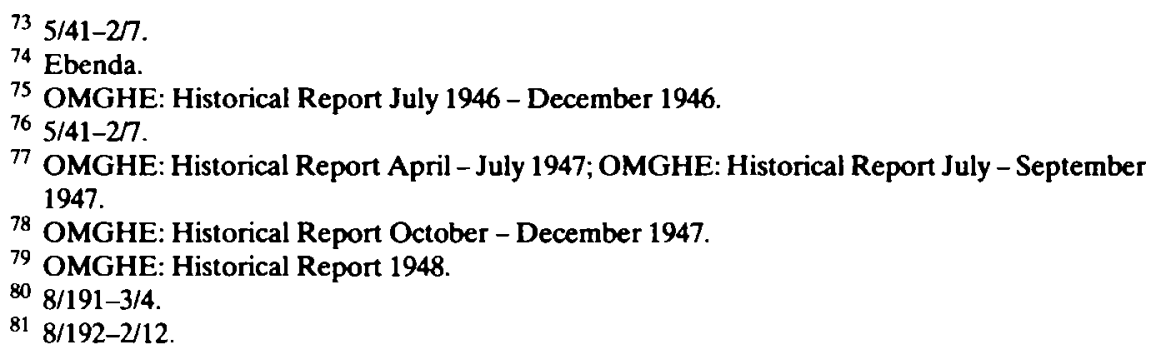


Daneben fielen zu dieser Zeit praktisch alle im Rahmen des Reorientation Program durchgeführten Projekte in die Zuständigkeit der ECR Division, die dabei eng mit anderen Divisions zusammenarbeitete. Diese Projekte, an denen oft Experten aus den USA mitwirkten, sollten dem Erlernen „demokratischen Verhaltens“ auf allen Ebenen und in allen Bevölkerungsgruppen dienen.

Im Laufe des Jahres 1948 wurde eine Verlagerung der Schwerpunkte in der Zielsetzung der Division deutlich: in den Aufgabenbeschreibungen der Branches entfielen im wesentlichen die Demokratisierungsforderungen. ${ }^{82}$ In den Hauptzielsetzungen des Reorientation Program wurde neben den bekannten Forderungen nach Sicherung der Freiheitsrechte des einzelnen und demokratischen Strukturen auf allen Ebenen nun auch „die Schaffung eines Bollwerks gegen die Propagierung totalitärer philosophischer Ideologien" verlangt. ${ }^{83}$

\section{b) Organisation}

Bei ihrer Aufstellung war die Division zunächst in sieben Branches gegliedert. Nachdem die beiden Field Teams General Education (vier Stellen) und Curricular Democratization (eine Stelle) in andere Branches eingegliedert und eine eigenständige Administration Branch eingerichtet worden war, präsentierte sich die Division von Mitte 1947 bis Mitte 1948 in folgender Organisationsstruktur: ${ }^{84}$

Ubersicht 30: Organisation der Education and Religious Affairs Division, OMGHE, 30. Juni 1947

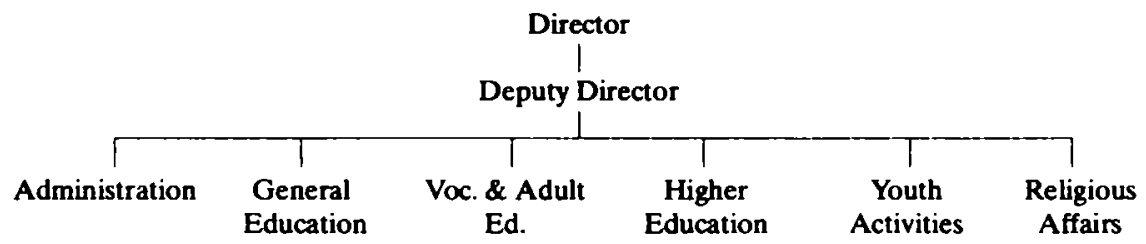

Die von OMGUS am 18. Februar 1948 herausgegebenen General Orders Nr. 6 und Nr. 8 zur organisatorischen Neuordnung des ERA-Bereichs vollzog OMGHE nur teilweise nach. Während die hessische Militärregierung mit General Order Nr.4 vom 1.März $1948^{85}$ die von OMGUS mit General Order Nr. 8 angeordnete Umbenennung der Division in "Education and Cultural Relations Division“ vollzog, folgte sie General Order Nr. 6 nicht. Am 8. März 1948 wies Henry A. Wann, der Direktor der hessischen Division, in einem Schreiben an Newman darauf hin, daß es nicht möglich wäre, "die Veränderung sofort vorzunehmen" ${ }^{86}$ In der Tat verzichtete OMGHE auf Dauer darauf, die Division - wie von OMGUS angeordnet - in vier Branches zu gliedern, sondern hielt an den bestehenden fünf Branches mit einer gesonderten Administration Section fest. Als hessische Besonderheit bestand eine Higher Education Branch fort. Die Youth Activities Branch wurde allerdings in Group Activities umbenannt und erhielt die bereits erwähnten zusätzlichen Aufgaben.

\footnotetext{
$825 / 41-3 / 10$.

$838 / 191-3 / 4$.

$845 / 41-27$.

85 5/41-2/17.

$868 / 215-1 / 14$.
} 
Anfang 1948 wurden die Aufgaben der Vocational and Adult Education Branch auf die Group Activities und die Education Branch verteilt. Ende 1948 bildete OMGHE einer OMGUS-Anweisung entsprechend eine Cultural Exchange Branch. Am 6. August $1948 \mathrm{kam}$ noch die Theatre and Music Branch, die zuvor zur Information Control Division gehört hatte, dazu, ${ }^{87}$ so daB die Division in den letzten Monaten der Besatzungszeit folgende Struktur hatte: ${ }^{88}$

Übersicht 31: Organisation der Education and Cultural Relations Division, OMGHE, 31. Dezember 1948

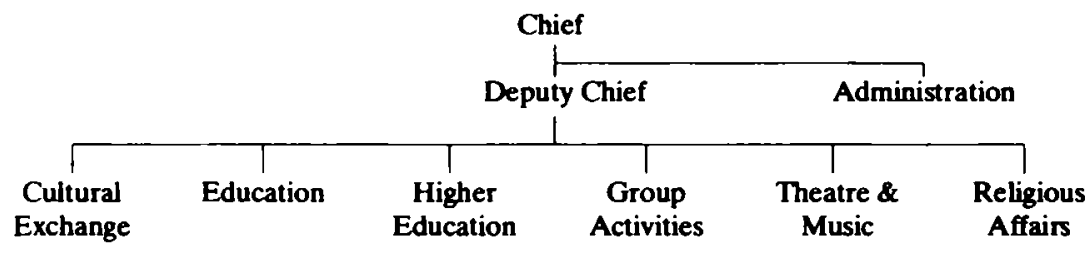

\section{c) Personal}

Der Personalstand der ERAD zeichnete sich durch eine bemerkenswerte Konstanz aus. Von 1946 bis Anfang 1949 arbeiteten in der Regel 21 Amerikaner in der Division. Nachdem sich Newman gegenüber OMGUS am 1. Dezember 1947 für eine Beibehaltung der Division ausgesprochen hatte, ${ }^{89}$ uberstand sie die groBe Veränderung des Jahres 1948 praktisch unbeschadet. Dadurch wurde sie zu einer der gröBten Divisions von OMGHE, was auch ihrer gestiegenen Bedeutung im Rahmen der von der amerikanischen Regierung 1947/48 forcierten ideologischen Arbeit Rechnung trug.

Die Zahl der deutschen Mitarbeiter erhöhte sich von sieben am 1.Juli 1946 auf 40 am 31. Dezember 1948 - eine einmalige Entwicklung innerhalb von OMGHE. Weiter war in dieser Division der Anteil der promovierten Mitarbeiter, die entweder aus dem schulischen oder aus dem universitären Bereich kamen, wesentlich höher als in anderen Divisions.

Schon bei der Aufstellung von E-5 am 15. September 1944 war der Major und spätere Lt.Col. James F. Bursch für Erziehung und Religion zuständig gewesen. Nach Bildung der ERA Division übernahm er bis Mai 1946 deren Leitung. Zu diesem Zeitpunkt rỉckte Vaughn De Long, der vorher als Deputy Chief tătig gewesen war, zum Chief auf. Auf De Long folgte Dr. Henry A. Wann, der von Januar 1947 bis August 1948 die Division leitete. Vom August 1948 bis zur Auflösung übernahm dann wieder Vaughn R. De Long dieses Amt. Deputy Chief der Division wurde als Nachfolger von De Long Dr. Leroy Vogel, dessen Position aber schon Anfang 1949 entfiel.

Die Higher Education Branch leitete seit ihrer Bildung Dr. James Hartshorne. An seine Stelle trat 1947 Dr. Howard P. Becker, der Ende 1948 von Dr. Franz Montgomery abgelöst wurde. Chief der Religious Affairs Branch war zunächst Dumont F. Kenny. Dieses Amt übernahm Ende 1948 George F. Donovan. Seit Sommer 1945 beschäftigte sich Erwin Dingman mit der Berufs- und Erwachsenenbildung. Nachdem ERAD die Arbeit aufgenommen hatte, wurde er zum Chief der entsprechenden Branch ernannt. Diese Stelle ubbernahm im Frühjahr 1948 kurzzeitig Dr. Howard Oxley, der nach Auf-

\footnotetext{
87 General Order Nr.8, OMGHE, 6.8.1948 (5/41-3/10).

88 5/41-3/10.

$898 / 214-1 / 9$.
} 
lösung der Vocational and Adult Education Branch Chief der neu gebildeten Group Activities Branch wurde. Nach den Umstrukturierungen im Frühjahr 1948 übernahm Dr. Eugene R. Fair die Leitung der Education Branch. Zur gleichen Zeit wurde Dr. Marie Schnieders Chefin der Cultural Exchange Branch. Die Leitung der Theatre and Music Branch, die im Sommer 1947 von ICD zu ECRD verlegt worden war, hatte zunächst William P. Dubensky inne, der schon bei ICD in diesem Bereich tätig gewesen war. Diese Funktion übte 1949 dann Dr. Everett B. Heim aus.

\section{Finance Division}

\section{a) Aufgaben}

Die Finance Division war für Währungsfragen, die öffentlichen Haushalte und die Überwachung des deutschen Geld- und Bankwesens zuständig. Zunächst hatte jedes Detachment über einen für Finanzfragen zuständigen Offizier verfügt. Diese unterstanden der Aufsicht der Regierungsbezirks-Detachments.

Nach der Bildung eines deutschen Finanzministeriums in Hessen durch Organizational Direction Nr.4, OMGHE, vom 20. Oktober 1945, das nun die deutsche Finanzverwaltung kontrollierte, konnte die Finance Division über das Ministerium ihre Aufsichtsfunktionen wahrnehmen. Im November 1945 entfiel die Zuständigkeit der Landund Stadtkreisdetachments für finanzielle Fragen. Die Regionaldetachments zogen sich im Dezember 1945 aus dem Finanzbereich zurück, die Verantwortung lag seit dieser Zeit allein bei der Division. Diese versuchte, das deutsche Finanz- und Bankwesen gründlich zu entnazifizieren. Insgesamt wurden uber 3600 Beschäftigte in diesem Bereich zunächst relegiert. ${ }^{90}$

Im November 1945 gab die Division der Currency Section, Finance Division, OMGUSZ, einen Überblick über die Bestände der hessischen Reichsbankfilialen. ${ }^{91}$ Die Bankguthaben führender Nationalsozialisten wurden gesperrt. ${ }^{92}$ Anfang 1947 überwachte die Division die Neustrukturierung des hessischen Finanzministeriums, dessen auf fünf angewachsene Abteilungen bis zum 1. Mai 1947 zusammen in einem Gebäude untergebracht werden sollten.93

Die Eingriffe der Finance Division in die deutsche Politik verringerten sich in den folgenden Monaten. Einsprüche gegen den hessischen Haushaltsplan, die sie Ende 1947 vortrug, konnten ,informell“ geklärt werden. ${ }^{94}$ Die Division drang allerdings häufig darauf, daß die Landesregierung ihre Haushalte rechtzeitig vor Beginn der Rechnungsjahre einbrachte. Auseinandersetzungen zwischen dem hessischen Finanzministerium und OMGHE ergaben sich dadurch, daB das Ministerium nach Ansicht von OMGHE zu hohe Prokopfzahlen für die Belastung Hessens durch Besatzungskosten in die öffentliche Diskussion einbrachte.

Am 27. Februar 1948 übergab OMGHE die Aufsicht über gesperrte Bankguthaben an die Landesregierung. Die Einsetzung einer deutschen Wirtschaftsverwaltung für die Bizone (MG Proclamation Nr.7, Bizonal Economic Administration, vom 9. Februar 1948) und die Errichtung der Bank deutscher Länder zum 1.März 1948 (MG Law

90 OMGHE: Historical Report October 1945 - June 1946.

91 OMGHE: Historical Report 1945.

92 OMGHE: Historical Report October 1945 - June 1946.

${ }^{93}$ OMGHE: Historical Report January - March 1947.

94 OMGHE: Historical Report October - December 1947. 
Nr. 60 vom 1. März 1948) verringerten die Aufgaben der Finance Division weiter. ${ }^{45}$ Mit General Order Nr. 5 vom 20. April 1948 wurde sie endgültig aufgelöst. Mit stark reduziertem Personal nahm fortan die Finance Branch in der Bizonal Liaison Division und danach in der Economic Affairs Division die verbleibenden Aufgaben wahr. ${ }^{\text {* }}$ Die Branch beobachtete vor allem die Folgen der auf Grundlage der Militärregierungsgesetze Nr. 61 - 65 am 20. Juni 1948 durchgeführten Währungsreform. ${ }^{97}$

\section{b) Organisation}

Die Organisationsstruktur, die sich nach Konstituierung der Division herausgebildet hatte, blieb bis zu ihrer Auflösung am 20. April 1948 erhalten. Eine der drei Branches beschäftigte sich mit den öffentlichen Haushalten, die zweite verfolgte Mißachtungen von Anweisungen der Militärregierung. Die dritte Branch war für das Bank- und Kreditwesen, Versicherungen und Währungsfragen zuständig. Sie kontrollierte außerdem die gesperten Bankguthaben.

Im Juni 1947 hatte die Finance Division folgende Struktur. ${ }^{83}$

Ubersicht 32: Organisation der Finance Division, OMGHE, 30. Juni 1947

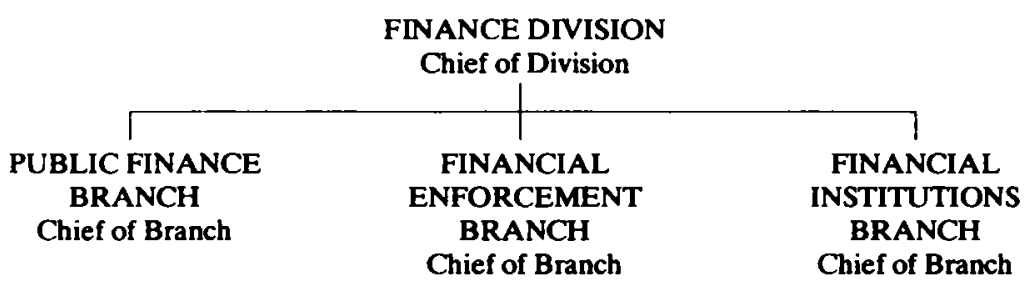

c) Personal

Die Finance Division gehörte zu den kleineren Divisions von OMGHE. 1946 verfügte sie über sieben amerikanische und elf deutsche Mitarbeiter. Während die Zahl der deutschen Mitarbeiter bis Ende 1947 konstant blieb, wurde das amerikanische Personal um drei Positionen auf vier verringert. Vor Auflösung der Division waren nur noch zwei Amerikaner bei ihr beschäftigt, die 1948 in der Finance Branch der Bizonal Liaison Division weiterarbeiteten. Im Frühjahr 1949 entfiel eine Position, so daß am Ende der Tätigkeit von OMGHE nur noch ein Finanzberater für diesen Bereich zuständig war.

Leiter der Division war zunächst Major John D. C. Baldwin, der am 20. Oktober 1947 von seinem bisherigen Stellvertreter Donald S. Spigler abgelöst wurde. Spigler wurde am 22. April 1948 zum Finanzberater des Direktors von OMGHE ernannt. Als sein Stellvertreter arbeitete bis zum 20. September 1948 Paul J. Zeller. Nachdem Zeller seinen Dienst beendet hatte, übernahm John A. Grey seine Aufgabe.

95 OMGHE: Historical Report 1948.

86 8/215-1/3.

97 OMGHE: Historical Report 1948.

98 OMGHE: Historical Report April - July 1947. 


\section{Foreign Liaison Division (auch French Liaison)}

Ende 1947 wurden die noch bei OMGHE tätigen ausländischen Verbindungsoffiziere, für die zunächst der Executive Officer von OMGHE zuständig gewesen war, ${ }^{99}$ in einer eigenen Division zusammengefaBt. ${ }^{100} \mathrm{Da}$ es sich zu diesem Zeitpunkt nur noch um drei Franzosen handelte, wurde die Division in den OMGHE-Aufstellungen zum Teil auch als French Liaison geführt. In den ersten beiden Jahren der Militärregierung waren zahlreiche ausländische Verbindungsoffiziere in Hessen tätig gewesen. Ihre Aufgabe bestand vor allem in der Ruckführung und Betreuung von Landsleuten, die sich als Soldaten oder DPs in Deutschland befanden. Die Verbindungsoffiziere waren entweder amerikanischen Truppenteilen oder Detachments der Militärregierung zugeordnet (attached). ${ }^{101}$ Eine eigenständige Foreign Liaison Division bestand nur bis März/April 1948.

\section{Information Control Division (ab 6. August 1948: Information Services Division)}

\section{a) Aufgaben}

„Die Aufgaben von Information Control umfassen die Bereiche Presse, Zeitschriften, Bücher, Filme, Musik und Radio. Information Control beschäftigt sich also mit dem gesamten Medienbereich", schrieb die Division im ersten Historical Report von OMGHE. ${ }^{102}$

Zunächst hatte das 6871st District Information Services Control Command (DISCC) diese Aufgaben wahrgenommen. 6871st DISCC war im März 1945 als Teil der Psychological Warfare Division von SHAEF gebildet worden und agierte als Einheit der 7. US Armee unter deren Kommando im Western Military District. Nach der Auflösung dieses District mit General Order Nr.1, USFET, vom 2. Januar $1946^{103}$ beschränkte sich 6871st DISCC auf Wurttemberg-Baden. Die Information Control-Aufgaben in Hessen ubernahm das 6840th Theater Information Services Control Command (TISCC), das seinen Sitz in Bad Nauheim hatte. Zu diesem Zeitpunkt hatte die Einheit in Hessen acht Zeitungen, 26 Verleger und fünf Theater zu kontrollieren. 6840th TISCC wurde allerdings bereits im Januar 1946 wieder aufgelöst.

General Order Nr.4, 2nd MGBat(Sep), vom 25. Januar 1946 ordnete die Aufstellung des Information Services Control Detachment in OMGHE an. ${ }^{104}$ Die neugeschaffene Abteilung verlegte ihren Sitz von Bad Nauheim und SchloB Georgenborn, wo TISCC vorübergehend seinen Sitz gehabt hatte, nach Wiesbaden. Das Information Services Control Detachment wurde jedoch mit General Order Nr.5, 2 nd MGBat(Sep), vom 4. Februar 1946 wieder aufgelöst. ${ }^{105}$

99 OMGHE: Historical Report October 1945 - June 1946, Appendix.

100 OMGHE: Historical Report October - December 1947.

101 Aufstellungen in 8/179-1/15; für E-4 (Kassel) Aufstellungen in 8/180-1/2, 8/180-1/3 bzw. 8/187-3/9.

102 OMGHE: Historical Report October 1945 - June 1946; vgl. zum folgenden auch Hurwitz: Die Stunde Null, S. $120 \mathrm{ff}$.

103 12/226-1/1-11.

104 OMGHE: Historical Report October 1945 - June 1946.

105 Ebenda, Appendix. 
An seine Stelle trat die am 28. Februar 1946 auf Grundlage einer USFET-Anweisung vom gleichen Tag gebildete Information Control Division (ICD), OMGHE. ${ }^{106}$ Die Division wies starke personelle Kontinuitäten zu ihren drei Vorläuferorganisationen auf. Der Organisationsaufbau war am 1.Juni 1946 vollendet. ${ }^{107}$ Allerdings waren noch im Juli 1946 zwei Chief of Branch-Stellen und eine Deputy Chief of Branch-Stelle unbesetzt. ${ }^{108}$

Schwierigkeiten bereitete die Tatsache, daB die Aufgaben, die 6840th Theater Information Services Control Command auf Zonenebene wahrgenommen hatte, nunmehr neu verteilt werden mußten. Der Information Control Division von OMGHE fiel bei dieser Zuständigkeitsverteilung im Frühjahr 1946 die Aufgabe zu, hauptverantwortlich für den Aufbau und die Kontrolle der DANA (Deutsche Allgemeine Nachrichten-Agentur) zu zeichnen. ${ }^{109}$ Die anderen zonalen Aufgaben, ausschlieBlich derjenigen, die ausdrucklich in die Zuständigkeit der entsprechenden Abteilungen der Länder-Militärregierungen gegeben worden waren, ubernahm im Laufe des Sommers 1946 Information Control Division, OMGUS, und Information Control Service, OMGUS. ${ }^{110}$

Innerhalb von ICD, OMGHE, waren die Aufgaben auf einzelne Branches verteilt. So beschäftigte sich die Press Branch mit dem Zeitungswesen. Ihre erste Aufgabe, die sie noch im Rahmen der Vorläuferorganisationen von ICD in Angriff genommen hatte, bestand in der Auswahl geeigneter Lizenztrăger für die in den gröBeren Stădten zu gründenden Zeitungen. Als erste hessische Zeitung erhielt die „Frankfurter Rundschau“ am 31. Juli 1945 ihre Lizenz und durfte ab 1 . August 1945 zunächst mit 500000 Exemplaren erscheinen.

Die Branch fuhrte beständige Klage uber chronischen Personalmangel und Unterbezahlung des vorhandenen Personals. ${ }^{111}$ Auseinandersetzungen zwischen deutschen Stellen und OMGHE ergaben sich des ofteren, weil die Amerikaner die Zeitungen gegenuber Angriffen aus Parteien und Behörden grundsătzlich in Schutz nahmen. ${ }^{112}$ In den Aufgabenbereich der Press Branch fiel auch - wie oben erwähnt - der Aufbau und die Kontrolle von DANA in Bad Nauheim. Diese Stadt wurde deshalb zum Sitz der Agentur, weil das nationalsozialistische Propagandaministerium seine Ausrustung dorthin ausgelagert hatte.

Alle anderen Druckerzeugnisse außer den Zeitungen, die eine Sonderstellung einnahmen, fielen in den Zustăndigkeitsbereich der Publications and Periodicals Branch, die daruberhinaus auch die kommerziellen Leihbibliotheken kontrollierte.

Die Radio Branch beschäftigte sich mit Radio Frankfurt, dem zunächst einzigen Rundfunksender in Hessen. ${ }^{113}$ Ein Kontrolloffizier war bei allen Ausstrahlungen dieses Senders zugegen. ${ }^{114}$ Der technische Betrieb des Senders ging im Laufe des Jahres 1946 in deutsche Hände über. Schwierigkeiten ergaben sich mit der Übernahme der inhaltlichen Regie. Schon für 1947 vorgesehen, wurde sie erst im Herbst 1948 Wirk-

\footnotetext{
106 8/216-3/8 und OMGHE: Historical Report October 1945 - June 1946.

107 OMGHE: Historical Report October 1945 - June 1946.

108 8/155-1/13.

$1098 / 216-3 / 8$.

110 OMGHE: Historical Report October 1945 - June 1946.

111 Ebenda; 8/160-2/78/179-3/4.

112 OMGHE: Historical Report 1948.

113 OMGHE: Historical Report October 1945 - June 1946.

$1148 / 215-2 / 11$.
} 
lichkeit, nachdem der Landtag am 22. September 1948 ein Rundfunkgesetz verabschiedet hatte, dem OMGHE zustimmte. ${ }^{115}$

Die Film, Theater and Music Branch war an verschiedenen Stellen untergebracht. Während die Bereiche Theater and Music von Wiesbaden aus betreut wurden, residierte die Film-Abteilung in den ehemaligen UFA-Räumen in Frankfurt. Die Branch kontrollierte die öffentlichen Aufführungen in ihrem Bereich, bei denen im übrigen nur Künstler auftreten durften, die zuvor ein entsprechendes Lizenzierungsverfahren der Branch hinter sich gebracht hatten. ${ }^{16}$ Die Film Section verwaltete dariberhinaus bis zum 1.Februar 1948 den gesamten Filmverleih. Seit Februar 1948 übernahmen nach und nach kommerzielle Agenturen den Verleih. ${ }^{117}$ In ihren Äußerungen gegenüber den anderen OMGHE-Stellen machte sich die Branch oft zum Anwalt der Künstler, indem sie beispielsweise auf deren schlechte Versorgungssituation hinwies. ${ }^{118}$

Die Intelligence Branch von ICD hatte zunächst eine doppelte Aufgabe: einmal sollte sie Informationen über Verhalten und Ansichten der Deutschen - besonders gegenüber den Amerikanern - sammeln. Zum anderen hatte sie die Lizenzbewerber für den künstlerischen und publizistischen Bereich auf ihre demokratische Tauglichkeit zu überprufen.

Mit einer Direktive vom 22. Januar 1946 erhielt die Branch zusätzlich den Aufgabenbereich „Political Intelligence“, d. h. die Überwachung politischer Aktivitäten auf deutscher Seite, zugeteilt. Die Zentrale in Wiesbaden sammelte die Berichte der acht Außenstellen und gab wöchentlich einen acht- bis zehntausend Worte umfassenden Bericht, den „German Public Opinion Review“, heraus. Allerdings begann schon im Februar 1946 die Bildung einer eigenständigen Organisationseinheit für diesen $\mathrm{Be}$ reich (später Intelligence Division). ${ }^{119}$

Auf zonaler Ebene entsprach dieser Entwicklung die Einrichtung einer eigenen, wissenschaftlichen Standards verpflichteten „Survey Unit“ als Unterabteilung der Intelligence Branch der Information Control Division, OMGUS. Das Hauptquartier dieser Einheit war aus organisatorischen Gründen der Landesmilitärregierung Hessen angegliedert; sie unterhielt aber auch in Bayern, Württemberg-Baden und Berlin lokale Büros mit festem Personal. ${ }^{120}$

Die Aktivităten der Intelligence Branch verlagerten sich im Laufe der Zeit von der politischen Aufklärung in den Forschungsbereich. Diese Entwicklung fand schließlich in der Umbenennung der Branch in Research Branch im Sommer 1947 ihren Ausdruck. ${ }^{121}$ Die Aufgabenbereiche Lizenzvergabe und -kontrolle blieben jedoch erhalten. ${ }^{122}$ Anfang 1948 wurde die Branch erneut in Media Investigation Unit umbenannt. ${ }^{123}$

Die Exhibition and US Information Centers Branch war zuständig für die Aufsicht über die seit Anfang 1946 in folgenden Städten eröffneten Information Centers (Amerika-Häuser):

115 OMGHE: Historical Report 1948.

116 OMGHE: Historical Report October 1945 - June 1946.

$1178 / 215-2 / 11$.

118 OMGHE: Historical Report April - July 1947.

119 OMGHE: Historical Report October 1945 - June 1946.

${ }^{120}$ Ebenda.

121 OMGHE: Historical Report April - July 1947.

$1228 / 215-2 / 11$.

${ }^{123} 8 / 176-1 / 26$ 
Ubersicht 33: US Information Centers (Amerika-Häuser) in Hessen, 1946-1948

$\begin{array}{ll}\text { Marburg } & \text { (eröffnet am 26. Februar 1946) } \\ \text { Frankfurt } & \text { (eröffnet am 22. Mai 1946) } \\ \text { Kassel } & \text { (eröffnet am 19. September 1946) } \\ \text { Darmstadt } & \text { (eröffnet am 16. Januar 1947) } \\ \text { Gießen } & \text { (eröffnet am 15. Mai 1947) } \\ \text { Wiesbaden } & \text { (eröffnet am 8. Juli 1947) }^{124} \\ \text { Fulda } & \text { (eröffnet im Juli 1948) }^{125}\end{array}$

Die Centers führten Sprachkurse und öffentliche Veranstaltungen durch. Weiter verfügten sie über Leihbibliotheken. Für die Auswahl des Buchbestandes war ebenfalls die Exhibition and US Information Centers Branch zuständig. Darüber hinaus richtete die Branch in $38 \mathrm{Kreisstädten} \mathrm{Büchereien} \mathrm{ein.} \mathrm{Die} \mathrm{sieben} \mathrm{Centers} \mathrm{verfügten} \mathrm{über}$ ein „Bookmobile“, das kleinere Gemeinden mit Lektüre versorgte. Im Jahr 1948 verzeichneten die Centers 991000 Besucher und 497000 Buchausleihen. ${ }^{126}$

Mit General Order Nr.9 vom 6. August 1948 erhielt ICD die neue Bezeichnung Information Services Division (ISD). ${ }^{127}$ Diese Umbenennung der Division brachte den Funktionswandel und die Umorientierung der amerikanischen Politik zum Ausdruck: von der „Kontrolle“ zum „Service“. So spielte ISD eine wichtige Rolle im Reorientation Program. ${ }^{128}$ Der Division Chief war Mitglied im Reorientation Committee von OMGHE.

Ein pessimistisches Fazit der ICD/ISD-Arbeit in Hessen zog der Leiter der Media Investigation Unit, Max $\mathbf{H}$. Kimental, in einem Brief an seinen Division Chief vom 13. Juni 1949. ${ }^{129} \mathrm{Zwar}$ seien ,die deutschen Medien in diesem Land [ . . ] alles in allem [...] nicht gegen die Demokratie“, die Meinungsführer schienen aber noch nicht „über ausreichende Selbstsicherheit, genug Ruckgrat und vielleicht auch Mut zu verfugen, um die Angriffe der noch immer bestehenden nationalsozialistischen Phalanx niederzuschlagen".

\section{b) Organisation}

ICD war in sieben Branches gegliedert, die ihrerseits wieder in Sections unterteilt waren. Im Juni 1946 war die Division wie folgt organisiert (Übersicht 34). ${ }^{130}$

Bis zum Juni 1947 wurden folgende Veränderungen vorgenommen:

- Die Plans, Operations and Liaison Branch, die für Stabsaufgaben und Außenkontakte zuständig war, wurde als Branch aufgelöst. Der Organisationsbereich wurde dem Chief direkt zugeordnet.

- Für die US Information Centers, deren Zahl sich in diesem Zeitraum von zwei auf fünf erhöhte, war jetzt nicht mehr nur eine Section der Publications Branch, sondern eine eigene Branch zuständig, die gleichzeitig mit dem Ausstellungswesen betraut war.

- Die frühere Intelligence Branch wurde zur Research Branch.

\footnotetext{
${ }^{124}$ Chronology, S. 16, 21, 28, 36, 43, 46.

125 OMGHE: Historical Report 1948.

126 Ebenda.

$1275 / 41-3 / 10$.

${ }^{128}$ Vgl. OMGUS-Hb,Teil Hessen B.7: Education and Religious Affairs Division.

$1298 / 176-1 / 26$.

130 OMGHE: Historical Report October 1945 - June 1946.
} 
Ubersicht 34: Organisation der Information Control Division, OMGHE, Juni 1946

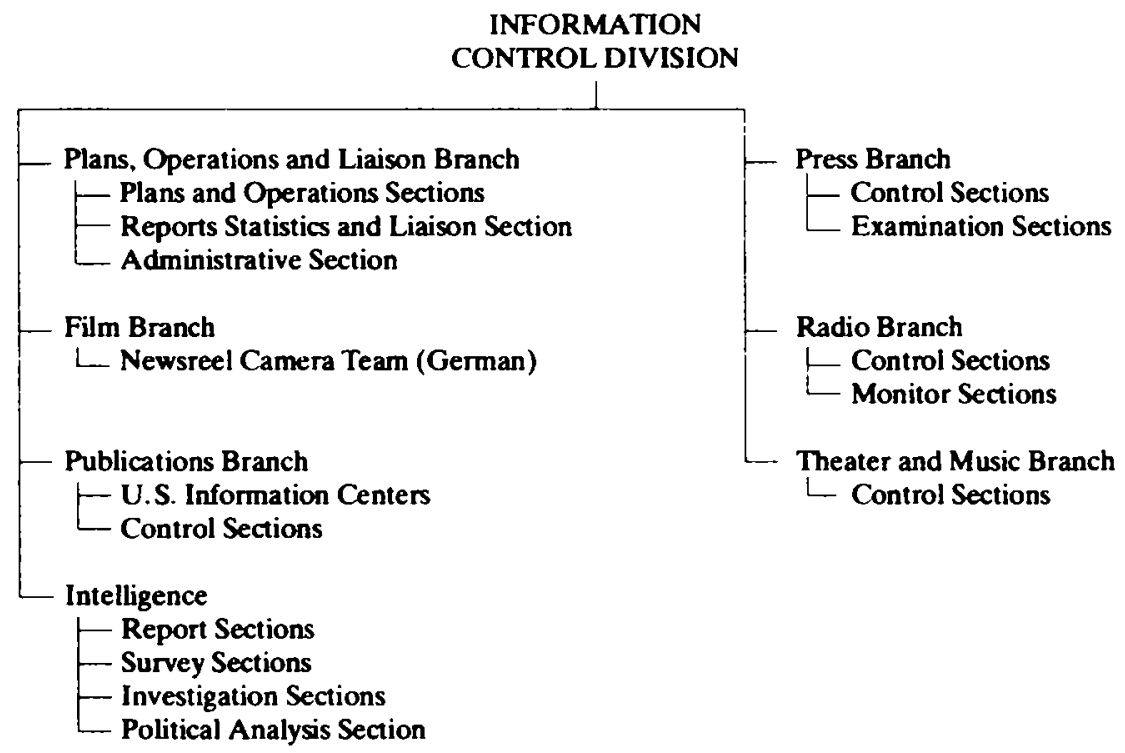

Im Gegensatz zu den meisten anderen Divisions verfügte ICD uber zahlreiche Außenstellen, wie die Übersicht 35 vom Juni 1947 verdeutlicht. ${ }^{131}$

Im Sommer 1947 wurde die Stabsabteilung auf zwei Positionen (Plans \& Operations Officer und Administrative Officer) gestrafft. Anfang 1948 wurden die Film Branch und die Theater and Music Branch, die schon zu Beginn der ICD-Tätigkeit eine gemeinsame Unterabteilung gebildet hatten, erneut in einer Branch vereinigt. Im zweiten Vierteljahr 1948 entfiel der Plans and Liaison Officer, und die Research Branch wurde in Media Investigation Unit umbenannt. General Order Nr. 8 vom 6. August $1948^{132}$ verlegte die Zuständigkeit für Theater und Musik in die Education and Cultural Relations Division; bei ICD verblieb die jetzt wieder eigenständige Film Branch. Bei der Organisationsstruktur von Ende 1948 (Übersicht 36) fällt auf, daß bis auf die Information Centers alle anderen AuBenstellen unter Aufsicht des Deputy Chief zusammengefaBt sind und daB sich ihre Zahl somit stark verringert hat. Sieben Information Control Officers nahmen jetzt die Aufgaben der Außenstellen aller Branches wahr. ${ }^{133}$

Die Press Branch hatte zunächst ubber Außenposten in Wiesbaden, Frankfurt, GieBen, Kassel und Darmstadt verfügt. Anfang 1948 kamen zusätzliche Außenstellen in Fulda und Marburg hinzu. Die Außenstellen waren meist mit einem Press Control Officer besetzt, dem - auBer in Darmstadt und Kassel - ein Press Assistant zur Seite stand. Ende 1948 entfielen alle Außenstellen bis auf Frankfurt wieder.

Die Radio Branch konzentrierte ihre Arbeit zunächst auf Radio Frankfurt. Nach Eröffnung der Studios in Wiesbaden und Kassel im Jahr 1948 unterhielt die Branch auch dort Außenposten. Sie hatte weiter Sections für den Frankfurter Kurzwellensender

131 OMGHE: Historical Report April - July 1947.

$1325 / 41-3 / 10$.

${ }^{133} 8 / 215-2 / 11$. 
Ubersicht 35: Organisation der Information Control Division, OMGHE, 30. Juni 1947

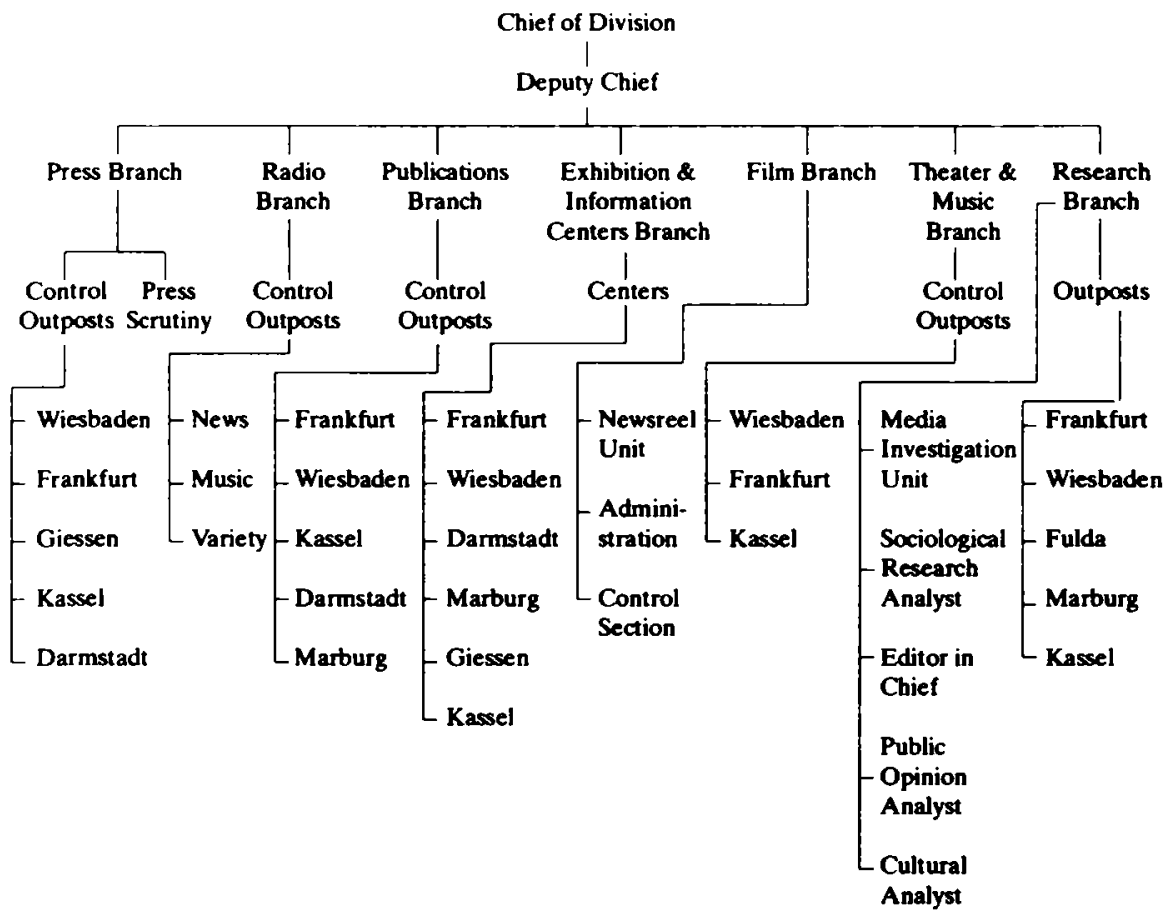

und Kabelradioprogramme (Wired Radio Program Distribution System) eingerichtet. Nach der Verabschiedung des Rundfunkgesetzes entfielen alle der Branch direkt zugeordneten AuBenposten.

Die Publications Branch unterhielt bis Ende 1948 Außenstellen in Frankfurt, Wiesbaden, Kassel, Darmstadt und Marburg, später dann nur noch in Frankfurt. Die Exhibitions and US Information Centers Branch unterhielt wăhrend der gesamten Zeit ihrer Tätigkeit in jedem der Center eine AuBenstelle. Die Film Branch in Frankfurt hatte seit April 1946 eine News Reel Unit, die eine deutsche Wochenschau produzierte. Nachdem die Branch ausgebaut war, verfügte sie uber eine für den Filmverleih zuständige Section und uber eine Control Section. Diese beiden Sections entfielen aber Ende 1948 wieder. Die Theater and Music Branch hatte AuBenstellen in Wiesbaden, Frankfurt und Kassel. Über die meisten Outposts verfügte zunächst die Intelligence Branch. Ihre acht Vertretungen befanden sich in Frankfurt, Darmstadt, Wiesbaden, Marburg, Gießen, Fulda, Bad Nauheim und Kassel. Als Research Branch hatte diese Organisationseinheit deren nur noch fünf: in Frankfurt, Wiesbaden, Fulda, Marburg und Kassel. Anfang 1948 kam kurzzeitig wieder eine Position in Darmstadt hinzu, die wie die anderen AuBenstellen im zweiten Vierteljahr 1948 wieder entfiel.

Die Division schrumpfte in der ersten Jahreshälfte 1949 weiter. Im Juni 1949 wurde sie ganz aufgelöst. ${ }^{134}$

$1348 / 42-3 / 11$ 
Ubersicht 36: Organisation der Information Services Division, OMGHE, 31. Dezember 1948

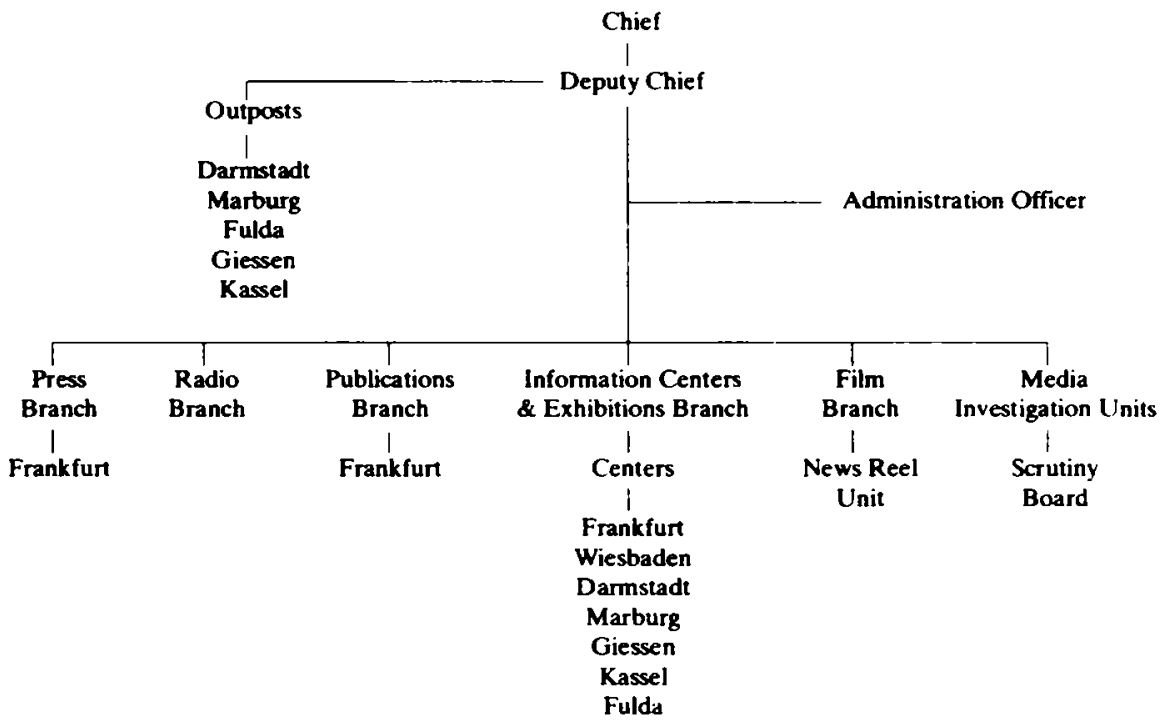

c) Personal

ICD, die einen Teil ihres Personals von DISCC und TISCC übernommen hatte, war fast während der gesamten Zeit ihrer Tätigkeit die größte Division in OMGHE, was die Zahl ihrer amerikanischen Mitarbeiter betraf. Mit 52 amerikanischen Mitarbeitern am 17. August 1946, also ein gutes halbes Jahr nach Beginn ihrer Tätigkeit, erreichte die Division ihren personellen Höchststand. Diese Zahl sank bis Herbst 1947 auf 36 ab. In dieser Zeit war die Economics Division vorübergehend die größte Division. Diese war jedoch um die Jahreswende $1947 / 48$ von Kürzungen betroffen, so daß zum März 1948 ICD, die ihre 36 amerikanischen Mitarbeiter behalten hatte, wieder stärkste Division war. Die Veränderungen im April 1948 ließen die Organisationsstruktur von ICD unangetastet, brachten aber eine Verringerung des amerikanischen Personals auf 26. Diese Zahl stieg während des Reorientation Program zunächst leicht an (1. Juli 1948: 28), sank aber auf 23 zum Jahreswechsel 1948/49. Am 2. Mai 1949 gehörten der Division dann nur noch acht Amerikaner an: der Chief, ein Mitarbeiter für Verwaltungsaufgaben und sechs Branch Chiefs.

Die größte Branch war die Intelligence/Research Branch. Sie umfaßte 194620 und 194718 US-Positionen. Die anderen sechs Branches umfaßten vier bis sieben Positionen. Abgesehen von der Exhibitions and US Information Centers Branch, der die amerikanischen Leiter der Amerika-Häuser angehörten, wurde der Personalstand der Branches bis Ende 1948 ungefähr halbiert, um dann 1949 auf je eine Position zusammenzuschrumpfen. Von dieser Reduzierung des Personals war auch die Exhibitions and US Information Centers Branch betroffen.

Von ihrer Aufstellung bis zum Februar 1947 wurde ICD von Anthony F. Kleitz geleitet. Ihn löste Bernard B. McMahon ab, der bis zum Juli 1947 amtierte. Zu diesem Zeitpunkt trat Dietrich Schroeder, der bisher Deputy Chief gewesen war, an die erste Stelle der Division. Vom Dezember 1947 bis November 1948 leitete dann Frederick N. Leonhard die Division. Leonhard war früher bei der Berliner Militärregierung tätig 
gewesen. Sein am 11. März 1948 zum Deputy Chief ernannter Stellvertreter Arthur Reef übernahm am 24. November 1948 die Amtsgeschäfte, die er bis zur Auflösung der Division führte. Zu seinem Stellvertreter wurde am 30. November 1948 Raymond J. Stover ernannt, der zuvor die Press Branch geleitet hatte.

In der Press Branch ersetzte ihn Vincent O. Anderson, der schon seit 1946 stellvertretender Leiter der Branch gewesen war. Wie Anderson gehörte auch der aus Palästina in die USA emigrierte Emil P. Jallouk der Division von ihrem Anfang bis zum Ende an. Er leitete zunächst die Plans, Operations and Liaison Branch, um nach deren Auflösung Chief der Exhibitions and US Information Centers Branch zu werden. Die Theater, Music and Film Branch leitete William P. Dubensky. Nachdem Theater and Music in die Education and Cultural Relations Division uberfuhrt waren, leitete Irvin C. Scarbeck die verbliebene Film Branch. Dieser war zunächst Jallouks Stellvertreter in der Plans, Operations and Liaison Branch gewesen.

Die Chefposition in der Publications Branch war zunächst unbesetzt. Sie wurde 1947 von Otto Albrecht eingenommen, der dann von dem österreichischen Emigranten Frederick A. Praeger, dem bisherigen Chief der Intelligence/Research Branch abgelöst wurde. Als Praeger am 21.September 1948 in die Vereinigten Staaten zurückkehrte, rückte sein bisheriger Stellvertreter Raymond Pagan zum Chief auf, der dieses Amt bis zum Ende der Division versah.

Die Radio Branch leitete zunächst Herbert C. Gross, bis er 1948 von Robert H. Lochner abgelöst wurde.

Die Leitung der großen Intelligence/Research Branch wechselte sehr oft. Auf D. L. McCarthy folgte Robert Browning, auf ihn der bisherige Deputy Chief F. A. Praeger. Als Praeger zur Publications Branch ging, kam Max H. Kimental, der die später in Media Investigation Unit umbenannte Einheit bis zum SchluB leitete.

\section{Intelligence Division}

\section{a) Aufgaben}

Ausgehend von einer OMGUS-Anweisung vom 22. Februar 1946 richtete OMGHE mit Letter Order Nr.82 vom 25. Mărz 1946 das Office of the Chief of Intelligence, OMGHE, ein. ${ }^{136}$ Obwohl Intelligence als Branch/Division tätig war, wurde dieser Name gewählt, um die Division von den Intelligence Branches und Intelligence Sections der anderen Divisions zu unterscheiden. Der Chief of Intelligence gehörte auch dem Stab des OMGHE-Direktors an. ${ }^{137}$

Die Aufgaben von Intelligence bestanden darin, „das Sammeln und Übermitteln von Nachrichtenmaterial zu koordinieren und für den OMGHE-Direktor Zusammenfassungen von Berichten und Analysen über Ereignisse, Entwicklungen und Trends zu erstellen. Um diese Aufgaben zu erfüllen, war Intelligence berechtigt, direkt von allen OMGHE-Einheiten nachrichtendienstliches Material anzufordern". ${ }^{138}$

Im Juni 1946 sandten bereits sechzig Stellen Informationen an die Division. Zusammenfassungen dieser Berichte schickte die hessische Intelligence an den Director of Intelligence von OMGUS (Berlin). Im Vordergrund stand allerdings die Information

\footnotetext{
$1368 / 53-2 / 2$ und $8 / 216-3 / 8$.

137 OMGHE: Historical Report October 1945 - June 1946.

$1388 / 53-2 / 2$
} 
des Direktors von OMGHE, vor allem was „Bedrohungen der Sicherheit der Militärregierung“ betraf. ${ }^{139}$

Die Arbeitsschritte von Intelligence bestanden aus:

a) Konzeption,

b) Nachrichtenbeschaffung,

c) Zusammenstellung,

d) Auswertung,

e) Einschätzung und

f) Informationsverteilung. ${ }^{140}$

Angesichts der zunächst dünnen Personaldecke handelte es sich dabei jedoch eher um eine Zielplanung denn um eine echte Arbeitsteilung.

Auf die Schwierigkeiten zu Beginn der Arbeit wies ein Memorandum des Chief of Intelligence vom 19. Oktober 1946 hin. ${ }^{141}$ Darin hieB es: „Die gegenwärtigen Leistungen der Intelligence Division sind mittelmäBig, unangemessen und unbefriedigend. Sie verdienen nicht den Titel ,Intelligence'. Dieser Zustand ist unerträglich und muB verändert werden.“ Als Ursachen für den Mißstand werden angeführt:

- Personalknappheit;

- der Aufbau der Division unter nicht qualifizierter Leitung;

- Kompetenzuberschneidungen mit anderen Divisions;

- die Überbetonung geheimdienstlicher Aktivitäten gegenüber seriöser Informationsbeschaffung, die zu einem „cloak and dagger stigm“ (dem Stigma vom Dolch im Gewande) der Division gefuhrt habe;

- Defizite bei den Informationszulieferern.

Hätten diese Probleme nicht bestanden, führte das Memorandum aus, so hätte beispielsweise der im Winter 1946/47 drohende wirtschaftliche Zusammenbruch fruher erkannt werden können. Abhilfe, so wurde weiter argumentiert, könnte vor allem die Aufstockung des Personalbestandes durch „handverlesene Mitarbeiter mit Spezialausbildung“" schaffen. Sollte diesem Vorschlag keine Folge geleistet werden, empfahl der Chief in seinem Memorandum die Auflösung der ganzen Division.

Das machte offensichtlich Eindruck: Ende 1946 wurden die im Memorandum entwikkelten Personalforderungen durch OMGUS und OMGHE erfullt. Daneben zogen sich andere Divisions von Intelligence Aufgaben zurück und kamen dadurch der Intelligence Division nicht mehr in die Quere. ${ }^{142}$ Seit Ende November 1946 erhielt Intelligence auch regelmäBige Situationsberichte der Liaison \& Security Offices ${ }^{143}$ - ein entsprechendes Frageraster, als Grundlage der Berichte, hatte die Division bereits am 23. Juli 1946 an die entsprechenden Stellen geschickt. ${ }^{144}$ Mit diesem Material konnte sie dann seit Anfang 1946 Tagesberichte ${ }^{145}$ (Daily Information Summaries, ISUM) für OMGUS erstellen. ${ }^{146}$ Neben den „overt informations“ der Liaison \& Security Offices

139 OMGHE: Historical Report October 1945 - June 1946.

140 Ebenda.

141 8/193-2/4.

142 So Economics Intelligence and Intelligence Branch von ICD; vgl. OMGUS-Hb, Teil Hessen B.5 und B.10.

143 OMGHE: Historical Report July - December 1946.

144 8/193-2/4.

145 Zum Teil in 8/193-2/4.

146 OMGHE: Historical Report July - December 1946. 
standen Intelligence auch die „covert informations“, die sie vom 970th $\mathrm{ClC}$ (Counter Intelligence Corps) erhielt, zur Verfügung. Der Eifer des Chief of Intelligence war OMGUS allerdings offenbar ein Stück zu weit gegangen: „Cooperate, do not compete!“ (,Zusammenarbeiten, nicht wetteifern!“) schrieben ihm die Berliner in einem Brief vom 20 . November 1946 ins Stammbuch. 147

Trotz des Übereifers gab es bei Intelligence offensichtlich auch Grund zu Klagen. Schon am 31. Januar 1947 monierte OMGUS eine „Verzögerung in der Berichtsübermittlung" von OMGHE. Der Chief of Intelligence entschuldigte dies - nach einem Seitenhieb auf die Liaison \& Security Offices und deren Berichterstattung - mit schwierigen Wetterverhältnissen: da die gesamte Nachrichtenübermittlung uber Kuriere liefe, hätten wetterbedingte Verkehrsstörungen die Verzögerung verursacht. ${ }^{148}$ Obwohl der erste Vierteljahresbericht 1947 von OMGHE positiv über den Organisationsaufbau von Intelligence berichtet, ${ }^{1 / 9}$ scheinen bestimmte Probleme weiter bestanden zu haben. In einem Rundschreiben an die Liaison \& Security Offices vom 22. Juli 1947 weist der Chief of Intelligence darauf hin, daß deutsche Mitarbeiter keine „Investigators“ sein könnten, sondern nur wie Zeitungsreporter eingesetzt werden dürften. ${ }^{150}$ Die Liaison \& Security Offices hatten offenbar auf Deutsche als Beschaffer von Informationen zurückgegriffen, da es weiterhin an geeignetem amerikanischen Personal fehlte, wie der dritte Vierteljahresbericht von 1947 beklagt. ${ }^{151}$ Die Liaison \& Security Officers seien „kein befriedigender Ersatz für ausgebildetes Nachrichtendienstpersonal", heiBt es schlieBlich im vierten Vierteljahresbericht von 1947. ${ }^{152}$ Auch die Beschaffung geheimdienstlicher Informationen machte Probleme. Intelligence war hier auf CIC angewiesen und deshalb von den Personalkürzungen, die CIC Ende 1947 hinnehmen muBte, in seiner Arbeit mitbetroffen. ${ }^{153}$ Diese Probleme verloren jedoch in den letzten zwei Jahren der OMGHE-Tatigkeit ihre Schärfe: die Politik der Militärregierung verlagerte sich zunehmend von Überwachung und Kontrolle hin zu Medienarbeit und „reorientation“.

\section{b) Organisation}

Der Aufbau der Intelligence Division zog sich durch das ganze Jahr 1946. Erst zum Jahresende war sie ,in der Lage, ihre Aufgaben voll wahrzunehmen“, heißt es im Bericht uber das zweite Halbjahr 1946. ${ }^{154}$ Nach der Bildung der Division fand am 20. Mai 1946 eine Konferenz über ihre künftige Struktur statt, ${ }^{155}$ die allerdings zunächst keine Folgen für den praktischen Aufbau hatte: noch am 1.Juli 1946 setzte sich die Division lediglich aus dem Chief und Enlisted Men (Mannschaftsdienstgrade) zusammen. ${ }^{156}$ Erst nach den Personalzuweisungen Ende 1946 konnte die Division eine tatsächliche Organisationsgliederung vornehmen. Diese Organisationsgliederung von Anfang 1947 unterscheidet sich allerdings noch deutlich von der Struktur der anderen Divisions: ${ }^{157}$

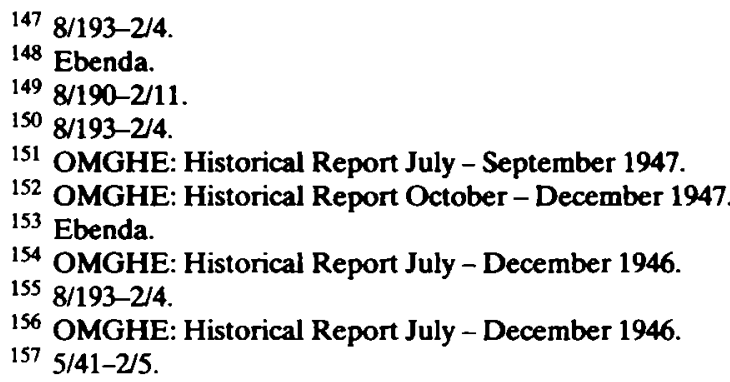


Ubersicht 37: Organisation der Intelligence Division, OMGHE, Anfang 1947

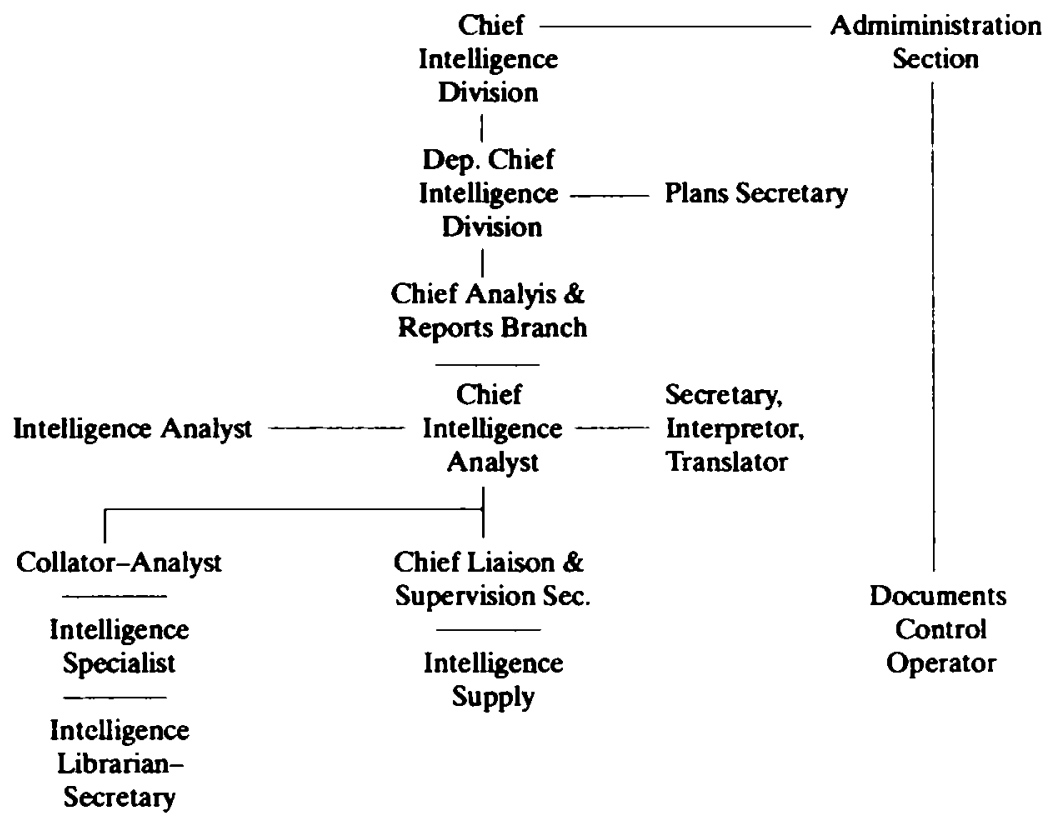

Anstelle einer Branch-Aufteilung sind alle einzelnen Positionen mit ihren Aufgaben benannt. Erst zum Ende der ersten Jahreshălfte 1947 zeigte sich die Division in der üblichen Struktur: ${ }^{1.58}$

Ubersicht 38: Organisation der Intelligence Division, OMGHE, 30. Juni 1947

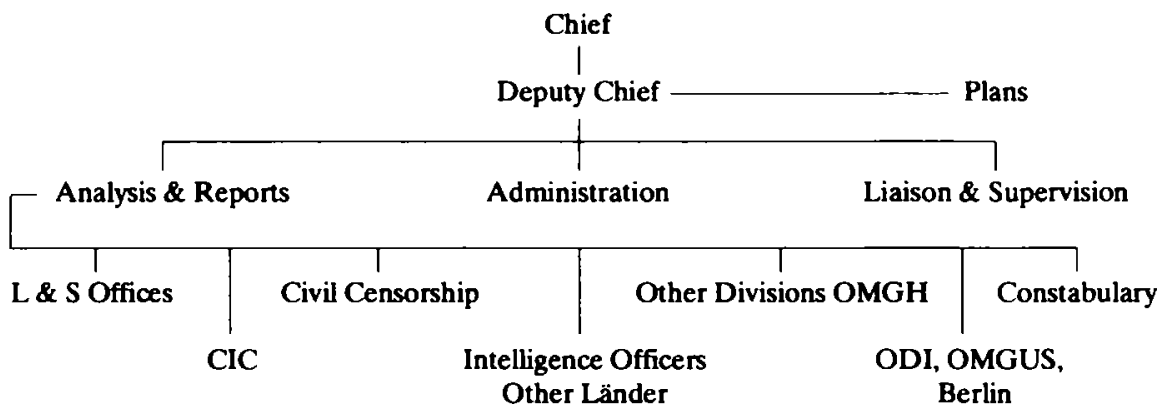

Die Division war in vier Branches gegliedert. Für die Planung blieb der Deputy Chief zuständig; der Verwaltungsbereich, der anfänglich direkt an den Chief angebunden war, wurde jetzt in die anderen Branches eingereiht. Die Analysis and Reports Branch war die Schaltstelle für das Nachrichtenwesen. Bei ihr gingen die Berichte ein. Sie wertete sie aus und gab die Ergebnisse weiter. Die Organisationsgliederung zeigt auch

158 OMGHE: Historical Report April - July; 5/41-2/5. 
die zahlreichen Stellen, die entweder an die Branch Berichte abgaben oder Berichtsergebnisse von ihr erhielten. In der Liaison \& Supervision Branch waren die Kontrollfunktionen gebündelt. Sie überwachte das Berichtswesen und nahm „Feldaufgaben“ wahr. Diese Organisationsstruktur blieb bis zum Frühjahr 1949 erhalten, als die Division auf zwei Stellen reduziert wurde.

\section{c) Personal}

Wie bereits erwähnt, betrug die Zahl der fest bei der Intelligence Division beschäftigten Amerikaner im Sommer 1946 nur drei. Erst im Frühjahr 1947 erreichte die Division ihre Sollstärke von 16, die zum Herbst wieder auf 14 verringert wurde. Diese Zahl blieb bis Ende 1948 fast konstant; bei den von OMGUS im März 1948 angeordneten allgemeinen Personalkürzungen kam die Intelligence Division ungeschoren davon. Zum Jahresende 1948 wurden ihre Stellen nur leicht - um zwei - auf zwölf gekürzt. Bis zum Ende der Besatzungszeit schrumpfte der amerikanische Personalbestand der Division auf zwei Positionen, den Division Chief und den Chief der Analysis and Reports Branch zusammen.

Bis 1947 waren 15 Deutsche für die Division tätig. In diesem Jahr entfielen alle ihre Stellen. Am 30. Oktober 1947 wurde ihre Zahl bereits mit Null angegeben. Diese Entwicklung hatte ihre Ursache wohl vor allen Dingen im MiBtrauen der Amerikaner gegenüber der Mitarbeit von Deutschen im Intelligence Bereich, wie es in dem erwähnten Brief des Division Chief vom 22. Juli 1947 zum Ausdruck kam. ${ }^{159}$ Sie widerspricht jedoch der Gesamttendenz der amerikanischen Besatzungspolitik, die zunehmend auf deutsche Mithilfe im Kalten Krieg baute. Eine mögliche Erklärung wäre, daß es sich bei den deutschen Mitarbeitern um linke Antifaschisten gehandelt hatte, die in der neuen weltpolitischen Konstellation verdächtig wurden.

Mit der Anordnung zur Bildung einer Intelligence Einheit wurde gleichzeitig James O'Steen zum Chief ernannt. Er übte dieses Amt bis zum Jahresende 1948 aus. Seit Januar 1949 leitete dann sein bisheriger Stellvertreter Robert A. Cunningham die Division. Die Stellvertreter-Stelle war bis zum Amtsantritt Cunninghams Anfang 1947 unbesetzt gewesen.

\section{Land Resource Board}

Circular Nr.71, USFET, vom 23. Mai $1946^{160}$ richtete in der amerikanischen Besatzungszone Resource Boards ein. Diese Boards sollten die Versorgung des amerikanischen Militärs mit dem Bedarf der Militärregierung und der wirtschaftlichen Situation in Deutschland abstimmen sowie in Streitfällen vermitteln. Sie waren paritätisch aus Vertretern der Armee und der Militärregierung zusammengesetzt. Neben dem zonalen Board agierten Boards auf Landesebene und auf lokaler Ebene. Bei den Local Boards erfolgte die räumliche Einteilung in Anlehnung an die Bezirke der Military Posts. Die Boards nahmen zum 15. Juni 1946 ihre Arbeit auf.

Dem hessischen Land Resource Board gehörten OMGHE-Direktor J. R. Newman und Col. Paul E. Ruestow von den USAFE Headquarters (1947: Brigadier General Fay Prickett, 1 st Constabulary Brigade) an. Nicht stimmberechtigter Sekretär war Major Milton Waltcher, ein Mitarbeiter der Economics Division von OMGHE (1947:

$1598 / 193-2 / 4$.

$1605 / 41-2 / 7 ; 8 / 214-1 / 16$. 
William P. Weber, Economics Division, OMGHE). Der Land Resource Board hatte folgende Arbeitsausschüsse (Permanent Committees):

- Supply and Materials (Versorgung und Materialien), Mitglieder: Olav Maseng, Deputy Chief of Economics Division, OMGHE; Col. Woodward B. Mann, USAFE Headquarters.

- Real Estate Property (zuständig für Liegenschaften), Mitglieder: Samuel S. Graham, Chief of Industry Branch, Economics Division, OMGHE; Lt.Col. M. Falkow, USAFE Headquarters (1947: Col. Paul S. Blair, USAFE).

- Manpower Allocation (Arbeitskräfte), Mitglieder: Glenn E. Garrett, Chief of Manpower Division, OMGHE; Col. John W. White, USAFE Headquarters (1947: Lt.Col. Frank A. Hartmann, USAFE).

Local Resource Boards arbeiteten in Darmstadt, Wiesbaden, Kassel und Frankfurt. 1947 kamen weitere Local Boards in Fulda und GieBen hinzu. ${ }^{161}$

Die Resource Boards griffen bei Streitfällen zwischen Armee und Militärregierung ein. Die Historical Reports von OMGHE berichten über zwei landesweit bedeutende Auseinandersetzungen:

- In Griesheim (bei Frankfurt) hatte eine Ordonance Unit der Armee ein chemisches Werk der IG-Farben requiriert, das Kohlen-Elektroden und Farben hergestellt hatte. OMGHE hielt die Wiederaufnahme der Produktion dieser Güter für sehr wichtig und drängte die Armeeinheit zum Verlassen des Werkes. Der Konflikt zog sich fast ein Jahr hin. Erst im Juni 1947 fand der Land Resource Board einen KompromiB: die Einheit sollte in Neubauten untergebracht werden. ${ }^{162}$

- In Bensheim hatte eine Engineer Unit der Armee eine Fabrik in Beschlag genommen, die Küchengegenstände und Papier produziert hatte. Der Darmstädter Local Resource Board beschloß, daß die Armeeinheit die Fabrik zu verlassen hätte, damit diese die Produktion wieder aufnehmen könne. ${ }^{163}$

\section{Legal Division}

a) Aufgaben

Die Legal Division hatte drei Hauptaufgaben:

a) die Gerichtsbarkeit der Militärregierung;

b) die Kontrolle des hessischen Justizsystems einschlieBlich der Gefängnisse;

c) die Kontrolle der hessischen Gesetzgebung (zusammen mit der Civil Administration Division).

Zu a) Die Gerichtsbarkeit der Militärregierung

Nach dem Einmarsch der Amerikaner waren zunächst alle Militärregierungsgerichte (Field Military Government Courts) von den Truppenkommandanten ernannt worden. Das Hauptquartier der 7. Armee hatte die höheren Instanzen der Gerichte im hessischen Bereich ernannt. Am 1. Januar 1946 gingen die gesamten Emennungsrechte (auch für die Revisionsinstanzen) an den Direktor von OMGHE über.

161 Zusammensetzung in 8/214-1/16 und OMGHE: Historical Report April - July 1947, Annex.

162 OMGHE: Historical Report July - December 1946; OMGHE: Historical Report April - July 1947

163 OMGHE: Historical Report July - December 1946. 
Die MG Courts waren laut MG Ordinance Nr. 2 zuständig für alle Angehörigen von Staaten der Vereinten Nationen, zivile US-Amerikaner und für Deutsche, die gegen Anordnungen der Militärregierung oder der alliierten Streitkräfte verstoßen hatten. Es bestanden drei verschiedene Ebenen von MG Courts:

- Summary Courts mit einem Offizier oder US-Zivilisten, die Gefängnisstrafen bis zu einem Jahr und Geldstrafen bis zur Höhe von 10000 RM aussprechen konnten;

- Intermediate Courts mit einem bis drei Offizieren, die Gefängnisstrafen bis zu zehn Jahren und Geldstrafen bis 100000 RM verhängen konnten;

- General Courts mit mindestens drei Offizieren, die alle Strafen, auch die Todesstrafe, verhängen konnten. ${ }^{164}$

Richter bei den Courts waren zum geringeren Teil Angehörige der Legal Division, zum größeren Teil aber andere Angehörige der Militärregierung, die das Richteramt „nebenberuflich" versahen. „Full-time Courts" bestanden in Kassel, Gießen, Wiesbaden, Frankfurt und Darmstadt. Nach der Verbesserung der Transport- und Kommunikationsmöglichkeiten versuchte die Division möglichst viele Gerichtsverfahren auf diese Standorte zu konzentrieren. ${ }^{165}$

Ubersicht 39: Military Government Courts in Hessen, Oktober 1945 - Mai $1946^{166}$

$\begin{array}{lrrrrrrrr} & \text { Okt } & \text { Nov } & \text { Dez } & \text { Jan } & \text { Feb } & \text { Mărz } & \text { April } & \text { Mai } \\ \text { General } & 0 & 0 & 0 & 1 & 1 & 1 & 3 & 3 \\ \text { Intermediate } & 4 & 4 & 6 & 6 & 6 & 6 & 6 & 6 \\ \text { Summary } & 38 & 35 & 39 & 39 & 39 & 39 & 39 & 39\end{array}$

Die Legal Division uberprüfte alle Urteile der Militärregierungsgerichte und nahm dabei in Einzelfällen ihr Recht wahr, Strafe und Urteil zu modifizieren. Am 11.Juli 1946 bildete sie auch einen BegnadigungsausschuB, dem ein leitender Mitarbeiter der Division, ein Gefängnisoffizier und der deutsche Leiter des hessischen Gefăngniswesens angehörten.

Probleme traten dadurch auf, daB unter dem Militärregierungspersonal, aus dessen Reihen die Richter berufen wurden, zu wenig ausgebildete Juristen waren. So sollte beispielsweise in den Intermediate Courts mindestens ein Jurist sitzen. Da diese Minimalforderung oft nicht erfült werden konnte, muBte die Division ein Ausbildungsprogramm durchfüren, was wiederum ihre personellen Möglichkeiten uberstieg. ${ }^{167}$ Zur besseren Kontrolle der MG Courts teilte Legal Division das Land Hessen in der zweiten Jahreshälfte 1946 in vier Bezirke (Field Legal Areas) mit je einem Senior Legal Officer ein. ${ }^{168}$

Vor besondere Probleme stellten die MG Courts Verbrechen von Displaced Persons. Zum einen waren die DPs aufgrund ihrer besonderen Situation häufig in Straftaten verwickelt, und zum anderen bestand Unklarheit dartber, was mit verurteilten DPs geschehen sollte. Sie wurden zunächst einem gesonderten Strafvollzug unterstellt.

Nachdem am 15. Februar 1946 der Security Review Board der 7. Armee aufgelöst worden war, bildete OMGHE mit Letter Order vom 29. März 1946 einen eigenen Security

\footnotetext{
164 OMGHE: Historical Report October 1945 - June 1946.

${ }^{165}$ Ebenda.

166 OMGHE: Historical Report October 1945 - June 1946, Annex.

$16717 / 211-3 / 3$.

168 OMGHE: Historical Report July - December 1946.
} 
Review Board, der die Aufgabe hatte, auf Antrag die RechtmäBigkeit von Einweisungen in Internierungslager (Automatic Arrest) zu überprüfen. Dem Board gehörten drei in Wiesbaden stationierte Mitglieder (der Vorsitzende, ein CIC-Mitarbeiter und ein Offizier der Legal Division) und fünf Mitarbeiter des Lagers Darmstadt an. Zu seiner Unterstützung wurden in allen hessischen Kreisen deutsche Security Review Boards eingerichtet, die für den amerikanischen Board Material bereit stellen sollten. Um eine Verringerung der Lagerinsassen zu erreichen, drängte OMGUS auf eine schnelle Bearbeitung: der Security Review Board sollte wöchentlich 800 - 1000 Fälle bearbeiten. In der Folgezeit wurden weniger als $10 \%$ der Entlassungsanträge zurückgewiesen. ${ }^{169}$ Bis zum 30. Juni 1946 hatte der Board bereits 7480 Fälle behandelt und 6942 Freilassungen verfügt. ${ }^{170}$

Schon 1947 zeichnete sich ab, daß die MG Courts Kompetenzen an deutsche Gerichte abgeben würden. Zum einen sollte dadurch die deutsche Verantwortlichkeit gestärkt werden, zum anderen überstieg beispielsweise die Zahl der Diebstahlsdelikte aus USDepots ohnehin die Leistungsfähigkeit der MG Courts. So wurden im Sommer 1947 Fälle mit einer Schadenshöhe bis zu 50 Dollar in deutsche Zuständigkeit übergeben. ${ }^{171}$ Im Januar 1948 wurden durch MG Ordinance Nr. 23 die Habeas-Corpus-Bestimmungen für die Militärgerichtsbarkeit eingeführt. Zu einer weiteren Entlastung der MG Courts trug bei, daB seit April 1948 deutsche Gerichte in beschränktem Umfang Vergehen von Angehörigen ausländischer Staaten - mit Ausnahme von Armeeangehörigen - behandeln durften. Zu dieser Zeit wurden auch die Bestimmungen über Fragebogenfälschungen, die in die Zuständigkeit der MG Courts fielen, entschärft.

Am 26. Oktober 1948 wurde die Militärregierungsgerichtsbarkeit in der amerikanischen Zone völlig neu organisiert. Sie ging jetzt aus den Händen der Landesmilitärregierung in die Zuständigkeit einer unabhängigen OMGUS-Stelle über. Im neuen OMGUSSystem bildete Hessen die Distrikte III und IV. Die MG Courts von OMGHE hatten sich seit diesem Zeitpunkt nur noch mit weiter zurückliegenden Verfahren und deren Kontrolle zu befassen und konnten dadurch ihre Tătigkeit weitgehend beenden.

$\mathrm{Zu} \mathrm{b)} \mathrm{Die} \mathrm{Kontrolle} \mathrm{der} \mathrm{deutschen} \mathrm{Justiz}$

Hatten zunächst die Offiziere der Detachments die Richter und das Personal im Justizwesen bestimmt, so kontrollierte später die Legal Division den Aufbau eines demokratischen und inhaltlich wie personell entnazifizierten Rechtswesens in Hessen über das Justizministerium. Dieses Ministerium wurde aufgrund Organizational Direction Nr. 2 vom 14. Oktober 1945 (mit einer Ergänzung durch Organizational Direction Nr. 13 vom 7. Januar 1946) gebildet. Das Justizministerium übernahm alle Befugnisse im Rechtswesen, die zuvor bei den Provinzregierungen gelegen hatten. Mit Organizational Direction Nr. 10 vom 15. Dezember 1945 entzog OMGHE den Kreisund Regierungsbezirksdetachments gleichfalls alle Befugnisse in diesem Bereich.

Die bereits eingesetzten Gerichte setzten unter neuer Aufsicht ihre Arbeit fort. Als erste Gerichte in Hessen hatten am 4. Juni 1945 die Amtsgerichte in Limburg und Camberg ihre Arbeit wieder aufgenommen. Der Aufbau des hessischen Gerichtswesens war zum 1. April 1946 abgeschlossen. Am 8. März des gleichen Jahres wurde das Oberlandesgericht Frankfurt mit Nebenstellen in Darmstadt und Gießen eröffnet; in der zweiten Märzhälfte nahmen die letzten Amtsgerichte ihre Arbeit auf.

169 OMGHE: Historical Report October 1945 - June 1946.

170 OMGHE: Historical Report July - December 1946.

171 OMGHE: Historical Report April - July 1947. 
Die Legal Division arbeitete gemeinsam mit dem Justizministerium ein spezielles Entnazifizierungs- und Zulassungsverfahren für die juristischen Berufe aus, das ohne die Einschaltung örtlicher Militärregierungsstellen auskam. ${ }^{172}$ Im Jahre 1948 erhielten die deutschen Gerichte dann das Recht, auch Kriegsverbrechen zu behandeln.

Die Zuständigkeit für die deutschen Gefängnisse ging im Februar 1948 von der Public Safety Division auf die Legal Division über. Sie kontrollierte über das Justizministerum die damals 43 hessischen Gefängnisse. Dabei nahm sie AnstoB an einigen Eigenheiten des deutschen Gefängniswesens (Einzelzellen, Beschränkung der Freizeitaktivität auf Hofgang, mangelhafte Trennung von Geschlechtern und Generationen) und verlangte die Vorlage neuer Konzepte. ${ }^{173}$ Dem akuten Problem der anhaltenden Überbelegung der Gefängnisse versuchte man durch die Förderung von Neubauten und durch Amnestien (z. B. Christmas Amnesty 1946) zu begegnen. ${ }^{174}$

$\mathrm{Zu} \mathrm{c)} \mathrm{Die} \mathrm{Kontrolle} \mathrm{der} \mathrm{hessischen} \mathrm{Gesetzgebung}$

„Im legislativen Bereich besteht die Aufgabe der Legal Division darin, den Director zu beraten und für ihn alle gesetzgeberischen und quasigesetzgeberischen Maßnahmen auf ihre rechtliche Zulässigkeit und auf ihre Übereinstimmung mit den Richtlinien, Gesetzen und Verordnungen des Kontrollrats, der Vereinigten Staaten und der Militärregierung zu überprüfen “, heißt es im ersten großen Historical Report. ${ }^{175}$ Im einzelnen setzte sich die Legal Division dafür ein, daB nazistische Inhalte aus den bestehenden Gesetzen getilgt wurden und daB das neugebildete Land Hessen ein einheitliches Rechtssystem erhielt. In der ersten Zeit mußten Gesetzentwiurfe vor der Verabschiedung der Legal Division vorgelegt werden, die ihrerseits wiederum dem Direktor von OMGHE eine Stellungnahme empfahl. Als sich nach der Landtagswahl vom 1. Dezember 1946 eine steigende Flut von Gesetzentwürfen abzeichnete, wurde diese Prozedur auf Seiten von OMGHE formalisiert.

Ausgehend von einem Memorandum des Assistant Executive Officer Robert Wallach vom 11. Dezember $1946^{176}$ legte OMGHE mit Organizational Direction Nr.24 vom 14. März 1946 folgendes Verfahren fest: ${ }^{177}$

- OMGHE erhält vor der Beschlußfassung des Landtages alle Gesetzentwürfe zu Händen der Legal Division.

- Legal Division schickt Kopien an die Civil Administration Division und an die Division, deren Tätigkeitsbereich von dem Gesetzentwurf beruhrt wird.

- Zum 14. März 1946 wird ein Legislative Committee gebildet, das aus den Chiefs der Legal und der Civil Administration Division besteht. Dieses Committee zieht zu seinen Beratungen die Chiefs der betroffenen Divisions hinzu. Die Civil Administration Division leitet die Stellungnahme des Committee dann an die Landesregierung weiter. Später wurde das Verfahren insoweit präzisiert, daB sich das Committee nur noch mit Gesetzentwürfen beschäftigte, die irgendwelche Widersprüche hervorgerufen hatten. ${ }^{178}$

- Kopien von im Landtag beschlossenen Gesetzen leitet die Legal Division an die Legal Division von OMGUS und an das Regional Government Coordinating Office weiter.

172 OMGHE: Historical Report October 1945 - June 1946 und ebenda, Annex.

173 OMGHE: Historical Report October 1945 - June 1946.

174 OMGHE: Historical Report January - March 1947, OMGHE: Historical Report July - September.

175 OMGHE: Historical Report October 1945 - June 1946.

176 17/211-3/3.

177 5/41-2/5.

178 OMGHE: Historical Report January - March 1947. 
Allerdings konnte OMGHE nicht zu allen Gesetzentwürfen Stellung beziehen: Bedeutsamere Vorhaben wurden von OMGUS behandelt. So blieb OMGHE beispielsweise zur Intervention von OMGUS gegen den Sozialisierungsartikel in der Hessischen Verfassung nur ubrig, in ihrem Historical Report zu kommentieren: „Das Resultat [der OMGUS-Intervention] war fast ein Chaos. "179 Am 30. Dezember 1947 forderte J.R. Newman von OMGUS emeut eine grundsätzliche Stellungnahme in der sich hinziehenden Auseinandersetzung. ${ }^{180}$

Die Einwände von OMGHE gegen Gesetzentwürfe hatten zwei Schwerpunkte:

- zum einen verlangte die Militärregierung bei allen Gesetzen, die Sozialisierungsmaßnahmen zum Ziel hatten oder den Beschäftigten Mitbestimmungsrechte einräumten, die Zurückstellung und das Abwarten von Entscheidungen einer deuschen Zentralinstanz;

- zum anderen wies sie zahlreiche Gesetze zurück, die mit berufsständischen Regelungen (Zulassungsverfahren, Zwangskörperschaften o. a.) die Gewerbefreiheit einschränkten. Da praktisch alle in diesem Bereich vorgelegten Gesetze zurlickgewiesen wurden, erlieB OMGHE noch am 19. November 1948 eine förmliche Direktive für Berufsorganisationen, gegen die kein Gesetzentwurf verstoßen durfte. ${ }^{181}$

Neben diesen drei Hauptaufgaben beriet die Legal Division die anderen Divisions von OMGHE und auch deutsche Stellen in Rechts- und Verfassungsfragen.

\section{b) Organisation}

Nach ihrer Bildung umfaßte die Legal Division zunächst sechs Branches, die zuständig waren für.

- Berichte und Protokollwesen,

- deutsche Gefängnisse,

- Militärregierungsgerichte,

- deutsche Justiz,

- Gesetzgebung und Beratung und

- Verfahren von deutschen Internierten.

Ubersicht 40: Organisation der Legal Division, OMGHE, Juni $1946^{182}$

LEGAL DIVISION

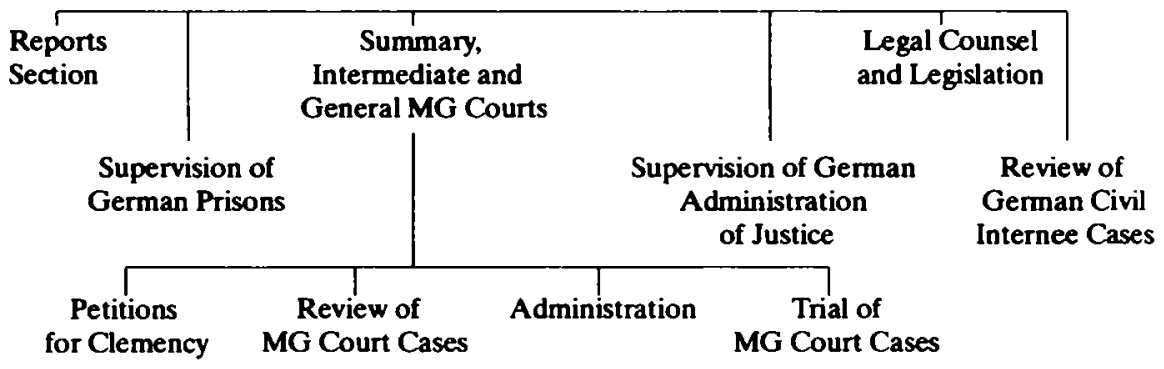

179 OMGHE: Historical Report July - December 1946.

180 17/211-3/3.

181 OMGHE: Historical Report 1948; vgl. die Auseinandersetzungen um das Gewerbezulassungsgesetz in Akten zur Vorgeschichte 4, S. $1011 \mathrm{ff}$. und $1025 \mathrm{ff}$.

182 OMGHE: Historical Report October 1945 - June 1946. 
Die MG Courts Branch war zudem in vier Sections (Überprüung von MG CourtsVerfahren, Durchführung von MG Courts-Verfahren, Gnadengesuche und Verwaltung) unterteilt (Übersicht 40).

In der zweiten Jahreshälfte 1946 teilte die Division dann die MG Courts in fünf Bezirke auf und bildete entsprechende Field Teams mit zunächst je drei Mitarbeitern (ab 1. Juli 1947 in Frankfurt, Wiesbaden und Darmstadt nur noch zwei Mitarbeiter). Darüber hinaus unterhielt sie eine Review Section, die die Revisionsfälle überprüfte und die Wiederaufnahme von Verfahren vorbereitete. Getrennt davon arbeitete die Abteilung des Anklagevertreters. Die German Administration of Justice Branch richtete zum 1. Mai 1947 eine AuBenstelle in Marburg ein, um die deutschen Gerichte in Nordund Mittelhessen besser überwachen zu können. Die einzelnen Branches hatten jetzt ihr Berichtswesen selbst zu verwalten, eine eigenständige Reports Section entfiel. Für Fälle von deutschen Internierten war jetzt ausschlieBlich der am 29. März 1946 gebildete Security Review Board zuständig, die entsprechende Branch der Legal Division entfiel. Begnadigungs- und Bewährungsfragen wurden jetzt nicht mehr im Rahmen der MG Courts behandelt. Eine entsprechende Section war dem Stabsbereich der Division angegliedert.

Die Division hatte so bis Anfang 1948 folgende Struktur. ${ }^{183}$

Ubersicht 41: Organisation der Legal Division, OMGHE, 30. Juni 1947

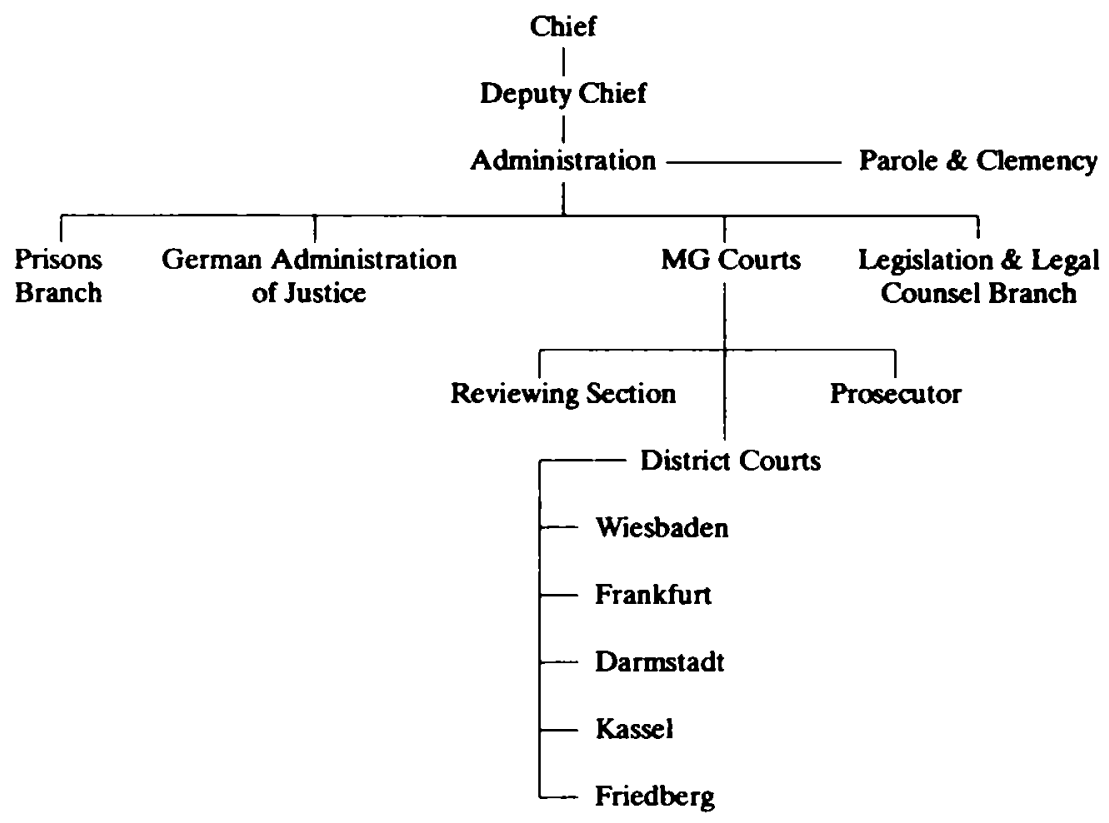

Aufgrund der abnehmenden Belastung konnten im ersten Vierteljahr 1948 die German Administration of Justice und die Legislation and Legal Advice Branch zusammengelegt werden. Die neugeschaffene Branch hatte Sections für Gesetzgebung, Beratung und deutsche Strafjustiz. Zur gleichen Zeit wurden die Überprüfung der MG

183 OMGHE: Historical Report April - July 1947. 
Courts und die Anklage-Abteilung aus der MG Courts Branch herausgenommen und der Stabsabteilung angegliedert.

Damit hatte die Division für kurze Zeit folgende Struktur. ${ }^{184}$

Ubersicht 42: Organisation der Legal Division, OMGHE, 31. Mai 1948

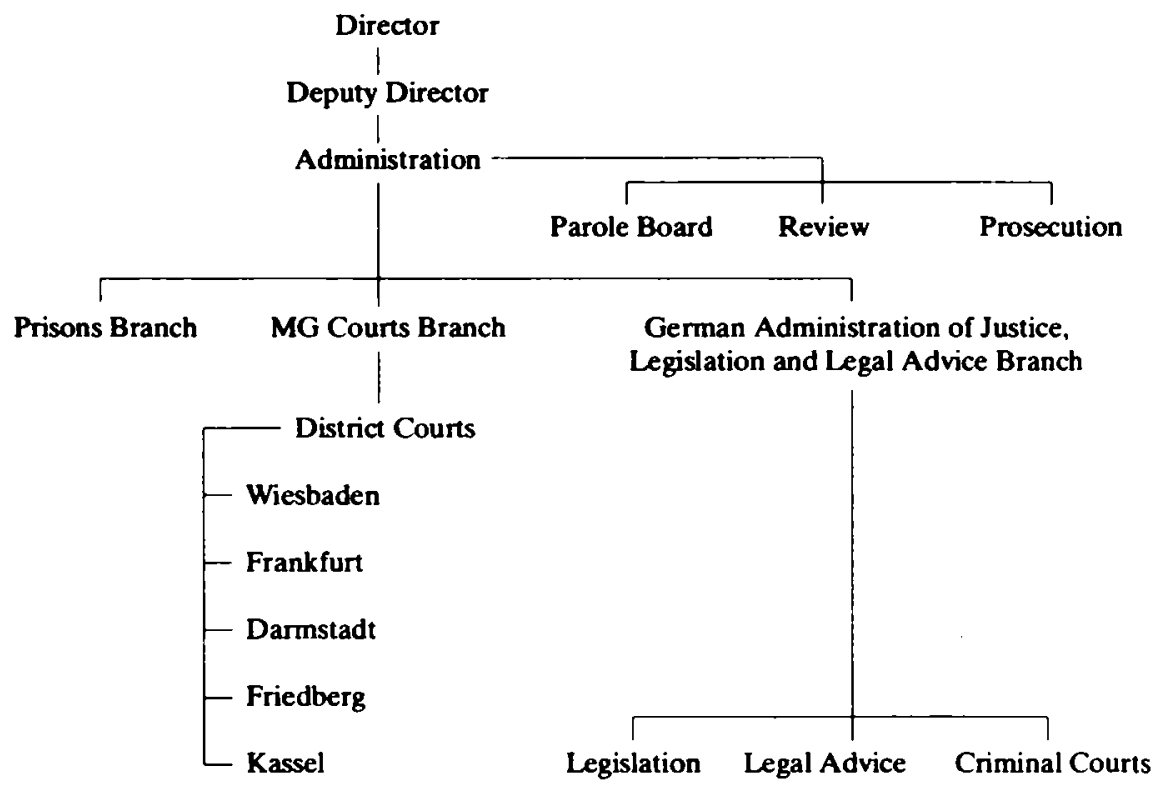

Schon am 30. Juni 1948 wurde eine weitere Straffung vorgenommen. Im Bereich der Militärregierungsgerichtsbarkeit war die Legal Division jetzt nur noch für Begnadigungs- und Bewährungsfragen zuständig. Diese Bereiche wurden mit der Prisons Branch zu einer Branch zusammengelegt. Die Organisationsgliederung nimmt den offiziellen Wegfall der OMGHE zugehörigen MG Courts Branch am 26. Oktober 1948 schon zum 30.Juni 1948 vorweg. Weiter war zu diesem Zeitpunkt die eigenständige Verwaltungsabteilung entfallen. Die Division hatte vom Sommer 1948 bis zur Auflösung von OMGHE folgende Struktur (wobei Anfang 1949 auch noch die Position des Deputy Chief entfiel): ${ }^{185}$

Ubersicht 43: Organisation der Legal Division, OMGHE, 30. Juni 1948

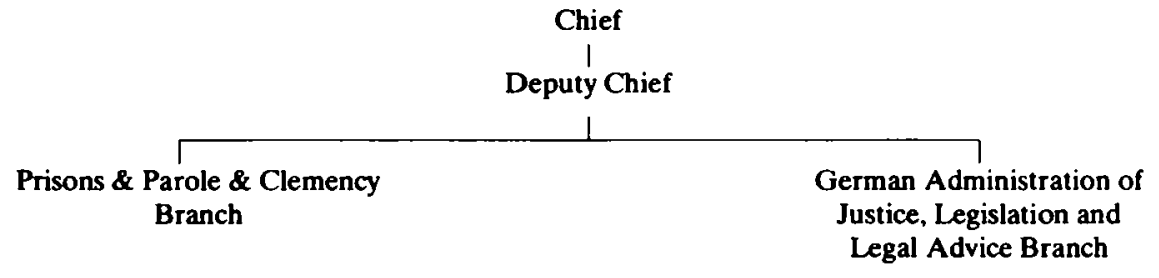

$1845 / 41-2 / 17$.

185 5/41-3/10. 


\section{c) Personal}

Die Legal Division gehörte zu den gröBten Divisions von OMGHE. Mit der Zahl der amerikanischen Mitarbeiter nahm sie zumeist die dritte Position ein (hinter ICD und Economics Division). Im Sommer 1946 zählte sie 37 amerikanische Mitarbeiter. Diese Zahl blieb ein Jahr lang konstant, um zum 30. Oktober 1947 auf 30 abzusinken. Zum 1. Januar 1948 waren 26, zum 30. Juni 25 und zum 31. Dezember 1948 dann nur noch zehn Amerikaner bei der Legal Division beschäftigt. Diese Zahl verringerte sich 1949 dann auf drei. Zunächst arbeiteten 20 deutsche Mitarbeiter bei der Division. Deren Zahl erhöhte sich 1947 vorübergehend bis auf 30, um dann 1948 wieder auf 17 abzusinken.

Von Juli 1945 bis Dezember 1946 leitete Krit G. Logsdon die Legal Division. Sein Nachfolger bis Sommer 1949 war Franklin J. Potter. Potter war schon seit der Aufstellung von E1 A2 in Frankreich als Legal Officer dabei und war Logsdons Stellvertreter gewesen. Sein Stellvertreter wiederum war Marc J. Robinson. Von Juli bis September 1949 war der langjährige Executive Officer Emest K. Neumann Chief der Division. Nachdem die Stelle des Branch Chief der German Administration of Justice (später auch Legal Advice and Legislation) Branch lange Zeit unbesetzt gewesen war, hatte Ernst Anspach 1948/49 diese Stelle inne. Er war zunächst Deputy Chief der Branch gewesen. Die Prisons Branch leitete von 1947 bis zum Ende Wallace Lawrence. Die Leitung der MG Courts Branch, die ebenfalls lange unbesetzt war, hatte von 1947 bis zu ihrem Wegfall Dewitt White inne, der zunächst Chief des Field Teams in Wiesbaden gewesen war.

\section{Liaison and Security Control Division / Office of Superviser of Detachments - Liaison and Security Offices}

\section{a) Aufgaben}

In den ersten Monaten der Besetzung hielt das Office of Superviser of Detachments, das mit drei Offizieren ausgestattet war, die Verbindung zwischen den Detachments und dem Hauptquartier (später OMGHE) aufrecht. Zu seinen Aufgaben gehörte die Betreuung der Detachments bei den ersten Bestandsaufnahmen und bei der Herstellung von geregelten Beziehungen zu den taktischen Einheiten. Nicht Kontrolle, sondern Hilfe und Unterstützung standen im Vordergrund der Arbeit. So wurden in der Anfangsphase die Reisen des Superviser nicht als Inspektionsreisen, sondern als Besuche deklariert. Allerdings hatte der erfolgreich tätige Superviser, wie der erste Historical Report vermerkte, in der ersten Zeit keine Weisungsbefugnis. Das änderte sich, als nach der Auflösung der Detachments und der Errichtung der Liaison \& Security Offices das Office of the Superviser zur Liaison \& Security Control Section (LSC) wurde. ${ }^{186}$ Die Section unterstand zunächst dem Deputy Director for Operations und - nachdem eine Deputy Director Stelle entfallen war - dem Executive Officer. Ihre wesentlichen Aufgaben bestanden in der Einführung, Organisation und Durchführung regelmäßiger Fortbildungsseminare für die Liaison \& Security Officers sowie in den häufig durchgeführten regelmäßigen Inspektionsreisen zu den einzelnen Liaison \& Security Offices.

186 8/123-2/13. OMGHE: Historical Report October 1945 gibt für den gleichen Zeitpunkt die Errichtung der Liaison \& Security Control Division an. 
Wie bei vielen Divisions war auch bei der Liaison \& Security Control Division die Bezeichnung nie einheitlich geregelt: Im internen Dienstbetrieb ohnehin nur "Liaison \& Security Control“ (LSC) genannt, finden sich daneben sowohl „Liaison \& Security Section" als auch „Liaison \& Security Control Office“. Selbst nach der Errichtung der Liaison \& Security Division Anfang 1948 tauchten bis weit ins Frühjahr hinein noch Organisationspläne auf, die die Bezeichnung „Section“ verwendeten. ${ }^{187}$

\section{b) Organisation}

Der Organisationsaufbau von Liaison \& Security Control blieb vom Juni 1946 bis Ende 1948 unverändert und bot folgendes Bild: ${ }^{188}$

Ubersicht 44: Organisation der Liaison and Security Control Division, OMGHE, 1. Januar 1948

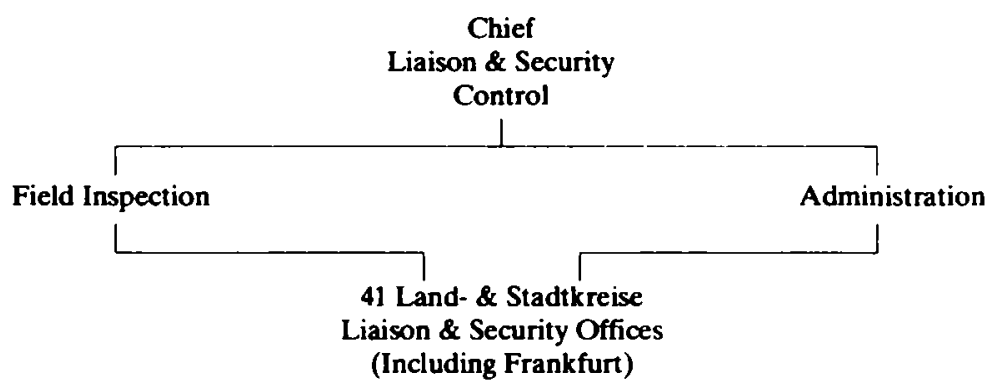

Im Laufe des Jahres 1948 entfalteten die Liaison \& Security Offices zunehmend Aktivităten im Rahmen des Reorientation Program und unterstützten die Armee bei ihren Youth Activities. Beide Initiativen zielten auf die Einübung parlamentarisch-demokratischer Verhaltensweisen und -normen ab. Während sich das Reorientation Program vorwiegend an Erwachsene richtete und durch öffentliche Diskussionsveranstaltungen, Filmvorführungen, Kulturaustausch usw. versuchte, seine Ziele zu erreichen, konzentrierte sich die Armee auf die Unterstützung von verschiedenen Sparten im Jugendbereich. ${ }^{189}$

Diese neuen Arbeitsschwerpunkte hatten zur Folge, daB nun die Division in vier Branches gegliedert wurde. Die Verwaltungsabteilung entfiel und die Aufgabe der Field Inspections Branch übernahm die Liaison with Posts Branch. Eine eigene Reports Branch war für die monatlichen Reorientation Reports zuständig und berichtete regelmäßig über die Einhaltung der Sicherheitsvorschriften für die LSOs. Implementation to Liaison \& Security Offices Branch organisierte monatliche und zweimonat liche Weiterbildungsseminare für die Liaison \& Security Officers und erstellte Arbeitshilfen für deren Büros. Die Policies on Reorientation Branch war für die demokratische Erziehung der Deutschen sowie die Herstellung guter Beziehungen zwischen Militärregierung, Militärstandorten und deutscher Bevölkerung zuständig.

Durch diese Umorganisation hatte die Liaison \& Security Control Division von Ende 1948 bis zu ihrer Auflösung folgendes Aussehen: ${ }^{190}$

187 Viele Beispiele in 8/216-2/9; siehe auch 5/41-3/10 und 5/41-2/17.

188 5/41-3/10.

${ }^{189} \mathrm{Vgl}$. OMGUS-Hb, Teil Hessen B.7.

190 5/41-3/10. 
Ubersicht 45: Organisation der Liaison and Security Control Division, OMGHE, 31. Dezember 1948

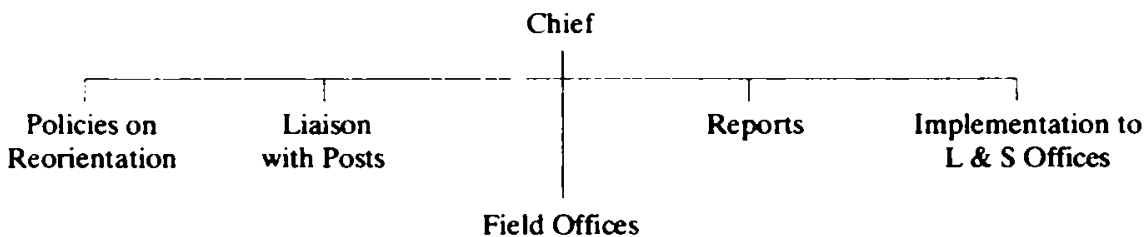

\section{c) Personal}

Die Liaison \& Security Control Division war stets eine der kleinsten Divisions innerhalb von OMGHE. Bis zum Juni 1946 fungierte der Deputy Director Howard R. Cress als Superviser of Detachments, unterstutzt von seinem Stellvertreter William E. Lockwood. Bei der Einrichtung der Liaison \& Security Section im Juni 1946 war eine Sollstärke von 22 Offizieren vorgesehen, die jedoch nie erreicht wurde. ${ }^{191}$ Am 1 . Juli 1946 versahen neun Offiziere und acht Mannschaftsdienstgrade ihren Dienst bei Liaison \& Security Control. Die Zahl der Mitarbeiter sank über das Jahr 1947 kontinuierlich ab. Am 1.Januar 1948 bestand die Division nur noch aus zwei Offizieren und zwei deutschen Angestellten.

Von diesem Zeitpunkt bis zur Auflösung der Division waren nie mehr als vier Offiziere, ein Soldat und zwei deutsche Angestellte tätig. Die Chiefs der Liaison \& Security Control Section waren in Personalunion gleichzeitig als Inspector General ${ }^{192}$ eine Art Beschwerdestelle, in etwa vergleichbar mit dem Wehrbeauftragten des Deutschen Bundestages.

Vom Sommer 1946 bis zum Juli 1947 leitete Lt.Col. Eugene M. Lee die Liaison \& Security Control Section. Abgelöst wurde er von seinem Vorgänger, Lt.Col. Howard R. Cress. Zu dessen Nachfolger wurde im Mai 1948 der inzwischen als Zivilist tătige Arsen L. Yakoubian ${ }^{193}$ ernannt, dessen offizielle Bestätigung allerdings erst im Dezember 1948 erfolgte.

Die Gesamtzahlen des Personals für den Bereich der Liaison \& Security Offices (mit Control Section) nahmen folgende Entwicklung:

Ubersicht 46: Anzahl der bei LSO (mit Control Section), OMGHE, Beschäftigten, Juli 1946 - Oktober 1948

$\begin{array}{llc}\text { Zeitpunkt } & \text { US-Personal } & \text { Deutsche } \\ \text { 1.Juli } 1946 & 193 & 1073 \\ \text { 1.Januar } 1947 & 181 & 1500 \\ \text { 15. März } 1948 & 132 & 837 \\ \text { 30. Oktober } 1948 & 76 & \mathbf{8 8 8}\end{array}$

Für 1949 lagen keine Zahlen vor.

191 OMGHE: Historical Report October 1945 - June 1946.

192 8/157-2/13;8/216-1/9.

193 Arsen L. Yakoubian, der während der gesamten Militärregierungszeit in den verschiedensten Positionen innerhalb von OMGHE gearbeitet hat, promovierte übrigens 1951 in New York mit einer Arbeit über Western Allied Occupation Policies and Development of German Democracy 1945 - 1951. 


\section{d) Die Liaison and Security Offices}

Nachdem bereits zum Jahresende 1945 und Anfang 1946 den lokalen und Regierungsbezirks-Detachments wesentliche Befugnisse entzogen und an deutsche Behörden übergeben worden waren ${ }^{194}$ und die Politik der Militärregierung mehr und mehr von direkten Eingriffen zur Überwachung deutscher Behörden überging, waren organisatorische Konsequenzen für das Detachment-System unausweichlich.

Auf Schwierigkeiten und Probleme, die im Zusammenhang mit einigen bereits eingerichteten Liaison \& Security Detachments auftraten, verweist ein Memorandum des Detachments Superviser Howard R. Cress an James R. Newman vom 27. März 1946. ${ }^{195}$ Es ging darin um die Liaison \& Security Offices, die als Unterabteilung von Kreis-Detachments (Parent Detachments) seit Anfang 1946 in jenen Landkreisen arbeiteten, die nicht mehr von einem „eigenen“ Kreis-Detachment betreut wurden. Cress beschrieb in seinem Bericht vor allem Probleme der Kompetenzabgrenzung und der Dienstwege. Weiter ging es darum, wie sich die bereits existierenden Liaison \& Security Offices auf die geplante Neuorganisation im Juni vorbereiten sollten.

Eine ausführliche Begründung der geplanten Neuorganisation, die die Verwaltung vereinfachen, Zeit, Arbeitskraft und Benzin einsparen sollte, hatte James R. Newman bereits im Februar 1946 in einer Denkschrift an General Lucius D. Clay gegeben. ${ }^{1 \%}$ Im einzelnen bedeutete die dann im Juni 1946 durchgeführte Reform:

- die Auflösung der Regierungsbezirks-Detachments und aller verbliebenen lokalen Detachments; ${ }^{197}$

- die Errichtung von Liaison \& Security Offices in allen Kreisstädten; ${ }^{198}$

- die Unterstellung der Liaison \& Security Offices unter OMGGH;

- die Reduzierung der Aufgaben der Liaison \& Security Offices auf Entnazifizierung, öffentliche Sicherheit, Berichterstattung und Verbindungsaufgaben sowohl mit deutschen Behörden als auch mit dem amerikanischen Militär und die Betreuung der Military Government Courts.

Damit war die exekutive Gewalt weitgehend bei OMGGH zusammengefaßt.

Ubersicht 47: Stellenbesetzung der Liaison and Security Offices, OMGHE, Juni 1946

Zuständigkeitsbereich

SK Frankfurt

SK Wiesbaden

LK Wetzlar

LK Dill

LK Gelnhausen

LK Biedenkopf

SK/LK Hanau

LK Oberlahn

LK Limburg

LK Maintaunus

LK Rheingau

LK Obertaunus

Standort
Frankfurt
Wiesbaden
Wetzlar
Dillenburg
Gelnhausen
Biedenkopf
Hanau
Weilburg
Limburg
Hofheim
Rüdesheim
Bad Homburg

Kommandierender Offizier

Col. Robert K. Phelps

Maj. Matthew F. Chotas

Capt. Mervin S. Clark

Capt. E. G. Stolper

Capt. J.G. Bennas

Capt. T. E. Faircloth

Maj. E. J. Emerick

Capt. A.G. Volz

Capt. P. H. Olsen

Maj. John C. Nelson

Capt. W. F. Hintz

Capt. Lawrence F. Jones

\footnotetext{
$194 \mathrm{Vgl}$. OMGUS-Hb, Teil Hessen A.5.

195 OMGHE: Historical Report October 1945 - June 1946, Appendix I (5/41-1/20).

${ }^{196}$ Ebenda.

197 General Order Nr. 14, OMGHE, 28.5.1946 (ebenda).

198 General Order Nr. 13, OMGHE. 21.5.1946 (ebenda).
} 


LK Usingen
LK Untertaunus
LK Schlüchtern
SK/LK Kassel
LK Melsungen
LK Fritzlar-Homberg
LK Ziegenhain
SK/LK Marburg
LK/SK Fulda
LK Hünfeld
LK Waldeck
LK Frankenberg
LK Eschwege
LK Witzenhausen
LK Hersfeld
LK Rotenburg
LK Hofgeismar
LK Wolfhagen
LK/SK Darmstadt
LK Groß-Gerau
SK/LK Offenbach
LK Bergstraße
LK Erbach
LK Büdingen
LK Dieburg
LK Friedberg
SK/LK GieBen
LK Lauterbach
LK Alsfeld

Usingen
Bad Schwalbach
Schlüchtern
Kassel
Melsungen
Fritzlar
Ziegenhain
Marburg
Fulda
Hünfeld
Korbach
Frankenberg
Eschwege
Witzenhausen
Hersfeld
Rotenburg
Hofgeismar
Wolfhagen
Darmstadt
GroB-Gerau
Offenbach
Heppenheim
Erbach
Büdingen
Dieburg
Friedberg
GieBen
Lauterbach
Alsfeld

\author{
Capt. R.F. Gibney \\ Capt. Thomas W. Harris \\ Capt. S. B. Borda \\ Lt.Col. William R. Swarm \\ Maj. W.C. Gipple \\ Capt. G.D. Fexy \\ Capt. R. B. Elwell \\ Lt.Col. L. G. Kelly \\ Lt.Col. Howard R. Cress \\ Capt. E. T. Tedick \\ Capt. D. W. Shea \\ Maj. Lewis S. Williams \\ Capt. J. F. Philp \\ Maj. Lawrence H. Brown \\ Maj. Michael Baymor \\ Capt. G. W. Davis \\ Capt. Leonard R. Allen \\ Capt. Stanley A. Karas \\ Lt.Col. Arthur Skarry \\ Capt. H. L. Edberg \\ Capt. I. Boncowitz \\ Maj. Robert A. Gish \\ Capt. R. O. Didlo \\ Capt. N. V. Steenberg \\ Capt. John S. Chapin \\ Capt. Cecil S. Parshall \\ Maj. Charles F. Russe \\ Capt. H. Nickelsberg \\ Capt. H. B. Miller
}

Die Organisation, die Aufgaben, die Verwaltung und das Berichtswesen der Liaison \& Security Offices und die Pflichten der dort tätigen Offiziere wurden von OMGUS durch ausführliche Standing Operating Procedures (SOP) beschrieben. ${ }^{199}$ Ein Annex to SOP for Liaison \& Security Offices, der von OMGGH in Abstimmung mit den einzelnen Divisions entwickelt worden war, gab den Liaison \& Security Offices zusätzliche Erläuterungen und Handreichungen für ihre Arbeit.

Als „Augen und Ohren der Landesmilitärregierung“ und als „persönliche Repräsentanten der Vereinigten Staaten und ihrer Militärregierung in Deutschland und als Beispiele demokratischer Kultur" ${ }^{200}$ waren die Liaison \& Security Offices und ihre Mitarbeiter hohen Verhaltenserwartungen ausgesetzt. Zur besseren Koordination der Arbeit wurden ab Juli 1946 monatliche Treffen der Senior Liaison \& Security Officers im Wiesbadener Landeshaus durchgeführt. Die Hauptprobleme der LSOs waren die Entnazifizierung und die wachsenden Raum- und Platzanforderungen der stetig nicht zuletzt durch Familiennachzug - größer werdenden Militärstandorte. Erschwerend kam hinzu, daß sich durch den raschen Abzug der taktischen Truppen die Notwendigkeit ergeben hatte, eine mobile Eingreiftruppe für Polizei- und Bewachungsaufgaben, die Constabulary, aufzustellen. Bewährte Verbindungen zur Armee waren dadurch unterbrochen, völlig neue Verbindungen mußten aufgebaut werden. ${ }^{201}$ Weitere Schwierigkeiten bereiteten der chronische Personalmangel, der Weggang erfahre-

$1998 / 213-2 / 11 ; 8 / 213-2 / 15 ; 5 / 3546-1 / 4$.

$2008 / 213-2 / 11$.

201 OMGHE: Historical Report July - December 1946 ; auch in 8/213-2/13. 
ner und ausgebildeter Offiziere und die turnusmäßig durchgeführten Personalrotationen. Offiziere und Mannschaften sollten nicht länger als ein Jahr an einem Standort bleiben.

Ubersicht 48: Stellenbesetzung der Liaison and Security Offices, OMGHE, 1. April $1947^{202}$

\begin{tabular}{|c|c|c|}
\hline Zuständigkeitsbereich & Standort & Kommandierender Offizier \\
\hline LK Alsfeld & Alsfeld & Capt. Stanley A. Karas \\
\hline LK Bergstraße & Heppenheim & Maj. Arlo R. Gill \\
\hline LK Biedenkopf & Biedenkopf & Capt. William L. Culbertson \\
\hline LK Büdingen & Büdingen & Capt. George P. Johnson \\
\hline SK Darmstadt & Damstadt & Li.Col. Arthur Skarry \\
\hline LK Dieburg & Dieburg & Capt. Mervin S. Clark \\
\hline LK Dill & Dillenburg & Maj. Ben. L. Bassinor \\
\hline LK Eschwege & Eschwege & Maj. George P. Moore \\
\hline LK Erbach & Erbach & Maj. Lawrence H. Brown \\
\hline LK Frankenberg & Frankenberg & Maj. Lewis S. Williams \\
\hline SK Frankfurt & Frankfurt & Maj. Gerald C. Sola \\
\hline LK Friedberg & Friedberg & Maj. Porter E. Perry \\
\hline LK Fritzlar-Homberg & Fritzlar & Maj. John Connely \\
\hline SK Fulda & Fulda & Lt.Col. Howard R. Cress \\
\hline LK Gelnhausen & Gelnhausen & Capt. George D. Fexy \\
\hline SK Gießen & GieBen & Maj. Charles F. Russe \\
\hline LK Groß-Gerau & Groß-Gerau & Capt. Norman L. Neider \\
\hline SK Hanau & Hanau & Maj. Robert A. Gish \\
\hline LK Hersfeld & Hersfeld & Maj. Michael Baymor \\
\hline LK Hofgeismar & Hofgeismar & Capt. Leonard R. Allen \\
\hline LK Hünfeld & Hünfeld & Capt. William G. Westbrook \\
\hline SK Kassel & Kassel & Lt.Col. Gustav J. Albrecht \\
\hline LK Lauterbach & Lauterbach & Capt. Clyde R. Argo \\
\hline LK Limburg & Limburg & Capt. John S. Chapin \\
\hline LK Maintaunus & Hofheim & Maj. John C. Nelson \\
\hline SK Marburg & Marburg & Lt.Col. Charles H. Reed \\
\hline LK Melsungen & Melsungen & Maj. Will. C. Gipple \\
\hline LK Oberlahn & Weilburg & Lt.Col. Merle A. Meacham \\
\hline LK Obertaunus & Bad Homburg & Capt. J. Nottingham \\
\hline SK Offenbach & Offenbach & Lt.Col. John C. Rose \\
\hline LK Rheingau & Rüdesheim & Capt. James R. Hyde \\
\hline LK Rotenburg & Rotenburg & Capt. William F. Hintz \\
\hline LK Schlüchtern & Schlüchtern & Capt. Oscar F. Howard \\
\hline LK Untertaunus & Bad Schwalbach & Maj. Mattbew F. Chotas \\
\hline LK Usingen & Usingen & Capt. James W. Francis \\
\hline LK Waldeck & Korbach & Maj. Cecil S. Parshall \\
\hline LK Wetzlar & Wetzlar & Maj. David M. Easterday \\
\hline SK Wiesbaden & Wiesbaden & Col. Francis H. Boucher \\
\hline LK Witzenhausen & Witzenbausen & Maj. Everett E. Covell \\
\hline LK Wolfhagen & Wolfhagen & Capt. Thomas E. Faircloth \\
\hline LK Ziegenhain & Ziegenhain & Capt. Thomas W. Harris \\
\hline
\end{tabular}

Eine einschneidende Veränderung in der Organisation der Liaison \& Security Offices kündigte Ende 1947 die Aufforderung von OMGUS an OMGHE an, Pläne für eine

$2025 / 41-2 / 5$. 
fünfzigprozentige Reduzierung des Liaison \& Security Personals vorzulegen. ${ }^{203}$ Der daraufhin von OMGHE entwickelte Organisationsplan sah nun anstatt der 41 KreisLiaison \& Security Offices die Einrichtung von 14 Districts mit Nebenstellen vor. ${ }^{204}$

Der weitere Verlauf der Umorganisation zeigt deutlich den inzwischen erreichten „Bürokratisierungsgrad“ der Militärregierung: Ein Memorandum vom 11. Juni 1948205 ordnete zum 14. Juni 1948 die Errichtung von zwölf Liaison \& Security Districts an und wurde mit Memorandum vom 9. August $1948^{206}$ um einen dreizehnten District erweitert. Beide Memoranda wurden mit einem Memorandum vom 1.November $1948^{207}$ für nichtig erklärt, das seinerseits die Einrichtung von 16 Liaison \& Security Districts vorsah. Eine endgültige Klärung brachte schließlich das Memorandum vom 28. Dezember 1948, das wiederum das vorherige Memorandum für nichtig erklärte und die Errichtung von nunmehr zehn Liaison \& Security Districts zum 10. Januar 1949 anordnete.

Ubersicht 49: District Liaison and Security Offices mit Nebenstellen, OMGHE, 10. Januar 1949

a) Wiesbaden District Liaison \& Security Office

Headquarters in Wiesbaden

Sub-Office Maintaunus

Sub-Office Untertaunus

Sub-Office Rheingau

Sub-Office Limburg

b) Frankfurt District Liaison \& Security Office

Headquarters in Frankfurt

Sub-Office Offenbach

Sub-Office Maintaunus

Sub-Office Friedberg

Sub-Office Usingen

c) Darmstadt District Liaison \& Security Office

Headquarters in Darmstadt

Sub-Office Grob-Gerau

Sub-Office BergstraBe

Sub-Office Dieburg

Sub-Office Erbach

d) Hanau District Liaison \& Security Office

Headquarters in Hanau

Sub-Office Büdingen

Sub-Office Gelnhausen

Sub-Office Schlüchtern

e) Wetzlar District Liaison \& Security Office

Headquarters in Wetzlar

Sub-Office Oberiahn

Sub-Office Dillkreis

${ }^{203}$ Brief vom 26.12.1947 (8/216-2/10).

204 Ebenda.

$2058 / 213-2 / 15$.

206 8/157-2/13.

${ }^{207}$ Ebenda und 8/190-1/2. 
f) Gießen District Liaison \& Security Office

Headquarters in Gießen

Sub-Office Alsfeld

Sub-Office Lauterbach

g) Marburg District Liaison \& Security Office

Headquarters in Marburg

Sub-Office Biedenkopf

Sub-Office Frankenberg

h) Fulda District Liaison \& Security Office

Headquarters in Fulda

Sub-Office Hünfeld

Sub-Office Hersfeld

Sub-Office Rotenburg

i) Fritzlar-Homberg District Liaison \& Security Office

Headquarters in Fritzlar

Sub-Office Waldeck

Sub-Office Melsungen

Sub-Office Ziegenhain

j) Kassel District Liaison \& Security Office

Headquarters in Kassel

Sub-Office Wolthagen

Sub-Office Hofgeismar

Sub-Office Witzenhausen

Nach der Auflösung von OMGUS und seiner Länder-Militärregierungen im September 1949 fungierte das Office of the United States High Commissioner for Germany als amerikanischer Teil der Alliierten Hochkommission. In Hessen trat an die Stelle von OMGHE das Office of Land Commissioner for Hesse. Die bisherigen Liaison \& Security Offices wurden Area Offices der nunmehr nur noch sechs Districts. Die Liaison \& Security Officers hießen von nun an United States Resident Officers. ${ }^{208}$

Ubersicht 50: US Resident Offices in Hessen, 1. Oktober 1949

Organisationsplan für 1. Oktober 1949

I FRANKFURT DISTRICT
(a) Friedberg-Usingen Area
(b) Hanau Area
(c) Büdingen Area
(d) Obertaunus Area

II WIESBADEN DISTRICT
(a) Rheingau-Untertaunus Area
(b) Limburg Area
(c) Maintaunus Area

III DARMSTADT DISTRICT
(a) Groß-Gerau Area
(b) Bergstraße Area
(c) Dieburg-Erbach Area
(d) Offenbach Area

$2088 / 213-2 / 15$ 
IV KASSEL DISTRICT (Hofgeismar)

(a) Waldeck-Wolfhagen Area

(b) Witzenhausen Area

(c) Eschwege Area

(d) Fritzlar Area

V MARBURG DISTRICT

(a) Frankenberg-Biedenkopf Area

(b) Gießen Area

(c) Wetzlar Area

(d) Oberlahn-Dillkreis Area

VI HERSFELD DISTRICT(Hünfeld)

(a) Ziegenhain Area

(b) Fulda Area

(c) Alsfeld-Lauterbach Area

(d) Melsungen-Rotenburg Area

Ubersicht 51: Stellenbesetzung der Liaison and Security Offices, OMGHE, März $1948^{209}$

Zuständigkeitsbereich

LK Alsfeld

LK Bergstraße

LK Biedenkopf

LK Büdingen

SK/LK Darmstadt

LK Dieburg

LK Dill

LK Erbach

LK Eschwege

LK Frankenberg

SK Frankfurt

LK Friedberg

LK Fritzlar-Homberg

SK/LK Fulda

LK Gelnhausen

SK/LK GieBen

LK Groß-Gerau

SK/LK Hanau

LK Hersfeld

LK Hofgeismar

LK Hünfeld

SK/LK Kassel

SK Lauterbach

LK Limburg

LK Maintaunus

SK/LK Marburg

LK Melsungen

LK Oberlahn

LK Obertaunus

SK/LK Offenbach

LK Rheingau
Standort

Alsfeld

Heppenheim

Biedenkopf

Büdingen

Darmstadt

Dieburg

Dillenburg

Erbach

Eschwege

Frankenberg

Frankfurt

Friedberg

Fritzlar

Fulda

Gelnhausen

Gießen

Groß-Gerau

Hanau

Hersfeld

Hofgeismar

Hünfeld

Kassel

Lauterbach

Limburg

Hotheim

Marburg

Melsungen

Weilburg

Bad Homburg

Offenbach

Rüdesheim
Kommandierender Offizier

1st Lt. E.C. Stam

Maj. D.J. Heffernan

Capt. William L. Culbertson

Capt. T.L. Vitulio

Lt.Col. J.C. Rose

Capt. Mervin S. Clark

Capt. Richard W. Smith

Capt. J. R. Hyde

Maj. E. J. Hart

Capt. G.P. Johnson

Maj. Gerald C. Sola

Maj. H.P. Radigan

Maj. W.C. Gipple

Maj. T. L. Tempest

Capt. G.D. Fexy

Maj. George P. Moore

Capt. W.M. Condon

Lt.Col. C. H. Reed

Maj. W.J. Salwocki

Capt. W.S. Chadwick

1st Lt. A. Abraham

Lt.Col. G.J. Albrecht

Capt. C. R. Argo

J.S. Sabol

Maj. John C. Nelson

Maj. H.S. Homecker

Capt. C.S. Bechel

Capt. Oscar F. Howard

Maj. C. A. Vollrath

Maj. W.R. Sheehan

Capt. W.L. Young

209 8/216-2/9. 


$\begin{array}{ll}\text { LK Rotenburg } & \text { Rotenburg } \\ \text { LK Schlüchtern } & \text { Schlüchtem } \\ \text { LK Untertaunus } & \text { Bad Schwalbach } \\ \text { LK Usingen } & \text { Usingen } \\ \text { LK Waldeck } & \text { Korbach } \\ \text { LK Wetzlar } & \text { Wetzlar } \\ \text { SK Wiesbaden } & \text { Wiesbaden } \\ \text { LK Witzenhausen } & \text { Witzenhausen } \\ \text { LK Wolfhagen } & \text { Wolfhagen } \\ \text { LK Ziegenhain } & \text { Ziegenhain }\end{array}$

Capt. R. J. Fitzsimmons

Capt. S. Kershaw

Maj. Matthew F. Chotas

Capt. H.J. Wills

Maj. P.E. Perry

Maj. Charles F. Russe

Col. Francis $\mathrm{H}$. Boucher

Maj. B. L. Bassinor

Capt. V.K. Ledbetter

Capt. John S. Chapin

Ubersicht 52: Stellenbesetzung der Liaison and Security Offices, Juli $1949^{210}$

Zuständigkeitsbereich
SK/LK Darmstadt
LK GroB-Gerau
LK BergstraBe
LK Dieburg
LK Erbach
SK Frankfurt
SK/LK Offenbach
LK Obertaunus
LK Friedberg
LK Usingen
LK Fritzlar-Homberg
LK Waldeck
LK Melsungen
LK Ziegenhain
SK/LK Fulda
LK Hünfeld
LK Hersfeld
LK Rotenburg
SK/LK Gießen
LK Alsfeld
LK Lauterbach
SK/LK Hanau
LK Büdingen
LK Gelnhausen
LK Schlüchtern
SK/LK Kassel
LK Eschwege
LK Witzenhausen
LK Hofgeismar
LK Wolfhagen
SK/LK Marburg
LK Biedenkopf
LK Frankenberg
LK Wetzlar
LK Dill
LK Oberlahn
SK Wiesbaden
LK Limburg
LK Maintaunus

Zuständigkeitsbereich

Standort

Darmstadt

GroB-Gerau

Heppenheim

Dieburg

Erbach

Frankfurt

Offenbach

Bad Homburg

Friedberg

Usingen

Fritzlar

Korbach

Melsungen

Ziegenhain

Fulda

Hünfeld

Hersfeld

Rotenburg

GieBen

Alsfeld

Lauterbach

Hanau

Büdingen

Gelnhausen

Schlüchtem

Kassel

Eschwege

Witzenhausen

Hofgeismar

Wolfhagen

Marburg

Biedenkopf

Frankenberg

Wetzlar

Dillenburg

Weilburg

Wiesbaden

Limburg

Hofheim
Kommandierender Offizier

H.P. Radigan

nicht besetzt

nicht besetzt

nicht besetzt

nicht besetzt

Gerald C. Sola

W. R. Sheehan

F.N. Grove

J.W. Jergensen

nicht besetzt

P.E. Perry

nicht besetzt

B. Zemplany

nicht besetzt

Mervin S. Clark

W.L. Burns

F.J. Green

J.F. Keough

G.P. Moore

W.J. Hoffman

nicht besetzt

R.O. Didlo

nicht besetzt

J. A. Goodnight

nicht besetzt

Robert A. Goetcheus

N.G. Turner

J.E. Monoghan

nicht besetzt

nicht besetzt

E. L. Weyland

nicht besetzt

nicht besetzt

C.E. Lloyd

nicht besetzt

nicht besetzt

G. E. Vadney

J.S. Huffner

nicht besetzt

$2105 / 42-3 / 11$. 
LK Rheingau

LK Untertaunus
Rüdesheim

Bad Schwalbach nicht besetzt

nicht besetzt

\section{Manpower Division}

\section{a) Aufgaben}

Die Manpower Division war zuständig für Arbeitsbedingungen, Arbeitsverwaltung, Sozialversicherung, Tariffragen und Löhne. In ihre Zuständigkeit fielen auch die Gewerkschaften.

Diese Aufgabenbereiche wurden zunächst auf Detachment-Ebene bearbeitet. Jedes Kreis-Detachment verfügte uber einen Kreis Labor Officer, der Berichte an die Regierungsbezirks-Detachments abgab und auch für die Zulassung der Gewerkschaften zuständig war. Zum 1. Januar 1946 entfielen diese Zuständigkeiten. Die Manpower Division nahm jetzt alle Kontrollfunktionen über das hessische Ministerium für Arbeit und Wohlfahrt wahr, das am 14. Oktober 1945 auf Grundlage von Organizational Direction Nr. 2, OMGHE, gebildet worden war.

Die Gewerkschaftsgrindungen vollzogen sich zunächst auf Orts- und Kreisebene. ${ }^{211}$ Die erste Gewerkschaft im Regierungsbezirk Wiesbaden bildete sich am 29. August 1945 in Hanau. ${ }^{212}$ Bis Juni 1946 bestanden in 33 Landkreisen Gewerkschaften, landesweit waren sechs Einzelgewerkschaften organisiert. ${ }^{213}$ Der erste allgemeine landesweite GewerkschaftskongreB fand am 24/25. August 1946 in Frankfurt statt. ${ }^{214}$

Bereits im Mai 1945 hatte das Landesarbeitsamt in Frankfurt seine Arbeit wieder aufgenommen. Im Oktober wurde dem Amt die Aufsicht uber die 14 inzwischen wieder eingerichteten regionalen Arbeitsämter zugesprochen. Eines der Hauptprobleme, mit dem die Arbeitsverwaltung konfrontiert war, lag im hohen Personalbedarf der amerikanischen Streitkräfte. Hatten im Oktober 1945 noch 26839 Deutsche bei den Truppen gearbeitet, so waren es im Mai 1946 bereits 63786.

Die Manpower Division setzte auf den Aufbau von Industriegewerkschaften, um die Demokratisierung in Verbänden und Betrieben zu fördern. In den Auseinandersetzungen um Sozialisierung und Betriebsrătegesetz bezog die Division von sich aus keine klare Position. Aktiv unterstützte sie den Versuch, in Hessen Freiwillige für den Ruhrbergbau zu werben.

b) Organisation

Die Manpower Division war bis zu ihrer Auflösung am 20. April 1948 in fünf Branches gegliedert:

- Labor Relations (industrielle Arbeitsbeziehungen) war für Gewerkschaften und Betriebsräte zuständig.

- Wages, Hours, Labor Standards beschäftigte sich mit Löhnen, Arbeitszeit und Arbeitsverhältnissen. Sie überwachte die Einhaltung von Vorgaben der Militärregierung und analysierte Tarifabschlusse.

- Social Insurance kontrollierte das Sozialversicherungswesen.

211 OMGHE: Historical Report October 1945 - June 1946.

212 Chronology, S.3.

213 OMGHE: Historical Report October 1945 - June 1946.

214 Chronology, S. 26. 
- Labor Allocation hielt Kontakt mit den anderen Divisions und war Ansprechpartner der US-Armee für die Zuweisung von Arbeitskräften. Die Branch hatte auch die Aufsicht über die Arbeitsämter.

- Housing kontrollierte die örtlichen Wohnungsämter und arbeitete mit der Building Material Section, Economics Divison, zusammen.

Die Organisation der Manpower Division stellte sich im Juni 1946 wie folgt dar:2is

Übersicht 53: Organisation der Manpower Division, OMGHE, 30. Juni 1946

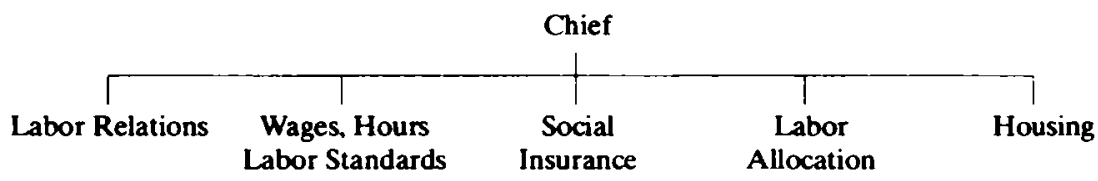

Da die Division aber nie mehr als vier amerikanische Mitarbeiter zählte - die meiste Zeit waren nur zwei Stellen besetzt -, hatte diese Organisationsgliederung wohl mehr selbstdarstellerischen Charakter.

Seit dem 20. April 1948 nahm die Manpower Branch in der neu gebildeten Bipartite Liaison Divison (später in der Economics Division) die Aufgaben der früheren Manpower Division wahr. Am 22. April 1948 wurde der bisherigen Division Chief und neue Branch Chief zum Manpower Adviser des Direktors von OMGHE ernannt.

\section{c) Personal}

Die Manpower Division gehörte zu den kleinsten Divisions von OMGHE. Im Vergleich beispielsweise zur Economics Division nimmt sie sich geradezu zwergenhaft aus. In den Jahren 1946/47 verfügte die Manpower Division zumeist über vier amerikanische Mitarbeiter (zum 1. Januar 1947 vorubergehend nur drei). Seit 1. Januar 1948 arbeiteten nur noch drei Amerikaner mit. In der Manpower Branch der Bipartite Liaison Division waren zunächst zwei Amerikaner aus der aufgelösten Manpower Division tätig. 1949 blieb nur noch der Branch Chief übrig.

Die Manpower Division zeichnet sich durch eine bemerkenswerte personelle Stabilität aus. Glenn E. Garrett war seit September 1944 im Manpower-Bereich tätig gewesen und leitete die Division von ihrer Bildung bis zu ihrer Auflösung. Danach war er Chief der Manpower Branch in der Bipartite Liaison Division und Manpower Adviser von J. R. Newman. Ihm zur Seite stand Albert A. Maurinac, der Ende 1947 für längere Zeit in den USA weilte und Mitte 1948 dann endgültig ausschied. Am 20. September 1947 kam Evan T. Hopkins zur Division, der für Arbeitsverwaltung zuständig war und in der zweiten Jahreshälfte 1948 ausschied. Ab Ende 1947 arbeitete vorübergehend Abraham Bluestone als Military Government Trainee - eine Art Militärregierungsreferendar - in der Division.

\section{Post, Telephone and Telegraph Division}

Die amerikanischen Truppen legten beim Einmarsch alle Postverbindungen lahm, und die Army Signal Corps übernahmen die Kontrolle über die fernmeldetechnischen Anlagen. Nachdem die Detachments ihre Arbeit aufgenommen hatten, be-

$2155 / 41-27$. 
nannten sie jeweils einen verantwortlichen Post Officer, der für die deutsche Post in seinem Kreis zuständig war. Ausschließlich für Postangelegenheiten zuständige Offiziere saßen in Frankfurt, Darmstadt, Wiesbaden und Kassel. Zum 29. Oktober 1945 wurden die Detachments von der Kontrolle über die Post entbunden, nur die beiden Postoffiziere in Frankfurt und Wiesbaden behielten ihre Verantwortungsbereiche. Zum 1.Februar 1946 wurden dann alle Post Officers in der Post, Telephone and Telegraph (PT\&T) Section von OMGHE in Wiesbaden zusammengefaßt. ${ }^{216}$ Die Section (später Division) hatte ihren Hauptsitz in Frankfurt, dem „Nervenzentrum“ des hessischen Postsystems. ${ }^{217}$ Sie hatten ihre Diensträume in der Frankfurter Oberpostdirektion bezogen, deren früherer Zuständigkeitsbereich ungefähr den Grenzen des neu geschaffenen Landes Hessen entsprach. Die Spitzen der Postdirektion waren im Juli 1945 vom Detachment E-6 (Frankfurt) ernannt und in den folgenden Monaten kontrolliert worden. Am 1. Oktober 1945 übernahm E-2 in Marburg formal die Zuständigkeit, die dann mit Auflösung von E-2 endgültig an E-5/OMGHE in Wiesbaden überging.

Bis zum 1.Oktober 1945 war es gelungen, in der gesamten US-Zone den Brief- und Telefonverkehr wieder in Gang zu bringen. Die Postsparkasse hatte ihre Arbeit schon im August 1945 wieder aufgenommen. Der Telegrammdienst war bis zum 1. Dezember 1945 wiederhergestellt. ${ }^{218}$ Die Entnazifizierung der Post lag zunächst in Händen der lokalen Detachments. Diese Zuständigkeit ging teilweise im Oktober 1945 und insgesamt im Januar 1946 an die Special Branch von OMGHE uber. Von den 18000 Bediensteten der Postdirektion muBten 3500 entlassen werden.

War zunächst jede Postdirektion für sich der Militärregierung gegenüber verantwortlich gewesen, so wurde zum 15. April 1946 in München eine Oberpostdirektion eingerichtet, die vorübergehend als Zentralstelle für die deutsche Post in der US-Zone fungierte und gegenüber OMGUS die Gesamtverantwortung trug. ${ }^{219}$ OMGUS zentralisierte deshalb auch die PT\&T-Arbeit der Militărregierung bei sich in Berlin. Die Post, Telephone and Telegraph Division von OMGHE gab zum 31. Dezember 1946 ihre Zuständigkeit an die Communications Branch von OMGUS ab und wurde aufgelöst. 220

Im Oktober 1945 arbeiteten 15 Offiziere und drei Soldaten im Post, Telephone and Telegraph-Bereich. Die Zahl der Offiziere verringerte sich zum 1.Februar 1946 auf acht. Ende Juni waren nur noch drei Offiziere der Section/Division zugeordnet, von denen zwei im Sommer 1946 den Zivilisten-Status annahmen. Gemeinsam mit den drei Amerikanern arbeiteten zehn Deutsche in der Division. Bis Dezember 1946 hatte sich die Zahl der Amerikaner auf zwei verringert.

Juli/August 1945 war Major William B. Campbell Chef des Post, Telephone and Telegraph-Bereichs. Von August bis Dezember 1945 nahm Lt.Col. Henry S. Ferguson diese Stelle ein. Im ganzen Jahr 1946 leitete James C. Paxton die Section/Division. Er hatte diese Leitungsposition für Hessen auch nach Eingliederung von Post, Telephone and Telegraph in OMGUS noch bis zum September 1947 inne.

216 OMGHE: Historical Report October 1945 - June 1946.

217 So der zeitweilige Division Chief James C. Paxton in einem Schreiben vom 3.1.1946 (8/2163/8).

218 OMGHE: Historical Report October 1945 - June 1946.

219 Ebenda.

${ }^{220}$ OMGHE: Historical Report July - December 1946. 


\section{Property Control Division (ab 20. April 1948: Property Division)}

a) Aufgaben

Die Property Control Division arbeitete auf der Grundlage des Militärregierungsgesetz Nr. 52 (Sperre und Kontrolle von Vermögen) und einer USFET Directive vom 7. Juli 1945. Danach fielen vor allem fünf Kategorien von Vermögen unter die Aufsicht der Militärregierung:

- Staatseigentum des früheren „Dritten Reiches“ und seiner Kriegsverbündeten;

- Eigentum der NSDAP und angeschlossener oder von ihr betreuter Organisationen;

- Eigentum, das Mitgliedstaaten der Vereinten Nationen, neutralen Ländern oder anderen auswärtigen Besitzern gehörte;

- Eigentum stark belasteter Nationalsozialisten;

- Eigentum, das während der NS-Herrschaft unter Zwang oder durch Einschüchterung den Besitzer gewechselt hatte. 221

Die Property Control-Arbeit sollte so zwei politischen Zielen dienen:

- einerseits solite sie im „regierungslosen“ Zustand unmittelbar nach der Besetzung staatliches und ausländisches Eigentum vor Schäden bewahren;

- andererseits sollte sie zweifelhafte Eigentumstransaktionen während der NS-Zeit rückgängig machen und als Strafmittel gegen NS-Verbrecher dienen. ${ }^{22}$

Früheres Eigentum der deutschen Wehrmacht fiel in die Zuständigkeit der USArmee. ${ }^{223}$ Es bestand jedoch „,beträchtliche Verwirrung“ darüber, wie mit Waren aus Armeebeständen verfahren werden sollte. Eine Anweisung des kommandierenden Generals der 7.US-Armee vom November 1945 bestimmte, daB Verkaufserlöse von Wehrmachtsgütern auf einem Sperrkonto der Militărregierung gutzuschreiben seien. Das Militärregierungsgesetz Nr.54 vom 27. August 1945 übergab landwirtschaftlich nutzbares Eigentum des Reiches, dessen Luftwaffe und einiger NS-Organisationen an die Länder in der US-Zone. 224

In den ersten Monaten nach der Besetzung waren die lokalen Detachments für die Vermögensverwaltung zuständig. Jedes Detachment hatte einen Property Control Officer. Am 16. Juli 1945 nahm die Property Control Section des Detachments E1A2 (E-5), aus dem dann die Property Control Division von OMGHE hervorging, ihre Arbeit auf. Am 21. Juli 1945 entzog eine USFET Directive den Detachments die ausschließliche Kompetenz über die Vermögenskontrolle. Die örtlichen Militärregierungen hatten jetzt ihre Erhebungen an den Deputy Chief Property Control Officer des Western Military District weiterzuleiten. Nach Bildung von OMGHE am 1.Oktober 1945 wurde die Registratur der Vermögenskontrolle in der Property Control Branch, Finance Division, OMGUS, zentralisiert.

Die Property Control Division und die Property Control Officers der Detachments hatten die Aufgabe, Vermögen aufzuspüren, das unter die Kontrolle der Militärregierung fiel. Die Kreis Special Branches und CIC stellten dazu ihre Informationen zur Verfügung. Zur Verwaltung größerer Vermögenswerte wurden Treuhänder eingesetzt, deren Arbeit die Property Control Division kontrollierte. Sie koordinierte auch

221 OMGHE: Historical Report 1948.

222 Ebenda.

223 OMGHE: Historical Report July 1945.

224 OMGHE: Historical Report October 1945 - June 1946. 
die Übergabe von Reichs- und Ländereigentum an deutsche Stellen. Wiedergutmachungsmaßnahmen waren von deutschen Behörden unter Aufsicht von Property Control Division durchzuführen.

Nach der Bildung des Landes Hessen im Oktober 1945 übergab OMGHE schrittweise die Vermögenskontrolle an deutsche Behörden. Die Property Control Division kontrollierte deren Arbeit über das Landesamt für Vermögenskontrolle beim hessischen Finanzministerium. Eine Klärung der Verhältnisse erbrachte die OMGUS Directive "Übertragung der Verantwortung der Vermögenskontrolle in Groß-Hessen und Württemberg-Baden auf die Landesministerpräsidenten“ vom Mai 1946. Diese Directive übertrug die Gesamtverantwortung für die Vermögenskontrolle auf das Landesamt für Vermögenskontrolle und Wiedergutmachung (Land Civilian Agency Head) im Finanzministerium und seine nachgeordneten Behörden. Die Property Control Division war zwar weiterhin Kontrollorgan auf allen Ebenen, blieb aber nur für Vermögen von Angehörigen von Staaten der Vereinten Nationen und neutraler Staaten direkt zuständig. Mit Inkrafttreten dieser Regelungen zum 1.Juni 1946 erloschen die Property Control Aufgaben der Kreis- und Regierungsbezirksdetachments. Bis dahin waren in Hessen bereits 21561 Vermögenswerte unter Kontrolle gestellt worden. 225 Diese Zahl stieg bis auf 47010 am 1. Januar 1948 . 1948 kam es dann verstärkt zu Rückgaben und Veränderungen der Eigentumsverhältnisse, so daß Ende des Jahres nur noch 20360 Vermögenswerte unter Kontrolle standen. ${ }^{26}$ Das Landesamt für Vermögenskontrolle hatte die Tätigkeit der Militärregierungsstellen zunächst mit insgesamt 824 Beschäftigten fortgesetzt. ${ }^{227}$ Diese Zahl verringerte sich auf 614 am 1.August $1948 .^{28}$

Was die Rückgabe von durch NS-Organisationen beschlagnahmtem Eigentum be-

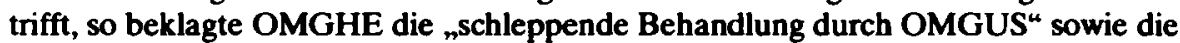
„Verwirrung und Ineffektivităt der deutschen Ämter für Vermögenskontrolle“. Dies hätte zu einer Verzögerung der Rückgabe um einen Zeitraum von bis zu drei Jahren geführt und beispielsweise bei den Gewerkschaften „beträchtliche Kritik [...], die weitgehend berechtigt war", hervorgerufen. Bei ausländischem Eigentum unterstellte OMGHE den deutschen Stellen sogar „absichtliches Verzögern“ und „Behinderung“ der Rückgabe. ${ }^{229}$

Die Spannungen zwischen OMGHE und OMGUS spitzten sich weiter zu, nachdem die OMGUS Property Control Branch 1948 eine AuBenstelle in Wiesbaden errichtet hatte. Nur durch die vom Chief of Property Control Division persönlich vorgetragene Drohung, daB OMGHE die Verantwortung fur das hessische Landesprogramm zur Vermögenskontrolle zurückgebe, sei es gelungen, OMGUS von „Einmischungen“ abzuhalten.

Nachdem OMGHE schon 1946 die Aufsicht uber deutsche Vermögen an das Land Hessen abgetreten hatte, erhielten die hessischen Behörden am 1. April 1948 auch die Zuständigkeit für ausländische Vermögen. Die Property Control Division konnte sich damit auf die Kontrolle der deutschen Maßnabmen beschränken. ${ }^{230}$

Im letzten Jahr der Militärregierung hatte die Entflechtung in der Arbeit der am 20. April 1948 gebildeten Property Division große Bedeutung. Am 12. Februar 1947

\footnotetext{
225 Ebenda.

226 OMGHE: Historical Report 1948.

227 OMGHE: Historical Report April - July 1947.

228 OMGHE: Historical Report 1948.

229 Ebenda.

230 Ebenda.
} 
hatten die Militärgouverneure der amerikanischen und der britischen Zone Entflechtungsgesetze verkündet (US-Militärregierungsgesetz Nr.56 / Britische Militärregierungsverordnung Nr.78 - Verbot der übermäBigen Konzentration deutscher Wirtschaftskraft). Danach wurde auf bizonaler Ebene die Bipartite Decartelization Commission (BIDEC) gebildet, die ihren Sitz in Frankfurt hatte. BIDEC richtete bei allen Ländermilitärregierungen Branch Offices ein. OMGHE siedelte ihre Decartelization Branch zunächst in der Economics Division an. Nach Auflösung der Economics Division im April 1948 setzte die Branch ihre Arbeit in der Property Division fort.

Bei der Entflechtungspolitik ging OMGHE von folgenden Überlegungen aus: „Eine der kostbarsten Wirtschaftstraditionen der Vereinigten Staaten besteht darin, Zusammenschlüsse von Industrieunternehmen dann zu zerschlagen, wenn ihre Bedeutung und ihr EinfluB so groß werden, daB sie faktisch den Markt monopolisieren und eine Gefahr für den freien Wettbewerb darstellen. Der Aufstieg von Industriegiganten war immer und unvermeidlich mit einer riesenhaften Zusammenballung politischer Macht verbunden." Mit dieser optimistischen Sichtweise der amerikanischen Wirtschaftstradition war die Einschätzung der deutschen Entwicklung verbunden, die Fragmente einer kritischen Faschismusanalyse enthielt: „Die Vereinigten Staaten hatten festgestellt, daB die Nazi-Partei ihre ersten finanziellen Stimuli von den riesigen zentralisierten Industrieunternehmen in Deutschland erhalten hatte. Sie waren sich weiter darüber im klaren, daß das Bündnis zwischen Big Business und der politischen Staatsführung zu einer hierarchischen Regierungsstruktur geführt hatte, in der das Führerprinzip angelegt war. Deshalb brachten die Vereinigten Staaten bei der Besetzung ihre feste Entschlossenheit - aus ihrer eigenen langen Tradition entsprungen - nach Deutschland mit, Monopole, Unternehmensverflechtungen und Kartelle aufzubrechen, damit sie nie wieder zu einer neuen Diktatur in Deutschland beitragen können." Ziel war, „für das deutsche Volk eine freie Wettbewerbswirtschaft wiederherzustellen, oder anders ausgedruckt, dem deutschen Volk seine wirtschaftliche Freiheit zurückzugeben. Dabei war völlig klar, daß politische Freiheit ohne wirtschaftliche Freiheit unmöglich ist". 2.31

Die praktische Entflechtungspolitik blieb jedoch deutlich hinter diesen wohlklingenden Willenserklärungen zurück. So heißt es zum Beispiel in einem internen Memorandum des Bipartite Control Office, US Decartelization Element (Frankfurt) vom 19. Juli 1949, daß das Entflechtungsprogramm auf Länderebene „nicht so gründlich und wirkungsvoll umgesetzt worden ist, wie es wünschenswert wäre". Etliche Militärregierungsoffiziere und deutsche Beamte hätten die Entflechtung für eine Sache gehalten, die „nur auf dem Papier stehe, für die praktische Politik aber bedeutungslos sei“. Viele deutsche und amerikanische Beamte hätten in der Entflechtung „ein destruktives Programm zur ,Atomisierung der deutschen Industrie“" gesehen. Sie hätten „passiven Widerstand“ gegen die Entflechtung geleistet, der zu „mangelhaften Fortschritten" im Entflechtungsprogramm geführt habe. Die Koordination sei unzureichend, die Decartelization Branches der Länderregierungen verfügten über zu wenig EinfluB und zu wenig Personal. Die anderen Abteilungen der Militärregierungen lieBen die Decartelization Branches ,im Regen stehen“. Dies fände seinen Ausdruck auch darin, daß die Chiefs of Branch in Gehalt und Rang niedriger eingestuft seien als ihre Kollegen. Die rote Laterne hätte in dieser Hinsicht der Chief of Branch in der hessischen Militärregierung. ${ }^{232}$ Mit dieser kritischen Analyse und seinen Verbesse-

231 Ebenda.

$23217 / 8285 / 19$. 
rungsvorschlägen stand das Memorandum jedoch auf verlorenem Posten. Bereits im April 1949 hatte Lucius D. Clay erklärt, daB jetzt „der Zeitpunkt gekommen“ sei, ,in dem die Entkartellisierung beendet werden muB“. ${ }^{233}$ Ein Memorandum des Leiters der hessischen Decartelization Branch vom 11. Juli 1949 beklagte, daß aufgrund der Bewirtschaftungsmaßnahmen in verschiedenen Bereichen „das Entflechtungsprogramm stark behindert" worden sei. ${ }^{234}$

Hatten sich „die ersten Aktivitäten der OMGHE Decartelization Branch darauf beschränkt, die deutsche Industrie ausführlich zu studieren und zu untersuchen“, so konnte im Jahr 1948 die Untersuchung von 452 hessischen Betrieben abgeschlossen werden. 264 Abschlußberichte mit Entflechtungsvereinbarungen wurden an OMGUS zur Überprüfung weitergeleitet. Im gleichen Jahr wurden 82 sogenannte „Show Cause Orders“ vorbereitet und BIDEC zur Unterzeichnung vorgelegt. Mit diesen „Show Cause Orders" wurden Firmen oder Einzelpersonen gezwungen, schriftlich zu begründen, warum - ihrer Meinung nach - eine von ihnen geplante oder durchgeführte wirtschaftliche Aktivität dem Militärregierungsgesetz Nr. 56 nicht widerspräche. ${ }^{235}$

„Beträchtliche Fortschritte“ seien 1948 auch „,in der gewaltigen Aufgabe, die IG-Farben-Gruppe zu entflechten, gemacht worden“. ${ }^{236}$ Der Konzern war zunächst unter Aufsicht des Control Office, G-5, USFET, gestellt worden. Am 8. März 1946 erhielt die Property Control Division, OMGHE, die Zuständigkeit für den Frankfurter Konzern zugesprochen und begann am 15. März mit den notwendigen Erfassungsmaßnahmen. 237

Erst 1948 jedoch wurden die in Hessen gelegenen Teile der IG-Farben in siebzehn unabhängige Unternehmen umgewandelt. Vorschläge für die weitere Entflechtung des IG-Farben-Vermögens in der Bizone sollte ein aus Deutschen bestehendes „Farben Dispersal Panel“ (FARDIP) erarbeiten. Die Produktion der IG-Farben konnte während der gesamten Entflechtungsperiode weiterlaufen. ${ }^{238}$

Ausgehend von der fur die Entflechtung formulierten Zielsetzung beschäftigte sich die Division auch mit den berufsständischen Zulassungsverfahren. Sie wies zahlreiche Gesetzesvorlagen zurück und verlangte, daß der freie Wettbewerb auch bei der Berufsausübung in keiner Weise eingeschränkt werden dürfe. ${ }^{239}$

Wie die Decartelization Branch hatte auch die Monuments, Fine Arts and Archives Section (MFAA) bis zum April 1948 zur Economics Division gehört und kam erst danach zur Property Division. Sie hatte zunehmend zwischen deutschen Kultureinrichtungen und ausländischen Staaten, die verschlepptes Kulturgut zurückforderten, zu vermitteln. Das Offenbach Archival Depot konnte seine Bestände bis Ende Juni 1948 um rund eine Viertelmillion Bücher reduzieren, nachdem es deren rechtmäßige Besitzer ausgemacht hatte.

Trotz einiger Reibereien machte auch die Rückführung von Kunstschätzen und Museumsobjekten aus dem Wiesbaden Control Collecting Point Fortschritte. In einer spektakulären Aktion wurden im Sommer/Herbst 1948106 weltbekannte Bilder, die früher dem Kaiser-Friedrich-Museum in Berlin gehört hatten, aus den Vereinigten Staaten nach Wiesbaden gebracht und ab 16. Oktober 1948 der Öffentlichkeit in einer

\footnotetext{
233 Wiesbadener Kurier vom 29.4.1949 (Ausschnitt in 17/7285/19).

$23417 / 8285 / 19$.

235 OMGHE: Historical Report 1948.

236 Ebenda.

237 OMGHE: Historical Report October 1945 - June 1946.

238 OMGHE: Historical Report 1948.

239 Ebenda.
} 
Ausstellung präsentiert. Die Bilder waren zusammen mit 96 anderen 1945 in die USA geschafft worden. In eine schwierige Situation geriet die Monuments, Fine Arts and Archives Section, als OMGUS 1948 alle hessischen Gesetze, die den Kunsthandel reglementierten, aufhob. MFAA hatte die Gesetze gutgeheißen und befürchtete jetzt die Rückkehr von Geschäftemachem und Nationalsozialisten auf dem Kunstmarkt.

\section{b) Organisation}

Die Property Control Division war zunächst in drei Branches gegliedert:

- Research and Records arbeitete Pläne aus, um Vermögen aufzuspüren, das in den Zuständigkeitsbereich von Property Control fiel. Die Branch wertete Erhebungsdaten aus - auch von den Geheimdiensten - und gab der angehängten Investigations Section Anweisungen für eigene Nachforschungen. Mit Hilfe von dadurch erhaltenen Informationen versuchte sie, unrechtmäBige Verschiebungen von beschlagnahmtem Vermögen aufzudecken und zu verhindern. Die Branch bearbeitete auch die Anträge auf Freigabe ausländischen Eigentums.

Die angehängte Legal Section (später Legal Counsel) überprüfte die Übereinstimmung der deutschen Vermögenskontrolle mit den Vorschriften der Militärregierung. Sie kontrollierte weiter die mit den Treuhändern geschlossenen Verträge auf ihre Rechtmäßigkeit. Später beschäftigte sie sich mit den hessischen Gesetzentwürfen, die Vermögenskontrolle und Wettbewerbsrecht betrafen.

- Accounting Control war für die praktische Durchführung der Property Control Politik zuständig, d. h. sie schloB Verträge ab, regelte die finanziellen Angelegenheiten usw. Die Branch war auch für die innere Verwaltung der Division zuständig. Ihre Accounts and Audits Section überprufte die Berichte der Treuhänder, während Statistical Section das Zahlenmaterial zusammenstellte.

- In Field Supervision waren die sieben Außendienstmitarbeiter (Field Supervisory Representatives) zusammengefaßt, die die Arbeit der deutschen Vermögenskontrollbehörden überwachten und bis 1948 für die Verwaltung des ausländischen Vermögens zuständig waren.

Damit hatte die Property Control Division zunächst folgende Struktur: ${ }^{240}$

Ubersicht 54: Organisation der Property Control Division, OMGHE, Juni 1946

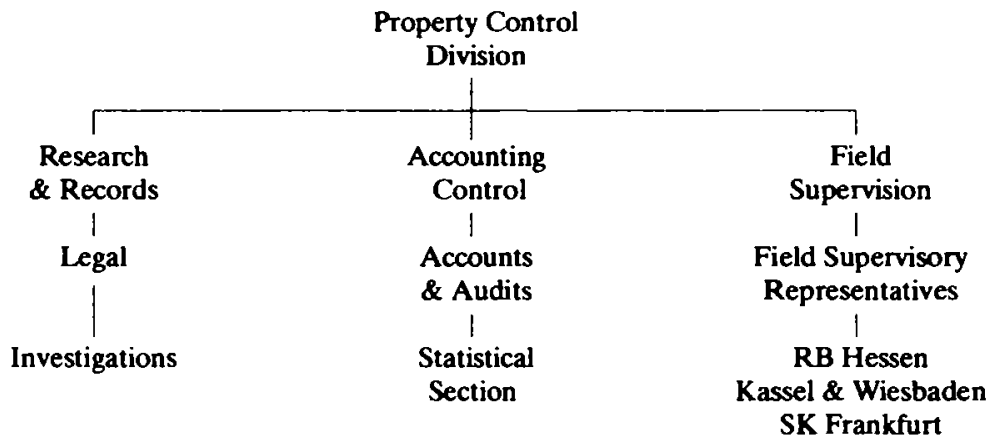

${ }^{240}$ OMGHE: Historical Report October 1945 - June 1946. 
Bis Frühsommer 1947 wurden die koordinierenden und administrativen Aufgaben in der Executive Branch zusammengefaßt, an die Legal Counsel als jetzt eigenständige Section angehängt wurde. Entsprechend der weiteren Übertragung praktischer Vermögenskontrolle auf deutschen Stelle wurde die Field Supervision in German Agencies Branch umbenannt. Selbst abzuwickeln blieb für die Property Control Division nur mehr die ausländisches Eigentum betreffenden Angelegenheiten. Die Investigations Section kam jetzt von Research zur German Agencies Branch.

Damit hatte die Division folgendes Aussehen: ${ }^{241}$

Ubersicht 55: Organisation der Property Control Division, OMGHE, 30. Juni 1947

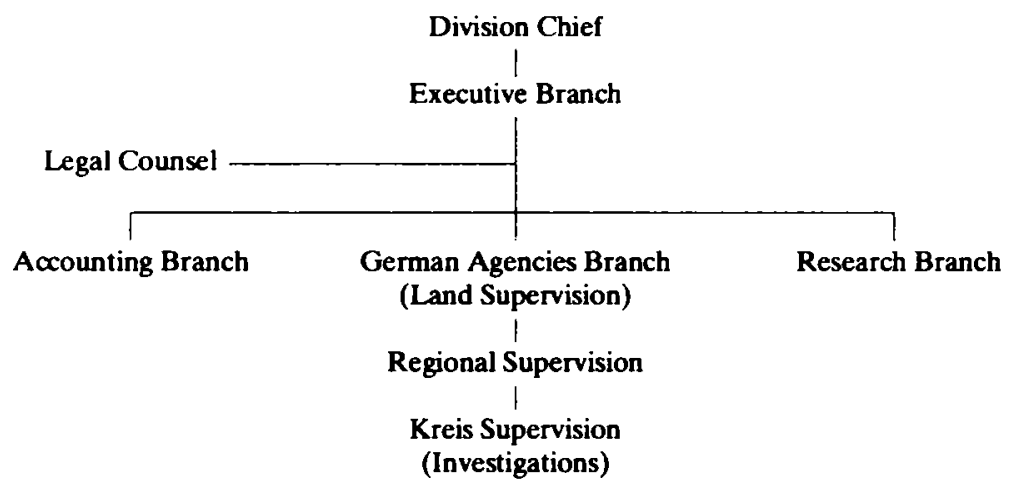

Veränderte Aufgaben bedingten eine Umorganisation der Division zum Jahresende 1947. Über die unter Kontrolle genommenen Vermögen mußte jetzt endgültig entschieden werden: Sollten sie an die früheren Eigentümer zurück- oder an neue weitergegeben werden? Internal Restitution bearbeitete jetzt die unter Militärregierungsgesetz Nr.59 (Rückerstattung feststellbarer Vermögensgegenstände) vom 10. November 1947 fallenden NS-Vermögen, wăhrend Disposition-Decontrol für alle Vermögen der im Kontrollratsgesetz Nr. 2 aufgefuhrten Organisationen und für die mit OMGUS-Direktive vom 25. Juni 1947 benannten ausländischen Vermögen zuständig war. Legal Counsel wurde an Internal Restitution angehängt; mit der inneren Verwaltung der Division war jetzt eine dem Deputy Director verantwortliche Section beauftragt.

Die Division hatte damit bis zur Umstrukturierung am 20. April 1948 die in Übersicht 56 wiedergegebene Organisation. 242

General Order Nr.5 vom 20. April 1948 bestimmte die Auflösung der Property Control Division und die Bildung der Property Division. In den folgenden Monaten gliederte sich die neue Division in vier Branches und zwei Sections. Eine dem Deputy Director verantwortliche Section war für die Verwaltung zuständig. Property Control and Internal Restitution Branch war für alle deutschen Vermögen betreffenden Aufgaben der früheren Property Control Division sowie für die Kontrolle der deutschen Ämter für Vermögenskontrolle zuständig. Von der früheren Economics Division kam die Decartelization Branch mit ihren zwei Außenstellen in Frankfurt und Kassel zu der neuen Division. Die Reparations Branch war gleichfalls vorher bei der Economics Division gewesen. Ihr fielen auch die Aufgaben der früheren Restitution Section der 
Ubersicht 56: Organisation der Property Control Division, OMGHE, 31. Dezember 1947

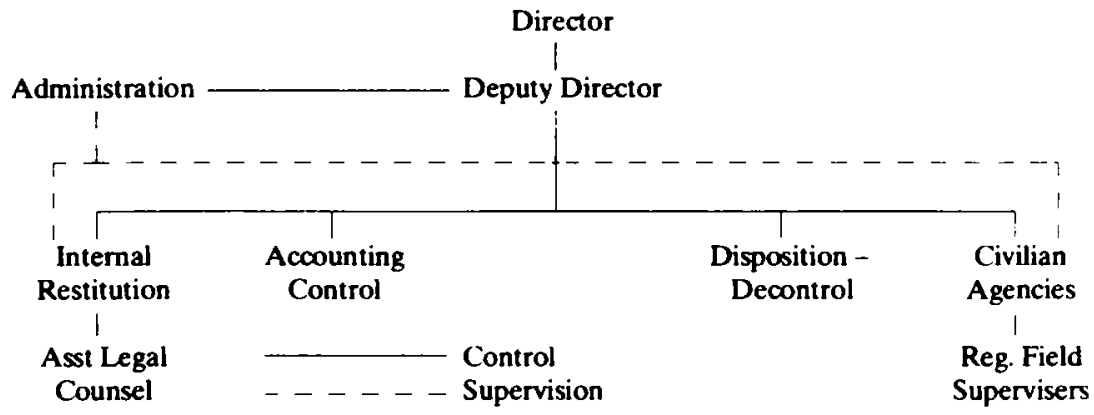

Economics Division zu. ${ }^{243}$ Für ausländisches und im Ausland befindliches Vermögen war die External Restitution Branch zuständig. An sie war die Monuments, Fine Arts and Archives Section - ebenfalls früher bei der Economics Division - angehängt. Bis Juli 1949 hatte die Property Division damit folgende Organisation:244

Ubersicht 57: Organisation der Property Division, OMGHE, 30. Juni 1948

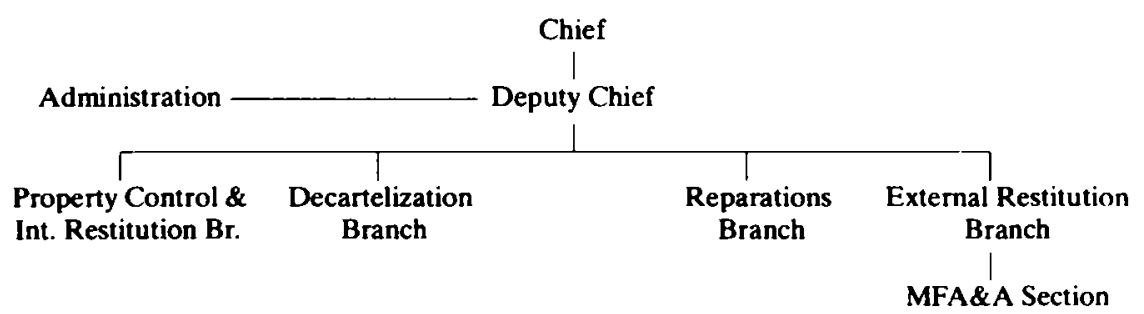

Da sich die Aufgaben der Divison immer weiter verringerten, wurde sie im Juli 1949 aufgelöst. Die verbleibenden Arbeiten führte die Property Branch der neu zusammengestellten Economics Affairs Division aus. Nur die Decartelization Branch blieb als eigenständige Einheit in der neuen Division erhalten.

\section{c) Personal}

Die Property Control Division zählte nach ihrer Größe zu den mittleren Divisions. Sie hatte 1946 14, am 1.Januar 1947 13, am 30. Oktober 1947 zwölf, am 1.Januar 1948 neun und am 15. März 1948 zehn amerikanische Mitarbeiter. Nach der Neustrukturierung im April 1948 stieg deren Zahl vorübergehend auf 17 an, um zum Jahresende wieder auf zehn zu sinken. Im Mai 1949 arbeiteten dann nur noch vier Amerikaner in der Division.

Die Zahl der deutschen Mitarbeiter betrug 1946 17. Am 1.Januar 1947 waren 20, am 30. Oktober 194718 und von Januar bis März 1948 wieder 17 Deutsche bei Property Control beschäftigt. Nach der Neustrukturierung erreichte die Zahl der Deutschen vorübergehend mehr als 20.

${ }^{243} \mathrm{Zu}$ den Aufgaben von Reparations und Restitution vgl. OMGUS-Hb, Teil Hessen B.5.

$2445 / 41-3 / 10$. 
Division Chief war vom Juli bis September 1945 Major Myron M. Maupin. September/Oktober 1945 hatte Cap. Francis R. Patemoster dieses Amt inne. Maupin und Paternoster waren schon bei der Aufstellung von E1 A2 am 15. September 1944 dabei gewesen. Von Oktober 1945 bis Januar 1946 leitete Lt.Col. William K. Denaro die Division. Ihn löste Lt.Col. Krit G. Logsdon ab, der gleichzeitig Chief der Legal Division war. Auf Logsdon folgte schon im März 1946 Major Irwin A. Urbantke, der bis Oktober tätig war. Eine längere Amtszeit hatte William R. Rule. Er wurde erst im Juni 1949 von John R. Cain abgelöst, der seit Ende 1947 sein Stellvertreter gewesen war. Vor diesem Zeitpunkt hatte die Division keinen Deputy Chief gehabt.

Die größte Branch der Property Control Division war zunächst Field Supervision/German Agencies, die 1946 neun Mitarbeiter umfaBte, darunter sieben Field Representatives. Angesichts der Hinwendung zur De-Control schrumpfte diese Branch bis auf einen Mitarbeiter im Dezember 1948 zusammen. Chief of Branch war lange Zeit Victor G. Cronthall gewesen. Er übernahm Anfang 1949 die Leitung der Decartelization Branch von Erich L. Meyer, der diese Branch nach der Neustrukturierung im April 1948 geleitet hatte. Cronthall war nach der Ernennung von J. R. Cain zum Chief of Division gleichzeitig auch Leiter der Property Control Branch, die zuvor Cain geleitet hatte. Chief der Reparations Branch in der Property Division war Emest J. Bootz, der zuvor in der Economics Division tätig gewesen war. Ebenso führte Theodore A. Heinrich in der Property Division sein Amt als Monuments, Fine Arts and Archives Chief fort, das er bereits in der Economics Division innegehabt hatte. Chef der kleinen Accounting Branch (später External Restitution) war Allan C. Wilson.

\section{Public Health Division}

\section{a) Aufgaben}

„Entnazifizierung und Wiederaufbau des öffentlichen Gesundheitswesens sowie die Seuchenkontrolle" waren zunächst Hauptaufgaben der Public Health Division. ${ }^{245}$ Dazu kamen die Überwachung der Ernährungssituation, des Krankenpflegewesens und der Tiermedizin. Für die Versorgung mit Arzneien und medizinischem Gerät war die Division gemeinsam mit der Economics Division zuständig.

Die Public Health Aufgaben hatten anfangs die Public Health Sections der Kreisdetachments wahrgenommen. Diese bestanden aus einem Offizier und einer unterschiedlichen Anzahl von Soldaten. Die Public Health Sections wurden im Dezember 1945 aufgelöst, da zu diesem Zeitpunkt die Abteilung für Gesundheitswesen des am 12. Oktober 1945 mit Organizational Direction Nr. 2, OMGHE, gebildeten hessischen Innenministeriums und seine nachgeordneten Stellen die praktischen Arbeiten übernahmen. Die Abteilung für Gesundheitswesen wurde durch die Public Health Division (zunächst als Branch bezeichnet) von OMGHE kontrolliert.

Der Historical Report für die erste Phase der OMGHE-Tàtigkeit wertet diese Übergabe von Kompetenzen an deutsche Stellen als „verfrüht vom Standpunkt der Entnazifizierung“ aus betrachtet. Die Entnazifizierung sei für die Division zunächst das größte Problem gewesen. Es war offenbar schwierig, genügend unbelastete Ärzte und Amtsärzte für die medizinische Versorgung aufzutreiben. Besonders unangenehm war OMGHE die Mitwirkung der „politisch beeinflußten Ärztekammem“ bei der Zulas-

245 OMGHE: Historical Report October 1945 - June 1946. 
sung. Eine umfassende Kontrolle dieser Zulassung hätte zwar die „Entnazifizierung verbessert", sei aber angesichts des medizinischen Bedarfs nicht durchführbar gewesen. 246

$\mathrm{Da}$ in Hessen keine größeren Epidemien zu verzeichnen waren, wertete OMGHE die von ihr durchgeführte Seuchenkontrolle als ,sehr erfolgreich““ ${ }^{247}$ Der Arbeitsschwerpunkt der Division verlagerte sich 1946 von der Entnazifizierung und Seuchenkontrolle auf die Überwachung der Ernährungssituation. Von Juni bis November 1945 waren auf zonaler Ebene insgesamt sechs Nutrition Teams aufgestellt worden. Zwei davon operierten in Hessen, von denen eines aber im Februar 1946 wieder aufgelöst wurde. Nach ersten Kontrollen hatten diese Teams in Hessen acht zumeist bombengeschädigte Städte als neuralgische Punkte in der Lebensmittelversorgung herausgefunden. Diese Städte waren Frankfurt, Kassel, Darmstadt, Wiesbaden, Hanau, Gießen, Marburg und Bad Homburg. Ihnen galt die besondere Aufmerksamkeit der Public Health Division, die beispielsweise durch Kinderspeisungen Engpässe zu verhindern suchte. ${ }^{248}$ Als im September 1946 das amerikanische Personal im verbliebenen Nutrition Team auf einen Offizier verringert wurde, übernahmen zwei deutsche Teams in vollem Umfang die Aufgaben der früheren US-Teams.

Daneben ordnete die Militärregierung umfangreiche „Street-Weighing and SchoolWeighing Programs" (Passanten und Schüler wurden gewogen) an, um einen einigermaßen repräsentativen Überblick über die Ernährungssituation zu gewinnen. ${ }^{249}$ In der Seuchenbekämpfung stellten nur die Tbc-Fälle und die beängstigende Verbreitung von Geschlechtskrankheiten ein Problem dar. Mit steigender Einfuhr von Penicillin konnte jedoch auch hier Abhilfe geschaffen werden. 250

Im zweiten Vierteljahr 1947 mußten der Leiter und der stellvertretende Leiter der Abteilung für Gesundheitswesen im Innenministerium wegen anstehender Spruchkammerverfahren vom Dienst suspendiert werden. Die Situation im deutschen Gesundheitswesen stellte sich für OMGHE insgesamt als „kläglich“ dar. ${ }^{251}$ Trotz des schleppenden Aufbaus eines „effektiven und demokratischen deutschen Gesundheitssystems" gab die Militärregierung bis Ende des Jahres 1947 alle Aktivitäten in diesem Bereich nach und nach auf und beschränkte sich auf ihre Kontrollaufgaben. ${ }^{252}$

Erst im Früjahr 1948 konnte die Division eine Verbesserung der Ernährungssituation und der medizinischen Versorgung feststellen. Die Entnazifizierung des Gesundheitswesens stellte für die Militärregierung zu diesem Zeitpunkt kein Problem mehr dar. ${ }^{253}$

\section{b) Organisation}

Die Public Health Division legte erst Mitte 1947 ihren ersten Organisationsplan vor. Bis Ende 1946 bezeichnete sich der Leiter der Division weiter als Senior Public Health Officer und knüpfte damit an die militärische Organisationsstruktur vor Etablierung

246 Ebenda.

247 Ebenda.

248 Ebenda.

249 OMGHE: Historical Report July - December 1946.

250 OMGHE: Historical Report January - March 1947 und OMGHE: Historical Report April July 1947.

251 OMGHE: Historical Report July - September 1947.

252 OMGHE: Historical Report October - December 1947. Vgl. auch J. R. Newmans Memorandum vom $1.12 .1947(8 / 214-1 / 9)$.

${ }^{253}$ OMGHE: Historical Report 1948 
von OMGHE an. Er gehörte auch zu denjenigen Mitarbeitern der Militärregierung, die ihren militärischen Rang am längsten behielten.

Bis 1947 hatte sich dann folgende Organisation herausgebildet: 254

Übersicht 58: Organisation der Public Health Division, OMGHE, 30. Juni 1947

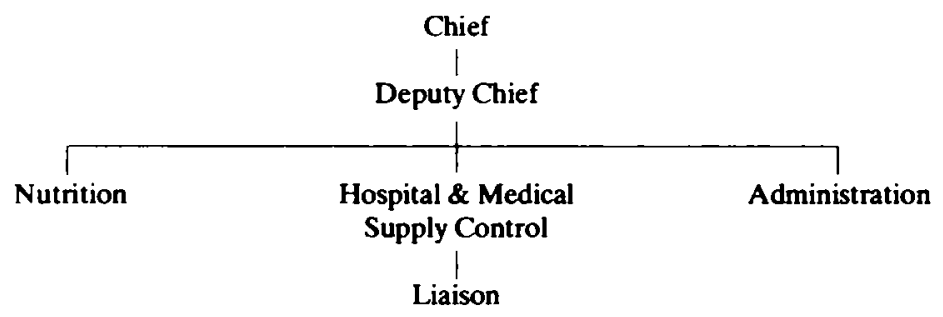

Der Chief kümmerte sich neben seinen üblichen Aufgaben um Ausbildungsangelegenheiten. Sein Stellvertreter war gleichzeitig für die Seuchenbekämpfung zuständig. Die Division war in drei Branches aufgeteilt. Die Nutrition Branch (Ernährung) kontrollierte die deutschen Nutrition Teams und sammelte Daten uber die Ernährungslage in Hessen. Die Hospital and Medical Supply Control Branch beschäftigte sich vor allem mit der Lagerung und Verteilung von Arzneimitteln (vorwiegend Penicillin) und medizinischem Gerät. Daneben kontrollierte und erfaBte sie die hessischen Krankenhäuser. An die Branch angeschlossen war ein Liaison Officer, der für die Verbindung mit den amerikanischen Truppen zuständig war. Die Administration Branch verwaltete die Division und unterstützte den Division Chief. Im Sommer 1947 wurde der Liaison Officer direkt beim Stab der Division und nicht mehr bei der Hospital and Medical Supply Control Branch angegliedert. In dieser Organisationsstruktur arbeitete die Division dann bis zu ihrer Auflösung am 20. April 1948. General Order Nr.5 vom 20. April 1948 löste die Division auf. Ihre Aufgaben wurden seit diesem Zeitpunkt von der Public Health Branch in der neu zusammengestellten Civil Administration Division wahrgenommen. Im Juli 1949 wurde die Branch mit der Public Welfare Branch derselben Division zur Social Affairs Branch zusammengefaßt.

\section{c) Personal}

Die Public Health Division wies einen sehr stabilen Personalstand auf. Von 1946 bis zu ihrer Auflösung beschäftigte sie fünf Amerikaner und sechs Deutsche. Nur die Zahl der Deutschen erhöhte sich im Herbst 1947 vorubergehend auf sieben. Nach der Auflösung der Division arbeiteten zunächst drei frühere amerikanische Mitarbeiter in der Public Health Branch der Civil Administration Division mit. 1949 blieb davon nur noch der Branch Chief übrig.

Vom Juli 1945 bis September 1946 hatten nacheinander sieben verschiedene Offiziere das Amt des Senior Public Health Officers inne. Dann übernahm Lt.Col. Charles H. Moseley diese Stelle (später als Division Chief). Er leitete die Division bis zu ihrer Auflösung. Danach wurde er Chief der Public Health Branch der Civil Administration Division und Public Health Adviser von J. R. Newman.

$2545 / 41-2 / 7$. 


\section{Public Information Division}

\section{a) Aufgaben}

„Military Government is news“. So beginnt ein Memorandum des Leiters der für die Öffentlichkeitsarbeit zuständigen Public Information Division vom 22. März 1948. ${ }^{255}$ Die kleinste Division von OMGHE schätzte die Bedeutung ihrer Arbeit also keineswegs gering. Dabei herrschte in den ersten Jahren der Militärregierung selbst bei der Benennung dieser Division ein Durcheinander, das weit über das übliche Maß hinausging. Sie begegnet uns zunächst als Public Relations Branch, dann als Public Relations Office oder als Public Information Section.

Erst in den letzten zwei Jahren von OMGHE setzte sich die Bezeichnung Public Information Division durch. Im ersten großen Historical Report beschrieb die Division/Branch ihre Aufgabenstellung so: „Seit ihrer Bildung im August 1945 ließ sich die Public Relations Branch von einem zentralen Motto leiten: die Militärregierung bei der Bevölkerung der USA und Hessens durch ein positives Bild in der Weltpresse gut zu verkaufen. “256

Die Aufgaben dieser Division sind ungefähr mit denen eines Presseamtes zu vergleichen: sie betreute Journalisten, gab Pressemitteilungen heraus, organisierte Pressekonferenzen und wertete für OMGHE Presse und Rundfunk aus. Etwas außergewöhnlich war die Arbeit der Home Town Branch, die die Medien in den Heimatorten der OMGHE-Mitarbeiter luber die Leistungen des Sohnes bzw. in Einzelfällen auch der Tochter der jeweiligen Stadt informierte.

Die Photographic Branch hatte neben der Arbeit für die Historical Reports - ein Supplementband war in der Regel mit Bildern und Pressemeldungen ausgestattet zusätzliche Service-Leistungen für Armeestellen und für OMGUS zu erbringen, wobei sie oft über ihre unzureichende Photo-Ausrüstung Klage führte. ${ }^{257}$

In der ersten Zeit war die Division einer strengen Kontrolle ausgesetzt. Sie mußte ihre Arbeit nicht nur mit 6871th DISCC abstimmen, ${ }^{258}$ sondern bis zum 1. Februar 1946 auch der 7.US-Armee ihre Pressemitteilungen vor der Herausgabe vorlegen. Nach Auflösung der 7. Armee lockerten sich diese Beschränkungen. Die Division arbeitete nun weitgehend selbstverantwortlich. Sie schickte Kopien ihrer Veröffentlichungen an die Public Relations Division, USFET, und konnte von einer quasi automatischen Freigabe ausgehen.

Pressemitteilungen, die die US-Zone betrafen, hatte die Division dem Public Relations Officer von OMGUS vorzulegen. Eine gewisse Kontrolle ergab sich zudem aus der Tatsache, daß die Public Information Division die meisten ihrer Mitteilungen über OMGUS oder USFET laufen ließ, da diese Stellen über bessere technische Verbreitungsmöglichkeiten verfügten. 259

Die inhaltlichen Arbeitsgrundlagen für die Division hatte der Assistant Executive Officer, OMGHE, in einem Memorandum vom 16. September 1946 zusammengefaßt. Er bezog sich dabei vor allem auf USFET Circular Nr. 152 vom 8. November 1945. Dieses Rundschreiben hielt die Public Relations-Stellen der Militärregierungen zu

255 „Militärregierung - das sind Nachrichten“ (8/215-1/2).

256 OMGHE: Historical Report October 1945 - June 1946.

257 OMGHE: Historical Report April - July 1947; OMGHE: Historical Report July - September 1947.

258 OMGHE: Historical Report November 1945.

${ }^{259}$ OMGHE: Historical Report October 1945 - June 1946. 
einem „liberalen und freundlichen Umgang“ mit Presseleuten an. Nach Aufhebung der Zensurmaßnahmen gegenüber Korrespondenten am 2. September 1945 sollten die Journalisten - sowohl Ausländer als auch zugelassene Deutsche - großzügig mit Material versorgt werden. Örtliche Beschränkungen seien aufzuheben. Ziel der amerikanischen Politik sei die allgemeine Pressefreiheit. Das Memorandum unterstrich abschließend, daß bei der hessischen Militärregierung für alle Fragen der Öffentlichkeitsarbeit die Public Relations Branch zuständig sei. ${ }^{260}$

In Absprache mit dem Direktor von OMGHE startete die Public Information Division hin und wieder auch gezielte Informationskampagnen. So trat sie beispielsweise nach einem Ersuchen der Decartelization Branch ${ }^{261}$ „unseriösen Stellen“ entgegen, die behauptet hatten, daß Entflechtung und Entnazifizierung dem wirtschaftlichen Wiederaufbau schadeten. ${ }^{262}$

\section{b) Organisation}

Schien schon bei etlichen anderen Divisions die graphische Darstellung ihrer inneren Organisation mehr von optischen und formalen als von tatsächlich existierenden arbeitspraktischen Gesichtspunkten geleitet, so wird dies bei der Public Information Division besonders deutlich. Als kleinste Division, die sie die meiste Zeit war, legte sie einen der differenziertesten Organisationspläne vor: ${ }^{263}$

Übersicht 59: Organisation der Public Information Division, OMGHE, 30. Juni 1947

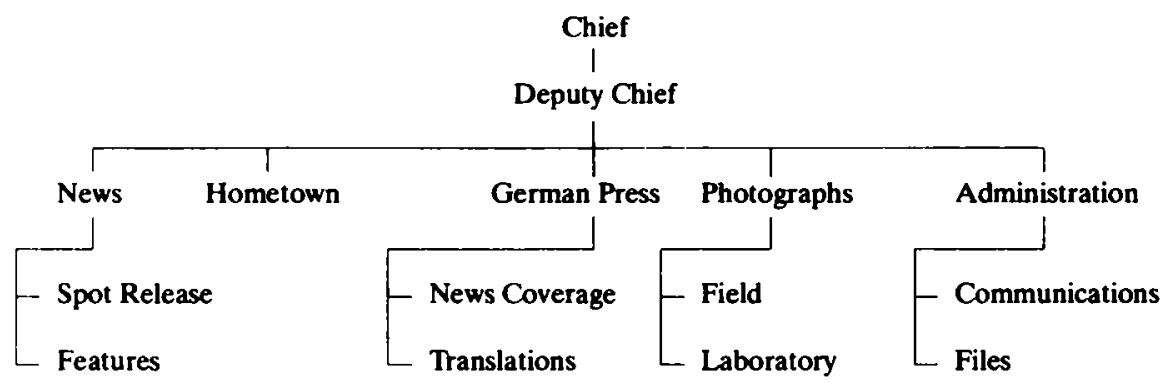

Angesichts der personellen Ausstattung können die darin aufgeführten einzelnen Branches und Sections nur als eine stichwortartige Wiedergabe der Aufgabenbeschreibung angesehen werden. Das hinderte die Division aber nicht daran, nach einer weiteren Personalkürzung im Jahre 1947 einen noch stärker ausdifferenzierten Organisationsplan vorzulegen (Übersicht 60 ). ${ }^{264}$

Im März 1948 kehrte die Public Information Division ohne erkennbaren Anlaß zum früheren Plan zurück, um im Juni - nach einer erneuten Personalkürzung - wieder den differenzierteren Plan vom Januar 1948 zu präsentieren.

\footnotetext{
$2608 / 217-1 / 3$.

261 17/8285/19.

262 OMGHE: Historical Report April - July 1947.

${ }^{263}$ Ebenda.

$2645 / 41-3 / 10$.
} 
Ubersicht 60: Organisation der Public Information Division, OMGHE, Januar 1948

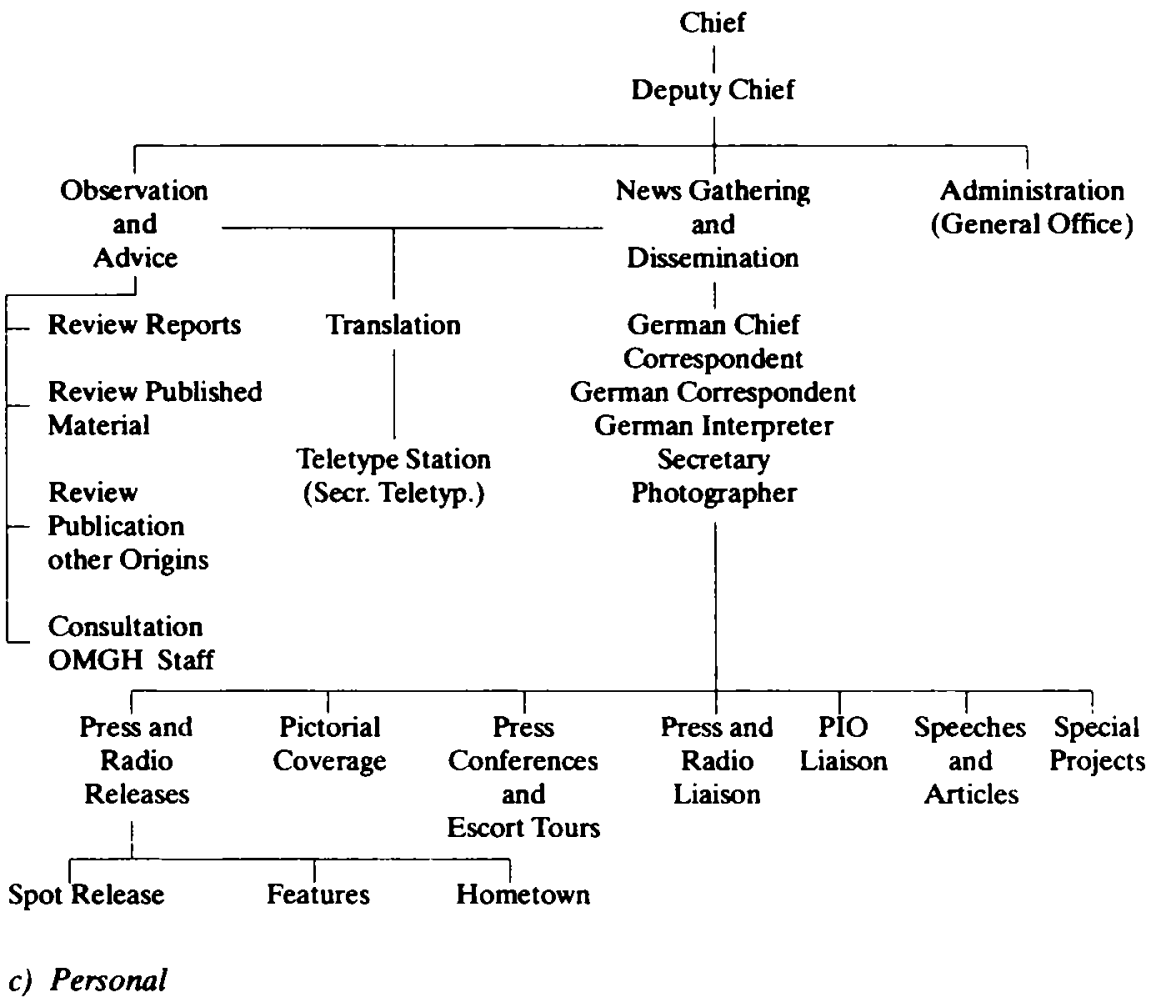

Im Jahre 1946 verfügte die Division über vier amerikanische Mitarbeiter. Bemerkenswert dabei ist, daß die Zivilbediensteten in dieser Division schon zu diesem Zeitpunkt wegen des hohen Anspruchs an die berufliche Qualifikation deutlich in der Mehrheit waren (drei). Anfang 1947 sank die Zahl der amerikanischen Mitarbeiter auf drei, Ende des Jahres auf zwei. Seit Mitte 1948 war dann nur noch ein Amerikaner, der Chief of Division, für die Public Information Division tätig. Demgegenüber stieg die Zahl der deutschen Beschäftigten von fünf im Jahr 1946 auf sieben Ende 1947 und blieb dann zumeist in dieser Höhe konstant.

In der Leitung zeichnete sich die Division durch Kontinuität aus. Hillard A. Rhoades leitete die Division von ihrer Bildung im Juli/August 1945 bis zum Ende von OMGHE.

\section{Public Safety Division}

\section{a) Aufgaben}

„In den ersten Monaten der Besetzung“, schrieb J. R. Newman rückblickend, „bezogen sich 85 Prozent der Militärregierungsarbeit auf den Bereich der öffentlichen Sicherheit““. ${ }^{265}$ Für diesen Bereich war seit Oktober 1945 die Public Safety Division

${ }^{265}$ Memorandum vom 1.12.1947 (8/214-1/9). 
(PSD) zuständig. „.Die Aufgabe, mit der sich die Public Safety Officers konfrontiert sahen, bestand darin, alle nazistischen Polizei- und Feuerwehrorganisationen aufzulösen und neue demokratische Polizei- und Feuerwehrorganisationen aufzubauen". heißt es im ersten Historical Report. ${ }^{266}$

Insgesamt äußerte sich OMGHE zufrieden über die öffentliche Sicherheit in Hessen: „Trotz ungünstiger Bedingungen - einem besiegten Volk, zerstörter Städte, Mangel an Lebensmitteln, Kleidung und Wohnraum, einer demoralisierten, unerfahrenen und unbewaffneten Polizei - war die Situation niemals alarmierend. "267 Auch die Aufhebung der Ausgangssperre am 30. März 1946 führte nicht zu einem Ansteigen der Kriminalität. ${ }^{268}$

Bis zum 22. Juli 1947 gehörte die für die Entnazifizierung zuständige Special Branch zur Public Safety Division. Mit über 700 deutschen und amerikanischen Mitarbeitern hatte diese Abteilung großes Gewicht in der Division. ${ }^{269}$ Die Public Safety Division war zunächst auch für das Gefängniswesen zuständig gewesen. Im Februar 1946 wurde ihre Prisons Branch jedoch der Legal Division unterstellt. ${ }^{270}$

Für Feuerwehr- und Polizeiangelegenheiten waren in der ersten Phase der Militärregierung die Public Safety Officers der örtlichen Detachments zuständig. Am 7. Juli 1945 hatte Section VII der USFET Directive „Administration of Military Government in the U.S. Zone of Germany" eine inhaltliche Grundlage für diese Arbeit geschaffen. Danach konnten kleine Einheiten von Stadt-, Land-, Grenz- und Kriminalpolizei aufgestellt werden. Bis September 1945 blieben diese Einheiten unbewaffnet. Seit diesem Zeitpunkt wurden sie nach und nach mit leichten amerikanischen Waffen ausgestattet. Bis zum 1.Juli 1946 waren in Hessen 4698 Stadtpolizisten in Dienst genommen. Die Militärregierung hatte sie zuvor auf ihre demokratische Gesinnung überpruft.

Die Organisation der Landpolizei wurde uber die Kreis- und Regierungsbezirksebene schrittweise zentralisiert. Eine USFET Directive vom 21.Januar 1946 erlaubte die Einrichtung einer Landespolizeidirektion, die in Hessen am 15. Mai 1946 in der Abteilung für offentliche Sicherheit im Innenministerium eingerichtet wurde. Mitte 1946 umfaßte die Landpolizei bereits 1300 Mann. Unter gleicher deutscher Verantwortlichkeit wurde zum 15. Februar 1946 eine Landesgrenzpolizei eingerichtet, die in den hessischen Grenzgebieten der US-Zone tätig war. Bis Sommer 1946 waren 636 Mann eingestellt, die in zwölf Kommissariate und 84 Posten aufgeteilt waren. Ein Landeskriminalamt war bereits zum 13. Dezember 1945 beim Innenministerium eingerichtet worden.

Der Aufbau der Feuerwehr verlief dezentral. Die Militärregierung achtete darauf, daB die Feuerwehren getrennt von der Polizei arbeiteten und jeden militärischen Drill aufgaben. Eine Kriegsfolge war die Einrichtung einer Spezialabteilung zum Entschärfen von Bomben, die der Brandschutzabteilung des Innenministeriums unterstand.

Ein weiterer Arbeitsschwerpunkt der Public Safety Division war die Ausgabe von Pässen und Passierscheinen. Nachdem im Juni 1946 mit den anderen Besatzungszonen Vereinbarungen über den gegenseitigen Reiseverkehr getroffen worden waren, nahm diese Aufgabe immer größeren Raum ein. Zunächst waren für diese Angelegenheiten die örtlichen Detachments zuständig gewesen. Nachdem im Januar 1946 in der Public Safety Division eine Pass Section eingerichtet worden war, übernahm das Wiesbade-

266 OMGHE: Historical Report October 1945 - June 1946.

267 Ebenda.

268 8/53-1/26.

${ }^{269}$ Zur Entnazifizierung vgl. OMGUS-Hb,Teil Hessen B.22.

${ }^{270}$ Chronology, S. 14; vgl. auch OMGUS-Hb, Teil Hessen B.13. 
ner OMGHE-Hauptquartier diese Aufgaben. Angesichts der zunehmenden Arbeitsbelastung stieg der Personalbestand der neuen Section in der ersten Jahreshälfte 1946 von drei auf 20 Personen. Täglich waren rund 1500 Fälle zu bearbeiten. ${ }^{271}$

Am 2. September 1947 erfolgte im OMGHE-Hauptquartier die Eröffnung eines Combined Travel Board, der die Verantwortung für die Ein- und Ausreise von bzw. nach Deutschland übernahm. Die fünf deutschen Mitarbeiter der Pass Section der Public Safety Division wechselten zu diesem Board. ${ }^{27}$ Während zum 20. August 1948 alle Handels- und Reisebeschränkungen mit der britischen und der französischen Zone aufgehoben worden waren, wurde der Interzonenverkehr mit der sowjetischen Besatzungszone - vor allem nach der Währungsreform - immer problematischer. ${ }^{273}$

In ihren Wochenberichten informierte die Public Safety Division über Probleme der öffentlichen Sicherheit wie z. B. Räuberbanden oder rechtsextremistische Aktivitäten. ${ }^{274}$ Wie aus einem Bericht des Public Safety Officer des Stadtkreises Wiesbaden vom Frühjahr 1947 hervorgeht, bestand eine Hauptaufgabe der Public Safety Division im Polizeibereich im Versuch, ein Bindeglied zwischen deutschen und amerikanischen Polizeistellen zu bilden. ${ }^{275}$ Schon zu Beginn der OMGHE-Tätigkeit hatten Public Safety Officers Vorträge für Mitarbeiter der US Constabulary gehalten. ${ }^{276}$

Trotz gelegentlicher Mißverständnisse ${ }^{277}$ und der Entfernung etlicher Belasteter aus dem Polizeidienst - nachdem die Akten des Berlin Document Center zugänglich waren - machte der Aufbau der deutschen Polizei in den Augen von OMGHE gute Fortschritte. ${ }^{278}$ Die deutsche Polizei nahm Anleitung und Hilfe von OMGHE entgegen und bezog sich bei ihren Maßnahmen zur Stärkung der eigenen Autorität oft auf die Militärregierung. 279

Das Polizeiwesen ging erst Anfang 1948 auf Grundlage einer OMGUS-Direktive vom 19. Januar 1948 endgültig in deutsche Verantwortung über. OMGHE äußerte sich insgesamt zufrieden über den Aufbau der deutschen Polizei in Hessen, beklagte aber das Weiterwirken „einiger rudimentärer Formen“" von Militarismus. ${ }^{280}$

\section{b) Organisation}

Die Public Safety Division war zunächst in fünf Unterabteilungen gegliedert:

- die Special Branch mit ihren Untersuchungs- und Inspektionsteams;

- die Police and Fire Branch mit Sections für die vier Polizeibereiche und für die Feuerwehr;

- die Pass Section für Reise- und Handelsangelegenheiten;

- die Field Investigations Branch zur Erstellung der Berichte zur Lage der öffentlichen Sicherheit.

Die Organisation der Division zeigte folgendes Bild: ${ }^{281}$

271 OMGHE: Historical Report October 1945 - June 1946.

272 OMGHE: Historical Report July - September 1947.

273 OMGHE: Historical Report April - July 1947; OMGHE: Historical Report 1948.

$2748 / 53-1 / 26$ und 8/188-2/6.

$2758 / 215-1 / 2$.

276 OMGHE: Historical Report October 1945 - June 1946.

27 OMGHE: Historical Report July - September 1947.

278 OMGHE: Historical Report April - July 1947.

279 OMGHE: Historical Report October - December 1947.

280 OMGHE: Historical Report 1948.

281 OMGHE: Historical Report October 1945 - June 1946, Annex. 
Ubersicht 61: Organisation der Public Safety Division, OMGHE, Juni 1946

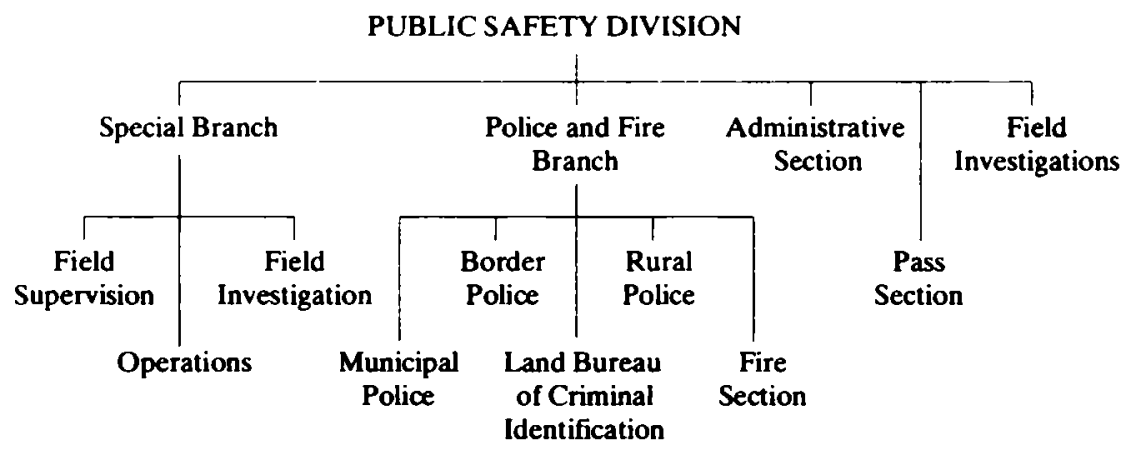

Im Sommer entfiel die Special Branch. Administrative und Pass Section wurden zusammengelegt und direkt beim Deputy Chief angebunden. Diese Section war auch für die Informationsverteilung zuständig. Die fruheren Sections der Police and Fire Branch wurden jetzt zu eigenen Unterabteilungen, von denen je eine fuir die

- Stadtpolizei

- Landpolizei und Grenzpolizei

- Feuerwehr und Bombenentschärfung

- Kriminalpolizei, Polizeifunk und Kriminalstatistik zuständig war.

Diese Struktur behielt die Division bis zu ihrer Auflösung am 20. April 1948:202

Ubersicht 62: Organisation der Public Safety Division, OMGHE, 31. Dezember 1947

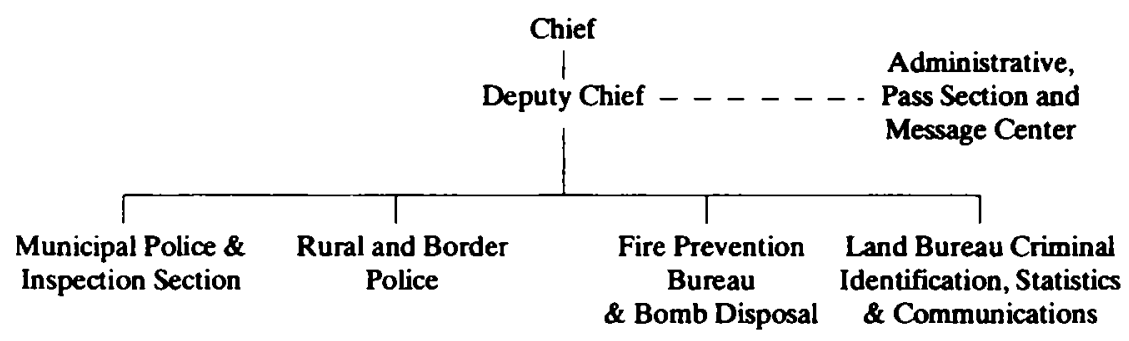

General Order Nr. 5 vom 20. April 1948 bestimmte die Auflösung der Public Safety Division. Ihre Aufgaben hatte jetzt die Public Safety Branch in der neu zusammengestellten Civil Administration Division wahrzunehmen. ${ }^{283}$ Der Branch Chief wurde mit General Order Nr.6 vom 22. April 1948 zum Public Safety Adviser des Direktors von OMGHE ernannt. ${ }^{284}$ 


\section{c) Personal}

Nach den ersten Monaten der Besatzungszeit gehörte die Public Safety Division zu den mittelgroßen Divisions. Ohne Special Branch hatte sie 1946 13, 1947 zwölf und vor der Auflösung im April 1948 zehn amerikanische Mitarbeiter. In der Public Safety Branch der Civil Administration Division waren zunächst sechs Amerikaner tätig. Bis zum Ende der Militärregierung verringerte sich das Personal bis auf eine Person, den Branch Chief.

1946 arbeiteten 23 Deutsche für die Public Safety Division (ohne Special Branch). Diese Zahl stieg Anfang 1947 vorübergehend auf 27 an, um dann kontinuierlich auf 11 bei der Auflösung der Division zu fallen.

Von der Bildung bis zum Januar 1946 wurde die Division von Curtis Reid geleitet. Ihn löste der spätere Executive Officer Ernest K. Neumann ab, der bis November 1946 tätig war. Vom November 1946 bis September 1948 leitete John E. Stuerwald, der vorher bei der Police and Fire Branch tätig gewesen war, die Division bzw. die Branch. Seit 22. April 1948 war er auch Public Safety Adviser des Direktors von OMGHE.

Nach Stuerwald leitete Hubert J. Teitelbaum vorübergehend die Branch. Teitelbaum war gleichzeitig Chief der Denazification Branch in der Civil Administration Division und kannte Public Safety von seiner früheren Tätigkeit als Chief der Special Branch. In den letzten Monaten vor Auflösung von OMGHE leitete dann der langjährige Mitarbeiter der Public Safety Division Jesse W. Callahan als letzter verbliebener Public Safety Experte die Branch.

\section{Public Welfare and Displaced Persons Division}

\section{a) Aufgaben}

Die Displaced Persons, definiert als „Zivilisten, die kriegsbedingt außerhalb der Grenzen ihrer Heimatländer leben“, 285 standen von Anfang an im Mittelpunkt der Militärregierungsarbeit. Schon während ihres Aufenthalts in Frankreich beschäftigten sich die später in Hessen tätigen Detachments intensiv mit diesem Problem. E1 A2 (E-5) hatte bereits zu diesem Zeitpunkt eine Displaced Persons Section gebildet. Ihre Mitarbeiter bereiteten sich unter anderem durch einen Aufenthalt bei der G-5 Abteilung der 3. US Armee vom 10. bis 14. Oktober 1944 in Nancy vor und informierten sich über die Einrichtung von DP-Camps und die technischen Möglichkeiten der Armee. ${ }^{286}$

In Hessen trafen die Detachments dann nach eigenen Schätzungen 200000 Displaced Persons an. Ungefähr 150000 gelangten bis Ende 1945 in ihre Heimatländer zurück. ${ }^{287}$ In den offiziellen Sammellagern waren bis Ende 1945 fast 60000 DPs registriert, darunter mehr als die Hälfte Polen (November 1945: 56978 DPs, 33049 Polen; Dezember 1945: 58264 DPs, 35633 Polen). Die Armee stellte zunächst zwei, später vier Transportkompanien zusammen, um die DPs in den von ihr betreuten rund dreißig Lagern in Hessen nach Nationalitäten zusammenzuführen und auf den Weg in ihre Heimatländer zu bringen. ${ }^{288}$ Über die Zuständigkeiten für die von der UNRRA (United Nations Relief and Rehabilitation Administration) verwalteten Lager bestanden zunächst offenbar Unklarhei-

285 OMGHE: Historical Report October 1945 - June 1946.

286 8/187-1/2.

287 OMGHE: Historical Report October 1945 - June 1946.

288 OMGHE: Historical Report November 1945; OMGHE: Historical Report December 1945. 
ten. Eine am 9.Oktober 1945 veröffentlichte USFET-Anordnung wies der Militärregierung die Aufsicht über die Lager zu. Mit Schreiben vom 10. Oktober 1945 wies deren Wiesbadener Zentrale darauf hin, daß die kommandierenden Offiziere der Kreisdetachments für das reibungslose Funktionieren der in ihrem Bereich gelegenen DP-Lager verantwortlich seien. ${ }^{289}$

Nach einer Anordnung des Hauptquartiers des 7. US Armee vom 14. Dezember 1945 übernahm dann jedoch die Armee zum 1.Januar 1946 von der Militärregierung die Verantwortung für die DP-Sammellager in Hessen. ${ }^{290}$ Die Militärregierung behielt die zuständigkeit für DPs, die sich außerhalb der Lager aufhielten. ${ }^{291}$ Die Lager selbst wurden weiter von UNRRA verwaltet. Das UNRRA-Personal, die alliierten Verbindungsoffiziere und alle anderen Mitarbeiter der Sammellager wurden nun der 7. US Armee zugeordnet. ${ }^{292}$ Nach der Auflösung der 7. Armee übernahm Ende März 1946 die 3. US Armee die Kontrolle über die DP-Lager. ${ }^{293}$ Am 15. März 1947 gingen die Lager dann in die Zuständigkeit der Military Posts über. ${ }^{294} \mathrm{Am} 21$. April 1947 erging ein Aufnahmestop in den Sammellagern. DPs, die außerhalb der Lager lebten, fielen jetzt unter deutsche Zuständigkeit. Nach Auflösung der UNRRA zum 1.Juli 1947 übernahm die PCIRO (Preparatory Commission for International Refugee Organization) die Lagerverwaltung. 295

Zum 31. Dezember 1948 waren noch 39910 DPs in hessischen Sammellagern registriert. Dieser „harte Kern“ von schwierig rückführbaren DPs stellte „ein Hauptproblem" der Militärregierung dar, das sie aber am Ende ihrer Tătigkeit in deutsche Hände übergab. ${ }^{296}$

Neben der Anfang 1946 auf Koordination und Verbindung reduzierten DP-Betreuung war die Public Welfare and Displaced Persons Division für folgende Aufgabenbereiche zuständig:

- Flüchtlinge und Vertriebene,

- Sozialfürsorge,

- Kinder- und Jugendwohlfahrt,

- private und staatliche Wohlfahrtsorganisationen,

- freiwillige Hilfsprogramme,

- Behinderte,

- Verfolgte des Naziregimes,

- Rückführung deutscher Kriegsgefangener. ${ }^{297}$

Von Mai bis Juli 1945 wurden diese Aufgaben direkt von den lokalen Detachments und den deutschen Kommunalverwaltungen wahrgenommen. Seit Juli übernahmen sowohl auf deutscher wie auf amerikanischer Seite die Institutionen auf Regierungsbezirksebene die Koordination der Aktivitäten im Sozialbereich. ${ }^{298}$

\footnotetext{
$2898 / 216-3 / 5$.

290 OMGHE: Historical Report October 1945 - June 1946, Appendix.

$2918 / 68-2 / 2$.

292 OMGHE: Historical Report October 1945 - June 1946, Appendix.

293 Chronology, S. 17.

$2948 / 190-2 / 11$.

295 OMGHE: Historical Report April - July 1947.

296 OMGHE: Historical Report 1948.

297 OMGHE: Historical Report October 1945 - June 1946; OMGHE: Historical Report October - December 1947.

298 OMGHE: Historical Report October 1945 - June 1946.
} 
Nach Einrichtung des hessischen Ministeriums für Arbeit und Wohlfahrt mit Organizational Direction Nr.2, OMGHE, vom 14. Oktober 1945 verlagerte OMGHE seine Tätigkeiten von direkten Eingriffen auf Überwachung und Kontrolle. Dazu wurde die Public Welfare and Displaced Persons Division gebildet. ${ }^{299}$

Die Militärregierung wertete schon im Juni 1946 die Entnazifizierung im Sozialbereich als abgeschlossen. Die NS-Wohlfahrtsorganisationen waren aufgelöst, und die konfessionellen und freien Wohlfahrtsorganisationen erhielten die Erlaubnis, ihre Arbeit wieder aufzunehmen. Im April/Mai 1946 liefen in Hessen amerikanische Hilfsprogramme an. Diese Hilfsprogramme wurden vom Council of Relief Agencies Licensed for Operation in Germany (CRALOG) koordiniert. CRALOG hatte acht Repräsentanten in der US-Zone. Die praktische Durchführung der Hilfsprogramme wurde von OMGUS an deutsche Wohlfahrtsorganisationen übergeben.

Einen Teil der CRALOG-Hilfe wandten die Verbände für Schulspeisungen auf, die vom „LandesausschuB Groß-Hessen für die Verteilung ausländischer Liebesgaben“ durchgeführt wurde. Das Komitee wählte für die Speisungen zunächst die Städte Darmstadt, GieBen, Offenbach, Kassel und Frankfurt aus, weil dort nach den Erhebungen der Public Health Division (OMGHE) der Hunger besonders groB war. ${ }^{300}$

Seit Ende 1945 verlagerte sich der Arbeitsschwerpunkt der Division. Flüchtlinge, Vertriebene, Kriegsteilnehmer wurden anstelle der DPs zu den brennendsten Problemfällen. Im Dezember 1945 rechnete die Public Welfare and Displaced Persons Division mit 500000 Menschen, die zusätzlich nach Hessen strömen würden. ${ }^{301}$

Gemeinsam mit der Manpower, Public Safety, Food and Agriculture (Economics), Public Health und der Finance Division bildete sie ein Planungskomitee, das seine Arbeit am 19. Juni 1946 aufnahm. ${ }^{302}$ Bis zum 30. Juni 1947 waren bereits über 700000 Menschen nach Hessen eingereist. Die hessische Bevölkerungszahl überschritt damit die Viermillionengrenze. ${ }^{303}$ Vor allem an den drei offiziellen Exchange Points strömten die Menschen ins Land: in Bebra aus der sowjetischen Zone, in Volkmarsen aus der britischen Zone und in Wiesbaden-Kastel (Mainz-Kastel) aus der französischen Zone. ${ }^{304}$ Bis zum 30 . Juni 1948 waren 841216 Menschen zusätzlich nach Hessen gekommen, die damit über zwanzig Prozent der Gesamtbevölkerung ausmachten. Die Militärregierung wandte sich gegen Bestrebungen von Stellen der Landesregierung, das Landesflüchtlingsamt aufzulösen. Sie beklagte das Desinteresse der lokalen Behörden und sorgte sich um die „laufend schlechter werdenden Beziehungen zwischen Flüchtlingen und einheimischer Bevölkerung “305 - ein Problem, das die Besatzungszeit allerdings überdauern sollte.

\section{b) Organisation}

Entsprechend ihrer vielfältigen Aufgabenstellung entwarf die Public Welfare and Displaced Persons Division zunächst einen sehr differenzierten Organisationsaufbau. Sie unterhielt Abteilungen für

\footnotetext{
$2998 / 68-2 / 2$.

300 OMGHE: Historical Report October 1945 - June 1946.

$3018 / 68-2 / 2$.

302 OMGHE: Historical Report October 1945 - June 1946.

303 OMGHE: Historical Report April - July 1947.

304 OMGHE: Historical Report October 1945 - June 1946.

305 OMGHE: Historical Report 1948.
} 
- Verwaltung,

- Ausbildungsfragen,

- Verfolgte des Naziregimes,

- Flüchtlinge, Vertriebene und Einwanderer (diese Abteilung war auch für die Überwachung der regionalen Militärregierungseinheiten zuständig),

- Behinderte,

- CRALOG-Aktivitäten,

- Jugendwohlfahrt,

- amerikanische Hilfe und

- Sozialfürsorge.

Die Division hatte damit im Juni 1946 folgende Struktur: $:^{306}$

Ubersicht 63: Organisation der Public Welfare and Displaced Persons Division, OMGHE, Juni 1946

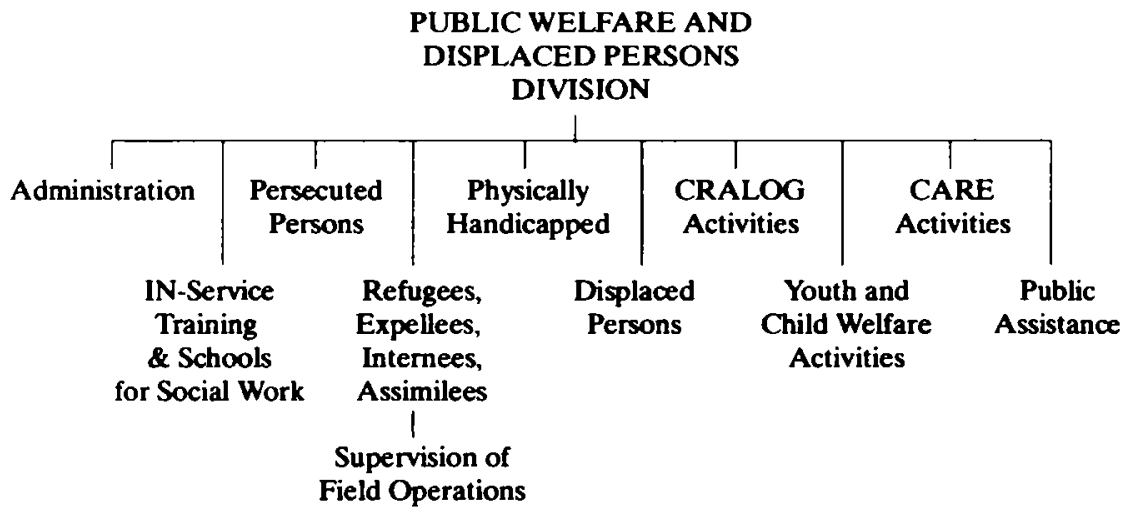

In den folgenden Monaten erfolgte eine Straffung des Organisationsplans, die dem realen Personalstand und Leistungsvermögen entsprach. Die Division hatte jetzt noch fünf Branches, die der Deputy Chief koordinierte:

- Displaced Persons Branch hielt in DP-Angelegenheiten Kontakt mit der Armee und mit deutschen Stellen. Sie war auch für Kriegsheimkehrer zuständig sowie für Fragen der Aus- und Einreise.

- Refugee and Expellee Resettlement Branch war für die Verteilung und Ansiedlung von Flüchtlingen, Vertriebenen und anderen Einwanderern zuständig.

- Field Representative überwachte die regionalen Aktivitäten.

- Public Assistance Branch war zuständig für Sozialhilfe, die Verbindung mit der hessischen Regierung, Fragen der hessischen Sozialgesetzgebung, Ernährungsprogramme, ausländische Hilfsorganisationen und Statistik.

- Welfare Services Branch beschäftigte sich mit Jugendwohlfahrt, Ausbildungsfragen, privaten Wohlfahrtsorganisationen, Verfolgten, Behinderten und Gemeindediensteten.

Die Division hatte damit im Juni 1947 folgende Struktur: 307

306 OMGHE: Historical Report October 1945 - June 1946, Annex.

307 5/41-27. 
Ubersicht 64: Organisation der Public Welfare and Displaced Persons Division, OMGHE, 30. Juni 1947

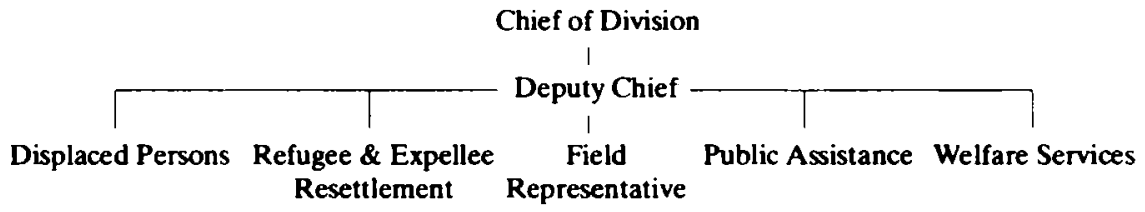

Bereits in den folgenden drei Monaten ergab sich eine weitere Straffung. Angesichts der geänderten Problemlage verlor die DP-Branch ihre Eigenständigkeit und wurde mit der Public Assistance Branch zusammengefaßt, die außerdem von der Welfare Services die Zuständigkeit für Verfolgte übernahm. Fragen der hessischen Landespolitik hatte sie jetzt nicht mehr zu regeln, dafür war der Chief of Division allein zuständig. An die Branch angegliedert war der CRALOG-Repräsentant in Hessen. Zwei Branches beschäftigten sich jetzt mit Flüchtlingsfragen. Auf zentraler Ebene arbeitete die Refugee and Resettlement Branch. Zu ihren bisherigen Aufgaben übernahm sie von der früheren DP-Branch die Zuständigkeit für Kriegsheimkehrer und Aus- und Einreiseangelegenheiten. Sie war zudem für die Überwachung der Exchange Points verantwortlich. Der Refugee and Expellee Control Officer und der Field Representative waren in einer weiteren Branch zusammengefaßt. Mit Ausnahme der Verfolgten behielt die Welfare Services Branch ihre bisherigen Zuständigkeiten. Diese Branch und die Refugee and Expellee Resettlement Branch wurden vom Chief of Division koordiniert, während der Deputy Chief für die anderen beiden Branches zuständig war. Die Division hatte damit seit September 1947 folgende Organisationsstruktur: ${ }^{308}$

Ubersicht 65: Organisation der Public Welfare and Displaced Persons Division, 30. September 1947

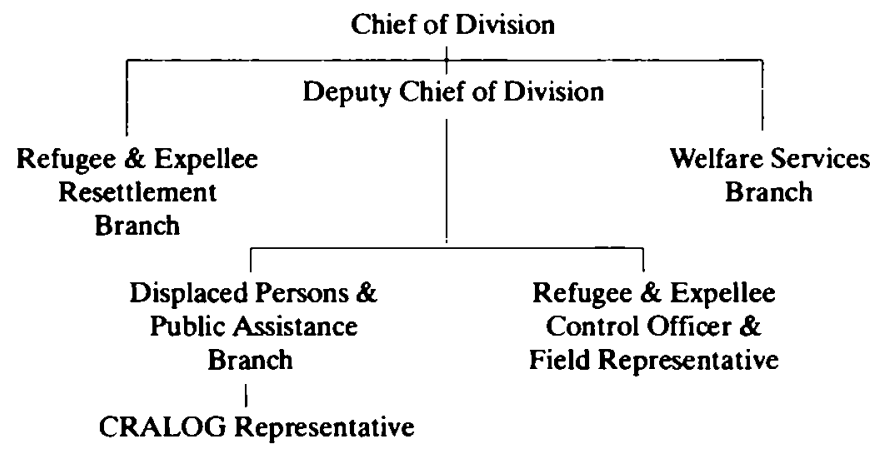

Diese Organisation behielt die Division bis zu ihrer Auflösung bei. General Order Nr. 5 vom 20. April 1948 löste die Public Welfare and Displaced Persons Division auf und übertrug ihre Aufgaben der Public Welfare and Displaced Persons Branch in der Civil Administration Division. Angesichts der abnehmenden Bedeutung der DP-Problematik hieB die Branch im letzten Jahr der Besatzungszeit nur noch Public Welfare

308 OMGHE: Historical Report July - September 1947. 
Branch. Der Chief of Branch wurde mit General Order Nr.6 vom 22. April 1948 zum Public Welfare Adviser des Direktors von OMGHE ernannt. Im Juli 1949 wurde die Branch mit der Public Health Branch zur Social Affairs Branch, Civil Administration Division, zusammengefaßt.

\section{c) Personal}

Angesichts der komplexen Aufgaben im Sozialbereich in einer wirtschaftlichen Notsituation nimmt sich die personelle Besetzung der Public Welfare and Displaced Persons Division sehr bescheiden aus. Die Division führte regelmäBig Klage wegen Überlastung und beantragte deshalb im März 1946 sogar, von der Abfassung eines wöchentlichen Arbeitsberichts befreit zu werden - allerdings ohne Erfolg. ${ }^{309}$

Die Zahl der amerikanischen Mitarbeiter wurde ganz im Gegenteil von acht im Jahr 1946 auf sechs im Jahr 1947 reduziert. 1948 sank die Zahl gar auf fünf. Nach Auflösung der Division arbeiteten noch vier Amerikaner in der Public Welfare Branch der Civil Administration Division. Am Ende der Militärregierungszeit blieb nur der Chief of Branch übrig. Was das deutsche Personal betrifft, so hatten die Klagen der Division mehr Erfolg. Die Zahl der deutschen Mitarbeiter stieg von vier 1946 auf zehn im Jahr 1947 und pendelte sich dann bei neun ein.

Bereits während der Tätigkeit von E-5 in Neustadt war Capt. Sharon L. Hatch Chief des Bereichs für Public Welfare and Displaced Persons gewesen. Er behielt diese Stelle bis Oktober 1945. Dann wurde Lt.Col. Charles H. Reed zum Chief of Division ernannt. Er wurde im Januar 1946 von Capt. Thomas A. Reardon abgelöst. Schon im März wurde Reardon aber zum Deputy Chief heruntergestuft; Chief wurde wieder Sharon L. Hatch, der dieses Amt bis zum SchluB innehatte. Er leitete auch die Public Welfare Branch in der Civil Administration Division und wurde am 22. April 1948 zum Public Welfare Adviser von J. R. Newman ernannt. Als Deputy Chief arbeitete nach Reardon Major Morris E. Sorenson und bis zum Wegfall der Stelle Peter Stanne.

\section{Special Branch / Denazification Division}

\section{a) Aufgaben}

Auf der Grundlage der USFET-Direktive vom 7. Juli $1945^{310}$ begannen die Special Branches der verschiedenen Detachments Entnazifizierungsverfahren durchzufuhren. ${ }^{311}$ Die Special Branches agierten zunächst im Rahmen der Public Safety Division. Betroffen waren Anwärter und Inhaber von Stellen im öffentlichen Dienst und privaten Sektor, deren Positionen von "mehr als geringer Bedeutung “ waren. ${ }^{312}$ Bis zum 31. Dezember 1945 waren in Hessen bereits 192558 Fragebogen ausgegeben worden. ${ }^{313}$

Die USFET-Direktive sah gleichzeitig die Einsetzung von Military Government Denazification Review Boards vor, die besonders wichtige Fälle von Amtsenthebungen

\footnotetext{
$3096 / 82-2 / 2$.

310 OMGUS: Report of the Military Governor (1. April 1947 - 30. April 1948), Nr.34, Denazification, S. 23 - 36; vgl. auch Niethammer: Mitläuferfabrik, S. 150 - 156.

311 8/214-1/11.

312 OMGUS: Report of the Military Governor (1. April 1947-30. April 1948), Nr.34, Denazification, S. 2; darin auch die weitere Entwicklung auf gesamtzonaler Ebene.

313 OMGHE: Historical Report December 1945.
} 
überprüften. ${ }^{314}$ Am 26. Dezember 1945 setzte OMGHE einen derartigen Board für das Land Hessen ein. Er bestand aus acht Offizieren der Militärregierung. Sekretär war 2 nd Lt. Roger E. Reynolds. Am 9. Februar 1946 ernannte der Direktor von OMGHE Arsen L. Yakoubian zum Vorsitzenden des Boards. Mit Befehlen vom 5. März und vom 22. April 1946 machte Newman zehn weitere Offiziere und amerikanische Zivilangestellte der Militärregierung zu Mitgliedern des Review Boards. ${ }^{315} \mathrm{Da}$ der Board mit drei anwesenden Mitgliedern beschluBfähig war, ${ }^{316}$ konnten aufgrund dieser Erweiterungen zahlreiche Fälle überprüft werden.

Bereits am 1.November 1945 wies ein Rundschreiben auf Probleme im Entnazifizierungsverfahren hin. Es verlangte mit großem Nachdruck, daB alle Sachbearbeiter die ausgefullten und bearbeiteten Fragebogen direkt zur Special Branch des Wiesbadener Militärregierungshauptquartiers weiterleiten und nicht, auch nicht als Kopien, bei sich behalten sollten. ${ }^{317}$

Eine neue Situation schuf das „Gesetz zur Befreiung von Nationalsozialismus und Militarismus", das nach Verkündung durch die Ministerpräsidenten von Bayern, Württemberg-Baden und Hessen mit Zustimmung der Militärregierung am 6. März 1946 in Kraft trat. ${ }^{318}$ Damit lag ab Juni 1946 die Verantwortung für die Durchführung des Entnazifizierungsprogramms in deutschen Händen. Nur noch in wenigen Bereichen, wie z. B. beim Personal des auf Grundlage von Artikel 23 dieses Gesetzes gebildeten Ministeriums für politische Befreiung oder von US-Einrichtungen, beteiligte sich die Militärregierung weiter am praktischen Entnazifizierungsverfahren. ${ }^{319}$

Die Arbeit der Special Branch konzentrierte sich jetzt auf die Überwachung des deutschen Vorgehens. OMGHE gliederte mit Anordnung vom 22. Juli 1946 die Special Branches aus der Public Safety Division aus und bildete eine eigenständige Special Branch Division, die Ende 1946 in Denazification Division umbenannt wurde. Durch die Bildung einer selbständigen Division sollte zudem eine direktere Kontrolle des für die Entnazifizierung zuständigen Ministeriums für politische Befreiung ermöglicht werden. ${ }^{320}$ Allerdings löste diese Umstrukturierung nicht alle Probleme: Am 13. September 1946 leitete Newman persönlich ein OMGUS-Schreiben vom 6. September an alle Liaison and Security Offices weiter, in dem die ,defätistische Haltung eines Teils des Militärregierungspersonals gegenüber der Entnazifizierung“ beklagt wurde.

Eine weitere wichtigte Aufgabe der Division lag in der Überwachung der Internierungslager für schwer belastete Nationalsozialisten, die unter die Automatic Arrest Category $^{32 t}$ fielen. Hatten in Hessen zunächst drei Lager (Civil Internment Enclosures) bestanden (C.I.E Nr. 73 in Ziegenhain, C. I. E. Nr. 75 in Schwarzenborn, C. I.E. Nr. 99 in Butzbach), so wurden diese im Frühjahr 1946 im Lager Darmstadt (C. I.E. Nr.91) zusammengefaßt. Die Bewachung von C.I.E. Nr.91 lag in den Händen des

314 OMGUS: Report of the Military Governor (1. April 1947 - 30. April 1948), Nr.34, Denazification, S.2.

315 OMGHE: Historical Report October 1945 - June 1946, Appendix I.

${ }^{316}$ Ebenda, Befehl vom 26.12.1945.

317 OMGHE: Historical Report October 1945 - June 1946.

${ }^{318}$ Ebenda; Text des Gesetzes in OMGUS: Report of the Military Governor (1. April 1947 30. April 1948), Nr. 34, Denazification, S. 52.

319 Ebenda, S.3.

$3208 / 216-3 / 8$

${ }^{321}$ Vgl. Directive JCS 1067 vom 26.4.1945, Abs. 8, OMGUS-Report of the Military Governor (1. April 1947 - 30. April 1948), Nr. 34, Denazification, S. 15 f. und spätere Bestimmungen, ebenda. 
39 th Field Artillery Battalion; das Geheimdienstpersonal stellte das 326th Headquarters Intelligence Detachment. ${ }^{322}$

Nach der Zusammenlegung der Lager in Darmstadt umfaßte C. I.E. Nr.91 23000 Insassen, deren Zahl sich in den folgenden Monaten aufgrund von Veränderungen in den Automatic Arrest Categories spürbar verringerte. Über Entlassungen entschieden die Security Review Boards der Militärregierung, die an die Stelle des am 15. Februar 1946 aufgelösten Security Review Board der 7.US-Armee getreten waren. ${ }^{323}$ Die Zahl der Insassen betrug am 1. November 1946 nur noch 11000 Personen. ${ }^{324}$ Auf Anordnung von USFET vom 13. Juli 1946 wurden Kontrolle und Bewachung des Lagers an das hessische Ministerium für politische Befreiung übergeben. ${ }^{325} \mathrm{Am} \mathrm{19}$. März 1947 verließ der letzte amerikanische Soldat das Lager, in dem noch immer $11000 \mathrm{Be}$ lastete einsaßen. ${ }^{326}$

Im Laufe des Jahres 1947 beobachteten die Amerikaner mit Sorge die Schwierigkeiten der Deutschen, geeignetes Personal für die Besetzung der Spruchkammern zu finden. ${ }^{327}$ Diese Probleme fanden auch darin ihren Ausdruck, daB im Sommer 1947 die KPD-Mitglieder unter Protest aus den Spruchkammern ausschieden. Die Enforcement Branch der Division beklagte die schleppende Durchführung der Entnazifizierung auf deutscher Seite. Dieses Drängen nach schnellerer Erledigung der Verfahren ging jedoch mit einer merklich nachlassenden Härte gegenüber den NS-Belasteten einher: So kamen in der zweiten Hälfte 1947 über 70000 Überprufte in den GenuB einer Amnestie; im Lager Darmstadt verringerte sich die Zahl der Insassen auf $6875{ }^{328}$

Die zunächst umfassend angelegte Entnazifizierungskampagne reduzierte sich zunehmend auf Einzelfälle: So wurde Ende 1947 das Verfahren gegen eine Gruppe früherer deutscher Generalstabsoffiziere in Neustadt (Hessen) vorbereitet, die zuvor bei der Historical Division der US-Armee zum Schreiben einer Geschichte der deutschen Beteiligung am Zweiten Weltkrieg angestellt waren. ${ }^{329}$

Da OMGHE die Entnazifizierung im Sommer 1948 als im wesentlichen abgeschlossen ansah, wurde die Denazification Division zum 1.Oktober 1948 aufgelöst. Einen Teil der verbliebenen Aufgaben fuhrte die in der Civil Administration Division gebildete Denazification Branch durch, während die Tätigkeiten der früheren Denazification Division, die über die Entnazifizierung hinausgingen, jetzt von der neu geschaffenen Investigation Division wahrgenommen wurden. ${ }^{330}$

\section{b) Organisation}

Die Organisationsstruktur der Denazification Division zeichnete sich durch eine starke Differenzierung aus: Diese Differenzierung und der damit zusammenhängende hohe Personalstand drückten die hohe Bedeutung aus, die die Amerikaner der Entnazifizierung in den ersten Jahren der Besatzung zumaßen.

\footnotetext{
322 OMGHE: Historical Report October 1945 - June 1946.

${ }^{323}$ Ebenda; vgl. auch oben B.19. Public Safety Division.

324 OMGHE: Historical Report July - December 1946.

$3258 / 188-2 / 6$.

$3268 / 190-2 / 11$.

327 OMGHE: Historical Report April - July 1947.

328 OMGHE: Historical Report July - September 1947.

329 OMGHE: Historical Report October - December 1947.

330 OMGHE: Historical Report 1948; General Orders Nr.11, OMGHE, 23.8.1948 und Nr.13, 23.8.1948 (8/179-3/4, 5/41-3/10); vgl. auch OMGUS-Hb, Teil Hessen B.23.
} 
Die Enforcement Branch überwachte die Vollstreckung der Spruchkammerurteile und verfolgte Mißachtungen des „Gesetzes zur Befreiung von Nationalsozialismus und Militarismus“. Ihre Investigation Section fahndete nach Fragebogenfälschungen. Die Fälschungen wurden dann von der Prosecution Section den Militärregierungsgerichten vorgelegt. Document Section archivierte die Berichte über Entnazifizierungslisten und sammelte Mitgliederlisten der NSDAP und anderes, für die Arbeit der Division wichtiges Material.

Civil Internment Camp Branch, die in der zweiten Jahreshälfte 1947 in Enclosure Supervision umbenannt wurde, war für die Überwachung der Internierungslager zuständig. Sie arbeitete dabei auch mit CIC zusammen und organisierte den Austausch von Internierten mit den anderen Besatzungszonen. Die angegliederte Camp Special Branch uberwachte die Spruchkammern im Lager Darmstadt.

Decision Review Branch wurde kurz nach Vorlage des ersten Organisationsüberblicks in zwei Sections aufgeteilt. Tribunal Decisions Section erhielt von den örtlichen Special Branches Problemfälle vorgelegt. Sie wies dann das Ministerium für politische Befreiung auf diese Probleme hin. Employment Eligibility Section uberprüfte die Mitglieder der Spruchkammern auf ihre demokratische Zuverlässigkeit. Wiesbaden Special Branch kontrollierte die deutschen Mitarbeiter der Militärregierung. Weiter überprufte sie die Entnazifizierungsverfahren der Inhaber von Spitzenpositionen in Politik und Wirtschaft.

Eine zentrale Rolle spielte die Field Supervision Branch. Sie hielt Kontakt mit dem Befreiungsministerium und überwachte die Einhaltung der Entnazifizierungsvorschriften auf allen Ebenen. Die Special Branch Officers der Liaison and Security Offices wurden von ihr beraten. Sie inspizierte die 47 hessischen Spruchkammern, die sieben Berufungskammern und die ihr angegliederten 41 Special Branches. Dazu waren acht, seit Ende 1947 nur noch sieben Area Supervisers eingesetzt.

Reports and Statistics war für das Berichtswesen zustăndig. Die Branch nahm von deutschen und amerikanischen Stellen Berichte entgegen und erstellte daraus Statistiken sowie die Berichte der Division.

Die Leitung der Division hatte die Executive Branch inne, die seit der zweiten Jahreshälfte 1947 aus dem Director und dem Deputy Director bestand.

Für Juni 1947 ergibt sich für die Denazification Division folgender Organisationsaufbau: Übersicht $66^{331}$

Diese Struktur blieb bis zur Auflösung der Division am 1. Oktober 1948 erhalten. Bereits am 23. August 1948 hatte General Order Nr.11 die Enforcement Branch der Division aufgelöst, aus der dann die Investigation Division gebildet wurde. ${ }^{332}$

\section{c) Personal}

Die Personalstärke dieser Division resultierte vor allem aus der hohen Zahl der deutschen Beschäftigten: Am 1.Juli 1946 arbeiteten 127 Deutsche in der Entnazifizierungsabteilung. Diese Zahl blieb im Jahr 1946 konstant und sank 1947 leicht (1. Januar: 125; 30.Oktober 1947: 110). Vor der Auflösung der Division am 1.Oktober 1948 kam es zu einem deutlichen Personalabbau (15. März: 105; 30.Juni 1948: 45). Die Zahl der amerikanischen Mitarbeiter stieg von 15 am 1.Juli 1946 auf 20 am 30 . Oktober 1947, um zum 30. Juni 1948 auf sieben abzusinken.

$3315 / 41-2 / 7$.

332 5/41-3/10. 
Ubersicht 66: Organisation der Denazification Division, OMGHE, 30. Juni 1947

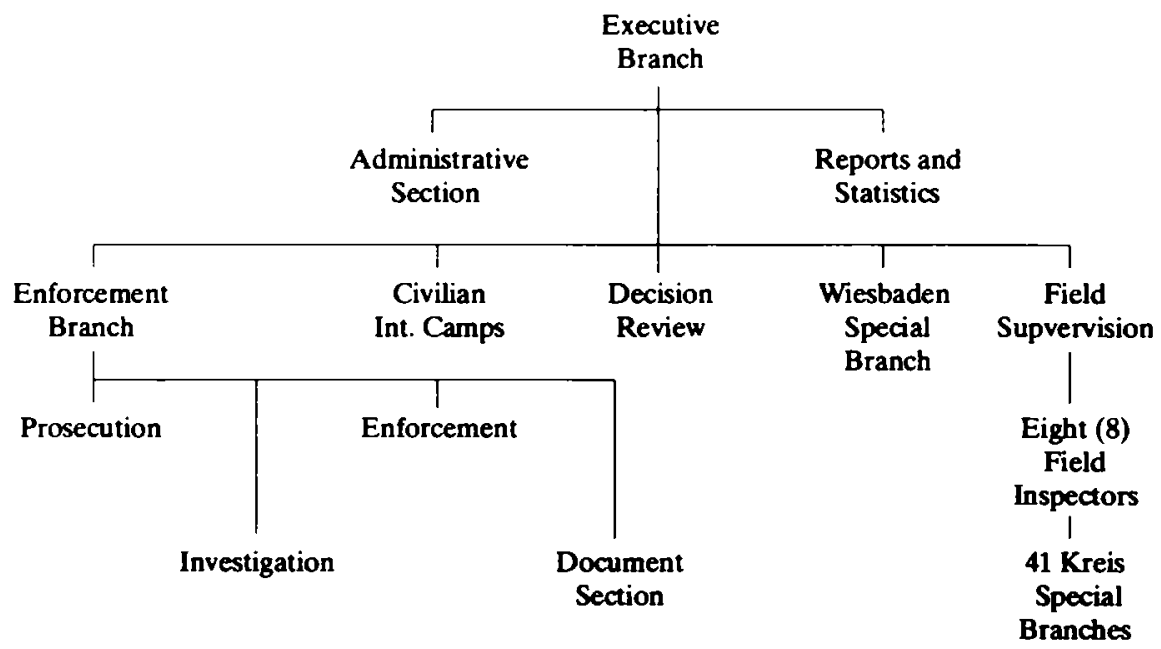

Die Division leitete seit ihrer Bildung Hubert J. Teitelbaum. Ihm stand Arsen L. Yakoubian als Deputy Chief zur Seite. Dieser wurde mit General Order Nr. 10 am 15. Dezember 1947 zum kommissarischen Leiter bestimmt. An seine Stelle trat, gleichfalls kommissarisch, Roger E. Reynolds. Während dieser bis zur Auflösung in der Division blieb, wechselte Yakoubian im Frühjahr zur Liaison and Security Control Division. Nach kurzer Abwesenheit arbeitete dann Teitelbaum wieder als Chief. Bei Auflösung der Division wurde er mit General Order Nr. 15 vom 23. August 1948 zum Chief der Denazification Branch in der Civil Administration Division ernannt, und am 1. Oktober 1948 auch zum Chief der Public Safety Branch (General Order Nr. 19). Am 23. August 1948 hatte ihn Newman außerdem mit General Order Nr. 12 zu seinem Denazification Adviser ernannt.

\section{Investigation Division}

\section{a) Aufgaben}

General Order Nr. 11 vom 23. August 1948 löste die Enforcement Branch in der Denazification Division auf und überführte Personal und Ausriłstung in die Investigation Division, die zum 1.September 1948 neu geschaffen wurde. Als Aufgaben der neuen Division legte der Befehl fest:

a) „Auf Anordnung des Direktors Untersuchung aller Angelegenheiten, die Personal, Ausrüstung, Aufgaben und Aktivitäten von OMGHE und seiner nachgeordneten Dienststellen betreffen;

b) Annahme und Untersuchung von Beschwerden der Bediensteten von OMGHE und seiner nachgeordneten Dienststellen;

c) Durchführung aller anderen Untersuchungen, die vom Direktor angefordert werden. “333

$3335 / 41-3 / 10$. 
Es handelte sich also um eine interne Kontrollbehörde.

b) Organisation und Personal

Die wenigen Beschäftigten der Division bearbeiteten die Bereiche Verwaltung, Untersuchungen und Berichte: $:^{334}$

Ubersicht 67: Organisation der Investigation Division, OMGHE, 31. Dezember 1948

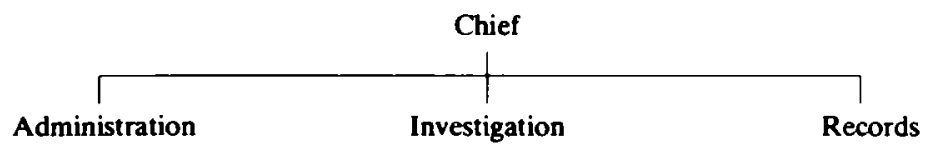

Zwei Wochen nach der Aufstellung der Division forderte OMGHE bei OMGUS drei zusätzliche Personalstellen für diesen Bereich an. ${ }^{335}$ Dieser Forderung war offenbar kein Erfolg beschieden: Zum Jahreswechsel waren nur zwei Amerikaner in der Division beschäftigt, die von zwölf deutschen Mitarbeitern unterstützt wurden. Am Ende der OMGHE-Amtszeit beschränkte sich das US-Personal der Division nur noch auf den Chief.

General Order Nr.14 vom 23. August 1948 hatte John A. Stark zum Chief der neuen Division ernannt. Ihm stand Philipp A. Damon zur Seite. Zuvor waren Stark als Investigator und Damon als Administrative Assistant in der Denazification Division tätig gewesen. Im Frühjahr 1949 wechselte Allan C. Wilson in die Division, wo er als Chief und einziger Amerikaner bis zum Ende ausharrte.

\section{Statistical, Historical and Field Reports Division}

\section{a) Aufgaben}

Schon während der Zeit, in der das Detachment E1A2 in Frankreich lag, begann der Historical and Political Information Officer Edgar L. Erickson mit seiner Arbeit. In französischen Archiven und Bibliotheken, in deutschen Hinterlassenschaften und bei den entsprechenden Abteilungen der US Army suchte und sammelte er Material über Deutschland. Seine besondere Aufmerksamkeit widmete er dem vorgesehenen Einsatzgebiet des Detachments, der Region Saar-Pfalz-Südhessen. In den War Diaries, die bis Mitte 1945 über die Aktivitäten des Detachments berichten, finden sich an vielen Stellen Meldungen über seine Funde von Unterlagen über die NSDAP oder von geographischen und historischen Informationen. ${ }^{336}$

Am 1. Juli 1945 wurde dann die Historical Information and Political Intelligence Division gebildet. Zu deren Aufgaben gehörten zunächst:

- die Vorbereitung und Abfassung der Monatsberichte E5/OMGHE (Historical Reports),

- die Kontrolle des Berichtswesens der Detachments (Field Reports),

- der Bereich Denkmäler, Kunst und Archive (Monuments, Fine Arts \& Archives),

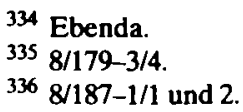


- politische Überwachungsmaßnahmen und

- Aufklärungsaufgaben (Intelligence).

Der Bereich Monuments, Fine Arts \& Archives (MFAA) wurde im Herbst 1945 der Finance Division zugeordnet - der Beginn einer Odyssee durch verschiedene Divisions. Die politischen Überwachungsaufgaben übernahm zum Jahresende die Civil Administration Division. Mit der Bildung der Intelligence Division übernahm diese auch alle Aufgaben im Aufklärungsbereich. Als Tätigkeitsbereiche verblieben so die Vorbereitung und Erstellung der Historical Reports und die Aufsicht über die Field Reports. Anfang 1946 wurde der gesamte Bereich der Statistik (Statistical Investigation) in die Verantwortung der Division gestellt und überschattete bald alle anderen Aufgaben. Die entsprechende Branch der Division koordinierte den Aufbau des Hessischen Statistischen Landesamtes, das am 14. Januar 1946 auf Grundlage der Organizational Direction Nr.15, OMGHE, unter Aufsicht der Division seine Tàtigkeit aufgenommen hatte. Im zweiten Arbeitsbereich der Division, den Field Reports, herrschte im Herbst 1945 ein riesiges Durcheinander. Eine USFET-Direktive von Ende Oktober hatte sich die Organisation und Kontrolle des gesamten Berichtswesens der Militärregierung zum Ziel gesetzt. Die darin vorgesehenen Dienstwege bildeten jedoch nach Meinung der Division ein „gespenstisches Labyrinth“, wie es im Historical Report vom Frühjahr 1946 heißt. „Diese Konfusion“, fährt der Bericht fort, „führte zu einem unglücklichen Verfahren: In erster Linie wurde danach getrachtet, die Berichte rechtzeitig hereinzubekommen, während nur wenige Gedanken auf ihren Inhalt verwendet wurden. ${ }^{\text {337 }}$ Acht Monate muBten vergehen, bis man (im Juni 1946) daran ging, den Berichtswirrwarr zu entwirren. Hatten die Detachments zunächst mindestens dreiBig Berichte pro Monat abliefern müssen, so konnten sich die Liaison \& Security Offices jetzt mit sechs bis acht begnügen. Entlastung brachte auch die Verringerung der vorgesetzten Stellen im Armee- und OMGUS-Bereich. Seit Frühjahr 1946 entfielen dann bei den Kreisund Regierungsbezirkdetachments die Field Reports and Historical Functions. Jetzt wurden deutsche Behörden zu dieser allgemeinen Berichterstattung herangezogen. Zur Abfassung der Historical Reports wurde im März 1946 ein "full-time historian“ eingestellt. Seit 1946 erschienen die Reports vierteljährlich.

Daneben war die Division damit beauftragt, die von OMGHE veröffentlichten Anordnungen zu verteilen und zu archivieren. Im März 1946 wurde die ursprüngliche Bezeichnung der Division in Historical and Field Reports Division geändert. Im Juli 1946 erfolgte eine erneute Umbenennung, als ein Statistiker zum Chef der Division ernannt wurde. Ihre Bezeichnung lautete von nun an Statistical, Historical and Field Reports Division. Ernennung und Namensänderung waren äuBerliche Zeichen für die Verschiebung der Aufgabenschwerpunkte der Division weg vom Berichtswesen hin zur Statistik.

Der neue Chef versuchte denn auch bald, die Zuverlässigkeit der Datenerhebung zu verbessern. So drängte er die Landesregierung zur Einsetzung eines ständigen Koordinierungsausschusses für Statistik sowie zur Ernennung von Statistikern auf Kreisund Regierungsbezirksebene. Durch Field Inspectors ließ er das deutsche Berichtswesen systematisch überprüfen und verbesserte die Formblätter zur Datenerhebung. Offenbar hatte er mit seinen Maßnahmen Erfolg: OMGUS wies die anderen Ländermilitärregierungen an, ähnliche Maßnahmen zu ergreifen.

Im Februar 1947 hatte die Division alle Gliederungen und Aktiväten der Militärregierung und der hessischen Landesregierung sowie deren Beziehungen untereinander in

337 OMGHE: Historical Report April - July 1947. 
übersichtlichen Darstellungen erfaBt und im Hauptquartier ausgestellt, damit sich der „schnelle Besucher" mit den Aufgaben und Aktivitäten der Militärregierung vertraut machen konnte. Die graphischen Darstellungen der Organisation der Militärregierung (Organizational Charts) wurden seitdem den Historical Reports beigefügt. Der statistische Teil der Reports wurde systematisiert und der Umfang des „erzählenden“ Teils auf 150 Seiten je Vierteljahresbericht beschränkt. An die Reports wurden Pressemitteilungen und Fotographien angefügt. ${ }^{338}$

Der fruhere Reporter der Armee-Zeitung „Stars and Stripes“ Dexter L. Freeman verfaBte 1947/48 als Mitarbeiter der Division das Buch „Hesse - a new German state“, das im Sommer 1948 erschien und die Arbeit der amerikanischen Militärregierung und ihren Beitrag zur Bildung des Landes Hessen darstellen sollte. ${ }^{339}$ Die statistische Arbeit, vor allem im wirtschaftlichen Bereich, erweiterte sich Anfang 1948 hin zu Auswertung und Analyse. Seit diesem Zeitpunkt wurden auch wieder Monatsberichte für OMGHE angefertigt. 340

\section{b) Organisation}

Nach den beschriebenen Veränderungen Ende 1945/Anfang 1946 hatte die Division Mitte 1946 folgende Organisationsstruktur: $:^{341}$

Ubersicht 68: Organisation der Statistical, Field Reports and Historical Division, OMGHE, Juli 1946

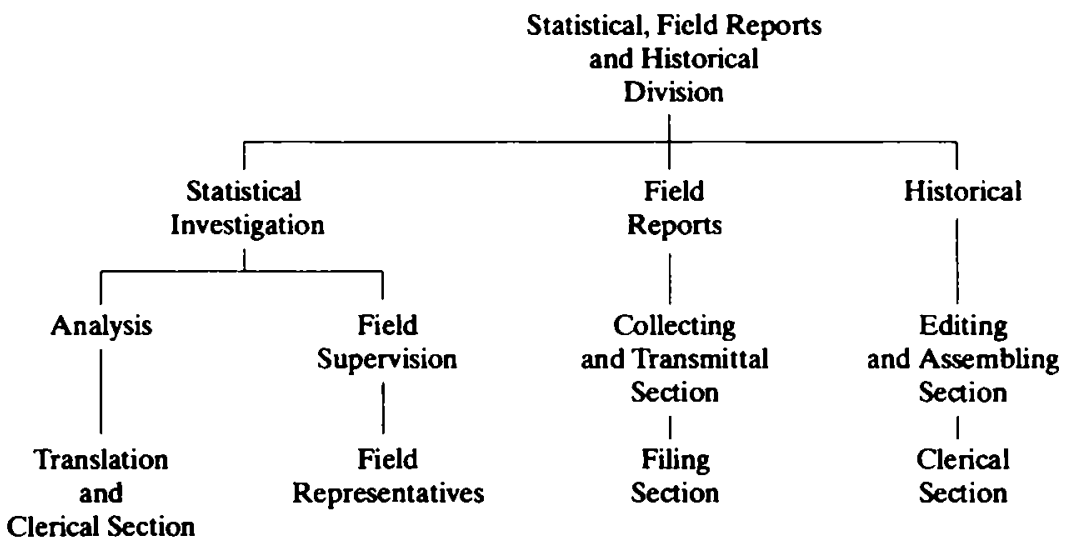

Die Aufteilung der drei Branches in acht Sections, die angesichts der zumeist nur vier amerikanischen Mitarbeiter der Division bereits 1946 nur auf dem Papier bestand, wurde 1947 fallen gelassen.

Anfang 1948 wurde für den Bereich Statistical Analysis eine eigene Branch eingerichtet, so daB die Division im letzten Jahr ihrer Tătigkeit folgende Organisationsstruktur hatte: ${ }^{342}$

\footnotetext{
338 Ebenda.

339 Freeman: Hesse. A New German State. o. O. o.J. (Frankfurt 1948).

340 5/41-2/17.

341 OMGHE: Historical Report October 1945 - June 1946.

$3425 / 41-2 / 17$.
} 
Ubersicht 69: Organisation der Statistical, Historical and Field Reports Division, OMGHE, 31. März 1948

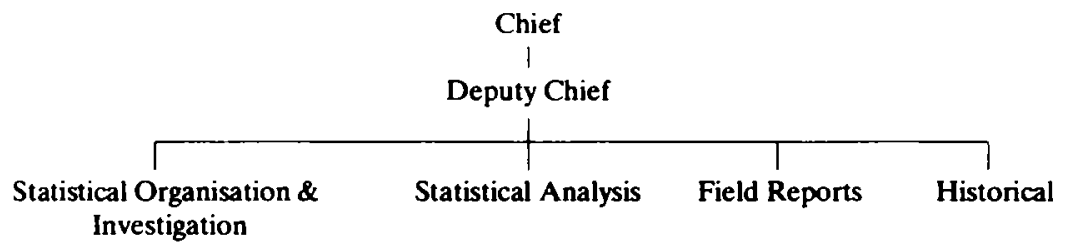

Im Frühjahr 1948 entfiel die Stelle des Deputy Chief - es waren ohnehin nur noch vier Amerikaner in der Division tätig.

c) Personal

Die Statistical, Historical and Field Reports Division gehörte zu den kleineren Divisions von OMGHE. Sie hatte zunächst vier amerikanische Mitarbeiter. Ende 1947 kam eine Stelle dazu, die aber im Frühjahr 1948 wieder entfiel. Der amerikanische Personalbestand sank dann kontinuierlich bis auf eine Position - nämlich die Stelle des Division Chief, der vom 16. März 1949 bis zum Ende der Tătigkeit von OMGHE als einziger Amerikaner in der Division verblieb. Nachdem zunächst nur zwei Deutsche in der Division arbeiteten, erhöhte sich deren Zahl im Zusammenhang mit den vermehrten Aufgaben im statistischen Bereich bis auf 20 am 1. Januar 1947. Die deutsche Personalstärke bewegte sich $1947 / 48$ zwischen 15 und 20 , um dann gleichfalls zu fallen.

Robert A. Irving, der bereits in der Zeit, als E-5 die Militärregierung in Neustadt gestellt hatte, Leiter der Division gewesen war, behielt diese Position bis zum Juli 1946. Dann wurde er von dem gelernten Statistiker Donald M. Marvin abgelöst: Die Division hatte jetzt vor allem statistische Aufgaben wahrzunehmen. Nach Marvin leitete Clifford K. Burke die Division. Burke war zunächst in der Economics Division tătig gewesen. Seine Dienstzeit dauerte von Februar bis Juni 1948. Dann ruckte Robert A. Irving wieder in die Leitungsposition auf, die er bis zum Ende von OMGHE innehatte.

\section{Transportation Division}

\section{a) Aufgaben}

Die Transportation Division war für den Eisenbahn-, Straßen- und Schiffsverkehr in Hessen sowie für die entsprechenden Verkehrswege zustăndig. Das zivile Verkehrssystem war im Mai 1945 völlig zusammengebrochen. Zunächst hatten sich die örtlichen Detachments dieser Probleme anzunehmen. Sie setzten dafür auf Ortsebene „Fahrbereitschaftsleiter" und auf Regierungsbezirksebene „Gruppenfahrbereitschaftsleiter" ein, die den Militärregierungsstellen gegenüber für die Verwaltung des Mangels verantwortlich waren.

Vor allem im Bahnbereich suchten die Transportoffiziere der Militärregierung die Zusammenarbeit mit dem Military Railway Service der US-Armee. Zu diesem Zweck fand am 8. August 1945 in Frankfurt ein Treffen zwischen Vertretern der Militärregierung und des Military Railway Service statt. Auf dem Treffen kam man überein, die 
Bahnlinien der Reichsbahndirektionen Frankfurt und Kassel, die das Gebiet des späteren Landes Hessen umfaßten, in zwei Kategorien einzuteilen. Kategorie I sollte vorrangig dem militärischen Bedarf dienen, während Kategorie II für deutsche Transportbedürfnisse zur Verfügung stand.

Im Herbst stellte die inzwischen gebildete Militärregierung eine Prioritätenliste für die Güterbeförderung auf:

1. Lebensmittel, landwirtschaftliche Guter und landwirtschaftlicher Bedarf,

2. Kohle und Treibstoff, der zur Beförderung von Lebensmitteln benötigt wird,

3. Brennholz und

4. Baumaterialien.

Die Division konnte nach und nach die direkte Kontrolle über das Transportwesen an deutsche Stellen abgeben, die zum großen Teil im hessischen Ministerium für Wirtschaft und Verkehr zusammengefaBt waren. So wurde im November 1945 die hessische Wasserstraßendirektion gebildet, die zunächst der Staatskanzlei unterstand. Die Straßenbauämter wurden zum 10. Oktober 1945 reorganisiert und am 1. Januar 1946 unter deutsche Kontrolle gestellt. Die Straßenmeistereien und der Bevollmächtigte für den Nahverkehr arbeiteten unter direkter Kontrolle der Militärregierung, bis sie am 12. April 1946 dem Verkehrsministerium unterstellt wurden. Im Sommer 1945 war die Entnazifizierung der hessischen Reichsbahnmitarbeiter angelaufen, die nach Meinung der Transportation Division vor allem im Winter 1945/46 die Leistungsfähigkeit der Eisenbahn beeinträchtigte.

Die Armee, die im Herbst 1945 und 1946 auch beim Einfahren der Ernte half, stellte bis Juli 1946 den Deutschen über die Militärregierung 2800 defekte, aber reparable Fahrzeuge und 3036 Anhänger zur Verfügung. Die Reparaturarbeiten und die teilweise Umristung auf Holzvergaser wurden durch hessische Firmen in Kassel und Wiesbaden ausgeführt. ${ }^{343}$

Der harte Winter 1946/47 brachte auch in Hessen einen weitgehenden Zusammenbruch des Verkehrswesens. ${ }^{344}$ Ein chronisches Problem war der Mangel an Reifen. Aus diesem Grund standen im Sommer 1947 zeitweise 2558 Lastwagen still. ${ }^{345}$ Bei diesen und anderen Materialproblemen arbeitete die Transportation Division eng mit der Economics Division zusammen. Seit Sommer 1947 gab sich die Division jedoch optimistisch, was die Lösung der Verkehrsprobleme betraf. ${ }^{346}$ Bei der Eisenbahn konnte bis Ende 1948 der Mangel an Lokomotiven behoben werden. Dazu trugen vor allem die Kasseler Henschel-Werke bei, die bis zu 32 Lokomotiven im Monat reparierten.

Einen Einschnitt brachte die Währungsreform im Juni 1948. Die Personenbeförderung der Eisenbahn sank auf dreißig Prozent des Standes vor der Reform. Ende 1948 hatte er wieder fünfzig Prozent des vorherigen Standes erreicht. Auf der anderen Seite nahm nach der Währungsreform der Autoverkehr zu. ${ }^{347}$

Die Division war auch für den Director's Special Diesel Train (Dieselsonderzug) zuständig. Mit diesem Zug (Train Nr. 137.448) fuhrte J. R. Newman seine Reisen durch. Der Zug stand zudem den Division Chiefs für ihre Dienstreisen zur Verfügung. ${ }^{348}$

\footnotetext{
343 OMGHE: Historical Report October 1945 - June 1946; 8/189-1/5.

344 8/190-2/11; OMGHE: Historical Report July - September 1947.

345 OMGHE: Historical Report April - July 1947.

346 OMGHE: Historical Report July - September 1947.

347 17/19-2/8; OMGHE: Historical Report 1948.

348 17/19-2/8; OMGHE: Historical Report July - September 1947.
} 


\section{b) Organisation}

Die Division war während des gesamten Zeitraums ihres Bestehens in vier Branches aufgeteilt:

- Railroads war für die Eisenbahnen und für die Verbindung zwischen OMGHE und Reichsbahn zuständig.

- Vehicle Maintenance beschäftigte sich mit allen, die deutschen Straßenfahrzeuge betreffenden Problemen. Die Branch führte darüber für OMGUS Statistiken.

- Road Operations überwachte die Einhaltung der Militärregierungsvorschriften für den Straßenverkehr durch deutsche Stellen.

- Road and Bridge Construction \& Maintenance überwachte die deutsche Straßenbauverwaltung.

Im Juni 1946 stellte sich die Organisation der Transportation Division wie folgt dar: $:^{349}$

Ubersicht 70: Organisation der Transportation Division, OMGHE, 30. Juni 1946

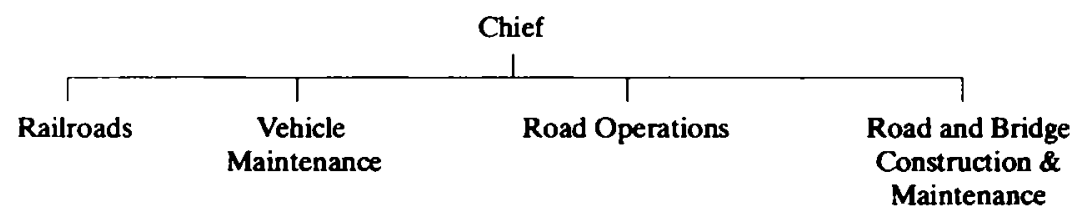

Für den Dieselsonderzug war der Chief of Division verantwortlich. Nach der Auflösung der Division wurde der für den Zug zuständige Mitarbeiter - der „Superviser of Diesel“" Albert Filupeit - Command and Executive zugeordnet. Railroads wurde später in Inland Waterways and Railroads umbenannt, die Branch war auch für die Verbindung mit der hessischen Wasserstraßenverwaltung zuständig. In dieser Form arbeitete die Division bis zu ihrer Auflösung durch General Order Nr. 5 vom 20. April 1948. Die Anordnung bestimmte, daß die Aufgaben der Transportation Division von diesem Zeitpunkt an von der Transportation Branch in der neugebildeten Bizonal Liaison Division wahrgenommen werden sollten. Mit General Order Nr. 19 vom 1. Oktober 1948 wurde der Chief of Branch zum Transportation Adviser to the Director ernannt. Im Rahmen der Auflösung der Bizonal Liaison Division fiel die Transportation Branch ganz weg.

\section{c) Personal}

Trotz der zentralen Bedeutung, die das Transportwesen für den wirtschaftlichen Wiederaufbau hatte, gehörte die Transportation Division immer zu den kleinsten Divisions von OMGHE. Im Jahr 1946 arbeiteten vier Amerikaner in der Division, Anfang 1947 nur drei. Am 16. Oktober 1947 erhielt die Division von OMGUS zusätzlich einen Administrative Assistant zugewiesen, so daß vorubergehend wieder vier Amerikaner in der Division arbeiteten. Am Jahresanfang 1948 zählte die Transportation Division wieder drei, im März - kurz vor ihrer Auflösung - vier amerikanische Mitarbeiter. In der Transportation Branch der Bipartite Liaison Division arbeiteten zunächst drei Amerikaner aus der früheren Transportation Division; im Jahr 1949 blieb nur einer von ihnen, der Chief of Branch, übrig. Die Zahl der deutschen Mitarbeiter der Division sank kontinuierlich von fünf 1946 auf drei vor Auflösung der Division.

$3495 / 41-27$. 
Chief der Division war von Juli 1945 bis August 1947 Charles F. Hennessy, der schon bei der Aufstellung von E1A2 als 1st Lt. (später Capt.) in Rochefort dabei gewesen war. Im August 1947 schied Hennessy gemeinsam mit Dale T. Barker, dem Chief of Road Supply Branch, aus der Division aus. Vom 1. September bis 31. Dezember 1947 leitete der Deutsche Kurt Magnus als „Emergency Overall Director“ kommissarisch die Division. Für ihn kam Anfang 1948 Theodore E. Dodds, der die Division bis zu ihrer Auflösung leitete und dann als Chief of Transportation Branch in der Bipartite Liaison Division sowie als Transportation Adviser tätig war 


\section{Quellenbeschreibung}

\section{Die Akten der amerikanischen Militärregierung in Hessen}

Die in den hessischen Staatsarchiven Wiesbaden, Darmstadt und Marburg zur Verfügung stehenden Aktenbestände der amerikanischen Militärregierung für Hessen (OMGHE) sind ein verfilmter Teilbestand der im Washington National Record Center (einer Außenstelle des Nationalarchivs in Suitland, Maryland) als Record Group 260 (RG 260) aufbewahrten Originalakten des „Office of Military Government for Germany, United States“ (OMGUS).

Bei dem OMGHE-Teil des Bestandes RG 260 handelt es sich aber auch in anderer Hinsicht um einen Teilbestand. Zum einen sind die Akten wohl schon bei der Verschiffung nach Amerika nicht mehr ganz vollständig gewesen; bestimmte Teile der Akten (vor allem Personalakten) sind von den Amerikanern nicht freigegeben worden; weitere Lücken dürften ihre Ursachen in Transportverlusten haben, denn auch auf den Verladelisten (Shipping Lists) noch aufgeführte Archivboxen sind nicht mehr vorhanden. Zum anderen sind von den deutschen Bearbeitern bei ihrer Verzeichnungsaktion weitere Einschränkungen vorgenommen worden:

1. Sogenannte „Folders of no historical value“ - also als archivunwürdig angesehene Akteneinheiten -, die in der Bundesrepublik der Kassation anheim gefallen wären, wurden nicht verfilmt. Sie sind mit Kurztiteln auf gelben Erfassungsbögen erfaBt und am Ende der Verzeichnung einer Organisationseinheit angehängt.

2. Akteneinheiten, die nicht als völlig wertlos angesehen wurden, bei denen aber die Wahrscheinlichkeit der Doppeluberlieferung bestand, es sich um Mehrfachexemplare handelte oder wo das Original von so schlechter Qualität war, daB eine Verfilmung zwecklos erschien, wurden zwar näher beschrieben, aber nicht verfilmt. Insgesamt wird der Anteil des letztlich verfilmten Aktenmaterials nach Angaben von Bearbeitern ${ }^{1}$ auf 30 bis $50 \%$ geschätzt.

Die ErschlieBung des OMGHE-Bestandes ist bis jetzt nur über die Erfassungsbögen (data sheets), die von den deutschen Archivaren angelegt wurden, möglich. Auf ihnen sind durch die ausfuhrliche Beschreibung des Inhalts der einzelnen Faszikel (folder), durch die Provenienzangabe, den erfaßten Zeitraum sowie Angaben uber Größenordnung, Entnahmen und Bearbeiter die wesentlichen Informationen enthalten. Geordnet sind diese Verzeichnungsblätter allerdings nach Provenienzen, denen die Organisationsstruktur von OMGHE in ihrer Endphase zugrunde liegt.

Da aber die Binnenstruktur von OMGHE häufig wechselte und damit auch Registraturzusammenhänge vernichtet wurden, ist das Auffinden einzelnder Akteneinheiten häufig mit großem Zeitaufwand verbunden.

Eine denkbare Rekonstruktion von Aktenserien anhand der Aktenzeichen ist leider kein gangbarer Weg, da nur ein Teil der Akten uberhaupt mit Aktenzeichen versehen ist. Mahnungen von OMGHE an ihre nachgeordneten Dienststellen, das von diesen für ihre Zwecke als unbrauchbar erachtete „Dewey Decimal File System“ doch flexi-

${ }^{1}$ Bei diesen Angaben stützen wir uns auf Aussagen deutscher Archivare, die an der Verzeichnung der Akten mitgewirkt haben. Es gaben uns freundlicherweise Auskünte die Herren E. G. Franz und A. Hildebrandt (Staatsarchiv Darmstadt), G. Menk (Staatsarchiv Marburg), H.-J. Haebel und B. Helfer (Hauptstaatsarchiv Wiesbaden) und H.G. Ruppel (Stadtarchiv Offenbach). 
bler zu handhaben und für die einheitliche Kennzeichnung der anfallenden Akten anzuwenden, belegen dies. ${ }^{2}$

Angesichts dieser Probleme wäre eine mit Hilfe der Elektronischen Datenverarbeitung noch zu leistende Indexierung des Bestandes ein Meilenstein in seiner Benutzbarkeit. Gleichzeitig könnten durch Vernetzung der Indices teilweise Lücken in den Länderbeständen geschlossen werden. ${ }^{3}$ Das wird wohl noch für geraume Zeit Zukunftsmusik bleiben.

Um nicht von der - trotz aller Einschränkungen - immer noch enormen Fülle des Aktenmaterials erschlagen zu werden, sollte der interessierte Benutzer zweckmäBigerweise immer mit den Historical Reports seinen Einstieg in die Arbeit suchen. Reports wurden auf allen Ebenen der Militärregierung (Divisions, Branches, Detachments) in regelmäBigen Zeitabständen erstellt und wurden durch die Historical Division von OMGHE zu umfangreichen Historical Reports (meist mit mehreren Anhängen) zusammengestellt.

Angesichts der Probleme bei der Erschließung des Aktenmaterials und der Lücken im OMGHE-Bestand geben wir eine Übersicht mit Angabe der Fundstellen über die Historical Reports von OMGHE. Die Historical Reports einzelner Divisions oder Branches finden sich am konzentriertesten sowohl im OMGHE-Bestand Historical Division (8/187-1 bis 8/191-2) als auch des Control Office, Historical Division, OMGUS.

Historical Reports des Office of Military Government for Hesse (Greater Hesse)

Historical Report October 1945 - Juni 1946 (4 Volumes)

$\begin{array}{llll}\text { Vol. I } & \text { Narrative } & 8 / 190-2 / 9 & 5 / 41-1 / 10 \\ \text { Vol. II } & \text { Appendix I } & 8 / 190-2 / 10 & 5 / 41-1 / 20 \\ \text { Vol. III } & \text { Appendix II } & 5 / 41-1 / 21 \\ & \text { Organization Plans and Charts } & \\ \text { Vol. IV } & \text { Appendix III Pictorial } & 5 / 41-1 / 22\end{array}$

Historical Report July 1946 - December 1946 (3 Volumes)

Vol. I Narrative 5/41-2/1

Vol. II Appendix 5/41-2/2

Vol. III Pictorial 5/41-2/3

Historical Report January - March 1947 (3 Volumes)

$\begin{array}{llll}\text { Vol. I } & \text { Narrative } & 8 / 190-2 / 11 & 5 / 41-2 / 4 \\ \text { Vol. II } & \text { Appendix } & 8 / 190-2 / 12 & 5 / 41-2 / 5 \\ \text { Vol. III } & \text { Pictorial } & & 5 / 41-2 / 6\end{array}$

Historical Report April - June 1947 (3 Volumes)

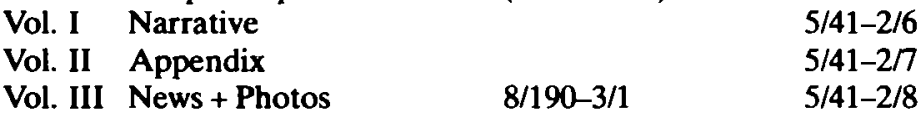

2 8/216-3/9.

${ }^{3}$ Pionierarbeit hat in diesem Zusammenhang schon das Archiv des Instituts für Zeitgeschichte in München mit der Verzeichnung des zentralen OMGUS-Bestandes geleistet, in dem sich zahlreiche Akten befinden, die sich auf die Länder und die einzelnen Ländermilitärregierungen beziehen. 
Historical Report July - September 1947 (4 Volumes)

Vol. I Narrative

$5 / 41-2 / 9$

Vol. II Appendix

$8 / 190-3 / 2$

$5 / 41-2 / 10$

Vol. III News and Photographic Supplement

$5 / 41-2 / 11$

Vol. IV Special Intelligence Appendices

$5 / 41-2 / 12$

Historical Report October - December 1947 (3 Volumes)

Vol. I Narrative 8/190-3/3

Vol. II Appendix 8/190-3/3 5/41-2/14

Vol. III News and Photo Supplement

$5 / 41-2 / 15$

Historical Report 1948 (3 Volumes)

Vol. I Narrative

5/41-3/9

Vol. II Appendix

$3 / 437-1 / 4$

$5 / 41-3 / 10$

Vol. III News and Photographic 3/437-1/1

$5 / 41-3 / 12$

Supplement

Historical Report January - March 1948 (4 Volumes)

Vol. I Narrative

5/41-2/16

Vol. II Appendix

$3 / 437-1 / 9$

5/41-2/17

Vol. III News and Photographic 3/437-1/6

$5 / 41-2 / 18$

Supplement

Vol. IV Special Intelligence Appendices

$5 / 41-2 / 19$

Historical Report Hesse-Nassau, September 1945

(1 Volume)

$5 / 41-3 / 1$

Historical Report October 1945 (2 Volumes)

Vol. I History

3/437-1/7

$5 / 42-1 / 2$

Vol. II Appendix

$3 / 437-1 / 8$

$5 / 41-3 / 2$

Historical Report November 1945 (2 Volumes)

Vol. I History

Vol. II Appendix

$5 / 41-3 / 3$

$5 / 41-3 / 4$

Historical Report December 1945 (2 Volumes)

Vol. I History

$5 / 41-3 / 5$

Vol. II Appendix

$5 / 41-3 / 6$

Historical Report January 1946 (2 Volumes)

Vol. I History

$5 / 41-3 / 7$

Vol. II Appendix

$5 / 41-3 / 8$

Historical Report February 1946 (2 Volumes)

Vol. I Narrative

Vol. II Appendix

Report November - December 1947

$5 / 42-3 / 4$

Report January - March 1948

$5 / 42-3 / 5$

Report April - June 1948

$5 / 42-3 / 6$

Report July - September 1948

$5 / 42-3 / 7$ 
Report October - December 1948

Report January - February 1949

Report May - June 1949

Report July - September 1949

Semi-monthly Reports June - November 1947
$5 / 42-3 / 8$

$5 / 42-3 / 9$

$5 / 42-3 / 10$

$5 / 42-3 / 11$

$5 / 42-3 / 3$

Der OMGHE-Bestand bietet aber auch lokal- und regionalhistorisch interessantes Material in Hülle und Fülle.

Für die Anfangsphase der Militärregierung finden sich die Berichte der Lokal-, Kreisund später Regierungsbezirks-Detachments am dichtesten bei der Historical Division und für später in der Abteilung Residence Office (Wiesbaden, Frankfurt, Darmstadt, Marburg, Bad Hersfeld, Kassel). Da dieser Bestand ebenfalls Lucken aufweist, empfiehlt es sich wiederum, zur Ergänzung den Bestand Control Office, Historical Division, OMGUS, heranzuziehen. Für eine erste Orientierung geben wir für die vielfältige Reportliteratur der Detachments (Orte in alphabetischer Reihenfolge) die Fundstellen an und geben zu den einzelnen Abschnitten des Repertoriums des OMGHE-Bestandes (HStA Wiesbaden) kommentierende Stichworte.

Fundstellenverzeichnis für die von den Detachments (Liaison \& Security Offices auch im Repertorium unter Residence Office) erstellten Reports (Tages-, Wochen-, Monatsberichte; Historical, Political, Intelligence Reports)

A. Regional Detachments

Darmstadt E-3 (E1B2)

8/191-1/1

Frankfurt E-6 (E1D2)

8/189-3/17

8/194-2/7

8/194-3/1

$5 / 8-1 / 1$

$5 / 8-1 / 7$

Kassel E-4 (E1C2)

8/187-1/3

$8 / 187-2 / 1$

$8 / 187-2 / 4$

$8 / 187-2 / 7$

8/187-2/10

8/189-3/8

$5 / 7-3 / 8$

$5 / 9-2 / 6$

Marburg E-2 (E1G2)

8/93-1/9

$5 / 7-2 / 3$
$5 / 7-3 / 2$

8/191-2/19

8/194-2/8

$8 / 194-3 / 3$

$5 / 8-1 / 2$

$8 / 187-1 / 4$

$8 / 187-2 / 2$

$8 / 187-2 / 5$

$8 / 187-2 / 8$

$8 / 187-2 / 11$

$5 / 7-2 / 6$

$5 / 7-3 / 9$

$8 / 190-3 / 6$

$5 / 9-2 / 4$
$5 / 7-3 / 4-7$

8/194-2/6

$8 / 194-2 / 9$

8/194-3/4

$5 / 8-1 / 5$

$8 / 187-1 / 5$

$8 / 187-2 / 3$

$8 / 187-2 / 6$

8/187-2/9

8/189-2/12

$5 / 7-3 / 1$

$5 / 9-2 / 4$

8/191-2/14

$5 / 9-2 / 6$

Wiesbaden E-5 (E1A2) (Historical Reports siehe oben)

$8 / 187-1 / 2$

8/189-3/14

8/189-3/12

$8 / 190-3 / 9$

$8 / 189-3 / 13$

8/194-2/1

8/194-2/3

8/194-2/4 
$8 / 194-2 / 5$

$8 / 194-3 / 6$

$5 / 8-1 / 2$

B. Local Detachments

Alsfeld H-59 (19D2)
$8 / 189-3 / 7$

$5 / 9-1 / 6$

Arolsen I-142 (I5G2)

5/9-3/9

Bad Homburg H-86 (L2D2)

8/191-1/11

$8 / 191-2 / 10$

$5 / 9-3 / 11$

Bad Orb I-145 (I17G2)

5/9-3/10

$8 / 194-2 / 10$

$8 / 195-1 / 2$

$5 / 8-1 / 3$

$8 / 194-3 / 5$

5/8-1/1

$5 / 8-1 / 4$

$8 / 191-2 / 3$

$5 / 9-1 / 3$

Bad Schwalbach I-148 (I3D2)

8/191-1/7

$5 / 9-3 / 14$

Beerfelden 1-140 (I1B2)

5/9-3/8

Bensheim/Heppenheim G-31 (I6B2)

8/191-2/2

$8 / 191-2 / 5$

$5 / 9-3 / 7$

$5 / 8-2 / 4$

Biedenkopf H-76 (I4G4)

8/191-1/12

$5 / 9-2 / 1$

8/191-2/17

$5 / 7-3 / 1$

$5 / 9-2 / 2$

Büdingen G-32 (I10D2)

$8 / 189-3 / 6$

$5 / 8-2 / 5$

$8 / 191-2 / 1$

$5 / 7-2 / 5$

Darmstadt F-12 (F1B2)

8/191-2/4

$5 / 7-3 / 2$

$5 / 7-2 / 1$

$5 / 7-2 / 5$

$5 / 8-1 / 7$

Dieburg G-33 (H1B2) 8/191-1/14

$5 / 8-1 / 3$

$5 / 8-2 / 6$

Dillenburg H-77 (H3D2) 8/191-1/12

$8 / 191-2 / 16$

$5 / 9-2 / 2$

Erbach H-60 (I8B2)

8/191-2/2

5/9-1/4

Eschwege $\mathrm{H}-65$ (I8C2) 5/9-1/8

5/9-1/15

Flörsheim I-147 (I13D2)

$$
\text { 8/191-1/9 }
$$

8/191-2/13

$5 / 9-3 / 13$

Frankenberg H-66 (I3C2)

$$
5 / 9-1 / 2,9
$$

5/9-1/9 
Friedberg G-34 (I11D2) 8/191-1/13 $5 / 8-2 / 7$

Fritzlar G-38 (14C2) 5/8-3/3 Fulda G-40 (H1D2)
8/195-1/8 $5 / 8-3 / 4$

$5 / 8-3 / 5$

Gelnhausen H-78 (I1D2) 8/191-1/6 $8 / 191-2 / 20$ $5 / 9-2 / 3$

GieBen G-35 (H5D2) 8/191-1/10 8/195-1/11

$8 / 191-2 / 7$

$5 / 7-3 / 3$

$8 / 195-1 / 10$

$5 / 8-3 / 1$

GroB-Gerau G-36 (H3B2) 8/191-1/3 $5 / 7-2 / 1$

$8 / 191-2 / 4$

$5 / 8-3 / 2$

Grunberg H-61 (H5D2) 5/9-1/5

Hadamar I-146 (I4D2) 8/191-2/22 $5 / 9-3 / 12$

Hanau H-79 (H2D2)

8/191-2/21

$5 / 9-2 / 4$

Hersfeld $\mathrm{H}-67$ (I1C2) 5/9-1/8

$5 / 9-1 / 10$

Hofgeismar H-68 (I5C2)

5/9-1/11

Hofheim H-81 (I8D2) 8/191-1/9

$8 / 191-2 / 13$

$5 / 9-3 / 1$

Hünfeld H-69 (H5C2) 5/9-1/12

Idstein/Bad Schwalbach H-85 (H6D2) 8/191-1/7 $8 / 191-2 / 18$ $5 / 9-3 / 5$

Kassel F-14 (H1C2) 5/8-2/2

Korbach G-48 (I6C2) $5 / 9-1 / 2$

Lauterbach H-62 (19C2) 8/191-2/3 $8 / 191-2 / 6$

$5 / 9-1 / 6$

Limburg/Weilburg H-80 (I6D2) 8/191-1/8 $8 / 191-2 / 22$ $5 / 9-2 / 4$

Marburg G-39 (F1C2) 5/9-2/6 
Melsungen $\mathrm{H}-70$ (I2C2)

5/8-2/2

$5 / 9-1 / 13$

Ober-Ramstadt H-64 (I4B2)

5/9-1/7

Offenbach F-13 (F2B2)

$8 / 189-3 / 5$

$8 / 191-1 / 5 \quad 5 / 8-2 / 1$

Rotenburg H-71 (I4A9)

5/9-1/8

$5 / 9-1 / 14$

Rüdesheim H-83 (I3G2)

8/191-1/7

$8 / 191-2 / 12 \quad 5 / 9-3 / 3$

Schlüchtern H-84 (I2G2)

5/9-3/4

$5 / 9-2 / 3$

$8 / 191-1 / 6 \quad 8 / 191-2 / 11$

Usingen I-149 (I12D2)

8/191-1/11

$5 / 9-3 / 15$

Weilburg H-82 (I7D2)

$5 / 9-3 / 2$

8/191-2/9

$8 / 191-1 / 2 \quad 8 / 191-1 / 8$

Wetzlar G-41 (I5D2)

8/191-1/4

$8 / 191-2 / 8 \quad 5 / 9-1 / 1$

Wiesbaden F-15 (F1D2)

8/191-1/2

$8 / 191-2 / 5$

$5 / 8-2 / 3$

Witzenhausen $\mathrm{H}-73$ ( $\mathrm{H} 2 \mathrm{C} 2)$

5/9-1/8

$5 / 9-1 / 15$

Wolfhagen I-144 (I7C2)

$5 / 9-3 / 10$

Ziegenhain H-74 (E2C2)

$5 / 9-1 / 16$

Inhaltsverzeichnis des Repertoriums zum Teilbestand Hessen (OMGHE)

der Akten der amerikanischen Militärregierung in Deutschland (OMGUS)

(Hessisches Hauptstaatsarchiv, Wiesbaden)

Band I 1. Detachments (Vorakten)

2. Residence Office

a) Wiesbaden

b) Frankfurt/M.

c) Darmstadt

Band II d) Marburg

e) Bad Hersfeld

f) Kassel

Band III 3. Personnel and Administration Division

Band IV 4. Historical Division 
Band V 5. Office of the Land Commissioner - Director (OLCH)

6. STEG (German Public Corporation for the Collection and Distribution of War Materials)

Band VI 7. Education and Religious Affairs Division/Education and Cultural Relations Division

Band VII

a) Office of the Chief

Band VIII

b) Education Branch

c) Higher Education Branch

d) University Officer

Band IX

e) Cultural Exchange Branch

f) Religious Affairs Branch

g) Theatre and Music Branch

h) Youth Activities Section

i) Adult Education Branch

Band $X$ 8. Civil Administration Division/Civil Affairs Division

a) Office of the Chief

b) Governmental Affairs Branch

c) Public Safety Branch

Band XI d) Public Health Branch

Band XII e) Public Welfare Branch

Band XIII f) Denazification Branch

Band XIV 9. Information Service Division

a) Office of the Chief

Band XV b) Radio Branch

c) Press Branch

d) Publication Branch

10. Public Relations Division

Band XVI 11. Legal Division

a) Prisons Branch

Band XVII

b) Legislation and Legal Counsel

Band XVIII 12. Intelligence Division

Band XIX 13. Economics Division
a) Office of the Director

Band XX b) Transportation Branch

c) Manpower Branch

d) Public Utilities Branch

Band XXI e) Industry Branch

Band XXII f) Food and Agriculture Branch

g) Decartelization Branch

Band XXIII 14. Bipartite Liaison Division

a) Economics Branch 
Band XXIV b) Finance Branch

Band XXV c) Economics Division / Finance Branch

Band XXVI 15. Property Division

a) Office of the Director

b) Reparation and Restitution Branch

Anhang:

Band XXVII 1. OMGUS Control Office Historical Division

2. USFET

Repertorium mit kommentierenden Stichworten

(Als Fundstelle wird nur das Shipment und die Nummer der Box angegeben.)

1. Detachments (Vorakten)

8/18-1 bis 8/19-1, 8/26-1 bis 8/27-1

Nur einzelne Folder der Detachments E-2, E-3, E-4, E-5 Wiedereröffnung der Grundschulen, Entnazifizierung der Lehrer, Universität Gießen (Verfilmungsquote minimal).

\section{Residence Office}

Allgemeine Korrespondenz, Kommunalverwaltung, Reports, öffentliches Leben, politische Betätigung, Gesundheit, Wohlfahrt, Displaced Persons, Entnazifizierung, Parteien, Beschlagnahmen, Gerichte der Militärregierung, Kriminalstatistik, Fluchtlinge, Unfallmeldungen, Internierungslager.

a) Wiesbaden $8 / 1-1$ bis $8 / 1-2$

b) Frankfurt/M. 8/1-2 bis $8 / 2-3$

Friedberg 8/3-1

Hanau 8/3-1

Bad Homburg $8 / 9-2$ bis $8 / 9-3,17 / 16-1$ bis $17 / 16-3$

c) Darmstadt 8/3-1, 8/11-3

Groß-Gerau 8/3-1, 8/3-2

Bergstraße 8/3-2

Dieburg $8 / 3-2$ bis $8 / 4-1$

Offenbach 8/4-1 bis 8/4-2

d) Marburg 8/5-1, 8/6-2, 8/12-1 bis 8/12-2

Dill 8/4-2

Biedenkopf 8/4-3, 8/6-2

Gießen 8/5-1 bis 8/5-3

e) Bad Hersfeld 8/5-2 bis 8/7-2

Fulda $8 / 7-2$ bis $8 / 8-1$

Ziegenhain 8/8-1, 8/8-2

Melsungen, Lauterbach, Rotenburg, Hünfeld 8/8-2

Alsfeld 8/8-2 bis 8/9-2

f) Kassel 8/9-3 bis 8/10-1, 8/11-2

Fritzlar 8/10-1 bis 8/11-1

Frankenberg, Hofgeismar/Wolfhagen 8/11-1

Waldeck 8/11-1, 8/11-3

Witzenhausen/Eschwege 8/11-2 


\section{Personnel and Administration Division}

8/178-1 bis $8 / 180-3,8 / 184-1,8 / 185-1$ bis $8 / 186-3$

Allgemeine Korrespondenz, US-Angestellte, Deutsche Angestellte, Bulletins, Special Orders, General Orders, Internierungslager, Reports, Information Control, Wochen- und Monatsberichte, Meinungsforschung.

\section{Historical Division}

\section{$8 / 187-1$ bis $8 / 191-2$}

Tagesjournale; wöchentliche, halbmonatliche und monatliche Berichte der Detachments, Divisions, einzelne Branches; Reports zu Entnazifizierung, Reparationen; Rohmaterial für die Zusammenstellung der Historical Reports von OMGHE.

5. Office of the Land Commissioner - Director

8/213-1 bis $8 / 217-3,17 / 15-3$

Allgemeine Korrespondenz, Flüchtlinge und DPs, Untersuchungen und Inspektionen, Anweisungen an deutsche Behörden, Wahlen, Freizeiteinrichtungen, Höhepunkte der Politik der Militärregierung, Politische Parteien, Special Orders, Reports, Korrespondenz mit Divisions, Branches, Detachments.

6. STEG (German Public Corporation for the Collection and Distribution of War Materials) (Staatliche Erfassungsgesellschaft für öffentliches Gut mbH) 17/163-1

7. Education and Religious Affairs Division / Education and Cultural Relations Division $8 / 18-1$ bis $8 / 24-3,8 / 26-1$ bis $8 / 29-3$

Allgemeine Korrespondenz, Reports, Schulwesen, Universitäten, Studenten, Kultusministerium, Entnazifizierung, Lehrpersonal, Berufsschulen, Fachschulen, Privatschulen, Kirchen, Sekten, Jugendorganisationen, Sportvereine, Expertenaustausch, Konferenzprotokolle, Jugendaktivitäten, Camps.

a) Office of the Chief

8/15-1 bis $8 / 17-3$

Korrespondenz, Expertenaustausch, Kultusministerium, Universitäten, Schulreform, Schulbücher, Erwachsenenbildung, Reorientation, Büchereien, Konferenzen, Kulturaustausch.

b) Education Branch

$8 / 18-1$ bis $8 / 22-3,8 / 24-1$ bis $8 / 24-3,8 / 44-2$ bis $8 / 45-2$

Korrespondenz, Schulbücher, Curricula, Entnazifizierung, Lehrpersonal, berufliche Erziehung, Lehrerausbildung, Statistik, Demokratisierung, Schulzeitungen, Reports, Schulreform, Studentenstatistik, Konferenzen, Tagungen.

c) Higher Education Branch $8 / 20-2$ bis $8 / 22-2$

Korrespondenz, Kultusministerium, Hessische Universitäten, Studenten, Austauschprogramme, Uni-Personal, Entnazifizierung, Veranstaltungen, Studentische Organisationen, Wohnungsprobleme, Forschung, Reports.

d) University Officer

8/18-3, 8/19-2, 8/24-3

Nur Universitäten Marburg und Gießen.

e) Cultural Exchange Branch

$8 / 19-2$ bis $8 / 20-2,8 / 24-3$

Kultur- und Expertenaustausch, Studentenaustausch. 
f) Religious Affairs Branch

8/26-2 bis $8 / 28-1$

Kirchen, Sekten, Entnazifizierung, Reports, Theologie, Studenten-Statistik.

g) Theater and Music Branch 8/24-3, 8/44-1

Report, Theater, Korrespondenz.

h) Youth Activities Section $8 / 28-3$ bis $8 / 32-3$

Korrespondenz, Jugendorganisationen, Jugendringe, Kreisjugendpfleger, LandesjugendausschuB, Sport, Camps, Jugendpublikationen, Jugendleiter, Reports über Einstellung von Jugendlichen, Konferenzen.

i) Adult Ecucation Branch

8/22-3

Korrespondenz, Reports, Konferenzen, Erwachsenenbildung, Volkshochschulen, Lehrpersonal, Programme, Austausch.

8. Civil Administration Division/Civil Affairs Division

a) Office of the Chief 8/45-2 bis 8/47-2, 8/49-2, 8/49-3

Korrespondenz mit deutschen Verwaltungen, Reports, Wahlen, Parteien, Amtsblätter, Kreisblätter, Gesetzestexte, Sitzungsprotokolle etc. (kaum verfilmt, da meist Doppeluberlieferung).

b) Governmental Affairs Branch

8/47-3 bis $8 / 49-1$

Öffentlicher Dienst; Parteien, Programme, Aktivitäten, Mitglieder, Interessenverbände.

c) Public Safety Branch

$8 / 50-1$ bis $8 / 50-3,8 / 53-1,8 / 53-2$

Allgemeine Korrespondenz, Anordnungen, Inspektionen, Polizei, Feuerwehr, Straftaten von Displaced Persons.

d) Public Health Branch

8/53-2 bis 8/63-1

Allgemeine Korrespondenz, Entnazifizierung des medizinischen Personals, Krankenhäuser, Gesundheitswesen, DP-Camps, Erhebungen über Krankheiten, Reports, Medizinisches Gerät und Arzneimittelversorgung.

e) Public Welfare Branch

8/63-1 bis $8 / 69-3$

Allgemeine Korrespondenz, Reports, Wohnungsprobleme, Jugendwohlfahrt, Displaced Persons, illegale Grenzübertritte, Verfolgte, Zulassung privater Wohlfahrtsorganisationen, Behinderte, Flüchtlingslager, Massenspeisungen.

f) Denazification Branch 8/78-1 bis $8 / 79-3$

Allgemeine Korrespondenz, Ministerium für politische Befreiung, Reports der Detachments, Berichte über Einzelpersonen, Spruchkammern, Internierungslager Darmstadt, Arbeitslager.

\section{Information Service Division}

a) Office of the Chief

8/153-2 bis 8/157-3

Allgemeine Korrespondenz, Reports, Kammern, Verbände, Vereine, Lizenzen, Lizenzträger, Presse, Verlage, Verleger, Pressegesetz, US- und deutsches Per- 
sonal, Regelungen, Konferenzen, Untersuchungsberichte, öffentliche Meinung.

b) Radio Branch

8/157-3, 8/161-2, 8/161-3, 8/172-1

Reports, Reaktionen der deutschen Bevölkerung auf Film, Radio, Zeitungen, Radio Frankfurt, Reden und Interviews.

c) Press Branch

$8 / 158-1$ bis $8 / 161-2,8 / 171-1$

Allgemeine Korrespondenz, Zeitungen (z.B. Frankfurter Rundschau, Hessische Nachrichten, Neuer Mainzer Anzeiger, Wiesbadener Kurier, Rheingauer Anzeiger, Frankfurter Neue Presse, Darmstädter Echo, Darmstädter Tagblatt), Personal, Lizenzträger, Lizenzen, Pressestatistik, DANA, Jugendzeitschriften, Entnazifizierung, Druckereien, Papierzuteilung, Buchhändler, Büchereien, Verleger, Reports.

d) Publication Branch

8/171-2 bis 8/172-2, 8/176-1, 8/176-2

Allgemeine Korrespondenz, Verleger, DP-Publikationen, Reports, NS-Literatur, Verbotene Literatur, Druckrechte.

10. Public Relations Division

8/191-3 bis 8/192-3

Allgemeine Korrespondenz, Erwachsenenbildung, Jugendarbeit, Frauenarbeit, Reorientation, Gemeinwesenarbeit, Expertenaustausch.

\section{Legal Division}

a) Prisons Branch

$8 / 196-1$ bis $8 / 212-3$

Gerichtsakten der einzelnen Gerichtsorte, Gnadengesuche, Einzelfălle, Reports (Verfilmungsquote 5-10\%).

b) Legislation and Legal Counsel

$17 / 202-1$ bis $17 / 212-3$

Korrespondenz, Justizministerium, Gerichte, Gesetzestexte, Ausführungsbestimmungen, Verordnungen, Spezielle Rechtsfragen, Reports, Organisationsfragen (Verfilmungsquote minimal).

12. Intelligence Division

8/193-2 bis $8 / 195-3,17 / 125-1$

Allgemeine Korrespondenz, Anordnungen, Reports, Politische Aktivitäten allgemein, Parteien, Gewerkschaften, einzelne Untersuchungsberichte uber Personen und Parteien.

\section{Economics Division}

a) Office of the Director

$8 / 80-1$ bis $8 / 86-3,17 / 19-1,17 / 19-2$

Allgemeine Korrespondenz, Wirtschaftsministerium, Entnazifizierung, Deutsche Kontrollämter, Interzonen- und Außenhandel, Kompensationsgeschäfte, Kohleversorgung, Preiskontrolle, Wirtschaftsstatistik, Wirtschaftsentwicklung, Produktion, Berichte über Firmen und Branchen, Hilfsaktionen.

b) Transportation Branch

$8 / 87-1$ bis $8 / 88-3,17 / 19-2$

Aligemeine Korrespondenz, Wirtschaftsministerium, Verkehrswege, Entnazifizierung, Transportprobleme, Energieversorgung, Reports, Kraftfahrzeuge. 
c) Manpower Branch

8/89-1, 8/89-2, 8/115-2, 8/115-3, 8/118-1

Allgemeine Korrespondenz, Ministerium für Arbeit und Wohlfahrt, Arbeitskräfteprobleme, Gewerkschaften, Arbeitsrecht, Betriebsräte, Entnazifizierung, Arbeitsverwaltung, Sozialversicherung.

d) Public Utilities Branch

8/91-2, 8/91-3, 17/19-1

Allgemeine Korrespondenz, Wirtschaftsministerium, Straßenbauverwaltung, Reports, Zustandsberichte Verkehrswege, öffentliche Energieversorgung.

e) Industry Branch

8/91-3 bis 8/94-3, 17/25-1, 17/25-2

Allgemeine Korrespondenz, Reports, einzelne Firmen, Zuteilung von Rohund Werkstoffen, Energieversorgung; Produktionsberichte über Firmen, Industriezweige, Produkte; Lieferverträge; Bestandsaufnahmen über Betriebe, die die militärische Sicherheit gefährden.

f) Food and Agriculture Branch

$8 / 118-1$ bis $8 / 126-3,17 / 15-1,17 / 15-3,17 / 24-2,17 / 163-2,17 / 163-3$

Allgemeine Korrespondenz, Landwirtschaftsministerium, Reports, Nahrungsmittelproduktion und Produkte, Feldpolizei, Forstverwaltung, Kammern, Berufsverbände, Lebensmitteldiebstăhle, Landesemährungsamt, Nahrungsmittelmangel, Landwirtschaftsstatistik, Düngemittel, Jagd und Fischerei, Expertenaustausch, Entnazifizierung, Landreform, Nahrungsmittelstatistik.

g) Decartelization Branch

$17 / 8283$ bis $17 / 8290$

Allgemeine Korrespondenz, Reports und Erhebungen zur Entflechtung einzelner Firmen (z.B. Bosch GmbH, Henschel und Sohn GmbH, DEGUSSA, E. Merck, Spinnfaser AG, Wintershall AG).

14. Bipartite Liaison Division

a) Economics Branch

$17 / 19-2,17 / 164-1$ bis $17 / 166-3,17 / 169-1$

Allgemeine Korrespondenz, Reports, Bestandsaufnahmen von Firmen in der US-Zone, einzelne Produkte, Untersuchung und Befragung IG-Farben, chemische und angeschlossene Industrie, Energieversorgung, Export - Import, Reparationen, Kriegsindustrie, Entflechtung, Währungsreform, Außenhandelsbeziehungen.

b) Finance Branch

$17 / 169-2$ bis $17 / 170-2$

Marshallplan, Kreditpolitik, Devisen, ausländische Gutachten, Ansprüche von Kriegsgefangenen, gespertte Konten, Landeszentralbank, Preise, Zollverwaltung, Zölle, Zollunion, Konferenzen.

c) Economics Division / Finance Branch

$8 / 103-1$ bis $8 / 105-3,8 / 113-1$ bis $8 / 114-3,8 / 176-2,17 / 19-3$ bis $17 / 24-1$

Allgemeine Korrespondenz, Finanzministerium, Landeszentralbank, Reports, Banken, Finanzberichte, Pensionen, Beutegut, gesperrte Konten, Zollämter, Staatshaushalt, Steuern, Besatzungskosten, Lastenausgleich, Währungsreform, Entnazifizierung, Finanzämter.

15. Property Division

a) Office of the Director

$8 / 128-1$ bis $8 / 137-2$ 
Allgemeine Korrespondenz, Vermögenskontrolle, Treuhänder, ehemalige Besitzer zwangsenteigneter Güter, individuelle und institutionelle Ansprüche auf Rückerstattung beschlagnahmter Vermögenswerte, Wehrmachtseigentum, Entflechtung, Reports.

b) Reparation and Restitution Branch 8/137-2 bis $8 / 151-3$

Reparationsgüter, Inventarisierung, einzelne Firmen, Zerstörung der Kriegsindustrie, Ruckerstattung, Wiedergutmachung, Munitionsverwertungsprogramme, Reports, Landesamt für Vermögenskontrolle und Wiedergutmachung, AbschluBbericht.

\section{OMGUS Control Office Historical Division}

$3 / 437-1,5 / 7-2$ bis $5 / 9-3,5 / 41-1$ bis 5/43-1, 5/53-1, 11/17--3

Historical Reports OMGHE; verschiedenartigste Reports der Detachments, Divisions, Branches; statistisches Material und statistisches Handbuch der Economics Division.

\section{USFET}

USFET-Akten (RG 338) Box Nr. 11063 - 11065, 11068

After Action Reports, Truppenbewegungen

The Adjutant General's Office (RG 407) Box Nr. 2673

After Action Report Nr. 155 (G-5, 7 th Army)

Detachment-Standorte Südhessen.

Wie sich aus den Akten der Divisions immer wieder Hinweise auf lokale und regionale Entwicklungen ergeben, findet man in den Akten der Detachments und Liaison \& Security Offices Material zu übergreifenden Fragestellungen. Deshalb wird jede Arbeit mit den Akten der amerikanischen Militärregierung in Hessen bis auf weiteres mit einer systematischen Durchsicht des gesamten Repertoriums beginnen müssen. Unsere knappe Skizze der organisatorischen und funktionalen Gliederung von OMGHE sowie unsere Bemerkungen zum Quellenbestand sollten dabei, so hoffen wir, orientierende Hilfestellungen geben können.

\section{Deutsche Quellen zur amerikanischen Militärregierung in Hessen}

Wer über die amerikanische Militärregierung in Hessen arbeiten möchte, muß zum beschriebenen amerikanischen Quellenmaterial die Bestände der deutschen Archive heranziehen. In Stadt- und Kreisarchiven finden sich Unterlagen zu den lokalen Gliederungen der Militärregierungen, die in der Korrespondenz zwischen Kreisen und Gemeinden auf der einen und der Militärregierung auf der anderen Seite angefallen sind. Da diese Quellen in von Ort zu Ort sehr unterschiedlichem Maß erhalten und verzeichnet sind, können darüber keine allgemeinen Aussagen gemacht werden. Gleiches gilt für die ersten deutschen Regierungen in Hessen, deren Akten teilweise in den hessischen Staatsarchiven zugänglich sind. Für die Provinzialregierung Starkenburg und für den Regierungsbezirk Darmstadt ist das Staatsarchiv Darmstadt zuständig, während die Materialien für Hessen-Nassau vom Staatsarchiv Marburg verwaltet werden. Im Hauptstaatsarchiv Wiesbaden befinden sich die Quellen zum Regierungsbezirk Wiesbaden.

Im Hauptstaatsarchiv sind auch die wichtigsten deutschen Quellen zu OMGHE in Wiesbaden zugänglich. Sie sind aus der Zusammenarbeit zwischen OMGHE und der 
hessischen Landesregierung hervorgegangen und befinden sich in folgenden Abteilungen des Hauptstaatsarchivs:

Abt. 501 Der Hessische Minister für politische Befreiung (bis 1946 Minister für politische Bereinigung, bis 1947 Minister für Wiederaufbau und politische Befreiung)

Abt. 502 Der Hessische Ministerpräsident - Staatskanzlei (bis 1947 Ministerpräsident - Chef der Staatskanzlei)

Ábt. 503 Der Hessische Minister des Innern (bis 1947 Minister des Innern)

Abt. 504 Der Hessische Kultusminister (bis 1947 Minister für Kultur und Unterricht; 1947-1949 Hessischer Minister für Kultus und Unterricht)

Abt. 505 Der Hessische Minister der Justiz (bis 1947 Minister der Justiz)

Abt. 506 Der Hessische Minister der Finanzen (bis 1947 Minister der Finanzen)

Abt. 507 Der Hessische Minister für Wirtschaft und Technik (bis 1947 Minister für Wirtschaft und Verkehr)

Abt. 508 Der Hessische Sozialminister (bis 1947 Minister für Arbeit und Volkswohlfahrt)

Abt. 509 Der Hessische Minister für Landwirtschaft und Forsten (1947-1950 Hessischer Minister für Landwirtschaft, Ernährung und Forsten)

Da der Ministerpräsident gegenüber OMGHE die Gesamtverantwortung für die Politik der Landesregierung trug, ist die Abteilung 502 besonders bedeutsam. Hier ist ein umfangreicher Schriftwechsel mit der Militärregierung vorhanden (Behördenregistraturzeichen 2e). In der gleichen Abteilung finden sich auch Unterlagen zur Wohnungsbeschlagnahmung durch die Militärregierung. Allerdings sind auch im Geschäftsgang der anderen Ministerien hin und wieder Akten im Umgang mit der Militärregierung angefallen. Hervorzuheben sind das Ministerium für politische Befreiung, das Ministerium des Innern und das Wirtschaftsministerium, in dem auch Akten zur Auseinandersetzung um die Sozialisierung angefallen sind.

In den Nachlässen der ersten Ministerpräsidenten Karl Geiler (Hauptstaatsarchiv, Abt. 1126), seines Nachfolgers Christian Stock (Staatsarchiv Darmstadt, Bestand 0-27), des Erziehungsministers Erwin Stein (Hauptstaatsarchiv Wiesbaden, Abt. 1178), des zeitweiligen Chefs der Staatskanzlei Hermann Brill (Bundesarchiv, Koblenz) und des Chefs der Provinzialregierung Starkenburg Ludwig Bergsträsser (Institut für Wissenschaftliche Politik, Universität Marburg) finden sich weitere Quellen zur Militärregierung 
